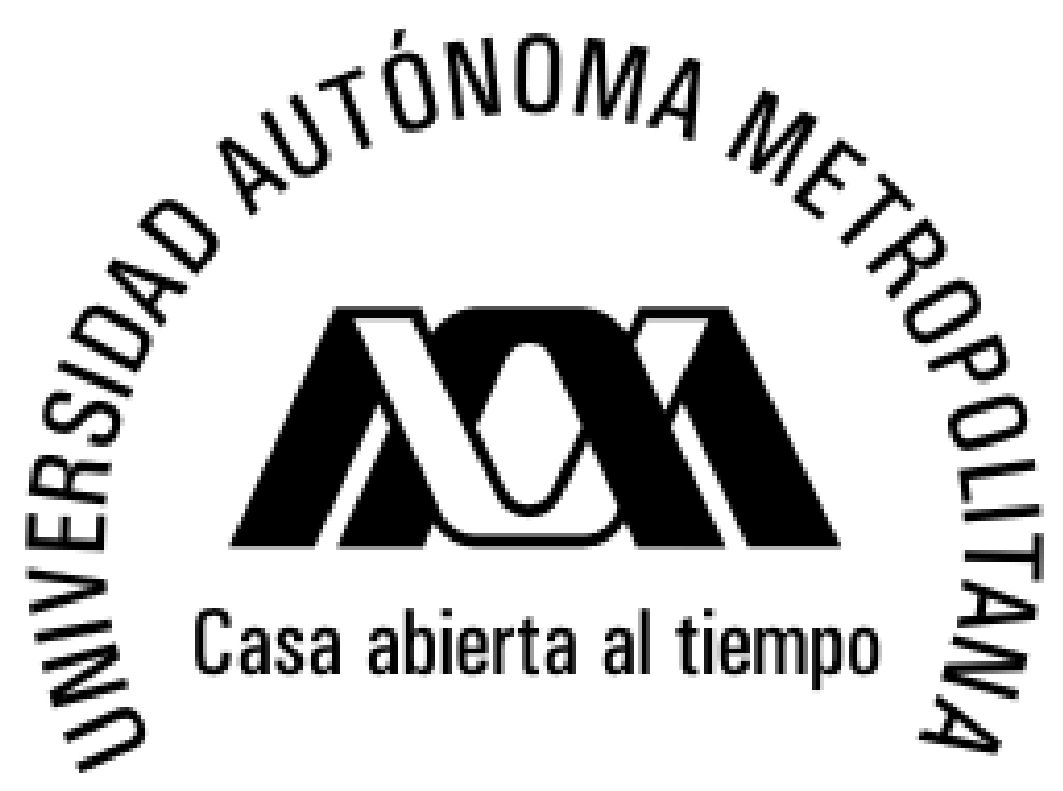

“Mi cuerpo es de este mundo, pero mi espíritu es de DIOS"

Esta tesis está dedicada a dos grandes maestros que DIOS me concedió en la vida: $A$ mi madre Rosa Cristina y a mi hermano Juan José. 


\section{REDUCCIÓN ADAPTABLE DE LA INTERFERENCIA ACÚSTICA CARDIACA EN SONIDOS RESPIRATORIOS}

Tesis que se presenta para obtener el grado de

Maestría en Ciencias (Ingeniería Biomédica).

Luis Felipe Domínguez Robert

Asesores:

Dra. Sonia Charleston Villalobos

Universidad Autónoma Metropolitana
Dr. Ramón González Camarena

Universidad Autónoma Metropolitana

\section{Sinodales:}

Dra. María Teresa García González Universidad Autónoma Metropolitana
Dra. Sonia Charleston Villalobos Universidad Autónoma Metropolitana

Dra. Georgina Chi Lem

Instituto Nacional de Enfermedades

Respiratorias "Ismael Cosio Villegas"

UNIVERSIDAD AUTÓNOMA METROPOLITANA - IZTAPALAPA

DIVISIÓN DE CIENCIAS BÁSICAS E INGENIERÍA

POSGRADO EN INGENIERÍA BIOMÉDICA

MÉXICO D.F.

AGOSTO 2008 


\section{AGRADECIMIENTOS}

Agradezco el gran apoyo otorgado por el Departamento de Ingeniería Biomédica del Instituto Nacional de Enfermedades Respiratorias encabezado por:

M. en I. Ricardo Noé Rodríguez Vera

Jefe del Departamento de Ingeniería Biomédica

M. en I. Ana Bertha Pimentel Aguilar

Coordinadora del Departamento de Ingeniería Biomédica

A mis compañeros de trabajo:

Ing. Adrián Pacheco López.

Ing. Jehú Martínez Pablo.

Ing. Josefina García Dávila.

Ing. Arturo Martínez Rivera.

Ing. Gianina Velázquez Duarte.

Un agradecimiento especial a la Ing. Karla Velázquez Pérez, por su valiosa ayuda en la investigación, y a la Ing. María de Montserrat Godínez García por su gran ayuda desinteresada en la programación para el procesamiento de las señales expuestas en esta tesis.

A mis grandes compañeros Gabriel, Graciela, Martín, Ángel, Oscar, Marco y Sergio del Posgrado en Ingeniería Biomédica. 


\section{ÍNDICE GENERAL}

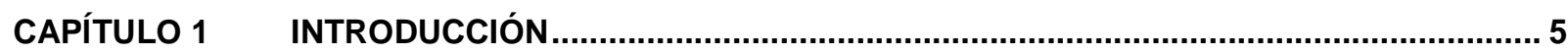

1.1 El Sistema Respiratorio, los Sonidos Asociados y su Importancia Clínica............................. 5

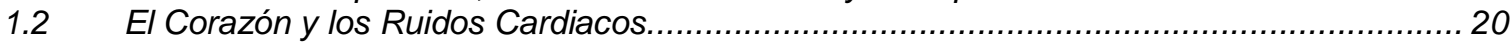

CAPÍTULO 2 PLANTEAMIENTO DEL PROBLEMA Y ANTECEDENTES...................................... 23

2.1 ¿Porqué los Ruidos Cardiacos Representan una Interferencia para el Análisis de los Sonidos Respiratorios? ..................................................................................... 23

2.2 Otros Intentos en la Reducción de la Interferencia Acústica Cardiaca................................. 27

CAPÍTULO 3 JUSTIFICACIÓN DE LA METODOLOGÍA PROPUESTA Y OBJETIVOS.................. 30

3.1 Justificación de la Metodología Propuesta..................................................................... 30

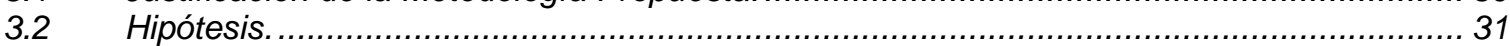

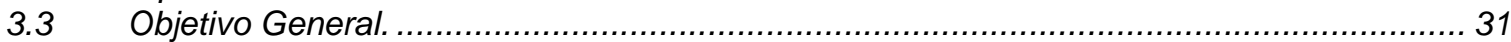

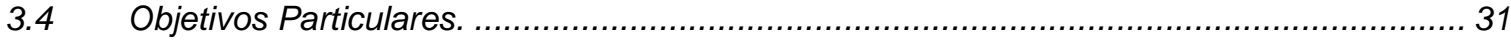

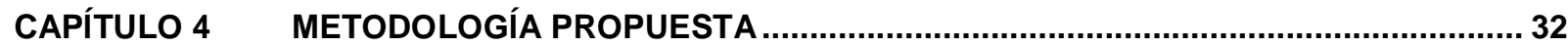

4.1 Esquema para la Cancelación de la Interferencia Acústica Cardiaca............................... 32

4.1.1 Descomposición de la Señal de Interés en Modos de Oscilación. ................................................33

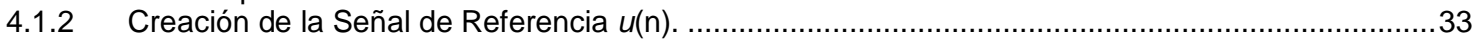

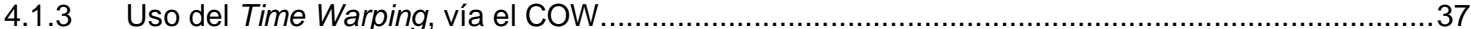

4.1.4 Esquema de Eliminación de Interferencias por Filtrado Adaptativo. ..............................................38

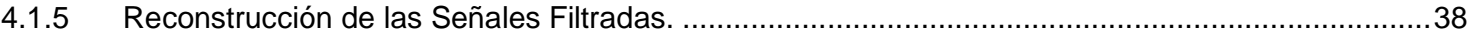

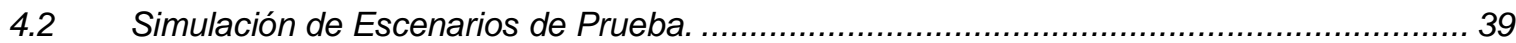

4.3 Adquisición Multicanal de Información Acústica en Sujetos Sanos................................... 42

4.4 Medidas de Desempeño del Esquema Propuesto........................................................ 44

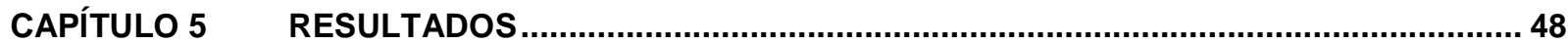

5.1 Información Acústica Presente en las Señales Multicanal Adquiridas. ................................ 48

5.2 Modos de Oscilación (IMFs) en Señales Simuladas y Adquiridas Reales........................... 48

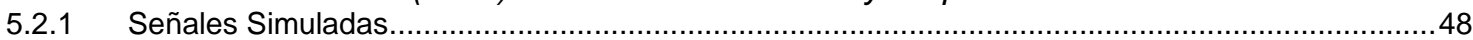

5.2.1.1 Señales Acústicas Simuladas con Diferente Nivel de Ruido de Fondo. ...................................48

5.2.1.2 IMFS de las Señales Simuladas...................................................................................49

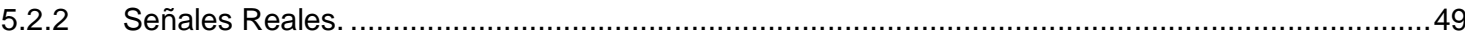

5.2.2.1 EMD de Señales Acústicas en Posiciones Cercanas al Corazón............................................49

5.2.2.2 EMD de Señales Acústicas en Posiciones Alejadas del Corazón. ………….........................50

5.2.2.3 Señales Simuladas Filtradas con TW y sin TW. ...............................................................50

5.2.2.4 Señales Reales Filtradas con TW y sin TW...…………………………………….......5

5.3 Medidas de Desempeño en Escenarios de Prueba........................................................ 51

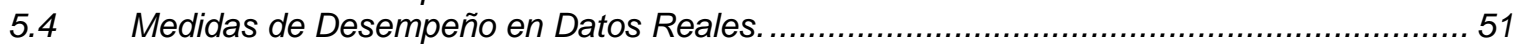

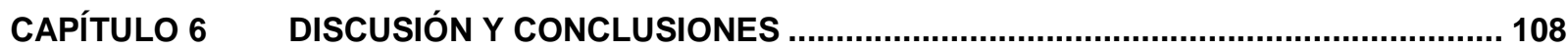

6.1 Análisis Cualitativo de la Morfología de las Señales Acústicas Torácicas Adquiridas. ...... 108

6.2 EMD en el Procesamiento de las Señales Acústicas Adquiridas: Análisis Cualitativo de los Modos de Oscilación. ................................................................................. 109

6.3 Análisis Cualitativo de las Señales Adquiridas vs. Filtradas por la Metodología Propuesta. 111

6.4 Desempeño de la Metodología Propuesta con Base en el Análisis de Potencias Espectrales. 112

6.5 Conclusiones.

A.1 FíSICA DEL SONIDO.

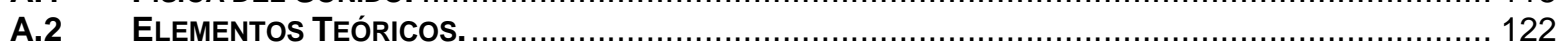

REFERENCIAS. 139 


\section{ÍNDICE DE FIGURAS}

Figura 1.1 Nomenclatura aplicada a las vías respiratorias y al parénquima pulmonar. Representación esquemática de las subdivisiones de las vías aéreas de conducción y de las unidades respiratorias terminales.

Figura 1.2 Sistema traqueobronquial normal. Representación esquemática del árbol traqueobronquial normal en la que se observa la numeración ordinaria de los bronquios segmentarios en una proyección posteroanterior.

Figura 1.3 Árbol traqueobronquial normal. La numeración se refiere a los segmentos normales de los pulmones derecho (D) e izquierdo (I), según la lista que

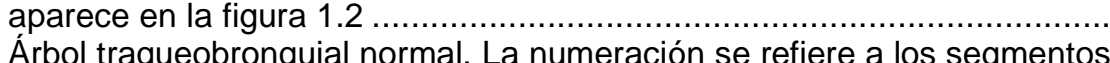

Figura 1.4 Árbol traqueobronquial normal. La numeración se refiere a los segmentos (OIA) y oblicua derecha anterior (ODA)

Figura 1.5 Esqueleto de tórax normal, en el que se observan costillas, esternón y columna torácica. La línea punteada indica la posición de la cúpula diafragmática durante la capacidad residual funcional.

Figura 1.6 Anatomía del corazón

IMFs 1 a 5 de la señal acústica adquirida cerca del corazón que corresponde a la zona de auscultación de la válvula mitral.

Figura 4.3 IMFs 6 a 10 de la señal acústica adquirida cerca del corazón que corresponde a la zona de auscultación de la válvula mitral.

Figura 4.4

Figura 4.5 IMFs 11 a 15 de la señal acústica adquirida cerca del corazón que corresponde a la zona de auscultación de la válvula mitral.

$\mathrm{IMF}_{6}$ de la Figura 4.2 con componentes cuasi-periódicos pertenecientes a ruidos cardiacos. El recuadro a la izquierda indica el segmento de apnea que se utilizará para generar la señal de referencia.

Figura 4.6

Modo de oscilación seleccionado con componentes cuasi-periódicos pertenecientes a ruidos cardiacos. La gráfica superior representa el $\operatorname{IMF}_{6}$ y la inferior su envolvente vía la transformada de Hilbert.

Figura 4.7

Se observa la misma envolvente que en la figura inferior 4.6 y con asteriscos se indican los máximos localizados de la función de correlación cruzada.

Figura 4.8

Figura 4.9

Figura 4.10 Figura 4.11

Figura 4.12

Figura 4.13

Figura 4.14 Figura 4.15

Figura 5.1 Figura 5.2 Figura 5.3

Figura 5.4
En color oscuro se muestra la señal de referencia y en color gris se muestra la señal que es deformada para mejorar la alineación. En la gráfica superior se observan ambas señales antes de aplicar el algoritmo COW mientras que

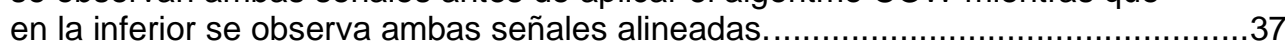

Esquema de eliminación de interferencias por filtrado adaptativo. ........................38

Morfología de los ruidos cardiacos involucrados en la señal simulada...................40

Señal de ruidos cardiacos en posiciones conocidas. Nótese el cambio de morfología de los primeros ruidos cardiacos a lo largo de la señal.....

Ventanas de Hamming para definir las fases de inspiración y espiración de la señal simulada.

Señal respiratoria simulada incluyendo ruidos cardiacos, ruido de fondo y sonido respiratorio con tres ciclos respiratorios completos y una etapa de apnea inicial.

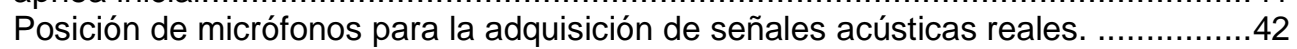

(Aquí se reproduce la Figura 2.1).Señales acústicas adquiridas. En la parte superior se muestra el flujo respiratorio (2L/seg), IZQ1 a IZQ3 corresponden a las señales de la línea axilar izquierda, DER1 a DER 3 corresponden a las señales de la línea derecha axilar, y las cinco últimas corresponden a puntos próximos al corazón

Señal acústica simulada con una relación señal-ruido de fondo de $20 \mathrm{~dB} \ldots \ldots \ldots \ldots \ldots . \ldots 3$

Señal acústica simulada con una relación señal-ruido de fondo de $50 \mathrm{~dB}$...............53

IMFs 1 al 5 de la señal acústica simulada con una relación señal-ruido de fondo de $20 \mathrm{~dB}$.

IMFs 6 al 10 de la señal acústica simulada con una relación señal-ruido de fondo de $20 \mathrm{~dB}$. 
Figura 5.5 IMFs 11 al 15 de la señal acústica simulada con una relación señal-ruido de fondo de $20 \mathrm{~dB}$.

Figura 5.6

Figura 5.7

IMFs 1 al 5 de la señal acústica simulada con una relación señal-ruido de

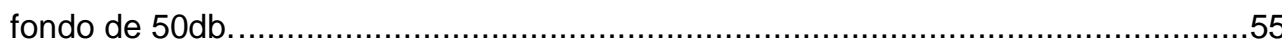

IMFs 6 al 10 de la señal acústica simulada con una relación señal-ruido de

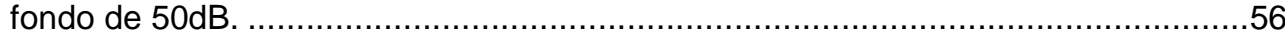

Figura 5.8 IMFs 11 al 15 de la señal acústica simulada con una relación señal-ruido de

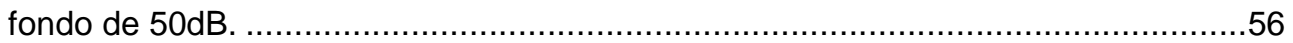

Figura 5.9

Figura 5.10

Señales acústicas obtenidas de las zonas de auscultación de las válvulas

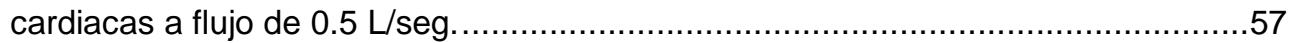

Figura 5.11

Señales acústicas de la línea media axilar izquierda a flujo de $0.5 \mathrm{~L} / \mathrm{seg} \ldots \ldots \ldots \ldots \ldots . . \ldots 7$

Figura 5.12

Figura 5.13

Señales acústicas de la línea media axilar derecha a flujo de $0.5 \mathrm{~L} / \mathrm{seg}$...............58

Señales acústicas obtenidas de las zonas de auscultación de las válvulas

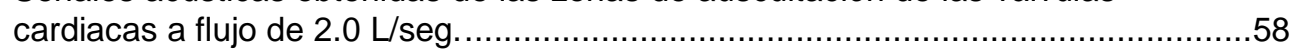

Figura 5.14

Señales acústicas de la línea media axilar izquierda a flujo $2.0 \mathrm{~L} / \mathrm{seg} . . . \ldots \ldots \ldots \ldots \ldots . . . . . .59$

Figura 5.15

Figura 5.16

Señales acústicas de la línea media axilar derecha a flujo de $2.0 \mathrm{~L} / \mathrm{seg} . . . . . . . . . . . . . .59$

IMFs 1 al 5 de la señal acústica obtenida de un sujeto sano en la zona de

auscultación que corresponde a la válvula tricúspide a flujo de $0.5 \mathrm{~L} / \mathrm{seg}$. .....

IMFs 6 al 10 de la señal acústica obtenida de un sujeto sano en la zona de

auscultación que corresponde a la válvula tricúspide a flujo de $0.5 \mathrm{~L} / \mathrm{seg}$................60

Figura 5.17 IMFs 11 al 15 de la señal acústica obtenida de un sujeto sano en la zona de auscultación que corresponde a la válvula tricúspide a flujo de $0.5 \mathrm{~L} / \mathrm{seg}$................61

Figura 5.18

IMFs 1 al 5 de la señal acústica obtenida de un sujeto sano en la línea axilar

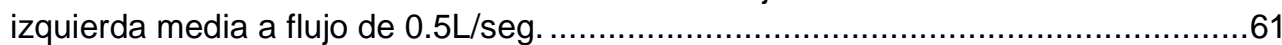

Figura 5.19 IMFs 6 al 10 de la señal acústica obtenida de un sujeto sano en la línea

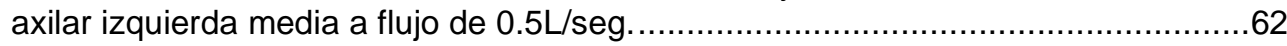

Figura 5.20 IMFs 11 al 15 de la señal acústica obtenida de un sujeto sano en la línea

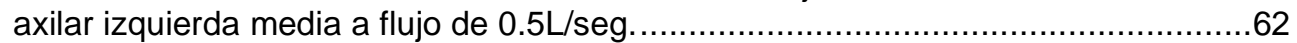

Figura 5.21

IMFs 1 al 5 de la señal acústica obtenida de un sujeto sano en la línea axilar derecha media a flujo de $0.5 \mathrm{~L} / \mathrm{seg}$.

IMFs 6 al 10 de la señal acústica obtenida de un sujeto sano en la línea

axilar derecha media a flujo de $0.5 \mathrm{~L} / \mathrm{seg}$.

Figura 5.22

Figura 5.23

IMFs 11 al 15 de la señal acústica obtenida de un sujeto sano en la línea

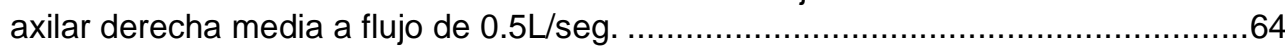

Figura 5.24 IMFs 1 al 5 de la señal acústica obtenida de un sujeto sano en la línea axilar

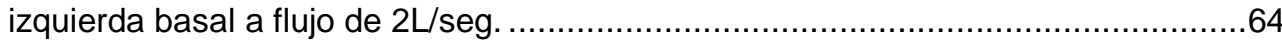

Figura 5.25 IMFs 6 al 10 de la señal acústica obtenida de un sujeto sano en la línea axilar izquierda basal a flujo de $2 \mathrm{~L} / \mathrm{seg}$.

Figura 5.26

IMF's 11 al 15 de la señal acústica obtenida de un sujeto sano en la línea

axilar izquierda basal a flujo de 2L/seg.

Figura 5.27 Señal simulada con una relación señal respiratoria-ruido cardiaco de $50 \mathrm{~dB}$ y

Figura 5.28

Figura 5.29

Señal simulada con una relación señal respiratoria-ruido cardiaco de $20 \mathrm{~dB}$ y

sus señales filtradas.

Señal obtenida de la línea axilar izquierda apical y sus señales filtradas a

Figura 5.30 Señal obtenida de la línea axilar izquierda media y sus señales filtradas a

flujo de $0.5 \mathrm{~L} / \mathrm{seg}$.

Figura 5.31

Señal obtenida de la línea axilar izquierda basal y sus señales filtradas a

flujo de $0.5 \mathrm{~L} / \mathrm{seg}$.

Figura 5.32

Figura 5.33 de $0.5 \mathrm{~L} / \mathrm{seg}$.

Señal obtenida de la línea axilar derecha media y sus señales filtradas a

flujo de $0.5 \mathrm{~L} / \mathrm{seg}$.

Figura 5.34

Señal obtenida de la línea axilar derecha basal y sus señales filtradas a flujo

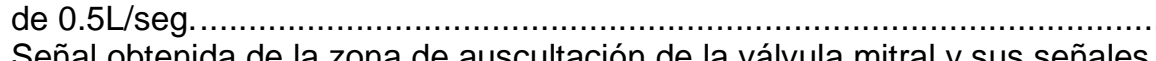

Figura 5.35

Señal obtenida de la zona de
filtradas a flujo de $0.5 \mathrm{~L} / \mathrm{seg}$. 
Figura 5.36 Señal obtenida de la zona de auscultación de la válvula tricúspide y sus señales filtradas a flujo de $0.5 \mathrm{~L} / \mathrm{seg}$.

Figura 5.37 Señal obtenida de la zona de auscultación de la válvula pulmonar y sus señales filtradas a flujo de $0.5 \mathrm{~L} / \mathrm{seg}$.

Figura 5.38

Señal obtenida de la zona de auscultación de la válvula aórtica y sus

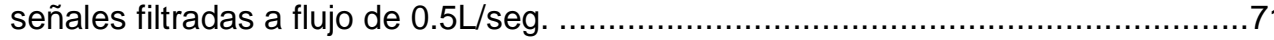

Figura 5.39 Señal obtenida de la zona de auscultación precordial promedio y sus

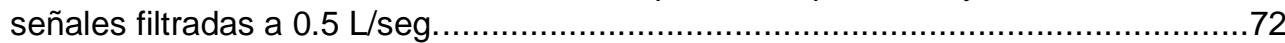

Figura 5.40 Señal obtenida de la línea axilar izquierda apical y sus señales filtradas a

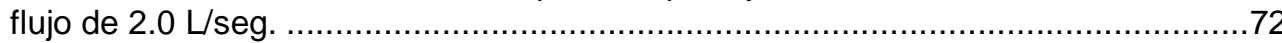

Figura 5.41 Señal obtenida de la línea axilar izquierda media y sus señales filtradas a

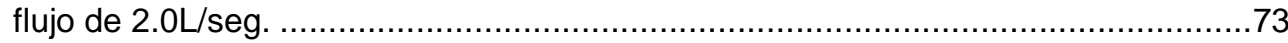

Figura 5.42 Señal obtenida de la línea axilar izquierda basal y sus señales filtradas a

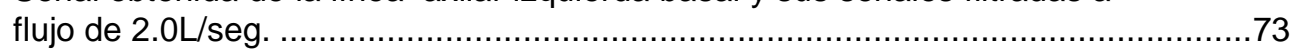

Figura 5.43 Señal obtenida de la línea axilar derecha apical y sus señales filtradas a flujo

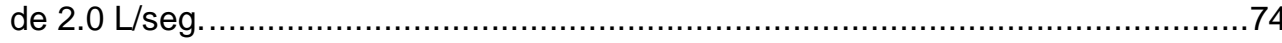

Figura 5.44 Señal obtenida de la línea axilar derecha media y sus señales filtradas a flujo

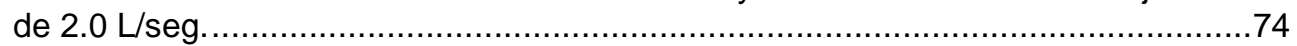

Figura 5.45 Señal obtenida de la línea axilar derecha basal y sus señales filtradas a flujo

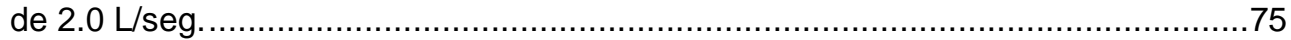

Figura 5.46 Señal obtenida de la zona de auscultación de la válvulas mitral y sus señales

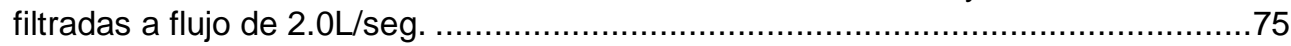

Figura 5.47 Señal obtenida de la zona de auscultación de la válvula pulmonar y sus

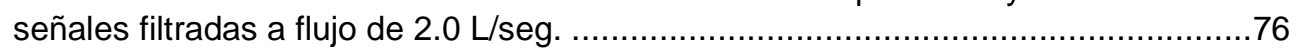

Figura 5.48 Señal obtenida de la zona de auscultación precordial promedio y sus

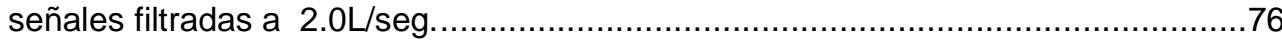
77

Figura 5.49 Representación tiempo-frecuencia de la señal acústica simulada con una relación señal- ruido de fondo de $20 \mathrm{~dB}$.

Figura 5.50

Figura 5.51 Representación tiempo-frecuencia de la señal acústica simulada con una relación señal-ruido de fondo de $50 \mathrm{~dB}$.

Potencias promedio de todos los sujetos a flujo de $0.5 \mathrm{~L} / \mathrm{seg}$ en diferentes bandas de frecuencia.

Figura 5.52 Potencias promedio de
bandas de frecuencia. 105

Figura 5.53 Potencias promedio de todos los sujetos a flujo de 1.5L/seg en diferentes bandas de frecuencia

Figura 5.54 Potencias promedio de todos los sujetos a flujo de $2.0 \mathrm{~L} / \mathrm{seg}$ en diferentes bandas de frecuencia. 


\section{CAPÍTULO 1 INTRODUCCIÓN}

\subsection{El Sistema Respiratorio, los Sonidos Asociados y su Importancia Clínica.}

Con respecto a la anatomía de las vías aéreas, se debe tener en cuenta que se dividen en superior e inferior, la vía aérea superior la integra: la nariz, boca, laringe, tráquea, en cambio, la vía aérea inferior está constituida por bronquios, bronquiolos, alvéolos y el tejido de sostén de ésta vía conocido como parénquima pulmonar; sin mencionar que la integridad de la caja torácica con la función de los músculos respiratorios primarios (diafragma, inspiratorios y espiratorios) permiten la ventilación es decir el movimiento de entrada y salida del aire [1]. La descripción completa de la vía respiratoria debe incluir los órganos respiratorios a nivel del sistema nervioso central como: el encéfalo, médula espinal, nervios periféricos, las vías nerviosas que inervan los músculos primarios y secundarios de la ventilación, sin embargo estos últimos elementos nerviosos no serán tratados en esta tesis.

Aunque las vías respiratorias intra y extra torácicas se distinguen anatómicamente, se trata de un sistema continuo que desde un punto de vista anatomofuncional, conviene subdividir en dos componentes (Figura 1.1): vías de conducción (generación 0 a 16) y unidades respiratorias terminales (generación 17 a 24). Estos sistemas se originan de una división dicotómica y asimétrica por cada generación de tal forma que terminan con los alvéolos cuya resistencia al pase de aire es mínima y la superficie de intercambio gaseoso es grande $\left(70 \mathrm{~m}^{2}\right.$ aproximadamente). Las vías de conducción sirven para llevar el aire hacia el interior y el exterior del tórax, y en las unidades respiratorias (alveólos) se lleva a cabo el intercambio gaseoso es decir entra el oxígeno y sale bióxido de carbono del torrente circulatorio. El proceso estricto de la respiración es a nivel mitocondrial.

\section{VÍAS RESPIRATORIAS DE CONDUCCIÓN.}

El aire viaja por las vías aéreas superiores: nariz, faringe, larínge, tráquea, donde es filtrado, humedecido, calentado y depositado en la vía aérea inferior: tráquea intra torácica, bronquios, bronquiolos, conductos alveolares, sacos alveolares. 


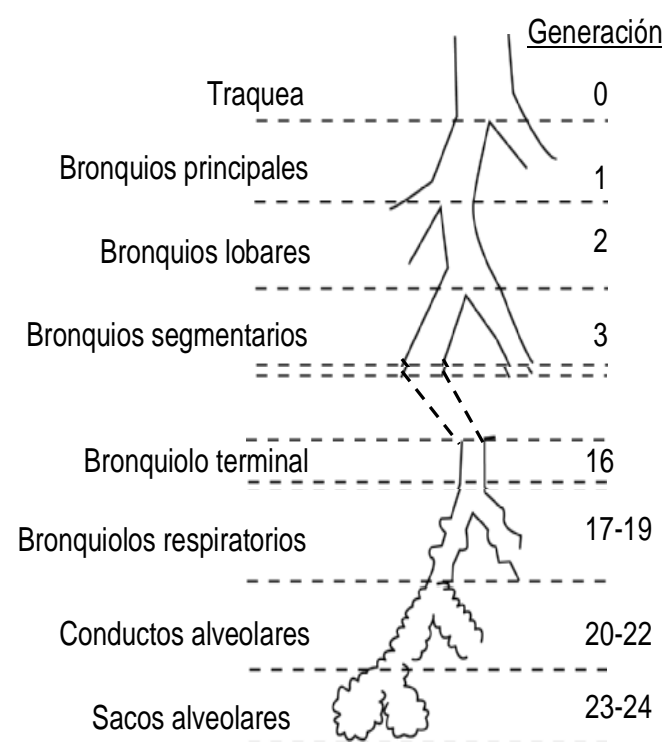

Figura 1.1 Nomenclatura aplicada a las vías respiratorias y al parénquima pulmonar. Representación esquemática de las subdivisiones de las vías aéreas de conducción y de las unidades respiratorias terminales.

\section{FILTRACIÓN.}

La superficie nasal tiene unos $60 \mathrm{~cm}^{2}$. El aire es filtrado a través de las vibrisas (vellos que se ubican en la entrada de las fosas anteriores o en el vestíbulo nasal) que debido a su movimiento antero posterior arrastran los cuerpos extraños hacia atrás con el moco que es deglutido y eliminado por el aparato digestivo.

\section{HUMIDIFICACIÓN.}

La humedad es indispensable para la adecuada actividad y preservación de los cilios. La nariz se encarga de proporcionar al aire inspirado una humedad relativa aproximadamente de $85 \%$; los cornetes participan en forma activa en este intercambio de calor y agua. Durante la inspiración se agrega humedad y en la espiración la humedad va de vuelta a la mucosa.

\section{Calentamiento del aire.}

Esta función se lleva a cabo a través de los plexos venosos, la malla de capilares y el tejido eréctil mucoso y submucoso tanto del tabique como de los cornetes nasales. Esto facilita el intercambio de oxígeno y bióxido de carbono en los pulmones. 


\section{Resistencia nasal.}

Nariz, faringe, tráquea, bronquios y pulmones son estructuras que ofrecen resistencia al paso del aire durante la inspiración y espiración. Las vías aéreas superiores causan 50 a $70 \%$ de la resistencia total al paso del aire, siendo más variable que la ofrecida por las vías aéreas inferiores. El flujo de aire en la nariz cuenta con dos tipos de presión, negativa durante la inspiración y positiva en la espiración, por lo que, al registrar las presiones durante el ciclo respiratorio y conociendo el flujo de aire, se puede calcular la resistencia nasal total.

\section{NASOFARINGE.}

Una de las principales funciones de la nasofaringe es servir como un tubo rígido que se abre al paso del aire. La nasofaringe también funciona como conducto para ventilación del oído medio a través de la trompas de Eustaquio y como vía de drenaje de la nariz y el oído medio, además de ser una cámara de resonancia importante en la producción de la voz.

\section{BUCOFARINGE.}

La boca y la faringe sirven principalmente como drenaje de la nasofaringe, así como de cámara de resonancia para la producción de la voz. Se encuentran abiertas para el paso libre del aire, excepto durante la deglución y el vómito. El tejido linfoide de la estructura nasofaringe tiene una función protectora, ya que remueve y destruye el material extraño que entra por vía bucal o nasal, además de participar en la formación de linfocitos $B$.

\section{LARINGE.}

Durante la respiración normal, las cuerdas vocales están en abducción y la resistencia es menor; al final de la inspiración, las cuerdas se encuentran en aducción aumentando la resistencia laríngea. La resistencia laríngea al paso del aire corresponde casi al $90 \%$ de la resistencia aérea total.

Durante la hipercapnia (aumento de la presión parcial de dióxido de carbono PaCO2 en sangre arterial por arriba de $46 \mathrm{mmHg}$ a nivel del mar) se amplía la glotis, 
aumenta la actividad eléctrica del músculo cricoaritenoideo posterior y disminuye la resistencia aérea de la laringe; esta caída es primero inspiratoria y luego espiratoria. La hipoxemia (disminución de la presión parcial de oxígeno $\mathrm{P}_{\mathrm{a}} \mathrm{O}_{2}$ en sangre arterial por debajo de $80 \mathrm{mmHg}$ a nivel del mar) también disminuye la resistencia laríngea.

La actividad física de la laringe depende de su musculatura intrínseca y se ha demostrado que la laringe participa en el control de tiempo de ventilación al modificar la duración de la espiración. Existen, además tres tipos de receptores pulmonares en la musculatura:

- De estiramiento.

- Irritativos.

- No mielinizados

Cada receptor se relaciona con alteraciones en la velocidad y profundidad de la respiración, el pulso y la tensión arterial. Los receptores de estiramiento producen durante la inflación pulmonar progresiva la disminución de la resistencia laríngea, además de inhibir la actividad física inspiratoria de los abductos laríngeos; este efecto desaparece al seccionar al nervio vago. Los receptores no mielinizados, producen un aumento de la resistencia laríngea. Mientras que los receptores irritativos, originan una disminución en la resistencia respiratoria de la laringe, sobre todo inspiratoria. La inhibición de los músculos inspiratorios de la respiración por los receptores de estiramiento se conoce como reflejo de Hering-Breuer.

\section{TRÁQUEA Y BRONQUIOS PRINCIPALES.}

La porción extra-torácica de la tráquea se encuentra en la línea media de la parte anterior del cuello. Después de entrar en el tórax, la tráquea se desvía ligeramente hacia la derecha. Las paredes traqueales son paralelas excepto por encima del origen del bronquio principal izquierdo. La tráquea está sostenida por anillos cartilaginosos en forma de herradura que se conectan por detrás mediante una membrana elástica.

La tráquea se divide en bronquios principales derecho e izquierdo, la carina, que es el ángulo de la bifurcación, varía de una persona a otra según la constitución 
corporal, pero siempre en un ángulo agudo. El bronquio principal izquierdo es más largo $(5 \mathrm{~cm}$.) que el derecho $(3 \mathrm{~cm}$.) [2].

\section{RAMAS LOBARES Y SEGMENTARIAS.}

Estar familiarizado con la anatomía de los cinco lóbulos y sus segmentos broncopulmonares no es solamente de utilidad académica, porque cada vez que se examina el tórax de un paciente y cada vez que se observa una radiografía del tórax, el examinador debe tener en cuenta los hallazgos en relación con su localización segmentaria.

Se llama segmento broncopulmonar a la cuña de tejido pulmonar que tiene un sólo bronquio segmentario y su arteria pulmonar correspondiente; su drenaje venoso es variable; las venas suelen drenar a más de un segmento. Cada uno funciona como una unidad más o menos independiente, aunque hay cierto grado de circulación colateral y ventilación entre segmentos adyacentes. Los segmentos tienen una forma bastante irregular y su tamaño es muy variable, están separados por barreras de tejido conjuntivo que apenas se pueden percibir a simple vista pero que se observan fácilmente en los cortes microscópicos.

Los segmentos pulmonares que tienen nombres (Figura 1.2 al 1.4) son los que cuentan con divisiones primarias de los bronquios lobares. La división subsecuente produce subsegmentos, muchos de ellos no son lo suficientemente uniformes para recibir una designación anatómica específica, aunque se han propuesto diversos sistemas de nomenclatura.

Esta anatomía segmentaria del pulmón tiene aplicaciones prácticas en muchos aspectos de la medicina respiratoria en especial cuando se efectúa broncoscopia y cuando se hace el diagnóstico diferencial de enfermedades particulares. Durante la exploración broncoscópica con el fibrobroncoscopio, deben identificarse todas las aberturas segmentarias y se puede entrar en muchas de ellas para inspeccionar los orificios y las porciones proximales de las vías respiratorias subsegmentarias 


\section{PuLMÓN DERECHO.}

El pulmón derecho está dividido en 3 lóbulos que originan diez segmentos: tres de ellos constituyen el lóbulo superior derecho; dos el lóbulo medio y cinco el lóbulo inferior derecho.

\section{LÓBULO SUPERIOR DERECHO.}

El bronquio del lóbulo superior emerge en sentido lateral en relación al bronquio derecho principal, cerca de la bifurcación de la tráquea, suele tener sólo $1 \mathrm{~cm}$. de longitud y luego se divide en tres bronquios segmentarios: el bronquio del segmento apical, denominado a veces segmento número 1, que asciende casi en sentido vertical; el bronquio segmentario posterior segmento número 2, que se dirige hacia atrás, por último el segmento anterior 3 , que se extiende hacia adelante y a menudo ligeramente hacia abajo.

\section{LÓBULO MEDIO.}

El bronquio de este lóbulo se origina en la superficie anterior del bronquio derecho principal. Su orificio está directamente enfrente del bronquio segmentario superior del lóbulo inferior derecho. Se extiende hacia adelante, hacia afuera y hacia abajo y se divide en dos bronquios segmentarios: La rama lateral (número 4), que lleva aire hacia la porción lateral y posterior del lóbulo medio, y la rama medial (número 5), que conduce aire hacia la porción anterior y medial.

\section{LÓBULO INFERIOR DERECHO.}

El segmento superior del lóbulo inferior (número 6) es anatómicamente distinto a los segmentos restantes o basales de este lóbulo. El bronquio segmentario superior nace directamente enfrente del bronquio del lóbulo medio, se extiende hacia atrás y hacia arriba y suele dar origen a tres bronquios subsegmentarios. A veces el segmento superior se divide en dos subsegmentos. El segmento superior del lóbulo inferior puede ser bastante grande y extenderse muy hacia arriba, por detrás casi hasta la punta del pulmón. 
1. Bronquio apical

2. Bronquio posterior

3. Bronquio anterior

4. Bronquio lateral

5. Bronquio medial

6. Bronquio superior

7. Bronquio basal medial

8. Bronquio basal anterior

9. Bronquio basal lateral

10. Bronquio basal posterior

1

Bronquio del lóbulo superior

Bronquio del lóbulo medio

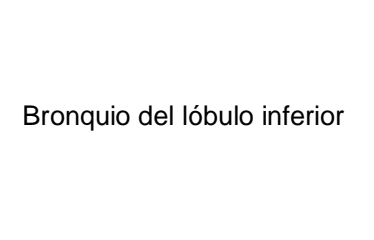

1 y 2

Bronquio apical posterior

Bronquio anterior

3

Bronquio lingual superior

4

Bronquio lingual inferior

5

Bronquio superior

6

Bronquio basal anterior medial

7 y 8

Bronquio basal lateral

Bronquio basal posterior

9

10

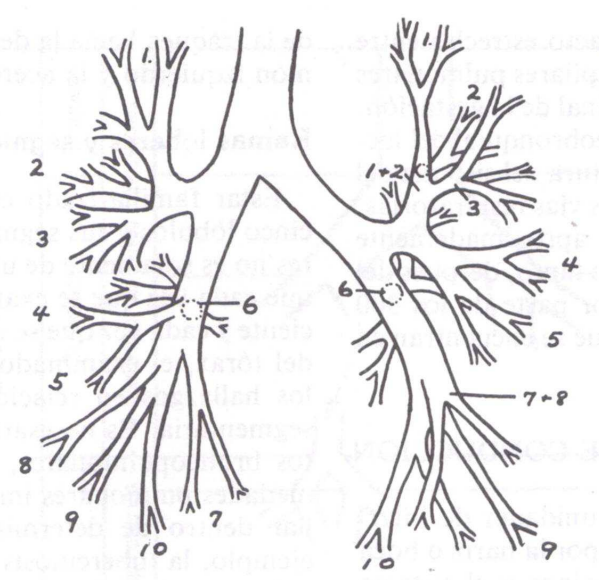

Figura 1.2 Sistema traqueobronquial normal. Representación esquemática del árbol traqueobronquial normal en la que se observa la numeración ordinaria de los bronquios segmentarios en una proyección posteroanterior.
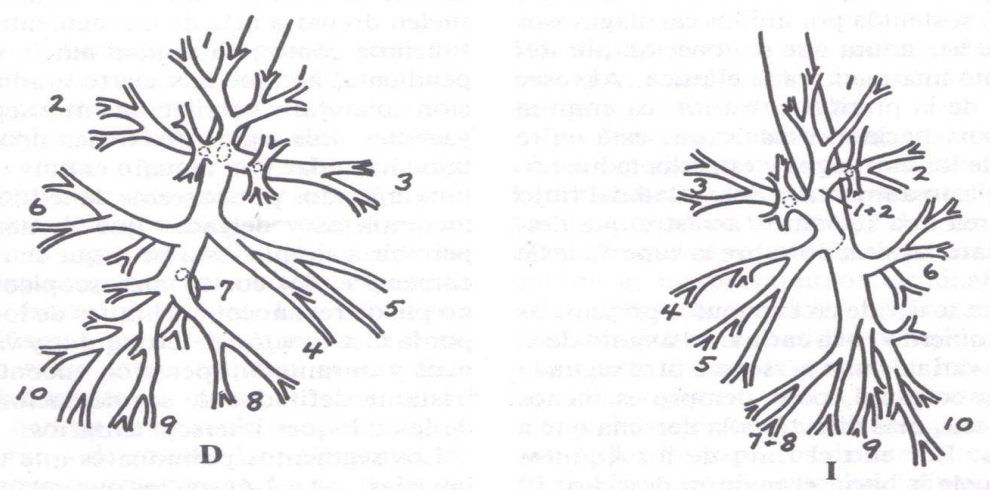

Figura 1.3 Árbol traqueobronquial normal. La numeración se refiere a los segmentos normales de los pulmones derecho (D) e izquierdo (I), según la lista que aparece en la figura 1.2 

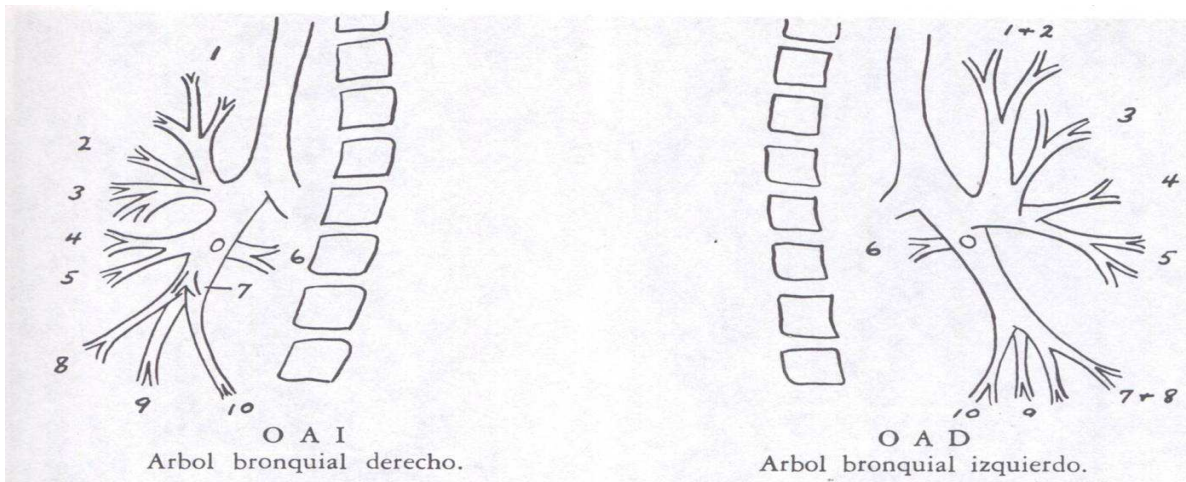

Figura 1.4 Árbol traqueobronquial normal. La numeración se refiere a los segmentos normales que en la figura 1.2, en proyecciones oblicua izquierda anterior (OIA) y oblicua derecha anterior (ODA).

El bronquio segmentario basal medial (número 7) suele ser el primer bronquio segmentario basal que aparece por debajo de la abertura del bronquio del lóbulo medio. Este bronquio lleva aire a los segmentos adyacentes al corazón y, en la nomenclatura antigua se denominaba bronquio cardiaco. El bronquio segmentario basal anterior (número 8) se extiende hacia adelante, hacia afuera y hacia abajo. El bronquio segmentario basal lateral (número 9) se extiende lateralmente por detrás del segmento medial del lóbulo medio. El segmento basal posterior (número 10) se identifica fácilmente en las radiografías laterales y se extiende hacia el surco posterior por detrás de la cúpula diafragmática, ubicado en relación medial y adyacente al segmento basal lateral.

\section{PULMÓN IZQUIERDO.}

Como ya se mencionó, el bronquio principal izquierdo es más largo que el derecho. El pulmón izquierdo tiene sólo dos lóbulos y su volumen total es un $20 \%$ menor que el del pulmón derecho. La diferencia de volumen entre ambos es debido al corazón.

\section{LÓBULO SUPERIOR IZQUIERDO.}

Equivale a el lóbulo superior y medio del lado derecho; la parte equivalente al lóbulo superior derecho suele llamarse división superior y la parte equivalente al lóbulo medio recibe el nombre de división singular (o inferior). 
El bronquio de la división superior suele tener dos ramas en vez de las tres que se observan en el lóbulo superior derecho. La primera de ellas, la apical posterior (números 1 y 2) suele dividirse en bronquios subsegmentarios apical y posterior, cuyas distribuciones son correspondientes a las de los segmentos apical y posterior del lado derecho. El segmento anterior (número 3) suele ser una división aparte.

El bronquio de la división singular, que corresponde al bronquio del lóbulo medio de la derecha, se divide en los dos bronquios segmentarios denominados superior (número 4) e inferior (número 5). La língula cuenta con bronquios que corren hacia abajo. La división singular se extiende hacia adelante y se observa mejor en las proyecciones laterales izquierdas, en las que la língula se sobrepone a la sombra cardiaca. Las proyecciones oblicuas anteriores derechas muestran también la língula con bastante claridad.

\section{LÓBULO INFERIOR IZQUIERDO.}

El bronquio principal a la izquierda, distal al origen de la rama del lóbulo superior izquierdo, se denomina bronquio del lóbulo inferior izquierdo (no hay bronquio intermediario a la izquierda, como sucede a la derecha). La primera rama del bronquio del lóbulo inferior izquierdo es la rama segmentaria superior (número 6), cuya distribución y patrón de ramificación sub segmentaria corresponden a su equivalente derecho. La rama basal izquierda del bronquio del lóbulo inferior izquierdo es el bronquio segmentario basal antero medial (números 7 y 8), que corresponde a los bronquios segmentarios basales medial y anterior del lado derecho. Los bronquios basales lateral (número 9) y posterior (número10) a la izquierda son semejantes a los del pulmón derecho.

\section{ESTRUCTURA DE LAS VÍAS RESPIRATORIAS}

Los bronquios experimentan divisiones cada vez más pequeñas, el número de "generaciones" depende de la longitud de la vía respiratoria, desde los bronquios segmentarios hasta las unidades de intercambio de aire (alvéolos). Los últimos y más pequeños bronquios de las vías del sistema de conducción de las que surgen las unidades respiratorias terminales, se denominan bronquiolos terminales. Los que están 
cercanos al hilio pueden ser alcanzados apenas por 10 ramas bronquiales, en tanto que quizá se requieran más de 25 ramificaciones para llegar a los bronquiolos terminales de las partes basales y periféricas del pulmón.

Los bronquios y las arterias pulmonares viajan siempre juntos por el pulmón; en cambio las venas, se encuentran tan apartadas de estas estructuras pares como es posible desde el punto de vista anatómico. Las grandes vías respiratorias y los vasos sanguíneos se hallan dentro de una túnica de tejido conjuntivo; en contraste, las ramas pequeñas (bronquiolos, arteriolas y vénulas) están en contacto directo con el parénquima pulmonar circundante.

Además de dar paso al aire hacia las unidades respiratorias terminales y de defender al pulmón, el calibre y la longitud de las vías de conducción varían pasivamente según los movimientos de expansión pulmonar. $Y$ lo que es más importante: las vías respiratorias contienen elementos musculares que reaccionan activamente a diversos estímulos neuro-humorales y químicos de origen fisiológico y patológico.

Los bronquios grandes y pequeños están sostenidos por un revestimiento de tejido fibroso; los grandes bronquios los estabiliza también un esqueleto externo de anillos cartilaginosos; estos anillos dan origen a láminas que se vuelven cada vez más discontinuas en las generaciones sucesivas y que acaban por desaparecer aproximadamente a la mitad del proceso (décima segunda generación) en el árbol traqueobronquial. A pesar de sus estructuras de sostén, las vías respiratorias son capaces de estrecharse considerablemente ante los estímulos neuro-humorales y los grandes bronquios se comprimen mucho durante la tos. Cuando fracasa el sistema fibroso de sostén, como por ejemplo en un paciente con enfisema avanzado, hay destrucción del parénquima pulmonar y de alvéolos, lo que resulta en atrapamiento aéreo y problemas para direccionar el flujo de aire al exterior del sistema.

\section{UNIDADES RESPIRATORIAS TERMINALES.}


Las unidades respiratorias terminales son más distales en relación con los bronquiolos terminales. Ya en estas unidades se lleva a cabo el intercambio gaseoso.

\section{LOBULILLOS.}

Son un grupo de tres a cinco bronquiolos terminales, cada uno con su unidad respiratoria terminal saliente. Los lobulillos contiguos están limitados de manera incompleta por tabiques de tejido conjuntivo, en los que hay conductos intercomunicantes que proporcionan ventilación colateral entre los lobulillos y las unidades respiratorias terminales vecinas. Estas estructuras son más importantes como puntos anatómicos de referencia que como unidades fisiológicas. Sus límites sirven para designar el enfisema como centrilobulillar o panlobulillar.

\section{UNIDADES RESPIRATORIAS.}

Las estructuras distales a los bronquiolos terminales, que solían llamarse "acinos" o "lobulillos primarios", se describen con más propiedad como unidades respiratorias terminales. La designación "unidad respiratoria" indica que en ellos se cumple la función primordial del pulmón, o sea captar el oxígeno y eliminar el bióxido de carbono. Las unidades tienen un típico patrón de ramificación variable, suele haber dos a cinco órdenes de bronquiolos respiratorios; a su vez, los últimos originan de dos a cinco órdenes de conductos alveolares. Cada conducto alveolar contiene de 10 a 16 alvéolos. El número total de alvéolos fluctúa según la edad y el tamaño corporal, pero la cifra que suele citarse es de 300 millones de alvéolos en el adulto de tamaño promedio. Esa cifra significa que los pulmones tienen una enorme superficie interna de entre 70 a $80 \mathrm{~m}^{2}$ aproximadamente más de 40 veces el área de superficie total de la piel. Como el $90 \%$ de la superficie alveolar está cubierta por capilares pulmonares, la superficie alvéolocapilar disponible para el intercambio gaseoso es de 60 a $70 \mathrm{~m}^{2}$.

\section{TABIQUE INTERALVEOLAR.}

La microscopia electrónica del pulmón humano normal muestra capilares suspendidos entre las dos capas epiteliales que forman el tabique interalveolar. Los capilares se abren camino por el tabique y encuentran primero un alveólo y a continuación otro. El endotelio capilar tiene relaciones anatómicas bastante diferentes 
con las dos capas epiteliales. Aproximadamente a la mitad del perímetro capilar, las membranas basales del epitelio alveolar y del endotelio capilar parecen estar fusionadas; esto define la porción "delgada" del tabique interalveolar en donde por ser menor la distancia entre el aire y la sangre; se produce el intercambio de aire. En cambio, las membranas basales del endotelio de la otra mitad del perímetro capilar y del epitelio vecino están separadas por un espacio intersticial; esto constituye la porción "gruesa" del tabique interalveolar, donde se realiza el intercambio de líquidos y solutos. De este modo se distinguen y separan anatómicamente entre sí las zonas para la transferencia de gases y la filtración de líquidos.

\section{PARED TORÁCICA.}

El tórax es la parte del cuerpo comprendida entre el cuello y el abdomen, e incluye los hombros, los huesos y los tejidos blandos sobre la pared torácica; éstas son las estructuras clave cuya movilidad, junto con la del diafragma hace que el aire entre y salga de los pulmones. El tórax está encerrado por una estructura ósea de costillas y tiene la forma de cono truncado. En la Figura 1.5 se ilustra el esqueleto torácico. Las costillas se articulan con la columna torácica por detrás y salvo la décima primera y décimo segunda, se unen con el esternón por delante. Las costillas 1 y 6 se articulan directamente con el esternón por medio de pequeñas prolongaciones de cartílago; las costillas 7 a 10 están conectadas entre sí y con el esternón por medio de un segmento común de cartílago.

Entre las costillas se encuentran dos bandas de músculos, los músculos intercostales externos e internos. Los primeros se extienden desde las articulaciones entre las costillas y los cuerpos vertebrales hasta el origen de los cartílagos costales. Los segundos se extienden desde el esternón hasta los ángulos de las costillas. Aunque es difícil analizar los movimientos de las costillas que resultan de la contracción de los músculos intercostales, se cree que los músculos intercostales externos y las porciones intercartilaginosas de los intercostales internos participan en la inspiración y que sólo las porciones interóseas de los intercostales internos cumplen una función espiratoria. Por tanto, los músculos intercostales son principalmente músculos inspiratorios. 
La respiración normal en reposo se efectúa al contraerse los músculos de la inspiración (diafragma e intercostales); la espiración es pasiva (es decir, no se requiere esfuerzo muscular). Cuando se necesitan cantidades mayores de aire, la espiración normal se vuelve más activa (intercostales internos) y se utilizan los músculos extratorácicos que contribuyen a la inspiración y la espiración; los músculos accesorios de la respiración situados en el cuello (esternocleidomastoideos y escalenos) y en el abdomen (recto, oblicuos externos e internos y transversos abdominales) sirven para incrementar el ritmo y la profundidad de las respiraciones.

\section{DiAFRAGMA.}

El diafragma (Figura 1.5) una hoja musculotendinosa que tiene importantes funciones anatómicas y fisiológicas: separa a la cavidad torácica y la abdominal y es una fuente poderosa de fuerza muscular inspiratoria. El diafragma tiene un tendón central semilunar, cuya concavidad se orienta hacia atrás, en dirección a la columna vertebral. La banda circular de fibras musculares que se inserta en el tendón se origina por delante desde el apéndice xifoides, alrededor de la pared torácica, desde las costillas séptima a duodécima y por detrás desde el primer al tercer cuerpos vertebrales lumbares a la derecha, y primero y segundo a la izquierda. La longitud de las fibras musculares varía; son más largas en la porción posterolateral, en donde se produce la mayor movilidad muscular.

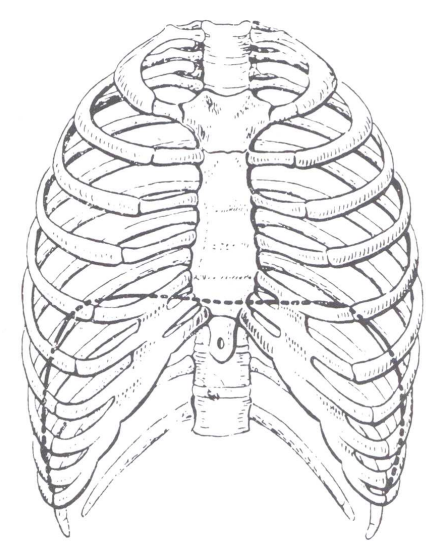

Figura 1.5 Esqueleto de tórax normal, en el que se observan costillas, esternón y columna torácica. La línea punteada indica la posición de la cúpula diafragmática durante la capacidad residual funcional. 


\section{LOS SONIDOS RESPIRATORIOS.}

Los sonidos respiratorios provenientes del tórax han sido considerados una fuente importante de información para el diagnóstico. Antes de la invención del estetoscopio, la forma de auscultar al paciente era colocando el oído en forma directa en su tórax, esta técnica no era muy bien aceptada por mujeres jóvenes, mas aún, era prácticamente imposible e impráctico escuchar los sonidos en el tórax de pacientes obesos. Tiempo después, René Theophil Laënnec inventó el estetoscopio en 1816 [3], lo que rápidamente fue aceptado por los médicos en Europa, ya que facilitaba las labores de auscultación en todo tipo de pacientes.

Para la auscultación del paciente se le debe de aislar de ruidos ambientales, para poder percibir los sonidos pulmonares, en posición sentada y con el tórax recto, en algunas ocasiones se lleva a cabo en posición supina debida a la condición precaria del paciente. En la auscultación el paciente puede ser instruido para respirar en forma normal, realizar respiraciones profundas, y otras maniobras como vocalización, provocar tos, espiraciones forzadas y maniobras de capacidad vital [4].

El origen de los sonidos respiratorios no es completamente claro, ya que se involucran múltiples mecanismos. El pulmón no puede generar sonidos sin un flujo de aire, aunque existan diferencias de presión entre las estructuras, o diferencias de volumen en el interior del tórax. Los sonidos respiratorios son generados probablemente por turbulencias de aire en el nivel lobular, bronquial o traqueal [3]. Se ha reportado que los sonidos respiratorios muestran diferencias en intensidad y contenido en frecuencia dependiendo entre otros factores de: (a) el sitio en donde son registrados, es decir en el tórax o en el cuello que corresponden a las vías aéreas superiores (tráquea, laringe); (b) la postura que mantienen los sujetos y, (c) la fase respiratoria (inspiración, espiración), entre otros.

\section{SONIDOS RESPIRATORIOS NORMALES Y ANORMALES.}

Los sonidos respiratorios normales están presentes sobre la caja torácica, extendiéndose por la parte superior hasta por encima de la clavícula y por la inferior 
hasta el diafragma. Cuando se auscultan los pulmones se pueden presentar varias categorías de sonidos: sonidos respiratorios normales, sonidos respiratorios disminuidos ó aumentados, y sonidos respiratorios anormales [5]. Los sonidos respiratorios ausentes o disminuidos son aquellos que no se perciben con el estetoscopio (son inaudibles) o que se escuchan con intensidad reducida. Esta categoría de sonidos refleja que hay una disminución del flujo de aire a una parte de los pulmones, tal como sucede con un enfisema, la presencia de aire o líquido alrededor de los pulmones, o un incremento del grosor de la pared torácica.

En relación a los sonidos respiratorios anormales existen varios tipos; los más comunes son los estertores, ronquidos y silibancias. Algunas veces se pueden escuchar las silibancias sin ayuda del estetoscopio debido a su magnitud. Los estertores son pequeños sonidos burbujeantes o crepitantes que se escuchan sobre un área pulmonar bien localizada y traduce que el aire entró y distendió el alveolo parcialmente colapsado, movilizando secreciones (moco, líquido, etc.). Los estertores reciben diversos calificativos: húmedos, secos, silibantes, agudos, finos, entre otros.

Los ronquidos son sonidos más audibles y graves. También se pueden describir como musicales, graves, groseros. Estos sonidos se producen cuando hay colapso parcial ó total de vía aérea superior.

Las silibancias son sonidos musicales agudos que se producen con la espiración cuando hay constricción (estrechamiento) de las vías respiratorias. Las causas comunes que provocan estos tipos de sonidos respiratorios anormales son:

- Tumores pulmonares.

- Obstrucción de las vías respiratorias por un cuerpo extraño.

- Tapones de moco en las vías respiratorias.

- Enfisema (distensión excesiva de los alvéolos).

- Asma. (inflamación del árbol bronquial).

- Obesidad (engrosamiento de la pared torácica).

- Personas muy musculosas (pared torácica gruesa).

- Síndrome de enfermedad respiratoria aguda del adulto (daño alveolar difuso). 
- Bronquiectasia. (dilataciones irreversibles de los bronquios mayores).

- Bronquitis crónica (producción de esputo en exceso).

- Fibrosis intersticial (obstrucción del sistema bronquial).

- Absceso pulmonar (infección necrosante del parénquima pulmonar).

- Neumonía. (inflamación del parénquima pulmonar).

- Edema pulmonar (aumento de la filtración de líquido a nivel capilar).

- Tuberculosis pulmonar. (enfermedad causada por la presencia de mycobacterium tuberculosis).

Los sonidos respiratorios anormales o adventicios pueden tener otras causas, como son: enfermedades sistémicas con afección pulmonar, reacción secundaria a medicamentos, la edad y el género que predispone a cierto tipo de sonidos anormales, las condiciones que lo agudizan y enfermedades asociadas.

\subsection{El Corazón y los Ruidos Cardiacos.}

\section{FISIOLOGÍA DEL MÚSCULO CARDIACO.}

El corazón que se muestra en la Figura 1.6 está formado por dos bombas separadas, un corazón derecho que bombea sangre a los pulmones y un corazón izquierdo que bombea sangre a los órganos periféricos. A su vez, cada uno de estos corazones es una bomba pulsátil de dos cavidades compuesta por una aurícula y un ventrículo, la aurícula funciona principalmente como una débil bomba cebadora del ventrículo, que ayuda a mover la sangre a su interior.

El ventrículo a su vez proporciona la principal fuerza que impulsa sangre a través de los pulmones o por la circulación periférica. En el corazón ciertos mecanismos especiales determinan el ritmo y transmiten los potenciales de acción por todo el músculo cardíaco, para causar el latido rítmico del corazón. 


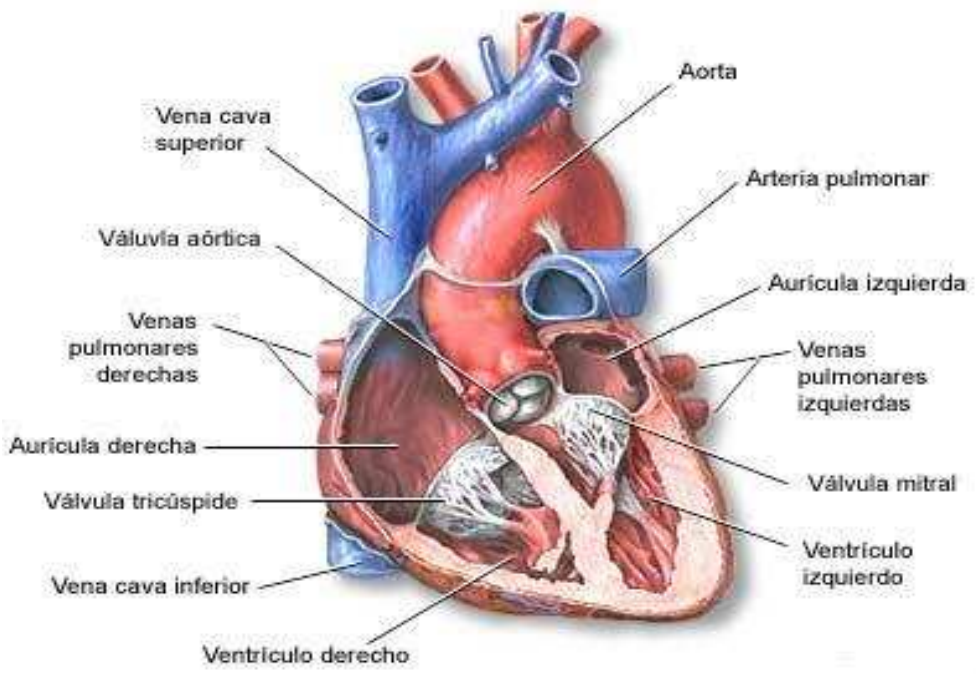

Figura 1.6 Anatomía del corazón

\section{EL CICLO CARDIACO.}

Los hechos que ocurren desde el comienzo de un latido hasta el comienzo del siguiente se conocen como ciclo cardiaco. El ciclo cardiaco consta de un período de relajación, denominado diástole, durante el cual el corazón se llena de sangre, seguido de un período de contracción llamado sístole.

\section{LOS RUIDOS CARDIACOS.}

\section{DESCRIPCIÓN DE LOS RUIDOS S1 y S2.}

Cuando se ausculta el corazón con un estetoscopio, no se escucha la apertura de las válvulas debido a que es un proceso relativamente lento, que no hace ruido. Sin embargo cuando cierran las válvulas, los bordes de las válvulas vibran debido a las diferencias de presión que se crean, produciendo ruidos que se trasmiten en todas direcciones por el tórax. Cuando los ventrículos se contraen, se escucha el primer ruido S1 causado por el cierre de las válvulas $A-V$, la vibración es de tono bajo y relativamente prolongada. Cuando las válvulas aórtica y pulmonar se cierran al final de la sístole, se ausculta un ruido relativamente rápido, debido a que esta válvula vibra en un período relativamente corto, este sonido se conoce como segundo ruido cardiaco S2 [4].

\section{DESCRIPCIÓN DE LOS RUIDOS S3 Y S4}


El ruido S3 es un sonido de baja frecuencia que tiene lugar en la diástole durante el periodo de llenado rápido ventricular, después de la apertura de las válvulas auriculoventriculares, coincidiendo con la máxima expansión del ápex, marca el llenado máximo ventricular. Puede ser normal (S3 fisiológico) o ser un signo de insuficiencia ventricular izquierda y derecha (galope diastólico o ventricular) o de la pericarditis constrictiva (de más alta frecuencia, acentuado y precoz).

Con respecto a S4, éste consiste en un sonido de baja frecuencia, presistólico, que coincide con la contracción auricular. A diferencia del S3, S4 es siempre patológico excepto en los niños. Es un sonido de llenado, producido por una contracción auricular acentuada en la fase de llenado ventricular activo. Existe un aumento de la presión diastólica final del ventrículo correspondiente.

Los ruidos cardiacos se registran en los siguientes lugares sobre la pared torácica:

Aórtica: Segundo espacio intercostal izquierdo

Pulmonar: Segundo espacio intercostal derecho

Mitral: Quinto espacio intercostal derecho hacia la línea axilar

Tricúspide: Quinto espacio intercostal izquierdo hacia el esternón

El llenado y vaciado de las cámaras del miocardio, el movimiento de sangre, apertura y cierre de válvulas, producen turbulencia, provocando vibraciones en el interior del corazón, transmitiéndose por las paredes y por los tejidos aledaños [6].

Es importante indicar que esta tesis se enfoca a reducir la presencia del primer y segundo ruido cardiaco, dado a su presencia relevante en los sonidos respiratorios adquiridos en sujetos sanos y en enfermos. 


\section{CAPÍTULO 2 PLANTEAMIENTO DEL PROBLEMA Y ANTECEDENTES}

\section{1 ¿Porqué los Ruidos Cardiacos Representan una Interferencia para el Análisis de los Sonidos Respiratorios?.}

La auscultación pulmonar presenta ventajas como procedimiento de diagnóstico clínico, ya que por su facilidad de realización y ausencia de riesgos es factible de practicar, independiente de la edad del paciente y de su estado de salud. Sin embargo, en la práctica clínica, la descripción de los hallazgos durante la auscultación depende en gran medida de la experiencia del médico, lo que complica el seguimiento de la evolución de los pacientes, debido al carácter subjetivo de las descripciones. Para eliminar la subjetividad del método clínico actual, se ha planteado el uso de sistemas de análisis computarizado que permitan una evaluación cuantitativa de los sonidos respiratorios[3].

Estos esfuerzos generarían una herramienta de tipo no invasiva para el diagnóstico de enfermedades pulmonares desde un punto de vista funcional. Hay que considerar que los sonidos respiratorios registrados en la superficie torácica no sólo representan los sonidos generados en las vías áreas, también representan el efecto que el parénquima pulmonar tiene sobre el viaje de las ondas acústicas y las características del sistema de adquisición, lo cual incluye al sensor. Sin embargo, para la caracterización cuantitativa se requiere eliminar las señales de interferencia que se añaden a la señal adquirida como son: los ruidos cardiacos, los ruidos producidos por la contracción muscular, el ruido ambiental, los ruidos provocados por el contacto entre la piel y el micrófono, entre otros [7].

De las interferencias, la más evidente es la provocada por los ruidos cardiacos los cuales son producidos por diferentes eventos dinámicos valvulares y por el flujo sanguíneo [4]. Las vibraciones producidas por dichos eventos, viajan a través de los tejidos adyacentes hasta la pared torácica, percibiéndose con el estetoscopio en diferentes puntos en la superficie torácica. Por lo tanto, los ruidos cardiacos se encuentran inmersos en los sonidos respiratorios, lo que implica un traslape en tiempo de las informaciones acústicas Figura 2.1. En particular, los ruidos cardiacos 
comprenden componentes espectrales que pueden estar en el rango de los 10 hasta los $400 \mathrm{~Hz}$ [6] y sus características presentan cambios debido a:

- La distancia entre la posición del miocardio y el micrófono de adquisición de la señal acústica.

- Sitio de adquisición en el tórax.

- Cambios por las diferencias anatómicas entre sujetos.

Se cuenta con un cierto conocimiento de las características de los ruidos cardiacos en los dominios del tiempo y la frecuencia así como sus cambios morfológicos cuando se adquieren en diversos puntos sobre el tórax humano. En [8], por ejemplo, se realiza un análisis tiempo-frecuencia del primer ruido cardiaco en apnea espiratoria en 27 puntos diferentes del tórax humano principalmente considerando la región definida por el hemitórax izquierdo. El objetivo es la caracterización del primer ruido cardiaco así como el proponer una herramienta diferente de análisis. Las observaciones en este trabajo indican que a pesar de que los puntos de adquisición no estuvieron separados por más de $6 \mathrm{~cm}$, se advierte un importante cambio morfológico en el primer ruido, con relación a su amplitud y contenido en frecuencia. Los autores indican que diferentes regiones en el tórax poseen diferentes ventanas de observación del corazón y por lo tanto, no es descabellado pensar que el contenido en frecuencia del primer ruido cardiaco sea diferente de acuerdo al sitio de registro.

En [9] se efectúa un análisis de la morfología de los ruidos cardiacos en dos posiciones distales, válvula aórtica y antebrazo, y se concluye que existen variaciones reflejadas sobre todo, en el segundo ruido cardiaco, debido a la zona de adquisición sobre el tórax. En [10] se realizan representaciones tiempo-frecuencia de los ruidos cardiacos durante diferentes condiciones respiratorias, es decir, en respiración normal, con inspiración sostenida y con espiración sostenida. Los ruidos cardiacos adquiridos, en condiciones de apnea y respiración, fueron filtrados entre 10 y $750 \mathrm{~Hz}$ y posteriormente, se aplicó la transformada wavelet para obtener su representación tiempo-frecuencia. En condiciones de apnea, y para sujetos sanos, se observa que la 
morfología temporal de los ruidos cardiacos se modifica de sujeto a sujeto y estos cambios se observan más claramente en las representaciones T-F. Posteriormente, se muestran las representaciones T-F para un paciente, con la válvula aórtica con problemas de estenosis, para tres ciclos cardiacos consecutivos en apnea y respiración. Se observa que durante la apnea las características de los ruidos cardiacos no se ven modificadas relevantemente, pero no así en respiración. Comparando las representaciones tiempo-frecuencia de los ruidos cardiacos en estas diferentes maniobras, los autores observan que en conjunto proveen información extra del comportamiento de los ruidos cardiacos que puede no ser obvia en registros en respiración normal.

En el dominio de la frecuencia los sonidos respiratorios abarcan componentes espectrales que van desde las frecuencias bajas hasta los $2000 \mathrm{~Hz}$, en el caso de los sonidos anormales. La densidad de potencia espectral de los sonidos respiratorios es de banda ancha con una potencia que decrece con la frecuencia[3]. Además, la intensidad del sonido respiratorio varía con el flujo respiratorio, con el sitio de adquisición en el tórax y con la complexión del sujeto. Varios investigadores han realizado estudios para determinar como varía la morfología del sonido respiratorio con el flujo, mostrando que existe una correlación entre la potencia de la señal de sonido respiratorio y el flujo y entre la media de la amplitud de la señal de sonido respiratorio y flujo [11]. Por otra parte, para los sonidos en tráquea y caja torácica, no se ha encontrando correlación entre el espectro de frecuencias y el flujo respiratorio para la fase de espiración, pero si una gran correlación sobre todo para bajas frecuencias, en la fase de inspiración [12]. Por lo tanto, los sonidos inspiratorios difieren de los espiratorios en magnitud y contenido en frecuencia.

Hay que puntualizar que el traslape en frecuencia entre los ruidos cardiacos y los sonidos respiratorios en el rango espectral de las frecuencias bajas ha impedido detectar con precisión el límite espectral inferior de los sonidos respiratorios. Así también se debe señalar que ambas señales acústicas, las respiratorias y las cardiacas, deben de considerarse señales no-estacionarias para efectos del procesamiento adecuado [8]. 


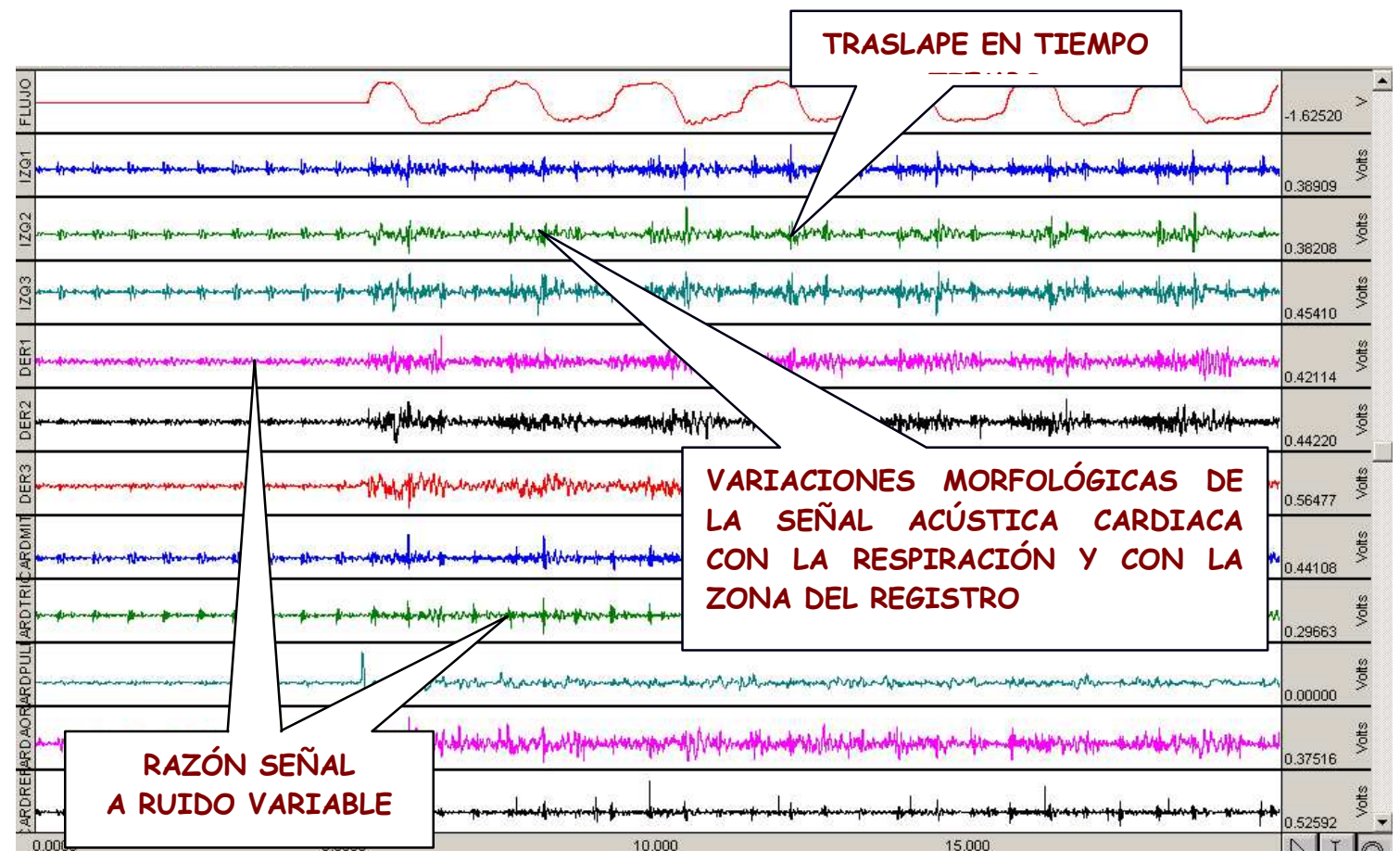

TIEMPO (segundos)

Figura 2.1 Señal de flujo respiratorio y multicanales de sonido a un flujo de $2 \mathrm{~L} / \mathrm{seg}$.

Los primeros registros de los sonidos durante la respiración pueden referirse a Martini y Müller[13], quienes en 1923 reportaron los componentes en frecuencia de sonidos bronquiales al analizar señales impresas sobre un tambor fotográfico. En 1955, Mckusick [14] presenta un novedoso método espectrográfico que permitía el análisis simultáneo de las características amplitud-tiempo y tiempo-frecuencia de los sonidos respiratorios. En las dos últimas décadas, los avances en la tecnología han permitido desarrollar sistemas computacionales que han mejorado la calidad de la adquisición, el análisis cuantitativo y la representación visual de los sonidos respiratorios, aspectos de relevancia para extraer características de los sonidos respiratorios que apoyen al diagnóstico médico [15]. Pasterkamp presenta una revisión general de los trabajos realizados por la comunidad dedicada al estudio de los sonidos respiratorios desde los esfuerzos de nomenclatura hasta las conclusiones respecto del origen de los sonidos respiratorios [3]. De hecho, la Comunidad Europea, lanzó un proyecto llamado "Computerized Respiratory Sound Analysis" (CORSA), con el objetivo de desarrollar 
estándares para la investigación y práctica médica en el campo del análisis de los sonidos respiratorios [16].

Últimamente ha existido un interés en los sistemas multicanales para el análisis del sonido respiratorio en distintos puntos de la pared torácica o para visualizar las diferentes señales acústicas por medio de imágenes de superficie utilizando funciones de interpolación para obtener imágenes con mejor resolución espacial de la información acústicas. El propósito de la imagenología acústica es evaluar la distribución del sonido respiratorio, el contenido en frecuencia y la ventilación en sujetos sanos y sujetos enfermos. En estos esfuerzos, la eliminación de los ruidos cardiacos adquiere mayor relevancia para un análisis de los sonidos respiratorios puros [17].

\subsection{Otros Intentos en la Reducción de la Interferencia Acústica Cardiaca}

Los ruidos cardiacos representan una señal de interferencia evidente durante la adquisición de los sonidos respiratorios, la reducción de la señal de interferencia podría mejorar el análisis de los sonidos respiratorios con propósitos de extracción de información cuantitativa y posterior clasificación, determinación de fuentes sonoras, etc.

Los intentos por reducir la presencia de los ruidos cardiacos se pueden dividir en determinísticos y estocásticos. Por ejemplo, se ha aplicado el filtrado determinístico pasa-altas del tipo no recursivo en el dominio o escala de tiempo original de la señal, con frecuencias de corte de 75 ó $100 \mathrm{~Hz}$, con la desventaja de perder información valiosa en bajas frecuencias del sonido respiratorio, dado el traslape espectral que ya se ha mencionado.

En el intento estocástico se ha utilizado el filtrado adaptativo en el dominio original de la señal y vía la combinación de diversas técnicas de representación tiempofrecuencia con filtrado adaptativo, entre otros. Los algoritmos utilizados para la actualización de los pesos del filtro adaptativo incluyen el least mean squares (LMS) (ver apéndice A.2), el recursive least squares (RLS) y el fast transversal filter (FTF). 
mientras que el esquema utilizado es el de eliminación de interferencias (ver apéndice A.2).

Una ventaja importante del filtrado adaptativo es que éste no requiere información a priori de las señales involucradas. Sin embargo, se requiere construir una señal de referencia y alinear la señal de referencia con la señal de interferencia a procesar, siendo la etapa de alineación complicada de llevar a cabo dado la relación señal a ruido entre la señal de interferencia y el sonido respiratorio en el dominio original. En [18] se construyó una señal de referencia que contenía información temporal para procesar el primer y segundo ruido cardiaco de la señal de interferencia a partir del complejo QRS de la señal electrocardiográfica. El tiempo entre complejos se consideró constante, sin embargo este intento no proporcionó resultados adecuados ya que existen variaciones de tiempo entre el primer y segundo ruido cardiaco de interferencia, así como alteraciones en la frecuencia cardiaca. Además de que la teoría de filtros adaptables requiere que la señal de interferencia y la de referencia sean altamente correlacionadas, estas señales deberían ser del mismo tipo, en el caso de esta tesis, ambas señales son acústicas.

Para evitar la adquisición de señales adicionales, como el ECG, en [19] se elaboró un esquema para construir la señal de referencia por medio de la combinación de filtros determinísticos pasa-bajas de la señal adquirida y la detección de los inicios y finales de los ruidos cardiacos filtrados obtenidos de esta forma. La señal de referencia incluyó los segmentos de información cardiaca filtrada entre los inicios y finales detectados y el resto se consideró cero. Sin embargo, se obtuvo una baja a moderada eliminación del ruido cardiaco quizás debido a que las señales cardiacas filtradas a partir de la señal adquirida, el cual, requiere de la selección adecuada de la frecuencia de corte para no incluir componentes respiratorios, lo cual depende a su vez del sitio de adquisición.

Por otra parte, para evitar la alineación entre señales de referencia e interferencia, se propuso utilizar el filtro Kalman, y un modelo autorregresivo para la señal de interferencia cardiaca. Los coeficientes del modelo AR se obtienen de un segmento de información cardiaca seleccionado de la etapa de apnea del registro 
acústico. Se establecen las ecuaciones necesarias para el filtro Kalman considerando al sonido respiratorio como un ruido aditivo de color y al ruido cardiaco como la señal de interés. El algoritmo se aplica a señales simuladas con diferentes relaciones señal cardiaca a ruido (sonido respiratorio) [20]. La desventaja de utilizar esta propuesta es la necesidad de contar con información estadística a priori de las señales acústicas cardiacas para estimar los coeficientes del modelo AR, además de que el algoritmo de Kalman es computacionalmente complejo.

En la referencia [21] se emplea la transformada wavelet para procesar las señales involucradas, y se explora el desempeño del algoritmo propuesto tanto en señales simuladas como en señales adquiridas. Es de hacer notar que este trabajo es uno de los artículos que forman parte de los antecedentes directos de esta tesis. La metodología propuesta estima los retrasos entre la señal de interferencia y la señal de referencia en cada sub-banda de la descomposición wavelet utilizando dos mecanismos para minimizar el error cuadrático entre las señales. Para actualizar el valor de los retrasos (o alineación requerida) se usa un algoritmo de optimización no lineal llamado Levenberg-Marquard, mientras que otro algoritmo adaptativo, con base en el block fast transversal filter, actualiza los coeficientes de un filtro adaptativo para eliminación de la interferencia cardiaca. La metodología posee ciertas ventajas como incorporar múltiple información complementaria en diferentes sub-bandas y es robusto en presencia de ruido. Sin embargo, la aplicación de la transformada wavelet implica la descomposición de la señal en sub-bandas, por lo que se debe determinar la mejor wavelet, así como determinar hasta qué nivel de descomposición es necesario realizar para cada señal de interés.

Otro esfuerzo siguió con la propuesta de aplicar filtros adaptativos, donde se obtienen señales acústicas de la parte anterior derecha del tórax para tres diferentes flujos (bajo, medio y alto) de seis sujetos sanos. Para eliminar la señal de ruido cardiaco se utiliza un filtrado adaptativo bajo el algoritmo RLS y con el esquema de eliminación de interferencias. Nuevamente la selección de la señal de referencia se dificulta y en este trabajo, se obtuvo la señal a partir de filtrar la señal adquirida de forma pasabanda entre 20 y $300 \mathrm{~Hz}$. Se compararon las potencias obtenidas en cuatro diferentes bandas entre 20 y $300 \mathrm{~Hz}$ entre la señal filtrada y la señal sin filtrar. Al parecer los resultados son prometedores a flujos bajos [19]. 


\section{CAPÍTULO 3 JUSTIFICACIÓN DE LA METODOLOGÍA PROPUESTA Y OBJETIVOS}

\subsection{Justificación de la Metodología Propuesta.}

Dadas las condiciones de las señales involucradas en esta tesis, es decir, del ruido cardiaco (RC) y del sonido respiratorio (SR), y de la instrumentación empleada, a saber:

- Traslape temporal y espectral de señales no estacionarias: RC y SR.

- Variación del SNR, para el RC, en los diferentes sitios de adquisición en el tórax en sistemas de adquisición multicanales.

- Posibles cambios morfológicos del RC con la respiración.

- La no adquisición de señales adicionales a las acústicas.

Se requiere:

- El uso de filtrado adaptativo para contender con el traslape espectral y temporal.

- El uso de técnicas de descomposición de señales no estacionarias para mejorar la SNR para el RC y para estimar la posición de la interferencia cardiaca en el dominio de la descomposición. En particular la técnica de empirical mode decomposition (EMD) es un algoritmo que está diseñado para contender con señales no-estacionarias y no lineales y que tiene el gran atractivo de ser una técnica adaptable. Además, otro elemento interesante es que se conoce que el desempeño del algoritmo de adaptación de los pesos para un filtro adaptativo mejora en el dominio de la descomposición.

- El uso de técnicas no lineales que permitan contender con los posibles cambios morfológicos de RC. En esta tesis surge el problema de que a pesar de que las señales registran un mismo fenómeno, no conservan la misma morfología. En particular, en las señales acústicas cardiacas se observan cambios de morfología entre la etapa de apnea y la de respiración, provocados posiblemente por la influencia de la respiración. Esto hace que las señales de interferencia y referencia cardiaca posiblemente no se relacionen linealmente. Una de las 
técnicas usadas para realizar la alineación de dos señales es la llamada técnica de Time Warping.

\subsection{Hipótesis.}

Una metodología de procesamiento basada en la descomposición de modos empíricos en conjunto con el filtrado adaptativo, en el dominio de la descomposición, reducirá en un porcentaje significativo la presencia de los ruidos cardiacos en la señales multicanales adquiridas para el análisis posterior de los sonidos respiratorios.

\subsection{Objetivo General.}

Proponer y evaluar una metodología alternativa, basada en el EMD y filtrado adaptativo, para la reducción de interferencias acústicas cardiacas con mínimos efectos para el contenido en frecuencia de los sonidos respiratorios que permita su análisis posterior.

\subsection{Objetivos Particulares.}

- Analizar cualitativamente la distribución de información espacio-temporal de los ruidos cardiacos y sonidos respiratorios en la pared torácica.

- Determinar de forma cualitativa la influencia del flujo respiratorio sobre las características temporales de los sonidos respiratorios y los ruidos cardiacos.

- Validar mediante medidas de desempeño la metodología propuesta en señales acústicas simuladas y adquiridas.

- Determinar las ventajas y desventajas, o limitaciones, de la metodología propuesta. 


\section{CAPÍTULO 4 METODOLOGÍA PROPUESTA}

\subsection{Esquema para la Cancelación de la Interferencia Acústica Cardiaca.}
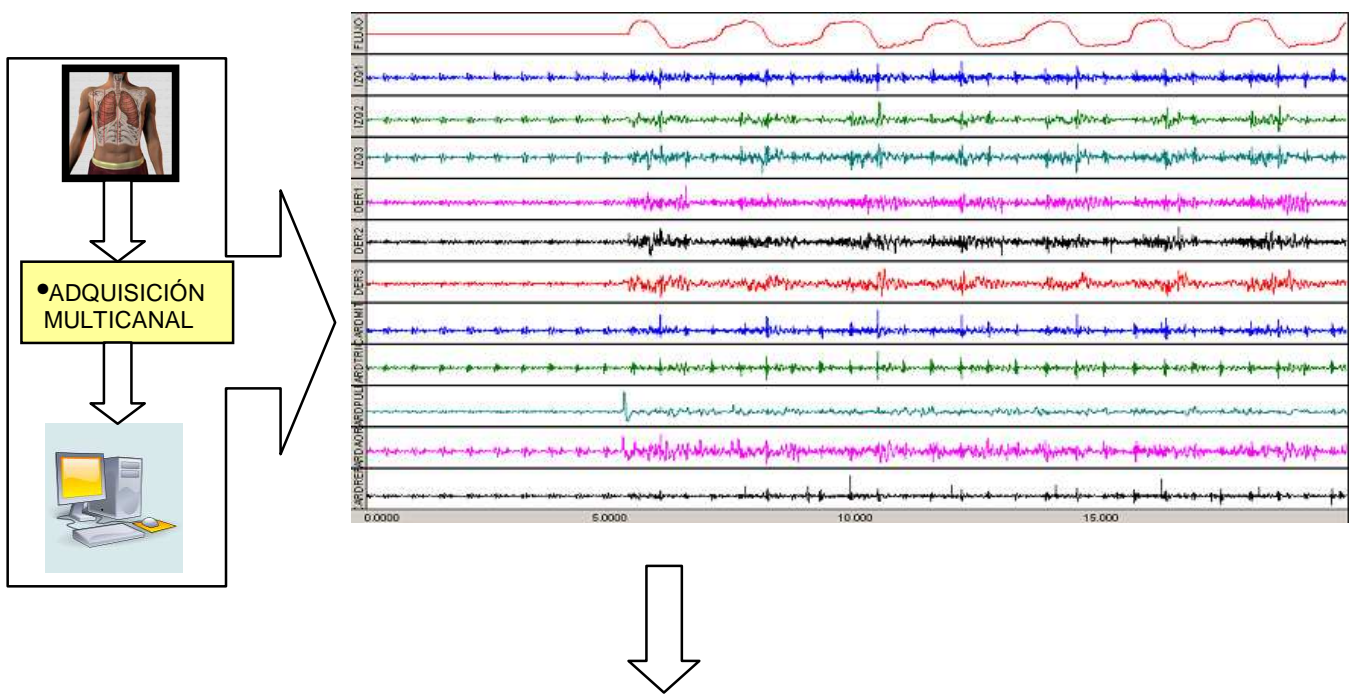

PREPROCESAMIENTO

- EMD de la información acústica.

- Análisis de modos de oscilación (IMFs).

- ¿Modos de oscilación con información acústica cardiaca?.

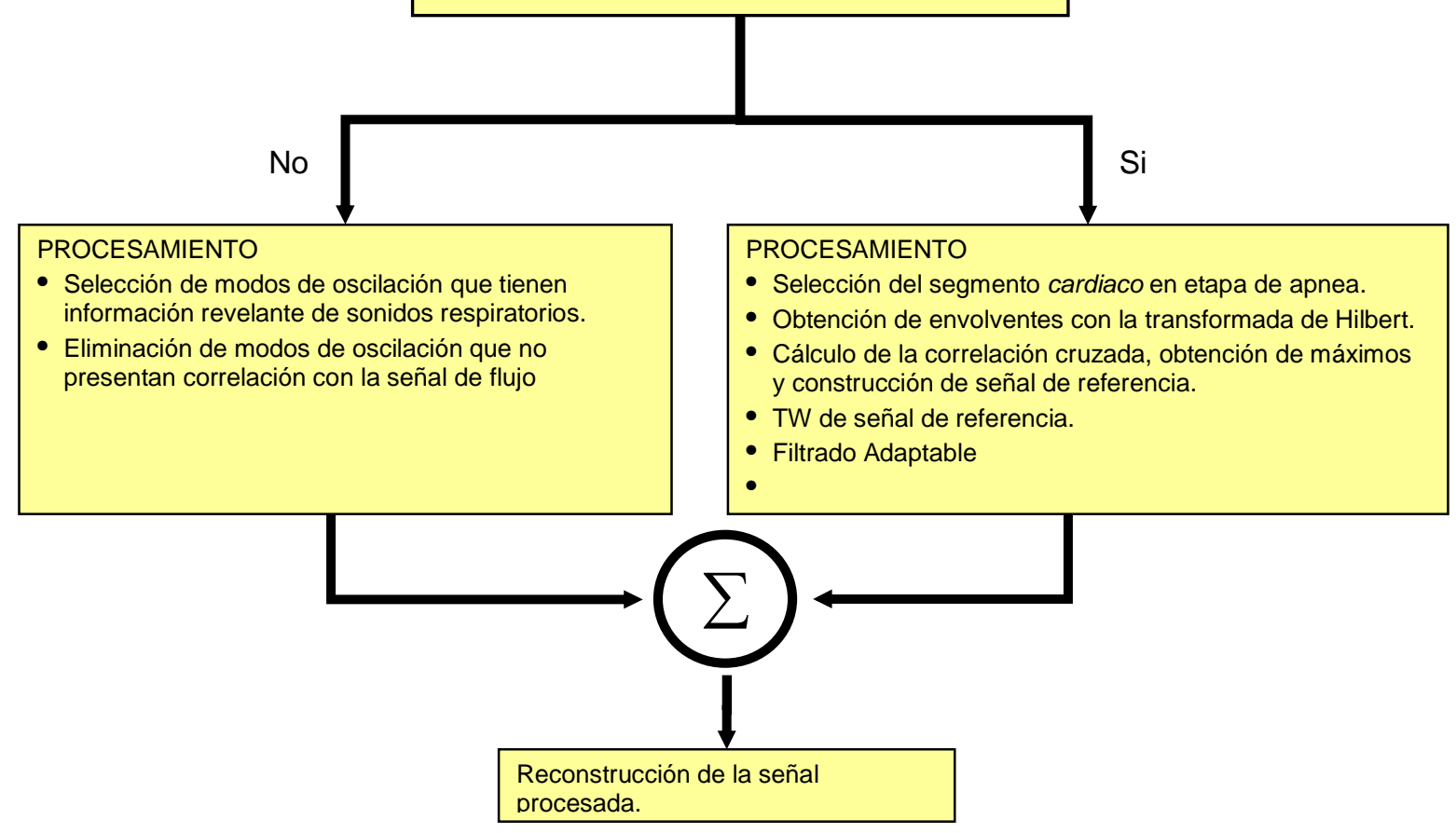

Figura 4.1 Diagrama a bloques de la metodología propuesta. 
En el diagrama a bloques de la Figura 4.1 se presenta la metodología propuesta que se describe a continuación en forma detallada.

\subsubsection{Descomposición de la Señal de Interés en Modos de Oscilación.}

Las señales acústicas adquiridas de forma multicanal, se descomponen mediante el algoritmo EMD [22] como se describe en la sección A.2.

\subsubsection{Creación de la Señal de Referencia $u(n)$.}

Una vez obtenidos los modos de oscilación de las señales adquiridas, se procesan los datos para minimizar los componentes de los ruidos cardiacos. El procesamiento se compone de los siguientes pasos:

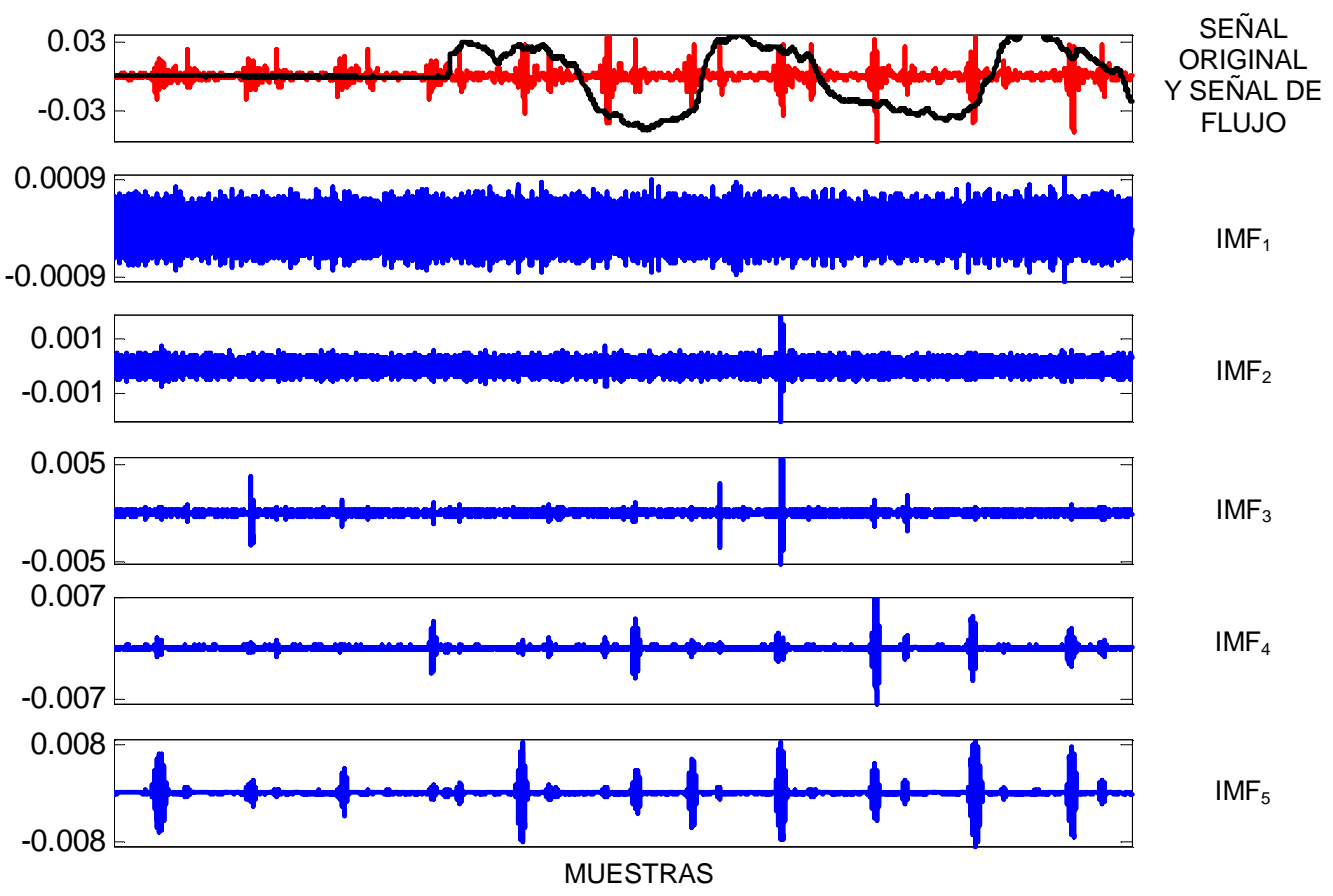

Figura 4.2 IMFs 1 a 5 de la señal acústica adquirida cerca del corazón que corresponde a la zona de auscultación de la válvula mitral.

1. Selección de IMFs. De los modos de oscilación obtenidos se eligen aquellos modos donde se visualizan componentes cuasi-periódicos temporalmente coincidentes con los ruidos cardiacos de la señal adquirida en el dominio original. Obsérvese las gráficas de la Figura 4.2 a la 4.4, en donde se muestra la descomposición completa 
de una de las señales adquiridas (la señal acústica original y la señal de flujo respiratorio se presentan en la parte superior de cada gráfica). En las primeros modos no aparecen componentes cuasi-periódicos, mientras que en modos posteriores se observan dichos componentes (ver Figura 4.2 y comparar IMF1 versus IMF6).

2. Selección del segmento de información cardiaca en la etapa de apnea. Una vez elegido manualmente el segmento cardiaco en la etapa de apnea, éste se utiliza para construir la señal de referencia (observe la Figura 4.5). El criterio de selección para el segmento incluye el que presente una amplitud importante y que sea temporalmente coincidente con los ruidos cardiacos de la señal en el dominio original.

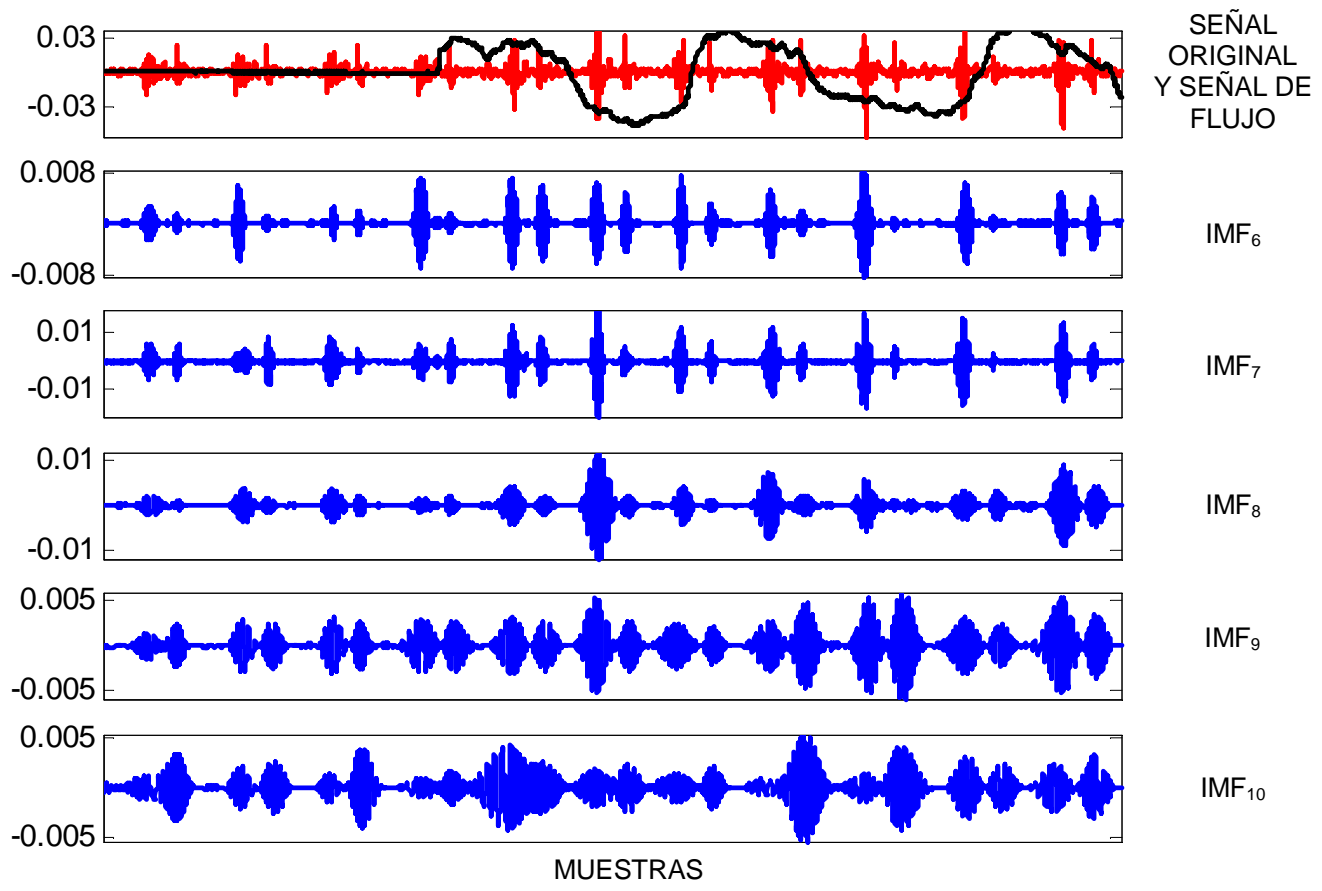

Figura 4.3 IMFs 6 a 10 de la señal acústica adquirida cerca del corazón que corresponde a la zona de auscultación de la válvula mitral. 


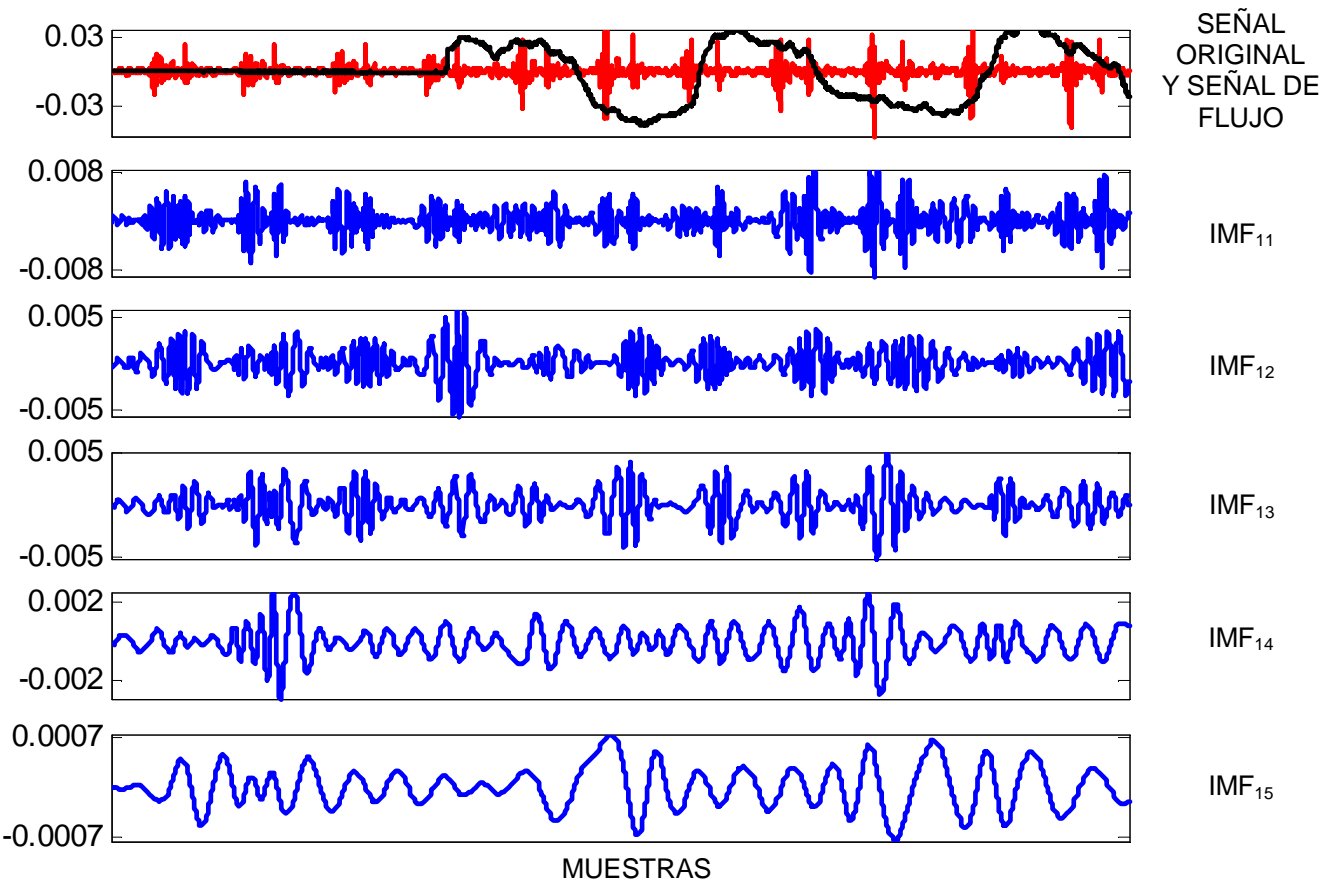

Figura 4.4 IMFs 11 a 15 de la señal acústica adquirida cerca del corazón que corresponde a la zona de auscultación de la válvula mitral.

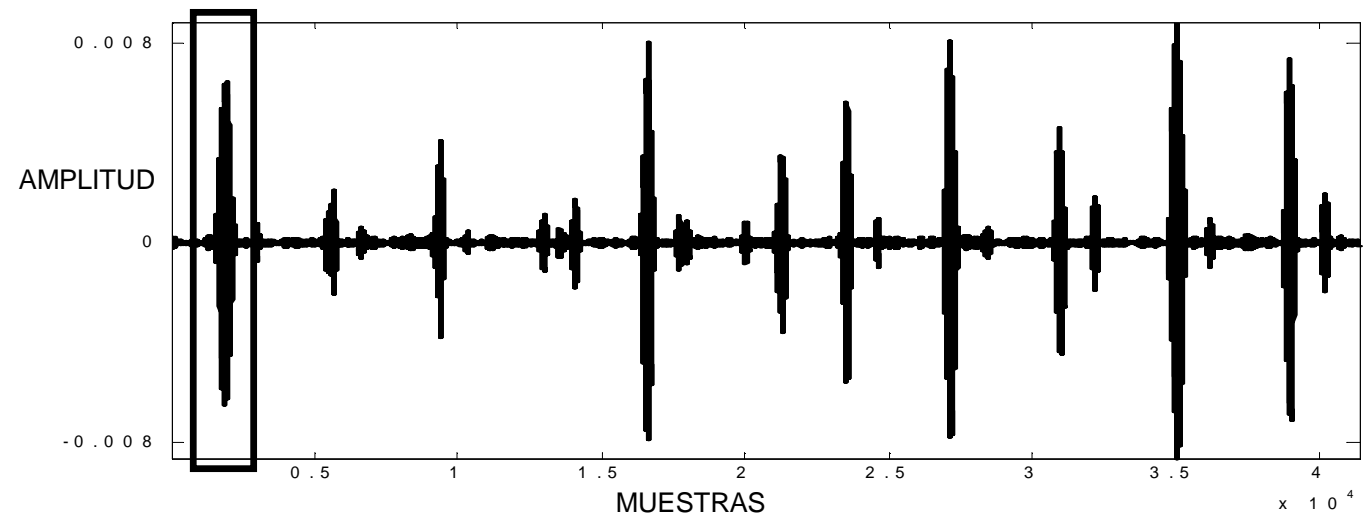

Figura 4.5 $\mathrm{IMF}_{6}$ de la Figura 4.2 con componentes cuasi-periódicos pertenecientes a ruidos cardiacos. El recuadro a la izquierda indica el segmento de apnea que se utilizará para generar la señal de referencia. 

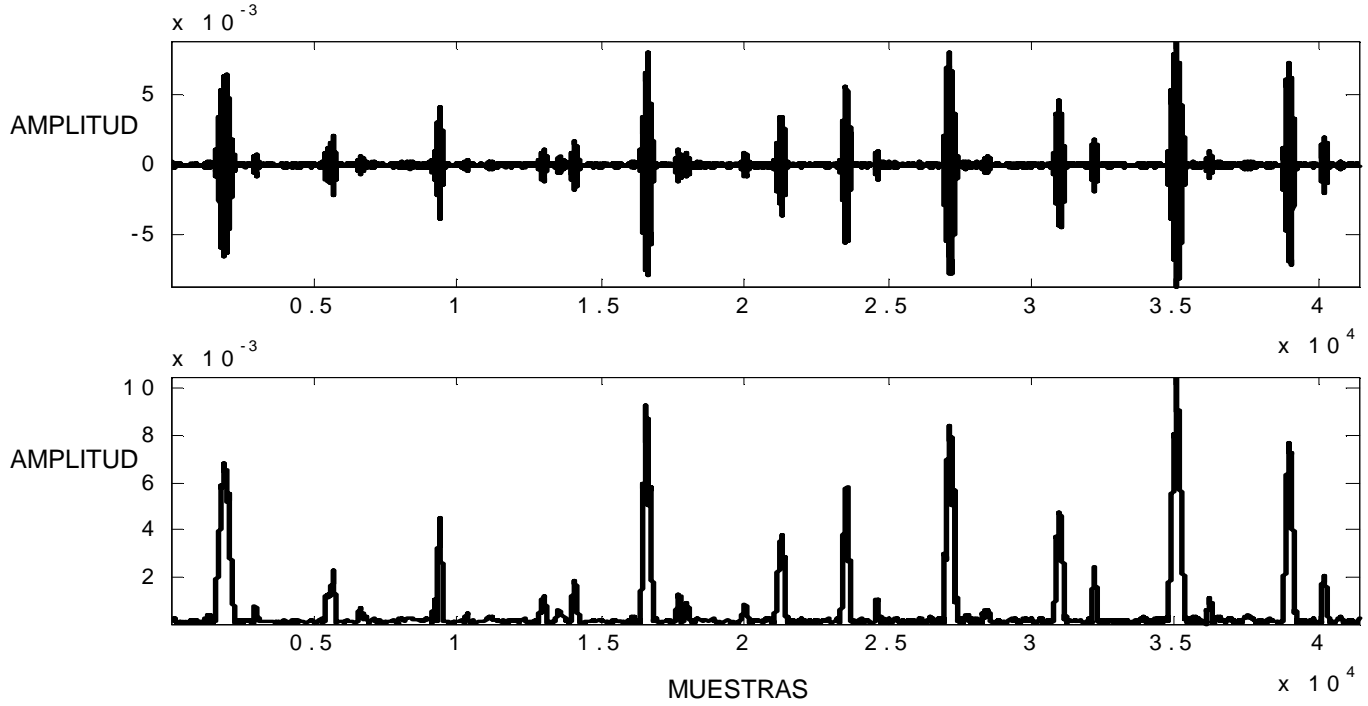

Figura 4.6 Modo de oscilación seleccionado con componentes cuasi-periódicos pertenecientes a ruidos cardiacos. La gráfica superior representa el $\mathrm{IMF}_{6}$ y la inferior su envolvente vía la transformada de Hilbert.

3. Aplicación de la transformada de Hilbert (tH). Se aplica la transformada de Hilbert para obtener la envolvente, vía el valor absoluto de la $\mathrm{tH}$, del segmento seleccionado y del correspondiente modo de oscilación a procesar (Figura 4.6).

4. Se obtiene la correlación cruzada entre la envolvente del segmento seleccionado y la envolvente del resto del IMF para extraer las posiciones en donde la función de correlación es máxima (obsérvese la Figura 4.7).

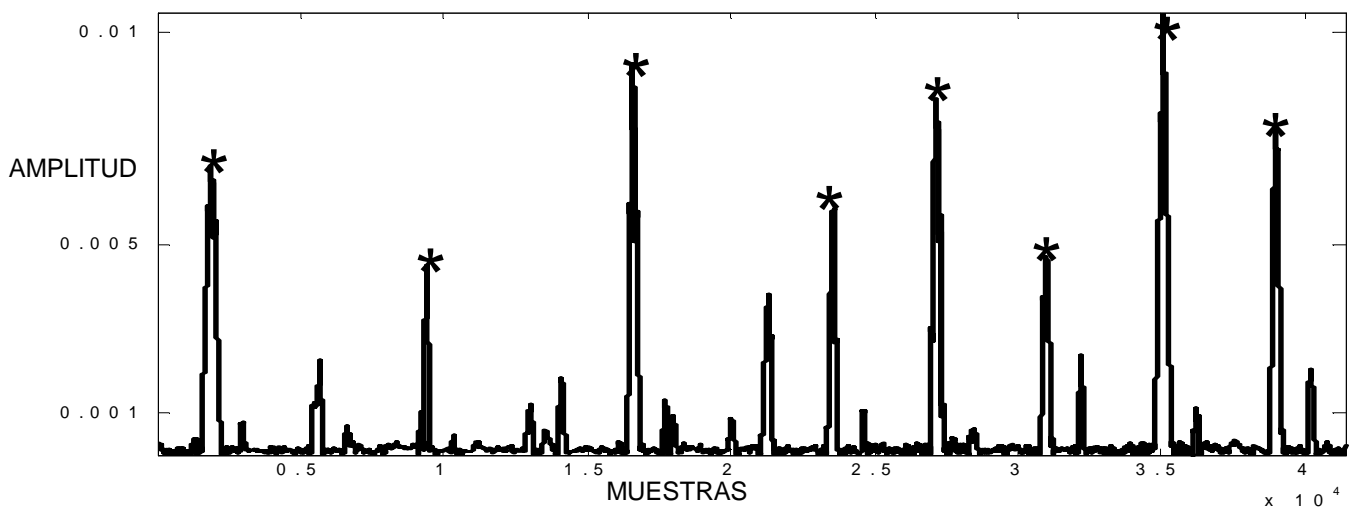

Figura 4.7 Se observa la misma envolvente que en la figura inferior 4.6 y con asteriscos se indican los máximos localizados de la función de correlación cruzada. 


\subsubsection{Uso del Time Warping, vía el COW.}

1. Señal no alineada. Se genera la señal de referencia $u$ insertando el segmento seleccionado en apnea, en las posiciones indicadas por los valores máximos de la función de correlación, denominada SR1.
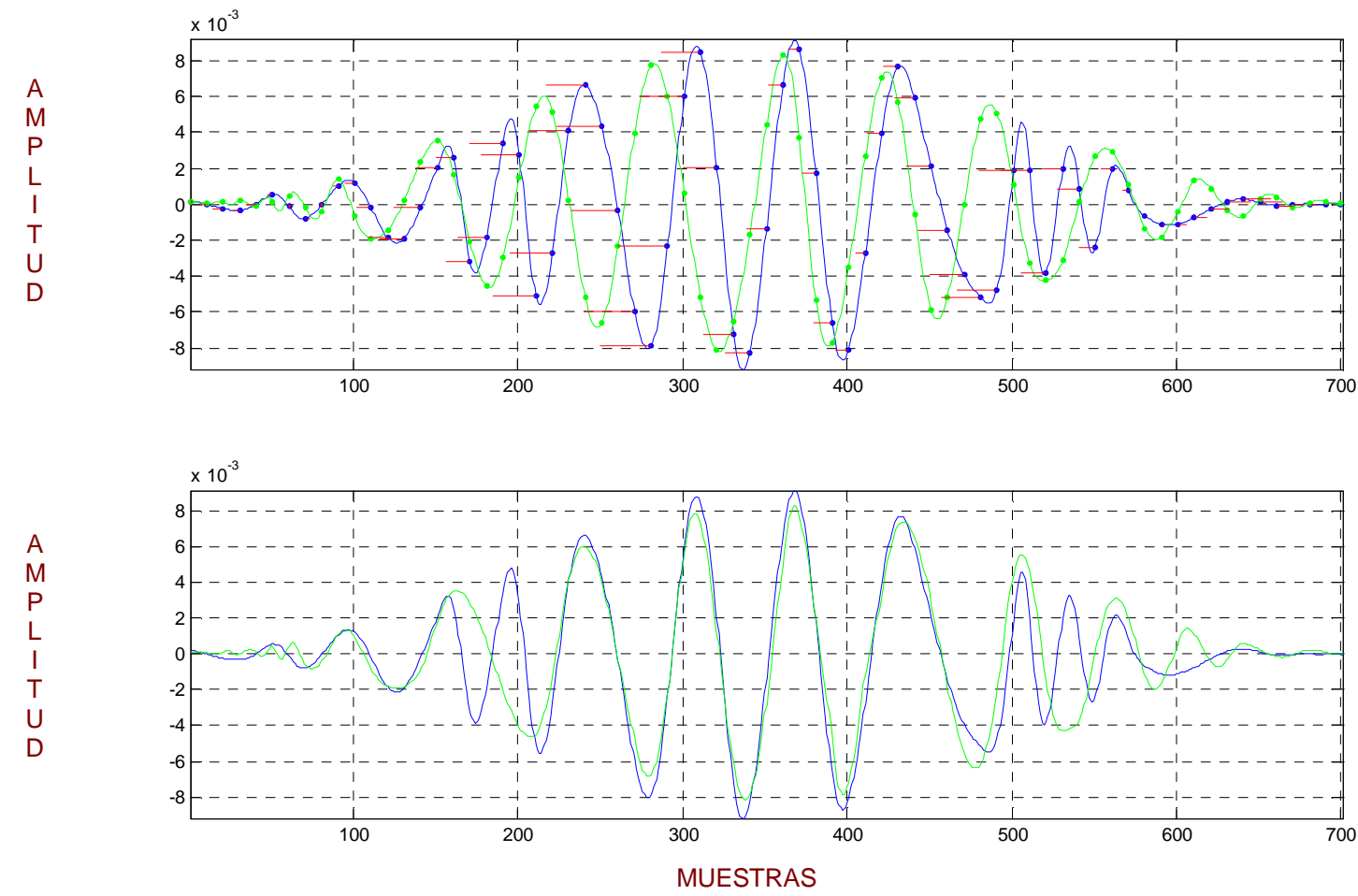

Figura 4.8 En color oscuro se muestra la señal de referencia y en color gris se muestra la señal que es deformada para mejorar la alineación. En la gráfica superior se observan ambas señales antes de aplicar el algoritmo COW mientras que en la inferior se observa ambas señales alineadas.

2. Señal alineada. Se genera la señal de referencia $u$ colocando el segmento seleccionado en apnea en las posiciones indicadas por los valores máximos de la función de correlación. Sin embargo, en este caso el segmento de apnea se procesa previamente por el algoritmo de TW vía Correlation Optimized Warping (COW) [23]. Cuando se aplica TW, el segmento seleccionado es alineado mediante su deformación, acortando o expandiendo el eje de tiempo para hacerlo corresponder con la señal de interferencia (ver Figura 4.8). Con dicho procedimiento se obtiene la señal de referencia denominada SR2. 


\subsubsection{Esquema de Eliminación de Interferencias por Filtrado Adaptativo.}

La aplicación del algoritmo LMS ajusta los coeficientes de la respuesta a impulso del filtro adaptativo, minimizando el error cuadrático medio entre la señal de referencia $u$ y la señal de interferencia [24]. Las señales de referencia SR1 y SR2 serán filtradas cada caso por separado como se describe a continuación:

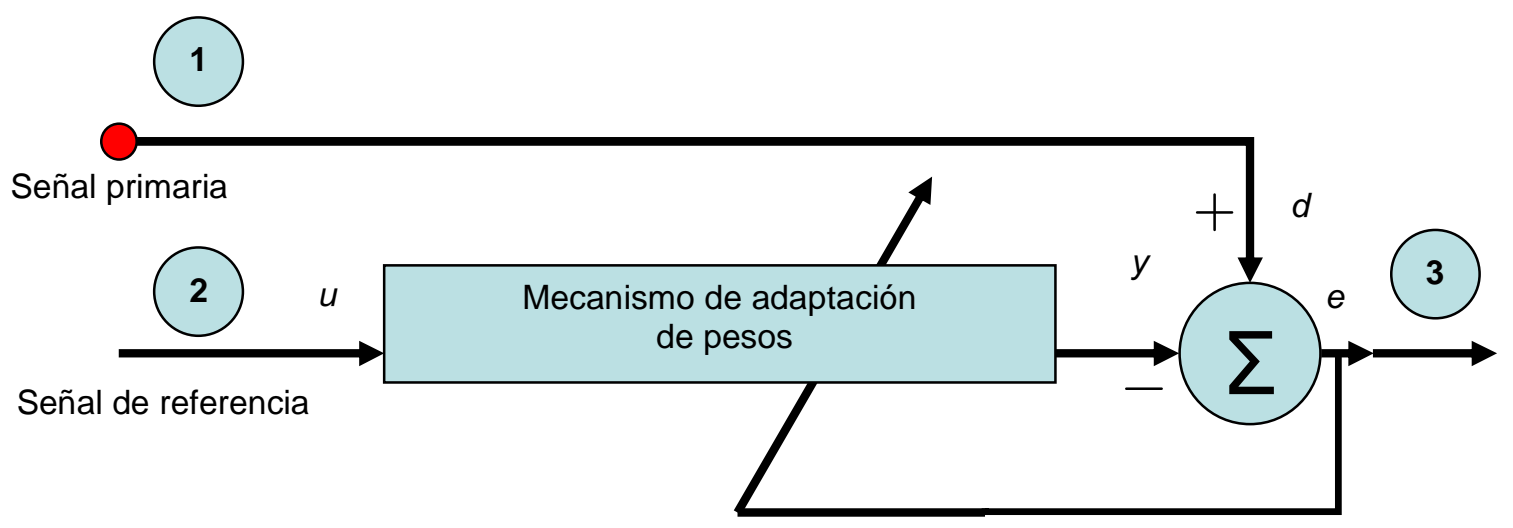

Figura 4.9 Esquema de eliminación de interferencias por filtrado adaptativo.

Obsérvese la Figura 4.9, en la cual se esquematiza el uso del filtrado adaptativo. En el punto 1 donde especifica la señal primaria se introduce el modo de oscilación que contiene la señal de interferencia cardiaca. En el punto 2 se introduce la señal de referencia, SR1 o SR2, mientras que el modo de oscilación filtrado se obtiene del punto número 3 y en la posición denotada por "y" se obtienen los segmentos cuasi-periodicos estimados por el filtro adaptativo.

El filtrado no se aplica a los modos de oscilación en los cuales no se observan componentes cuasi-periódicos, pero en caso de que estos modos contengan información significativa correspondiente al sonido respiratorio serán considerados para la reconstrucción de las señales filtradas.

\subsubsection{Reconstrucción de las Señales Filtradas.}

Se reconstruyen dos señales filtradas, una de ellas utilizando SR1 como señal de referencia y la segunda, utilizando la señal SR2. El procedimiento es como sigue:

a) Se preservan los IMFs correspondientes a escalas cortas de tiempo que presentan una correlación con el flujo respiratorio.

b) Se incluyen los IMFs de escalas largas de tiempo que no hayan sido filtrados pero que contiene componentes asociados al SR. 
c) Se eliminan IMFs de escalas largas de tiempo que presentan una amplitud pequeña, que no contengan información significativa.

La señales filtradas se reconstruyen para IMFs cuyas señales de referencia son procesados sin y con TW. Se suman todos los IMFs procesados y los considerados en los casos (a) y (b).

\subsection{Simulación de Escenarios de Prueba.}

El propósito de simular una señal acústica respiratoria proveniente del tórax es obtener una señal en condiciones controladas para evaluar los alcances y las limitaciones de la metodología propuesta. Las condiciones controladas en esta tesis son: la elección de los ruidos cardiacos a insertar y la selección de su posición; y, la relación señal a ruido, es decir, ruido cardiaco a sonido respiratorio.

De acuerdo al conocimiento de las señales adquiridas reales, se definen las siguientes señales relevantes en la simulación:

- Ruido de fondo.

- Sonidos respiratorios

- Flujo respiratorio.

- Ruido Cardiaco

Las condiciones para la construcción de la señal son las siguientes:

- Se simula una etapa de apnea y una etapa de respiración.

- Se seleccionan dos primeros ruidos cardiacos de la información acústica adquirida de un sujeto sano y uno de ellos se coloca en posiciones conocidas en etapa de apnea y de respiración variando su amplitud para simular ruidos. Lo anterior se realizó con el propósito de simular un posible cambio morfológico de los ruidos cardiacos con la respiración (Figura 4.10 y Figura 4.11).

- Un segundo ruido cardiaco se inserta en posiciones conocidas en ambas zonas.

- Se construyen dos señales respiratorias simuladas considerando relaciones señal cardiaca a ruido de fondo de 50 y $20 \mathrm{~dB}$, respectivamente.

- Para construir la señal de ruido de fondo se genera una señal de ruido aleatorio (distribución gaussiana, $\mu=0$, y $\sigma^{2}=1$ ), con una extensión de 36000 muestras (que corresponde a 7.2 segundos). 


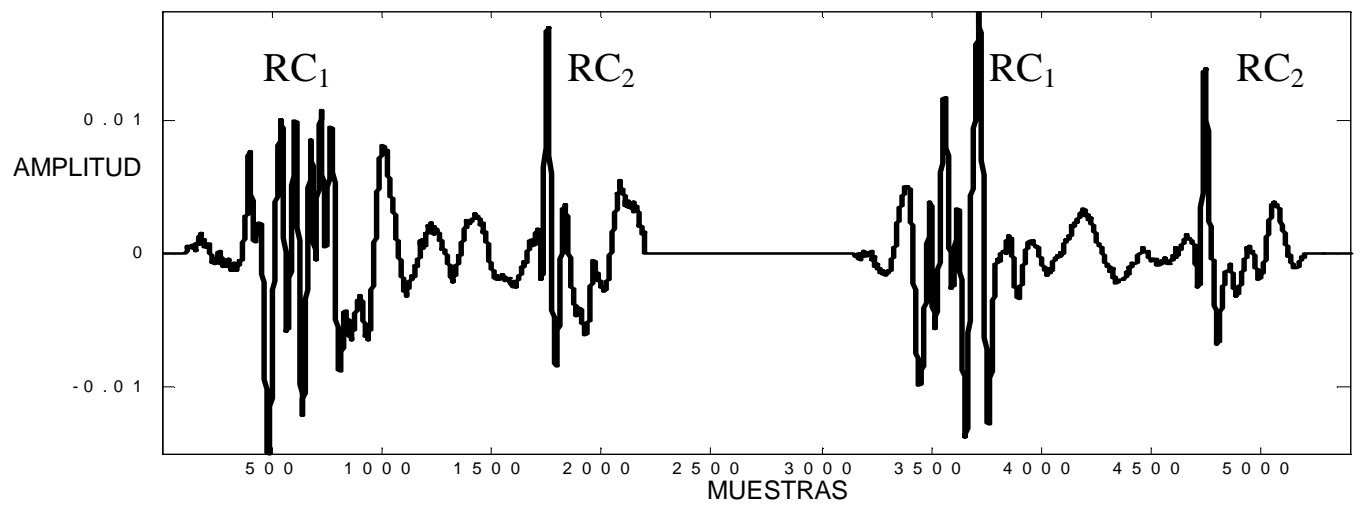

Figura 4.10 Morfología de los ruidos cardiacos involucrados en la señal simulada.

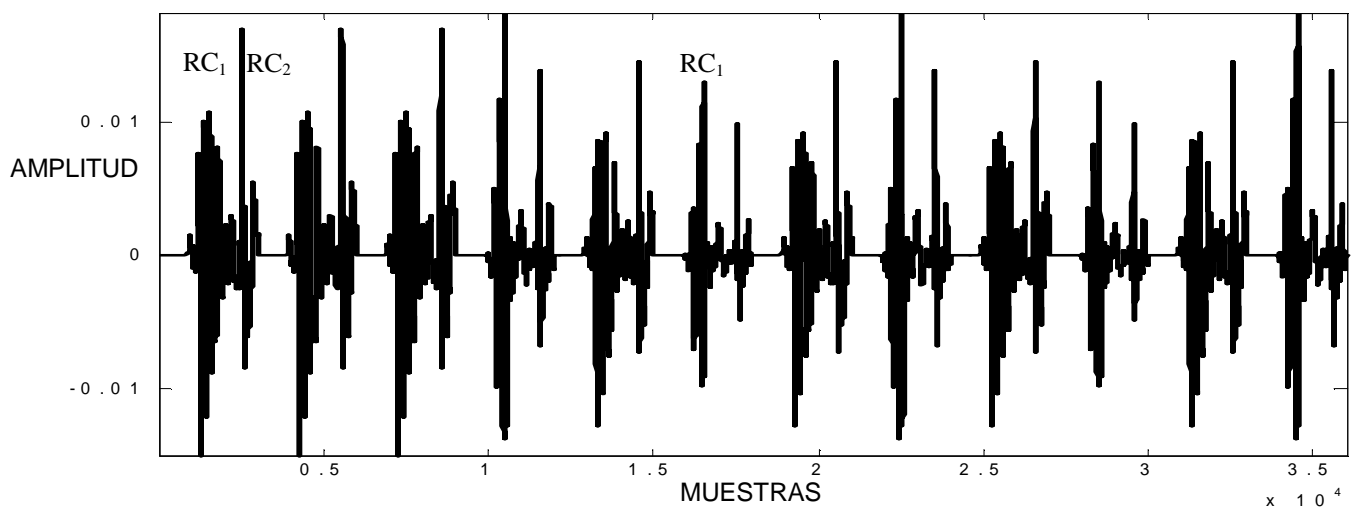

Figura 4.11 Señal de ruidos cardiacos en posiciones conocidas. Nótese el cambio de morfología de los primeros ruidos cardiacos a lo largo de la señal.

- Para la construcción de la señal de sonido respiratorio, se contempla dos etapas, una etapa de apnea y una etapa de respiración. La etapa de apnea se simula considerando únicamente el ruido de fondo y los ruidos cardiacos en posiciones diferentes mientras que la etapa de respiración se simula con una señal aleatoria (distribución gaussiana $\mu=0$, y $\sigma^{2}=1$ ) que se procesa con un filtro FIR pasa bajas de orden 201 y frecuencia de corte de $300 \mathrm{~Hz}$. Para simular la variación en amplitud del sonido respiratorio con el flujo respiratorio, se multiplica la señal filtrada anterior por una señal construida con ventanas intercaladas tipo Hamming, de dos diferentes amplitudes [20]. El sonido inspiratorio requiere de una ventana con mayor amplitud que la correspondiente al sonido espiratorio si se requiere simular una señal adquirida a nivel de la superficie torácica (Figura 4.12). 


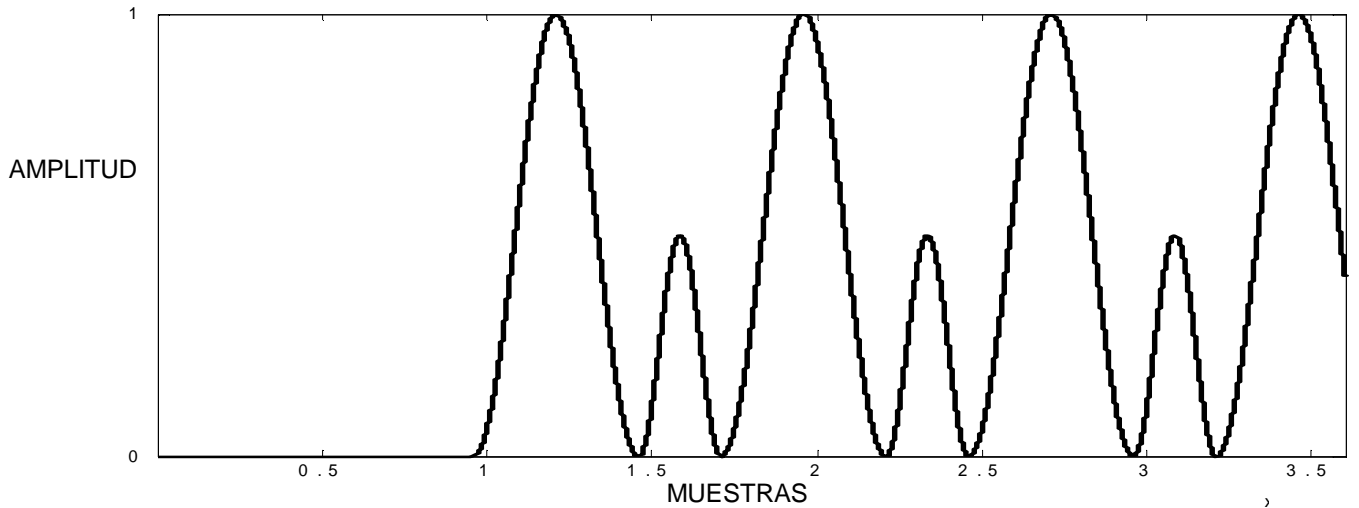

Figura 4.12 Ventanas de Hamming para definir las fases de inspiración y espiración de la señal simulada.

- Una vez construida cada una de las señales anteriormente descritas, todas las señales se suman para obtener una señal resultante (Figura 4.13).

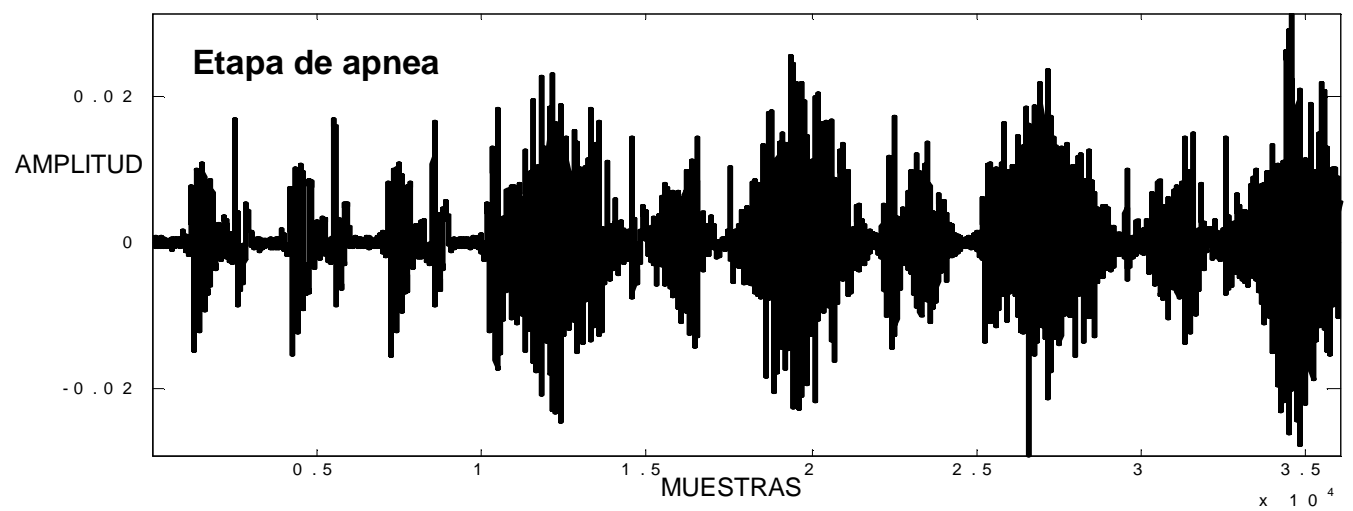

Figura 4.13 Señal respiratoria simulada incluyendo ruidos cardiacos, ruido de fondo y sonido respiratorio con tres ciclos respiratorios completos y una etapa de apnea inicial.

Finalmente, la ecuación matemática que describe la señal simulada se expresa como:

$z(n)=\sum_{i=1}^{M 1} s_{1}\left(n-\Delta_{1 i}\right)+\sum_{i=1}^{M_{2}} s_{2}\left(n-\Delta_{2 i}\right)+\sum_{i=1}^{M 1} s_{1}{ }^{b s}\left(n-\Delta_{1 i}{ }^{b s}\right)+\sum_{i=1}^{4} v\left(n-\Delta_{b i}\right)+w(n)$

donde $z(n)$ representa la señal respiratoria adquirida en un canal determinado,

$S_{1}$ y $S_{2}$ indican el primer y segundo ruido cardiaco respectivamente, en posiciones temporales $\Delta_{1 i}$ y $\Delta_{2 i}$, simulando una frecuencia cardiaca de 100 L/seg. La señal $s_{1}{ }^{b s}$ corresponde al primer ruido cardiaco, modificado por la respiración, resultado de la convolución $s_{1}{ }^{b s}(n)=h_{1 i r} * s_{1}(n)$, donde $h_{1 i r}$ es igual 
a la respuesta al impulso desconocida representando la correlación entre la referencia y la interferencia. El sonido respiratorio es representada por $v(n)$ y $w(n)$ es ruido aditivo de fondo [25].

\subsection{Adquisición Multicanal de Información Acústica en Sujetos Sanos.}

La adquisición multicanal simultánea es una técnica recientemente impulsada para el estudio de los sonidos respiratorios ya que permite la adquisición de señales acústicas en diferentes puntos de la superficie del tórax obteniendo información relevante del estado de los pulmones desde los ápex hasta las bases [26].

La adquisición de la información acústica del tórax en esta tesis se realizó con transductores acústicos modelo BT-1834, insertados en una campana de dimensiones conocidas [13], conectados a un sistema de adquisición de datos por medio de una tarjeta de conversión A/D comercial, a una frecuencia de muestreo de $5000 \mathrm{~Hz}$. Los puntos de adquisición se esquematizan en la Figura 4.14 y son:

IZQ1 corresponde a la línea media axilar izquierda, porción superior.

IZQ2 corresponde a la línea media axilar izquierda, porción media.

IZQ3 corresponde a la línea media axilar izquierda, porción inferior.

DER1 corresponde a la línea media axilar derecha, porción superior.

DER2 corresponde a la línea media axilar derecha, porción media.

DER3 corresponde a la línea media axilar derecha, porción inferior.

PUL corresponde a la zona donde se ausculta por convención el sonido proveniente de la válvula pulmonar.

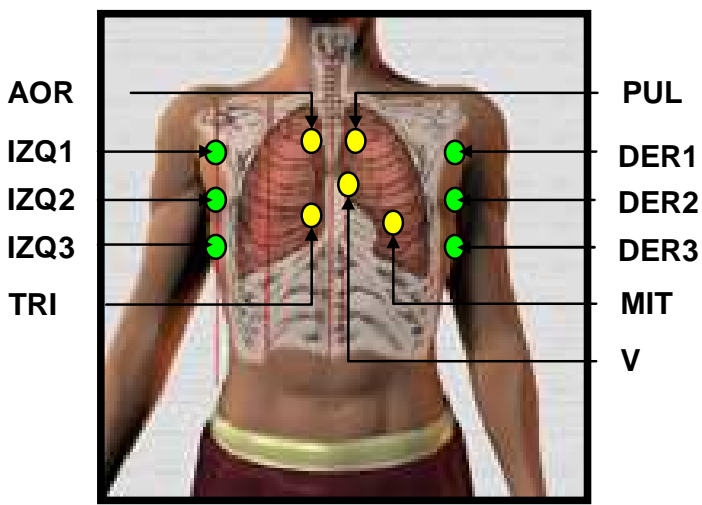

Figura 4.14 Posición de micrófonos para la adquisición de señales acústicas reales. 
AOR corresponde a la zona donde se ausculta por convención el sonido proveniente de la válvula aórtica.

MIT corresponde a la zona donde se ausculta por convención el sonido proveniente de la válvula mitral.

TRI corresponde a la zona donde se ausculta por convención el sonido proveniente de la válvula tricúspide

V corresponde a un canal que captura información proveniente de las cuatro válvulas cardiacas y por tanto se denomina promedio precordial. La idea es capturar información equivalente en magnitud de todos los puntos específicos de captura de los ruidos cardiacos.

Los puntos de adquisición anteriores se seleccionaron con el propósito de hacer variar la influencia del sonido respiratorio y los ruidos cardiacos en términos de su relación de amplitud. En los puntos donde se auscultan los ruidos de las diferentes válvulas cardiacas su intensidad es relevante, y en puntos alejados al corazón, la intensidad de los sonidos respiratorios normalmente se incrementa.

Se adquirieron señales en 11 sujetos masculinos sanos, de complexión delgada y robusta, de edades entre 24 y 46 años, 3 en las instalaciones de la Universidad Autónoma Metropolitana, unidad Iztapalapa, y 8 en el Instituto Nacional de Enfermedades Respiratorias en donde fue posible adquirir también la señal de ECG derivación II por medio de un electrocardiógrafo marca Burdick modelo Eclipse. Ell diagnóstico para definir si un sujeto es sano o no, se determinó con la ayuda de un médico especialista. La Figura 4.15 muestra un ejemplo de las señales adquiridas.

Se calibró la escala de un osciloscopio, por medio de un flujómetro, a flujos de $0.5,1.0,1.5$ y $2.0 \mathrm{~L} / \mathrm{seg}$, y se indicó al sujeto el flujo respiratorio que debe realizar en cada maniobra. Nuevamente, el variar el flujo respiratorio permite manejar la relación de amplitudes, y por lo tanto el SNR, entre el sonido respiratorio y el ruido cardiaco. Una vez colocados los transductores acústicos en el tórax del sujeto, se realizaron maniobras de respiración con 5 segundos iniciales de apnea y un tiempo de 10 
segundos para la etapa de respiración, obteniéndose 15 segundos en total por cada flujo respiratorio.

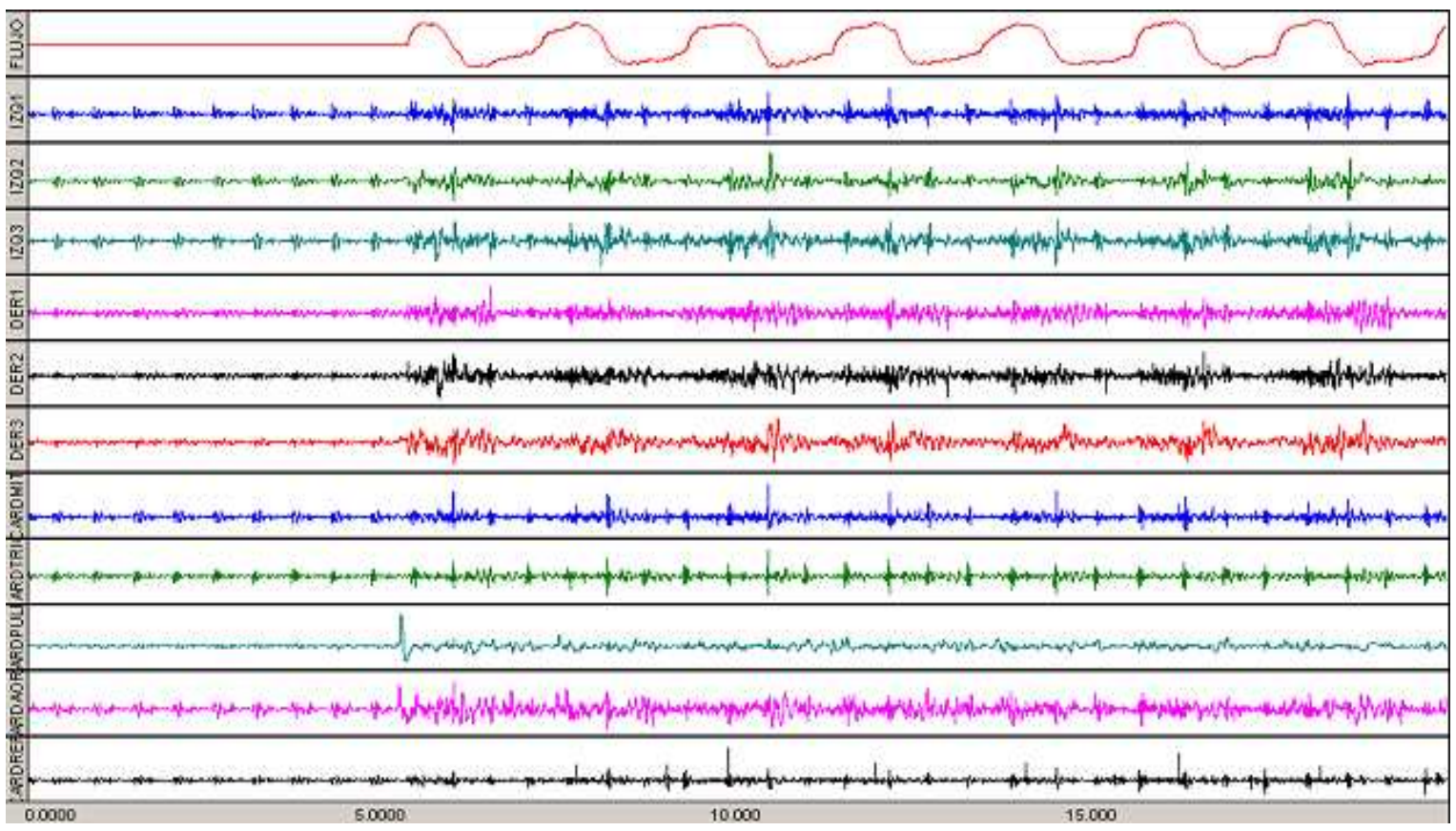

Figura 4.15 (Aquí se reproduce la Figura 2.1).Señales acústicas adquiridas. En la parte superior se muestra el flujo respiratorio (2L/seg), IZQ1 a IZQ3 corresponden a las señales de la línea axilar izquierda, DER1 a DER 3 corresponden a las señales de la línea derecha axilar, y las cinco últimas corresponden a puntos próximos al corazón.

\subsection{Medidas de Desempeño del Esquema Propuesto.}

Para estimar el desempeño de la metodología propuesta se calcularon valores de potencia espectral con base en el espectrograma por transformada de Fourier de tiempo corto. Los elementos importantes a evaluar correspondieron a:

a) En el caso de señales simuladas:

1. Potencia de la señal de interferencia estimada, ruido cardiaco, por el esquema propuesto en el dominio original de la señal simulada, involucrando y sin involucrar TW. Las bandas de interés son de 0 a $300 \mathrm{~Hz}$, de 300 a $600 \mathrm{~Hz}$ y de 600 a $1200 \mathrm{~Hz}$

2. Efecto de la relación señal (ruido cardiaco) a ruido (sonido respiratorio) y señal (ruido cardiaco) a ruido de fondo sobre la estimación de la posición del ruido cardiaco y su reducción posterior.

3. Potencia del sonido respiratorio simulado "limpio" en comparación con la potencia del sonido respiratorio filtrado por el esquema propuesto. 
4. Posibles alteraciones del contenido en frecuencia de los sonidos respiratorios.

b) En el caso de las señales reales:

1. Se calculan las potencias en las posiciones en donde se detectó un ruido cardiaco de interferencia, antes y después del filtrado, y en las mismas bandas de frecuencia antes mencionadas.

2. Para evaluar el desempeño de la metodología, se consideró como una medida de referencia la potencia del ruido cardiaco en apnea para comparar con la potencia del ruido cardiaco en interferencia estimado. Además, se calculó el índice siguiente para todos los sujetos en la base de datos, puntos de adquisición, y flujos.

$$
x=\frac{\mathrm{P}_{\mathrm{RCE}}}{\mathrm{P}_{\text {RCApnea }}} \times 100 \%
$$

donde $\mathrm{P}_{\mathrm{RCE}}$ es la potencia de los ruidos cardiacos de interferencia estimado y $\mathrm{P}_{\mathrm{RCApnea}}$ es la potencia de los ruidos cardiacos localizados en etapa de apnea.

El procedimiento de evaluación incluye:

- Determinación de intervalos de tiempo del ruido cardiaco de interferencia en la señal simulada.

Las potencias de los ruidos cardiacos son obtenidas con los intervalos conocidos que corresponden, como punto de inicio, a las posiciones en donde se insertaron los ruidos cardiacos, y como punto final, a la suma de la posición de inicio y el ancho del ruido cardiaco.

- Determinación de los intervalos de tiempo en señales reales.

Para calcular las potencias de los ruidos cardiacos de interferencia estimados en el dominio original, se utiliza, en el dominio de la descomposición, los máximos de la correlación cruzada entre el segmento seleccionado en apnea y el modo de oscilación correspondiente, estableciendo los intervalos como se muestra en la Tabla 4.1 


\begin{tabular}{|c|c|c|c|c|c|c|c|c|c|}
\hline CANAL & $\begin{array}{c}\text { MODO DE } \\
\text { OSCILACIÓN }\end{array}$ & \multicolumn{1}{|c|}{$\begin{array}{c}\text { INTERVALO DEL } \\
\text { SEGMENTO DE } \\
\text { REFERENCIA }\end{array}$} & \multicolumn{7}{|c|}{ MÁXIMOS } \\
\hline 8 & 5 & 6818 & 7229 & 539.89 & 6817 & 12851 & 24551 & 29890 & 0 \\
\hline 8 & 6 & 6719 & 7412 & 542.33 & 6718.3 & 12724 & 18772 & 24424 & 29736 \\
\hline 8 & 7 & 6604 & 7557 & 412.49 & 6603.6 & 12722 & 18629 & 24308 & 29643 \\
\hline 8 & 8 & 6369 & 8341 & 6369.7 & 12435 & 18477 & 24166 & 29328 & 0 \\
\hline 8 & 9 & 114 & 3715 & 0 & 0 & 0 & 0 & 0 & 0 \\
\hline
\end{tabular}

Tabla 4.1 Selección de la señal de referencia en los intervalos indicados y sus máximos correspondientes por modo de oscilación en la señal de sonido respiratorio.

1. Se calculan los intervalos para cada IMF procesado. Para explicar el procedimiento se hará referencia a los datos de la tabla 4.1:

La primera y segunda columna de la tabla 4.1, indica el canal adquirido y el modo de oscilación que fueron procesados por la metodología propuesta, respectivamente. Las dos siguientes columnas corresponden al intervalo, en muestras, del segmento de información cardiaca en apnea seleccionado en cada IMF. En las columnas siguientes se encuentran los máximos obtenidos de la correlación cruzada entre el segmento seleccionado y el resto del IMF. Las celdas con valores cero indican que no fue localizado algún ruido cardiaco de interferencia, sin embargo, el IMF que se intentó procesar debe de adicionarse para la reconstrucción de la señal resultante, ya que presenta información relevante. Es de hacer notar en la tabla 4.1, los máximos de la función de correlación se encuentran cercanos en valor en los diferentes modos de oscilación. La cuestión es utilizar ahora esta información redundante de posición para establecer los intervalos de tiempo en donde se detectaron los ruidos cardiacos de interferencia en cada IMF procesado para calcular la potencia espectral en el dominio original de la señal.

2. Se ordenan los máximos de la función de correlación por canal en forma ascendente, como se muestra en la tabla 4.2

3. Para la obtención de los intervalos de tiempo del ruido cardiaco de interferencia detectado, se utiliza el siguiente procedimiento:

a. Se inicia con el primer elemento del arreglo, es decir, el elemento de menor valor, el número 412.49 en la tabla 4.2. 


\begin{tabular}{|c|c|c|c|c|c|c|c|c|c|c|c|}
\hline & \multicolumn{10}{|c|}{ NÚMERO MÁXIMO } \\
\hline & 1 & 2 & 3 & 4 & 5 & 6 & 7 & 8 & 9 & 10 & 11 \\
\hline $\begin{array}{c}\text { POSICIÓN } \\
\text { CORRESPONDIENTE }\end{array}$ & 412.9 & 539.9 & 542.3 & 6369.7 & 6603.6 & 6718.3 & 6817 & 12435 & 12722 & 12724 & 12851 \\
\hline & 12 & 13 & 14 & 15 & 16 & 17 & 18 & 19 & 20 & 21 & 22 \\
\hline $\begin{array}{c}\text { POSICIÓN } \\
\text { CORRESPONDIENTE }\end{array}$ & 18477 & 18629 & 18772 & 24166 & 24308 & 24424 & 24551 & 29328 & 29643 & 29736 & 29890 \\
\hline
\end{tabular}

Tabla 4.2 Ejemplo de la posición correspondiente al máximo de la función de autocorrelación por canal.

b. Se calcula el extremo superior con la longitud del segmento de la señal de referencia respectiva, es decir, el intervalo correspondiente es $(6604,7557)$. El extremo superior se obtiene con la ecuación $412.49+7557-6604$, y por lo tanto el intervalo para el cálculo de la potencia del ruido cardiaco de interferencia detectado es $(412,49,1365.49)$.

1. Si el siguiente máximo, el número 539.89, se encuentra en el intervalo anteriormente estimado, se calcula nuevamente el extremo superior utilizando el intervalo del segmento de referencia que corresponde al número y se elige el valor mayor.

2. Si el siguiente máximo, 6369.7, no se encuentra en el intervalo anteriormente estimado, éste se selecciona como el próximo extremo inferior de un nuevo intervalo y se sigue el paso b hasta terminar con todos los máximos. 


\section{CAPÍTULO 5 RESULTADOS}

\subsection{Información Acústica Presente en las Señales Multicanal Adquiridas.}

Esta sección se compone de resultados en señales simuladas y señales adquiridas reales. Sin embargo, en favor de la claridad en la exposición, en el caso de datos adquiridos se presentará un número limitado de gráficas y datos estimados, en condiciones de bajo y alto flujo respiratorio, dado que se adquirieron las señales de 11 sujetos. En un apéndice se proporcionan los resultados de todos los datos adquiridos.

La Figura 4.15 es un ejemplo de las señales multicanal adquiridas a un flujo de 2 L/seg. La etapa de apnea contiene varios ruidos cardiacos y en la etapa de respiración se observa un incremento del sonido respiratorio con la fase inspiratoria y espiratoria, indicadas por ondas positivas y negativas en el flujo respiratorio, respectivamente. En la etapa de apnea, los ruidos cardiacos presentan diferentes amplitudes dependiendo de la cercanía o lejanía del corazón. Así, la de mayor amplitud es la señal correspondiente a la posición en donde se ausculta normalmente el ruido producido por la válvula mitral. También se observa que la morfología de los ruidos cardiacos en esta etapa no presenta cambios aparentes, tal y como lo indican los esfuerzos en la referencia 19. En contraste, en la etapa de respiración se observa un cambio en la morfología de los ruidos cardiacos. En las posiciones de registro más alejadas del corazón, la presencia de los ruidos cardiacos se ve completamente disminuida.

\subsection{Modos de Oscilación (IMFs) en Señales Simuladas y Adquiridas Reales.}

\subsubsection{Señales Simuladas.}

\subsubsection{Señales Acústicas Simuladas con Diferente Nivel de Ruido de Fondo.}

En la Figura 5.1 se presenta la señal acústica simulada con una relación señalruido de fondo de $20 \mathrm{~dB}$. Mientras que la Figura 5.2 se presenta la señal acústica simulada con una relación señal-ruido de fondo de $50 \mathrm{~dB}$. El cambio en la SNR de la señal simulada, en particular de la señal cardiaca, con respecto al ruido de fondo se observa en la línea de base en la etapa de apnea al hacerse ésta más gruesa. También obsérvese la morfología de la señal respiratoria simulada. Ésta se advierte muy cercana 
a la morfología de la señal que se puede adquirir en alguna zona en la superficie torácica, es decir información de la fase inspiratoria de mayor magnitud que la información de la fase espiratoria. Su comportamiento en el dominio tiempo-frecuencia se observa en la Figura 5.49 y 5.50.

\subsubsection{IMFS de las Señales Simuladas.}

De la Figura 5.3 a la Figura 5.5 se presenta la descomposición en IMFs de la señal simulada con una relación señal-ruido de fondo de $20 \mathrm{~dB}$ y de la Figura 5.6 a la Figura 5.8 se presenta la descomposición en IMFs de la señal simulada con una relación señal-ruido de fondo de $50 \mathrm{~dB}$. El EMD proporcionó de 14 a 15 IMFs, con los primeros IMFs representando escalas de tiempo cortas, esto es, valores altos de frecuencias instantáneas. En ambos casos, 20 y 50 dB, a partir del IMF3 o IMF4, y hasta el IMF10, aparecen elementos cuasi-periodicos en los modos de oscilación que coinciden temporalmente con los ruidos cardiacos insertados. También se puede observar que el ruido cardiaco y el sonido respiratorio comparten varias escalas de tiempo, impidiendo eliminar simplemente los IMFs correspondientes a ruido cardiaco, de ahí la importancia del filtro adaptativo. A partir del $\mathrm{IMF}_{9}$ en adelante se presentan las escalas de tiempo largas, correspondientes a frecuencias instantáneas bajas.

\subsubsection{Señales Reales.}

Un ejemplo de las señales adquiridas en un sujeto sano se presenta en la Figura 5.9. Las señales acústicas se obtuvieron en zonas donde se auscultan normalmente los ruidos cardiacos a un flujo de $0.5 \mathrm{~L} / \mathrm{seg}$. Las señales acústicas corresponden a la válvula mitral, tricúspide, pulmonar, aórtica y finalmente una señal "promedio" precordial, respectivamente. En la Figura 5.10, se presentan las señales acústicas de la línea media axilar izquierda y en la Figura 5.11, las señales correspondientes a la línea media axilar derecha para el mismo sujeto. De la Figura 5.12 a la Figura 5.14 se presentan señales acústicas correspondientes a flujo de $2.0 \mathrm{~L} / \mathrm{seg}$.

\subsubsection{EMD de Señales Acústicas en Posiciones Cercanas al Corazón.}

De la Figura 5.15 a la 5.17 se presenta la descomposición en IMFs de una señal acústica obtenida de un sujeto sano en la zona donde se ausculta la válvula tricúspide a 
un flujo de $0.5 \mathrm{~L} / \mathrm{seg}$. El EMD proporcionó $15 \mathrm{IMFs}$, con los primeros IMFs representando escalas de tiempo cortos, esto es, valores altos de frecuencias instantáneas. Dado que no se observa ninguna correlación temporal de las magnitudes de $\mathrm{IMF}_{1}$ al $\mathrm{IMF}_{3}$ con el flujo respiratorio, estos modos se pueden eliminar ya que corresponden a ruido de fondo. Para las señales adquiridas en posiciones más cercanas al corazón, y a un flujo respiratorio bajo, los elementos cuasi-periódicos relacionados con los ruidos cardiacos son más evidentes en todos los modos de oscilación restantes. Conforme el flujo respiratorio se incrementa, aparecen más IMFs con las escalas de tiempo asociadas a sonido respiratorio.

\subsubsection{EMD de Señales Acústicas en Posiciones Alejadas del Corazón.}

En las Figura 5.18 a la 5.20 se presenta la descomposición en IMFs de una señal adquirida en una posición lejana al corazón, en la línea axilar izquierda media a flujo de 0.5L/seg. En las Figura 5.21 a la 5.23 se presenta la descomposición en IMFs de una de señal adquirida en una posición lejana al corazón en la línea axilar derecha media a flujo de $0.5 \mathrm{~L} / \mathrm{seg}$. En la Figura 5.24 a la 5.26 se presenta la descomposición en IMFs de una señal adquirida en una posición lejana al corazón en la línea axilar izquierda basal a un flujo de $2 \mathrm{~L} / \mathrm{seg}$.

\subsubsection{Señales Simuladas Filtradas con TW y sin TW.}

En las Figura 5.27 a la 5.28 se presentan las señales resultantes después de ser procesadas con la metodología anteriormente descrita. Se muestra la señal original, la señal filtrada sin utilizar algoritmo de alineación y utilizando algoritmo de alineación para señales simuladas con $20 \mathrm{~dB}$ y $50 \mathrm{~dB}$.

\subsubsection{Señales Reales Filtradas con TW y sin TW.}

En las Figura 5.29 a la 5.49 se presenta las señales resultantes después de ser procesadas con la metodología anteriormente descrita. Se muestra la señal original, la señal filtrada sin utilizar algoritmo de alineación y utilizando algoritmo de alineación para todos los casos señales cercanas al corazón, de la línea axilar izquierda y de la línea axilar derecha para un flujos de $0.5 \mathrm{~L} / \mathrm{seg}$ y $2 \mathrm{~L} / \mathrm{seg}$. En algunas gráficas se incluyen 
flechas que indican posiciones de ruidos cardiacos antes y después de aplicar la metodología propuesta para observar el efecto obtenido.

\subsection{Medidas de Desempeño en Escenarios de Prueba.}

En la Tabla 5. 1 y Tabla 5. 2 se presentan las potencias espectrales calculadas para las señales simuladas de $20 \mathrm{~dB}$ y $50 \mathrm{~dB}$ respectivamente, en las bandas de frecuencias de 0 a 300, de 300 a 600 y de 600 a $1200 \mathrm{~Hz}$ para tres primeros ruidos cardiacos insertados en la etapa de apnea y 9 insertados en la etapa de respiración. Las tablas presentan los datos siguientes:

- Número de ruido cardiaco insertado en la etapa de apnea o de respiración.

- Relación señal (ruido cardiaco) a ruido (sonido respiratorio) para cada ruido cardiaco insertado.

- Potencias de ruidos cardiacos insertados en el dominio original de la señal.

- Potencias de ruidos cardiacos más ruido de fondo.

- Potencias de ruido cardiaco de interferencia estimado por la metodología sin aplicar TW (Obtenida a partir de la suma total de la señal de salida y del filtro adaptativo, de cada uno de los IMFs procesados).

- Potencias de ruido cardiaco de interferencia estimado por la metodología con TW.

- Potencias del sonido respiratorio simulado "limpio".

- Potencias del sonido respiratorio más ruido de fondo.

- Potencias del sonido respiratorio más ruido de fondo y ruidos cardiacos.

- Potencias de la señal de sonido respiratorio filtrado sin TW (Obtenida a partir de la suma total de la señal de error del filtro adaptativo, de cada uno de los IMFs procesados).

- Potencias de la señal de sonido respiratorio filtrado sin TW más los primeros IMFs.

- Potencias de la señal de sonido respiratorio filtrado con TW.

- Potencias de la señal de sonido respiratorio filtrado con TW más los primeros IMFs

\subsection{Medidas de Desempeño en Datos Reales.}

En las tablas Tabla 5. 3.a a la Tabla 5. 3.k se presentan las potencias calculadas para las señales reales adquiridas en diferentes puntos del tórax de un sujeto a un flujo de $0.5 \mathrm{~L} / \mathrm{seg}$. En las Tabla 5. 4.a a Tabla 5. 4.k se presentan las potencias calculadas 
para las señales reales obtenidas en diferentes puntos del tórax de un sujeto a un flujo de $1.0 \mathrm{~L} / \mathrm{seg}$. En las tablas Tabla 5. 5.a a la Tabla 5. 5.k se presentan las potencias calculadas para las señales reales obtenidas en diferentes puntos del tórax de un sujeto a un flujo de $1.5 \mathrm{~L} / \mathrm{seg}$. En las tablas Tabla 5. 6.a a la Tabla 5. 6.k se presentan las potencias calculadas para las señales reales obtenidas en diferentes puntos del tórax de un sujeto a un flujo de $2.0 \mathrm{~L} / \mathrm{seg}$.

Las letras de las tablas corresponden a las señales que se indican:
a. Línea axilar izquierda, región apical
b. Línea axilar izquierda, región media
c. Línea axilar izquierda, región basal
d. Línea axilar derecha, región apical
e. Línea axilar derecha, región media
f. Línea axilar derecha, región basal
g. Zona de auscultación para válvula mitral
h. Zona de auscultación para válvula tricúspide
i. Zona de auscultación para válvula pulmonar
j. Zona de auscultación para válvula aórtico
k. Posición precordial promedio

En las tablas se proporcionan la posición inicial y final estimada para cada ruido cardiaco detectado en la zona de apnea y en la de respiración. Además, se proporcionan las potencias espectrales siguientes:

- Potencia de la señal real adquirida en el dominio original.

- Potencias de la señal de sonido respiratorio filtrado sin TW.

- Potencias de la señal de sonido respiratorio filtrado con TW.

- Potencias de ruido cardiaco de interferencia estimado sin TW.

- Potencias de ruido cardiaco de interferencia estimado con TW.

Se grafican los resultados obtenidos de la ecuación 4.1. Las gráficas 5.51 a la 5.54, se presentan los promedios de las potencias calculadas por flujo, en las zonas donde se adquieren las señales acústicas en el protocolo propuesto por esta tesis, por ancho de banda que va de 0-300, 300-600 y de 600 a $1200 \mathrm{~Hz}$. De igual forma se grafica y se calcula la varianza por flujo, por canal, para todos los sujetos, en diferentes anchos de banda. Por último se calcula el promedio del promedio de las potencias. 


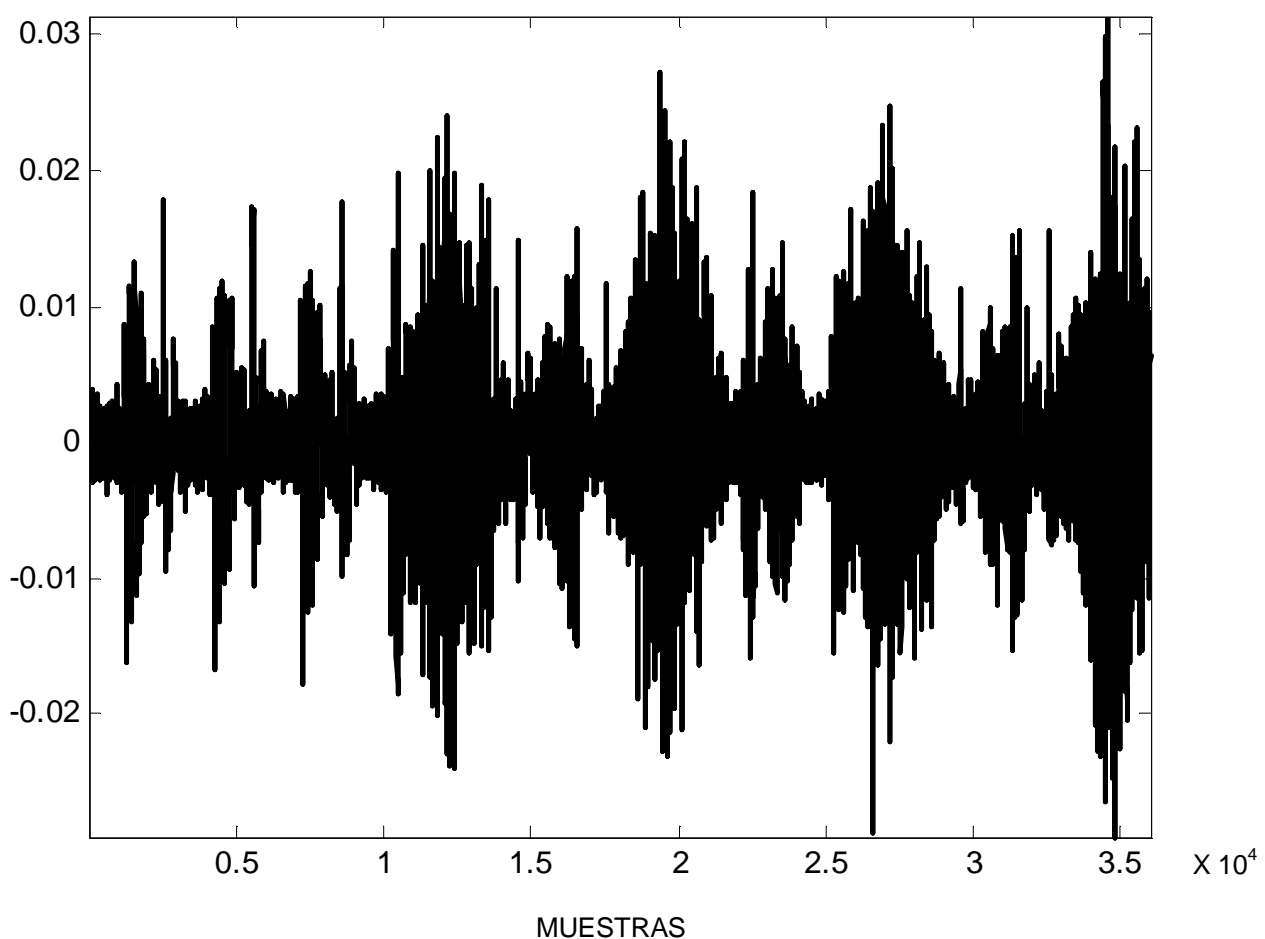

Figura 5.1 Señal acústica simulada con una relación señal-ruido de fondo de $20 \mathrm{~dB}$

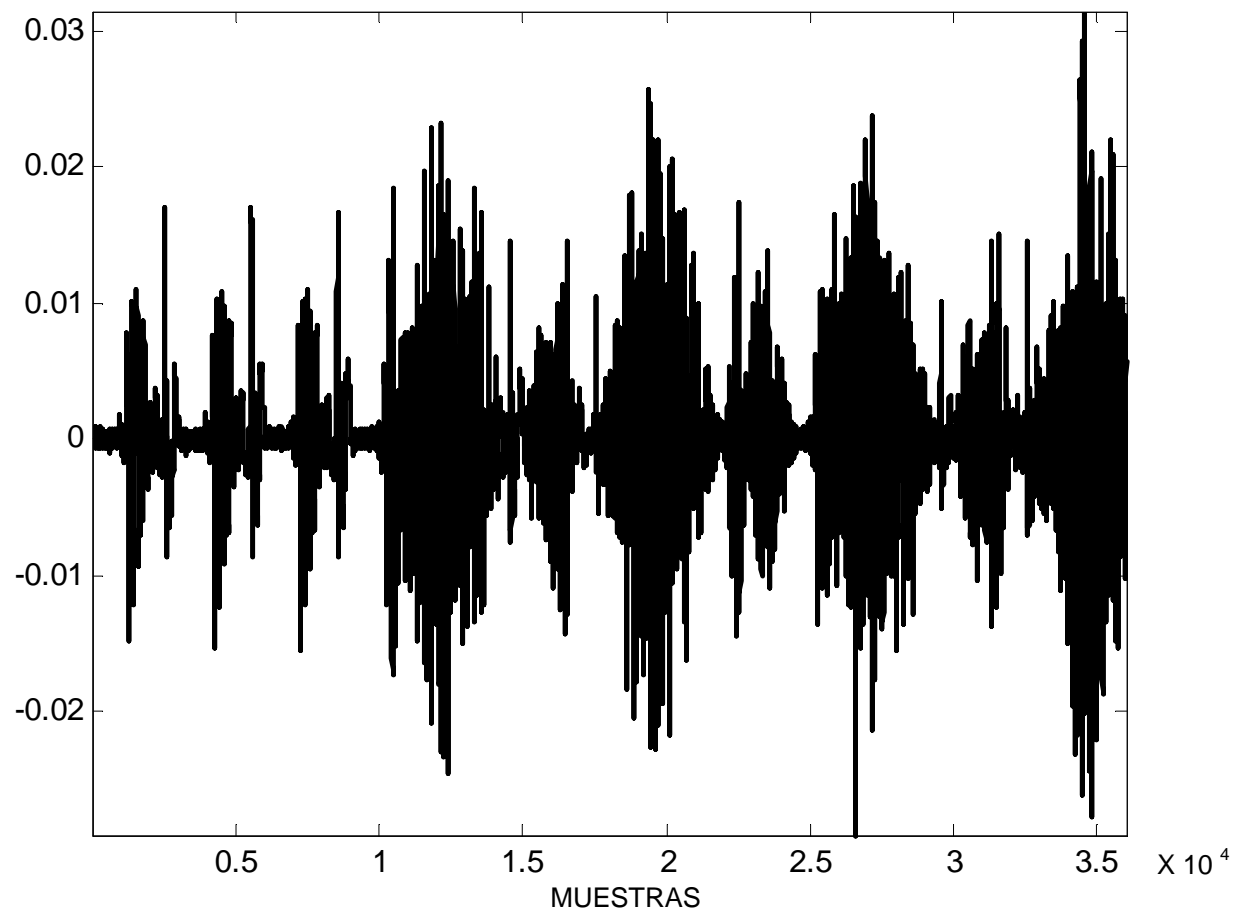

Figura 5.2 Señal acústica simulada con una relación señal-ruido de fondo de $50 \mathrm{~dB}$ 


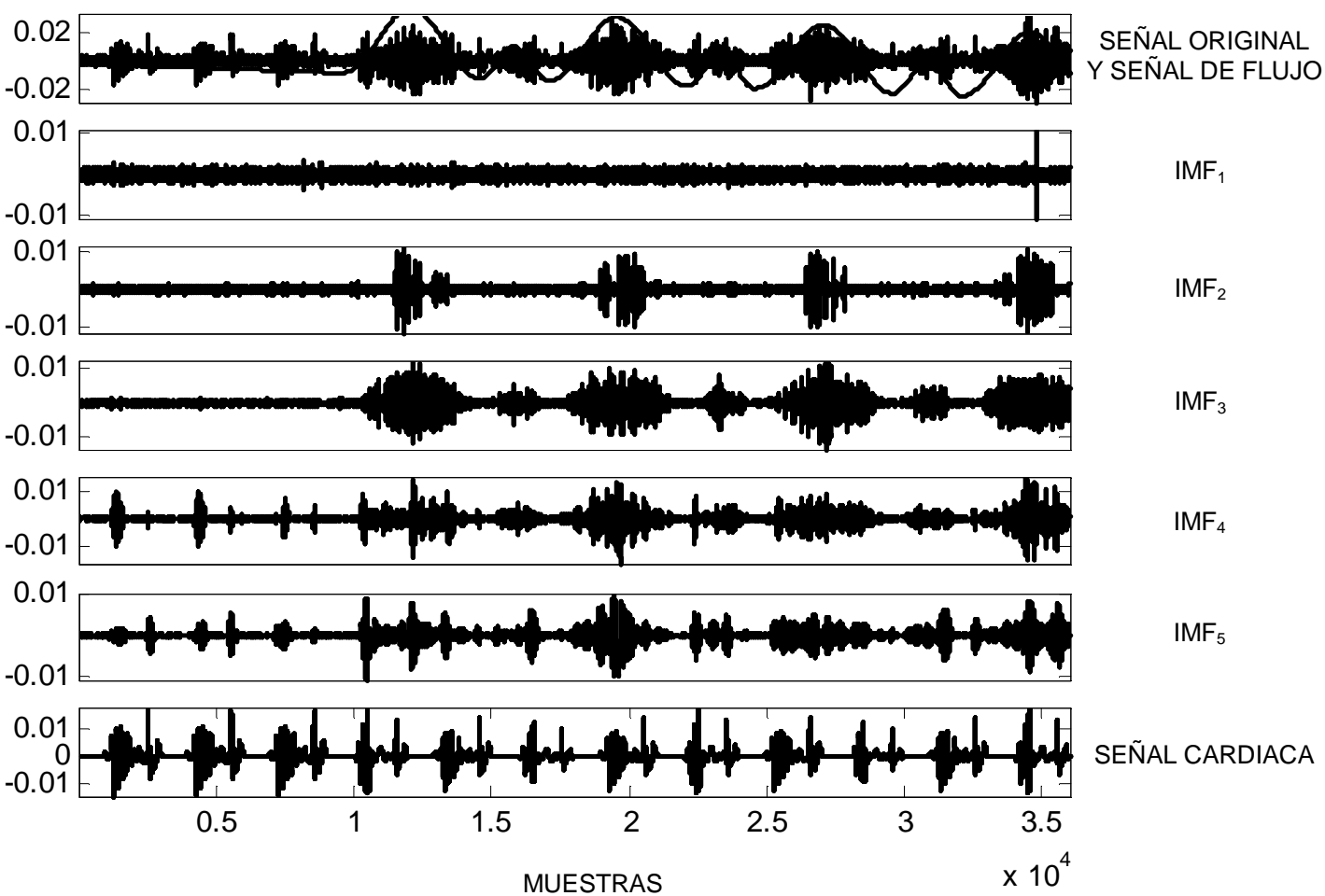

Figura 5.3 IMFs 1 al 5 de la señal acústica simulada con una relación señal-ruido de fondo de $20 \mathrm{~dB}$.

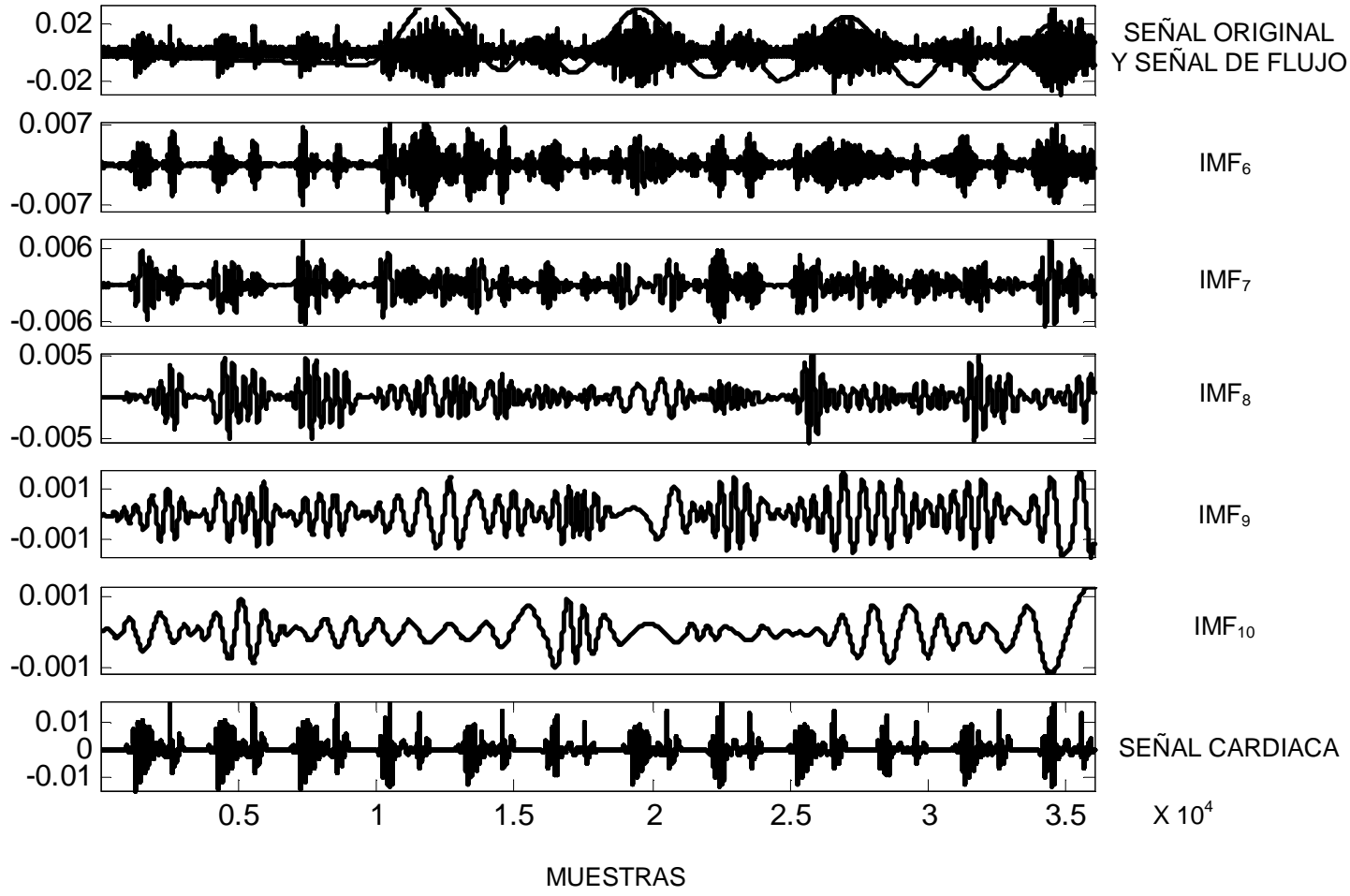

Figura 5.4 IMFs 6 al 10 de la señal acústica simulada con una relación señal-ruido de fondo de 20dB. 


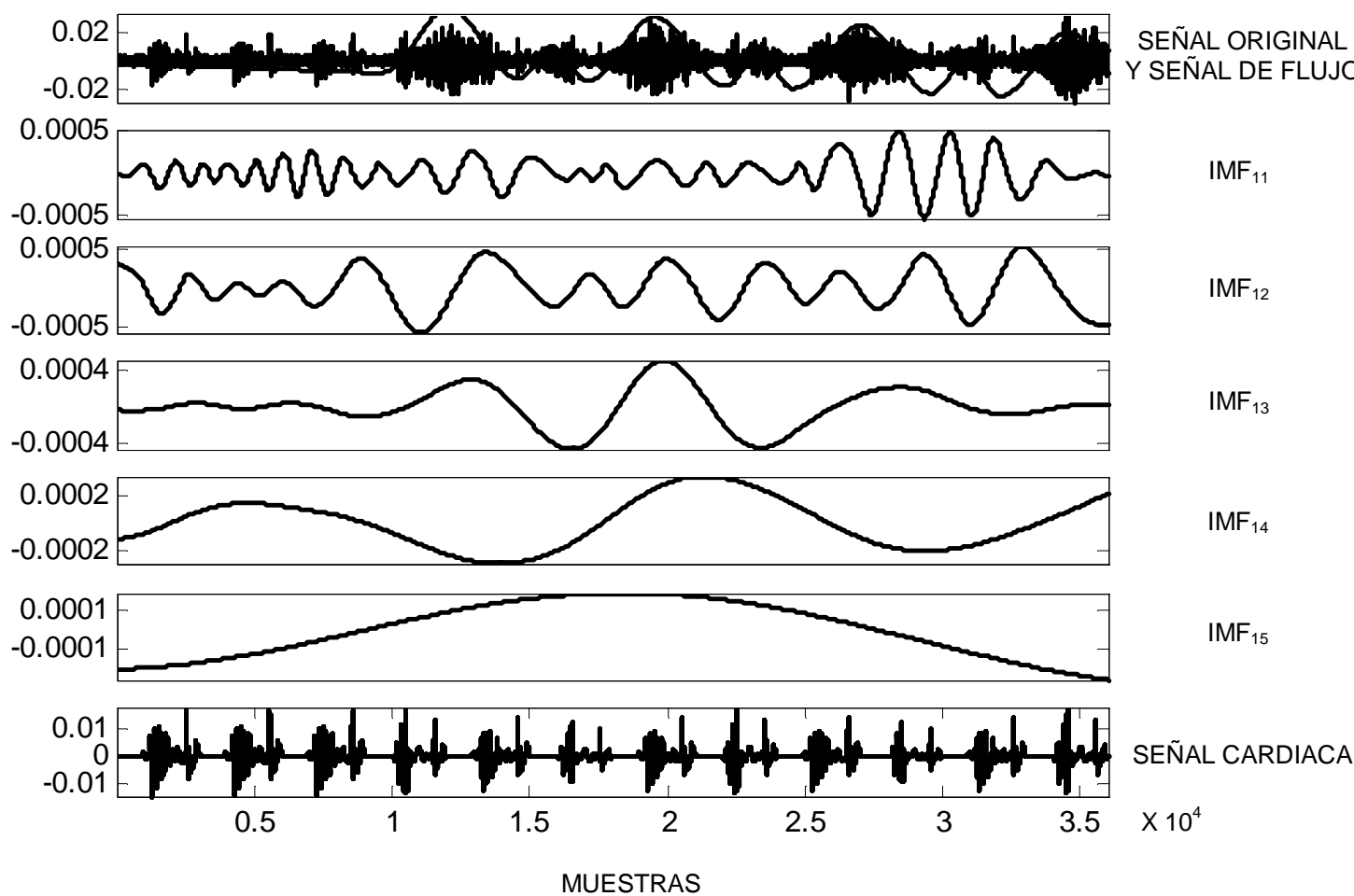

Figura 5.5 IMFs 11 al 15 de la señal acústica simulada con una relación señal-ruido de fondo de $20 \mathrm{~dB}$.

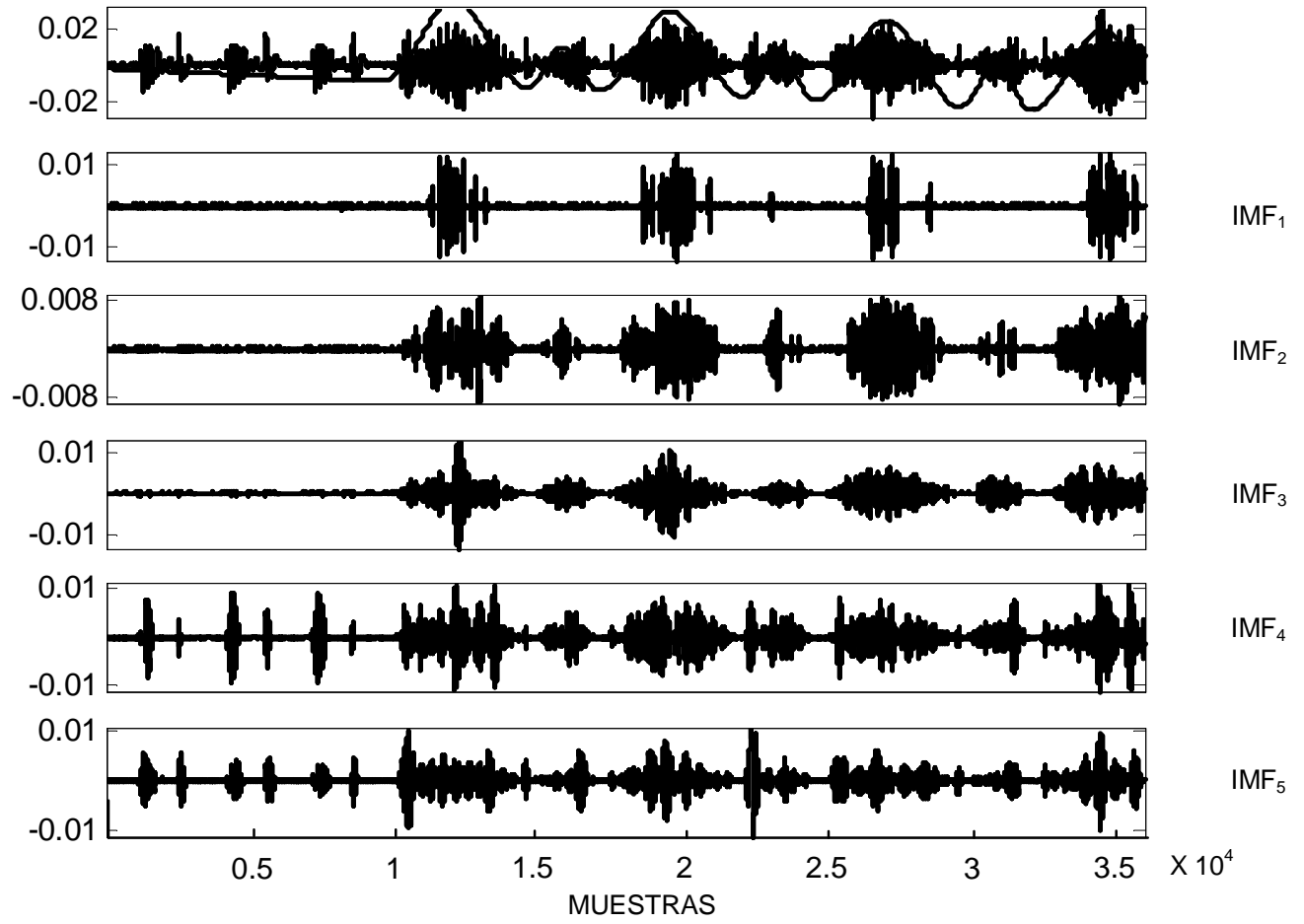

Figura 5.6 IMFs 1 al 5 de la señal acústica simulada con una relación señal-ruido de fondo de $50 \mathrm{db}$. 


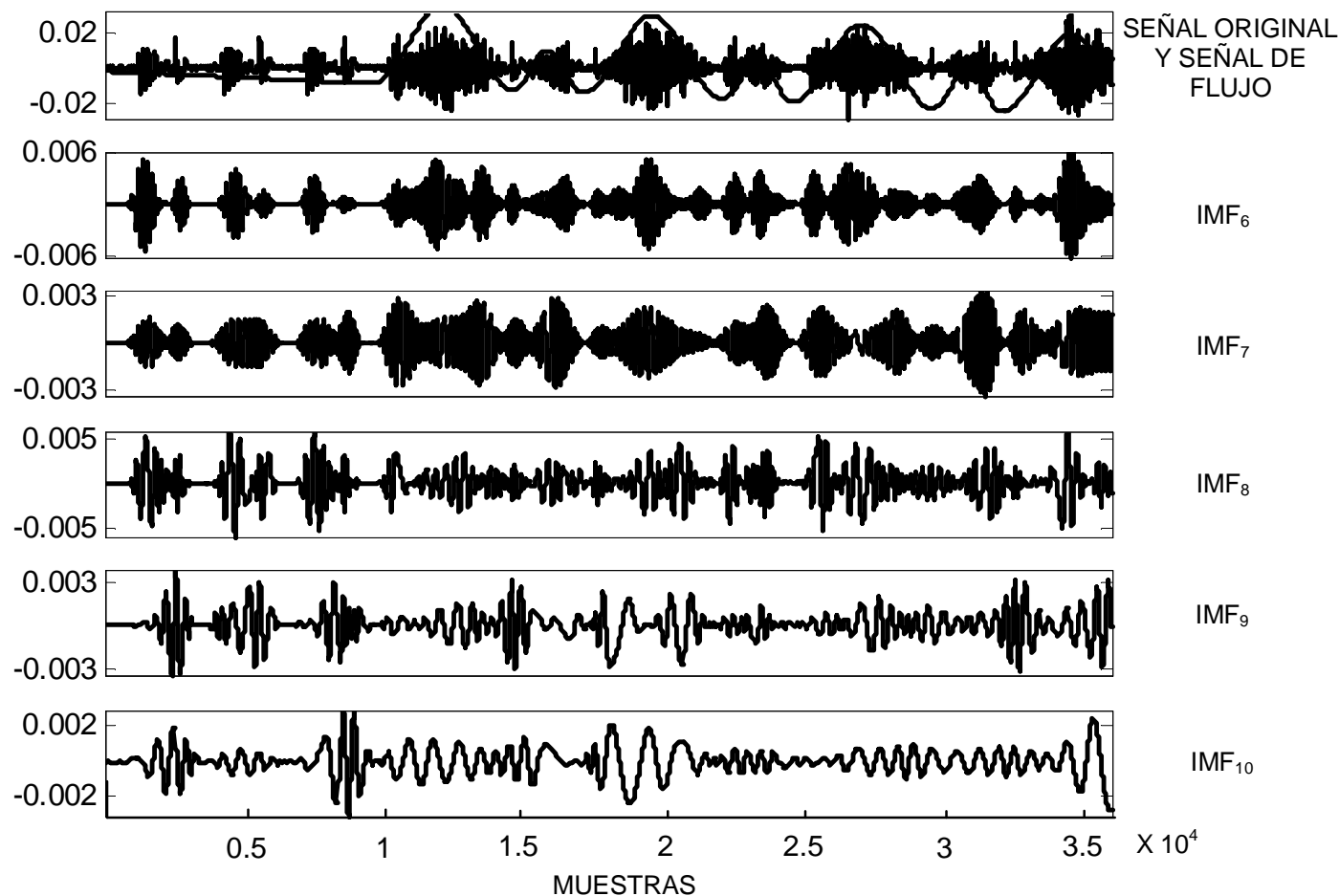

Figura 5.7 IMFs 6 al 10 de la señal acústica simulada con una relación señal-ruido de fondo de 50dB.

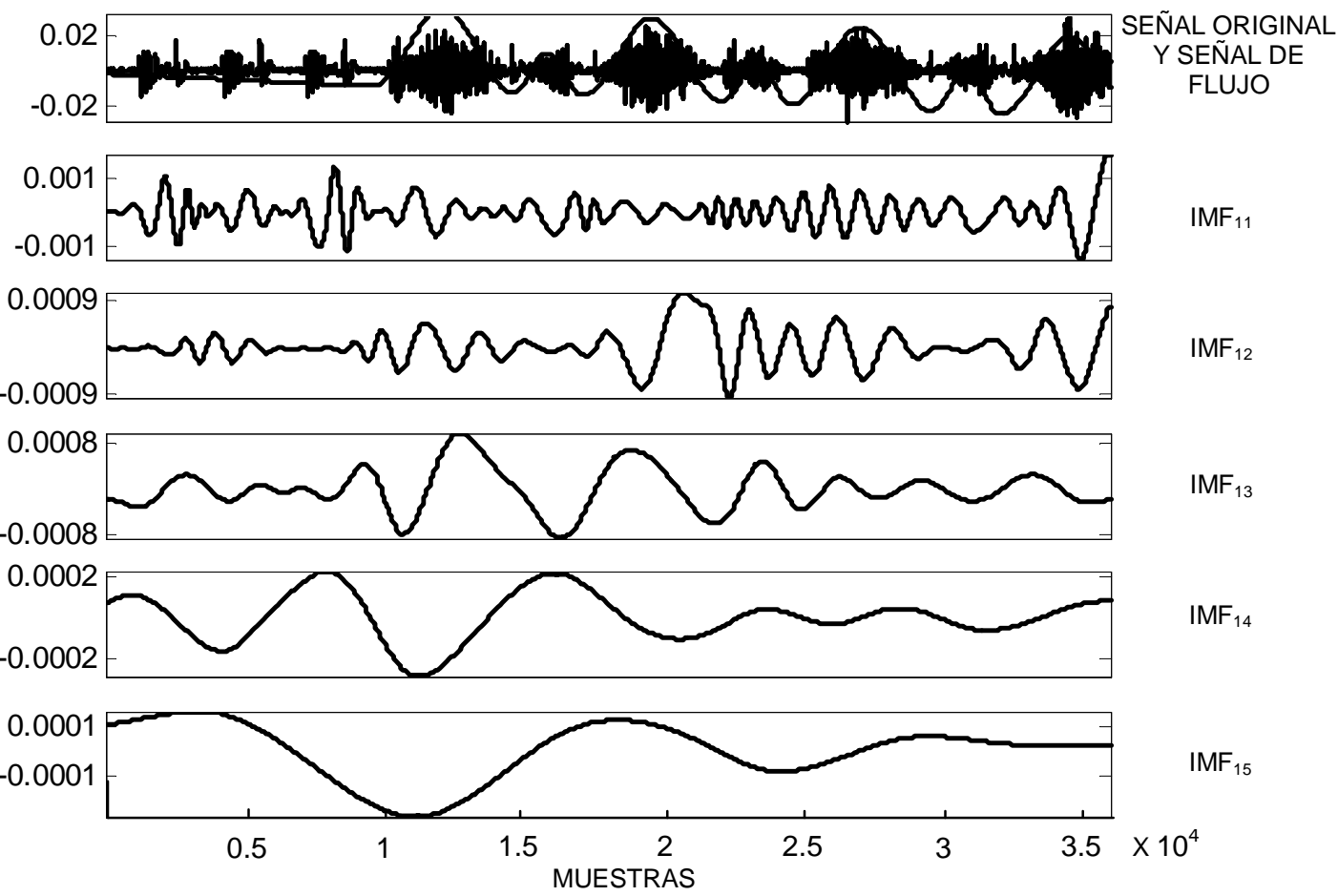

Figura 5.8 IMFs 11 al 15 de la señal acústica simulada con una relación señal-ruido de fondo de $50 \mathrm{~dB}$. 


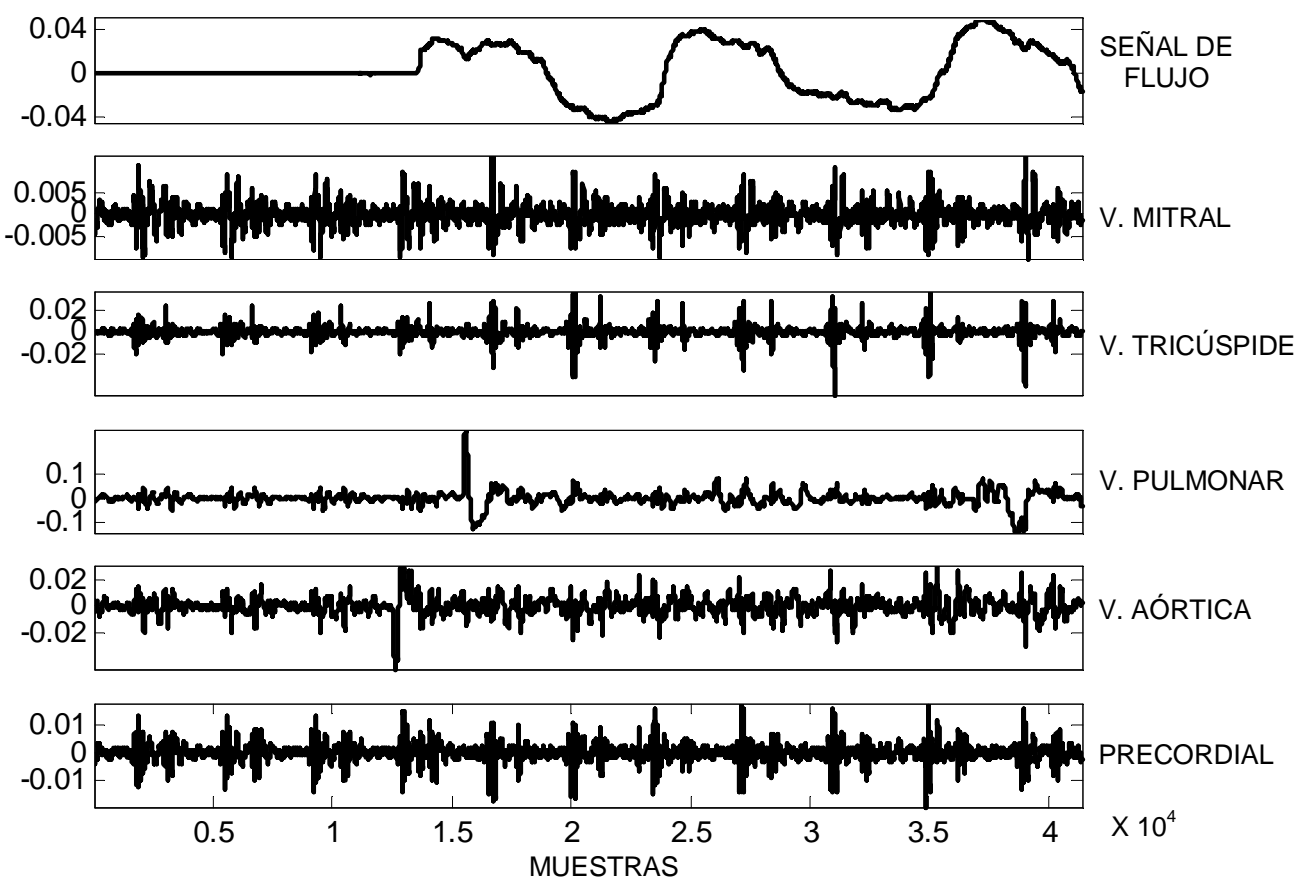

Figura 5.9 Señales acústicas obtenidas de las zonas de auscultación de las válvulas cardiacas a flujo de $0.5 \mathrm{~L} / \mathrm{seg}$.
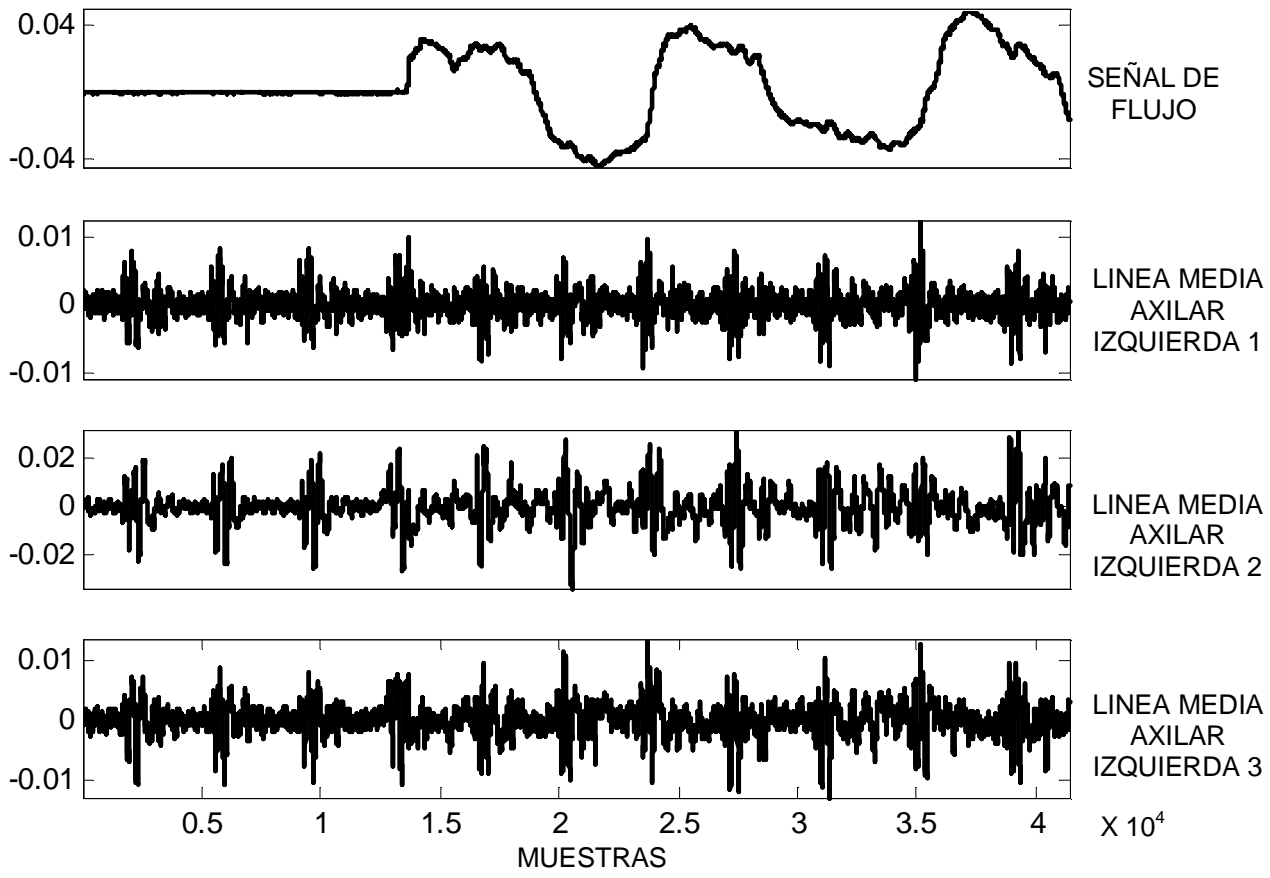

Figura 5.10 Señales acústicas de la línea media axilar izquierda a flujo de $0.5 \mathrm{~L} / \mathrm{seg}$. 

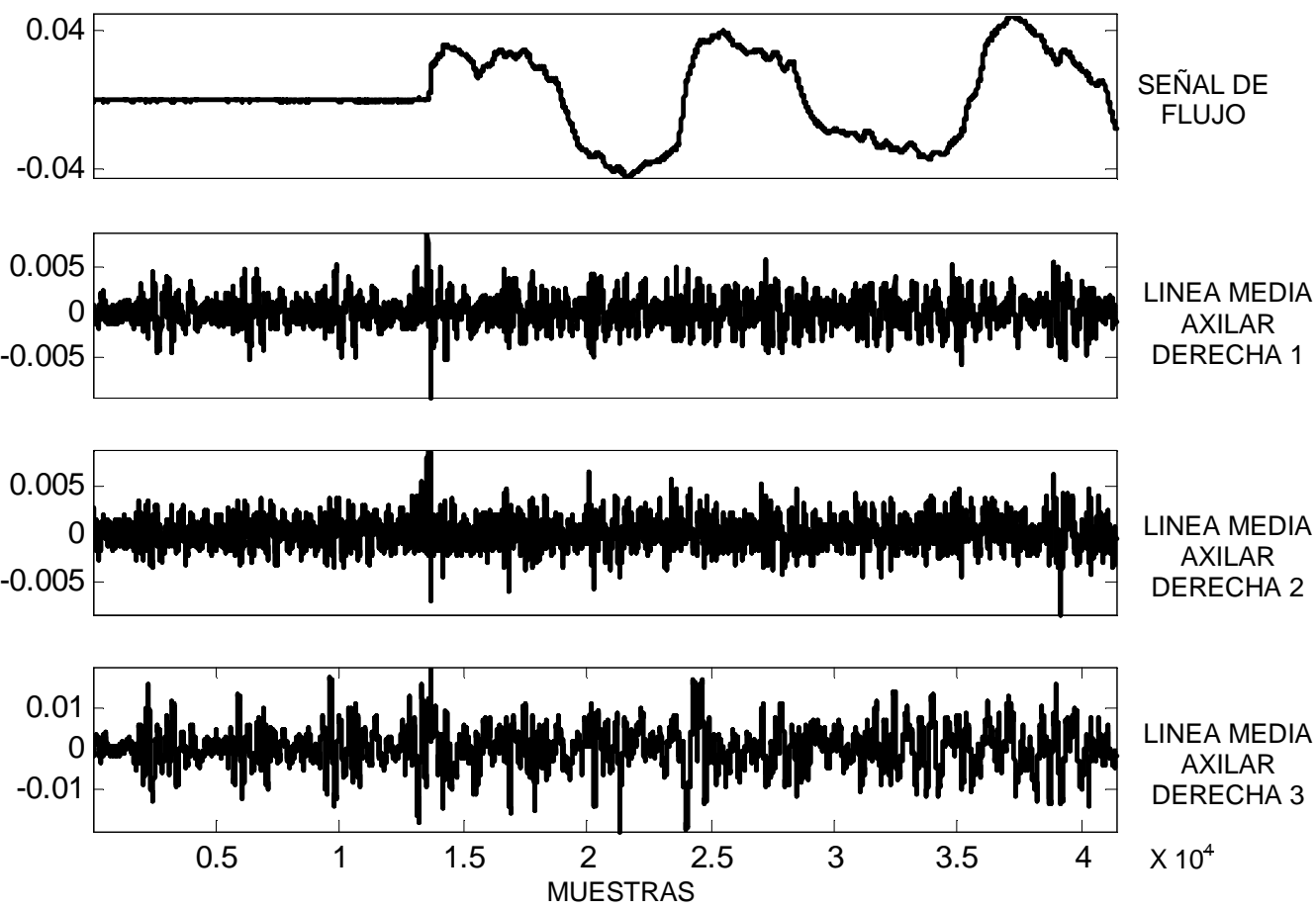

Figura 5.11 Señales acústicas de la línea media axilar derecha a flujo de $0.5 \mathrm{~L} / \mathrm{seg}$.
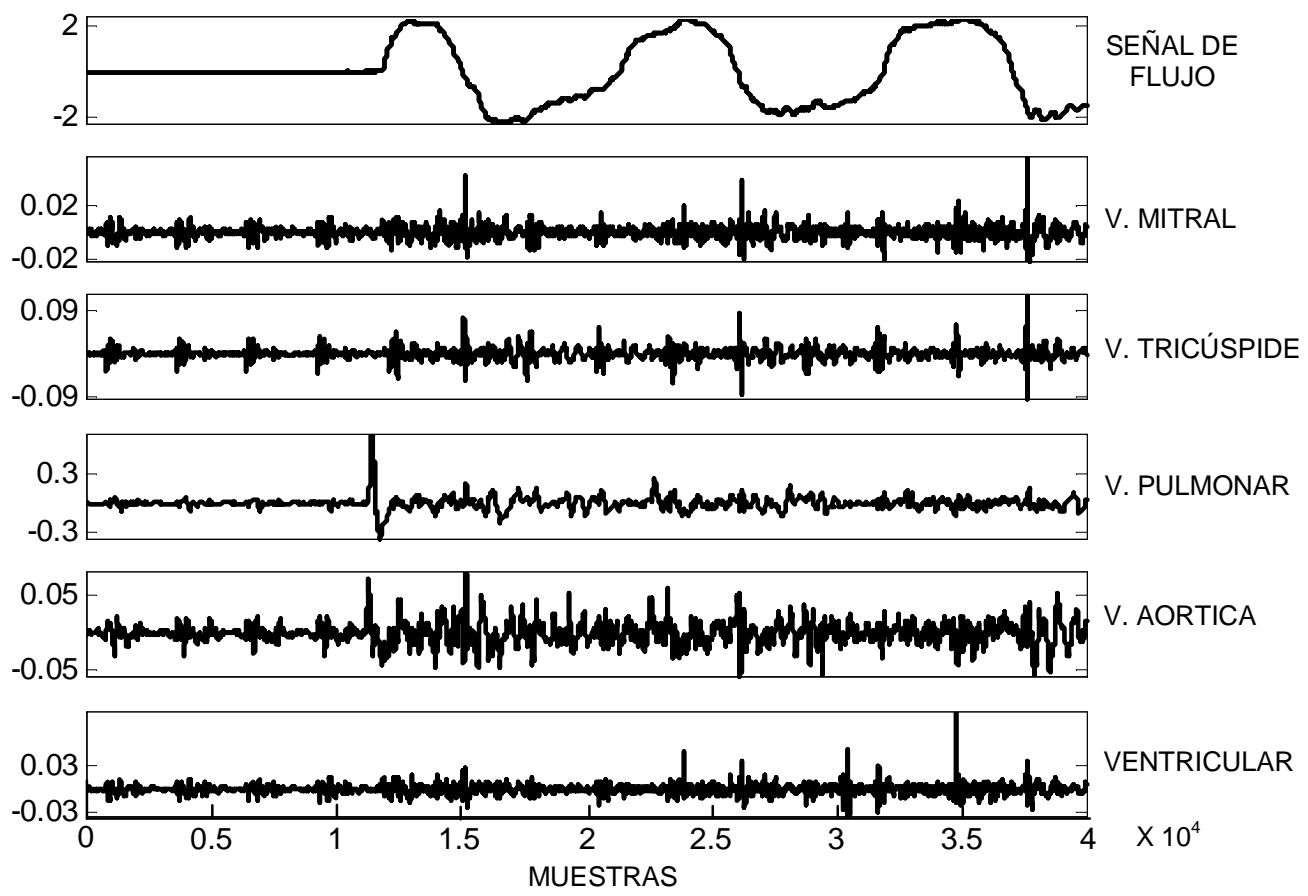

Figura 5.12 Señales acústicas obtenidas de las zonas de auscultación de las válvulas cardiacas a flujo de $2.0 \mathrm{~L} / \mathrm{seg}$. 

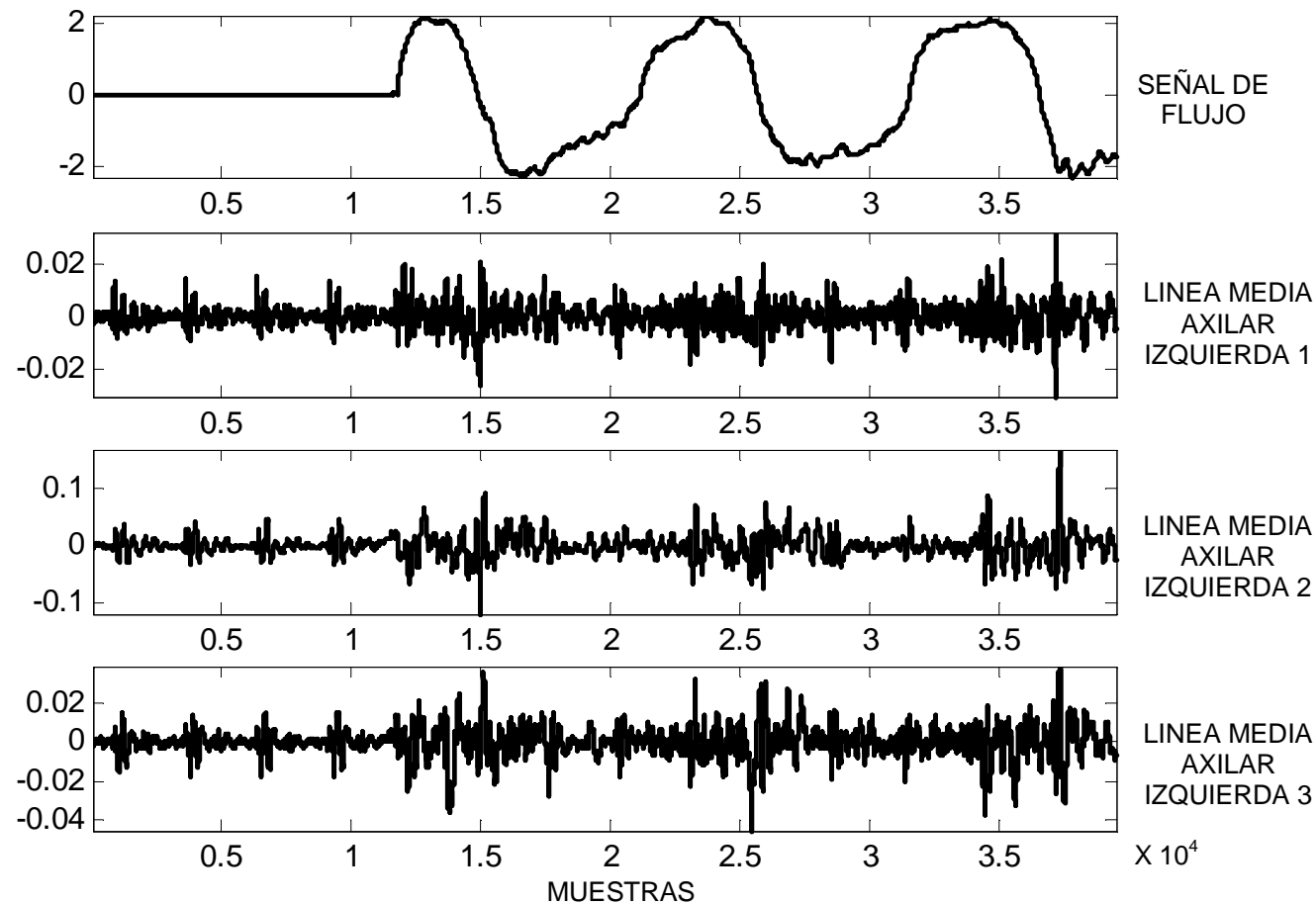

Figura 5.13 Señales acústicas de la línea media axilar izquierda a flujo $2.0 \mathrm{~L} / \mathrm{seg}$.

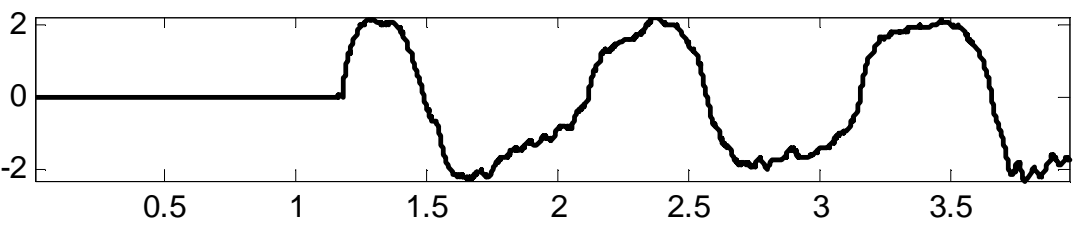

SEÑAL DE FLUJO

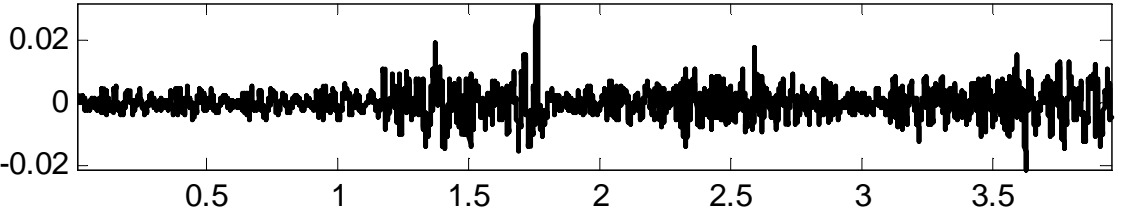

LINEA MEDIA AXILAR DERECHA 1

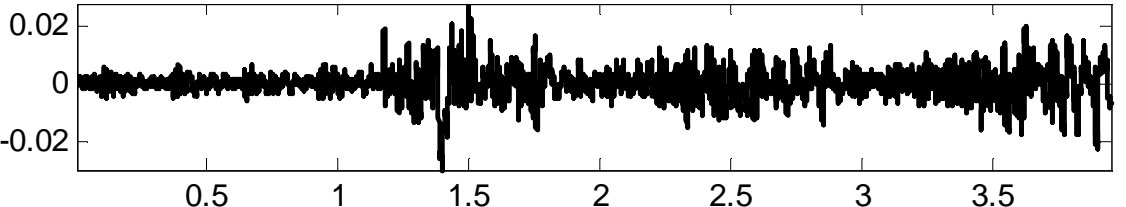
LINEA MEDIA AXILAR DERECHA 2

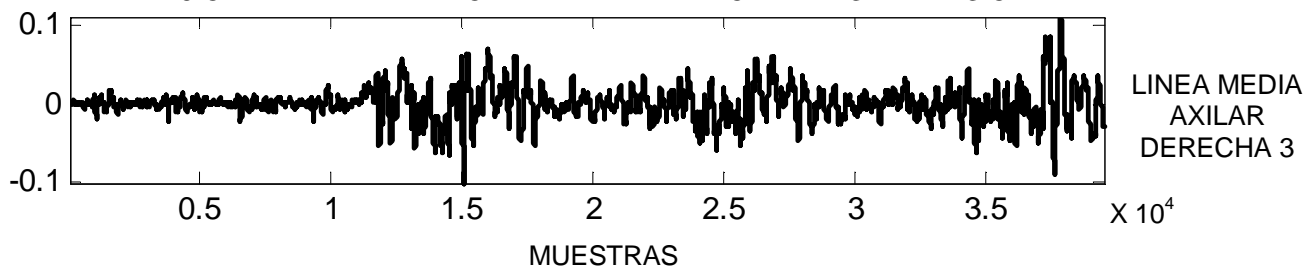

Figura 5.14 Señales acústicas de la línea media axilar derecha a flujo de $2.0 \mathrm{~L} / \mathrm{seg}$. 


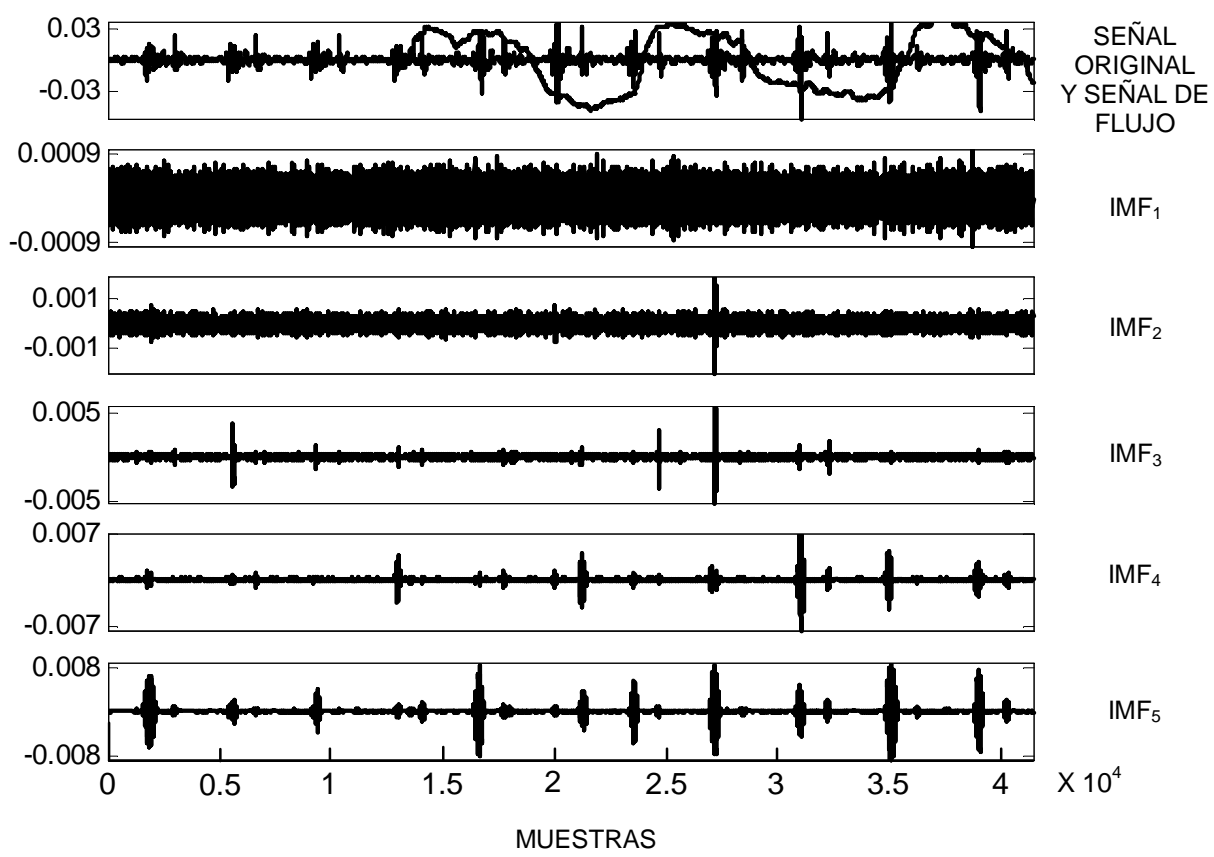

Figura 5.15 IMFs 1 al 5 de la señal acústica obtenida de un sujeto sano en la zona de auscultación que corresponde a la válvula tricúspide a flujo de $0.5 \mathrm{~L} / \mathrm{seg}$.

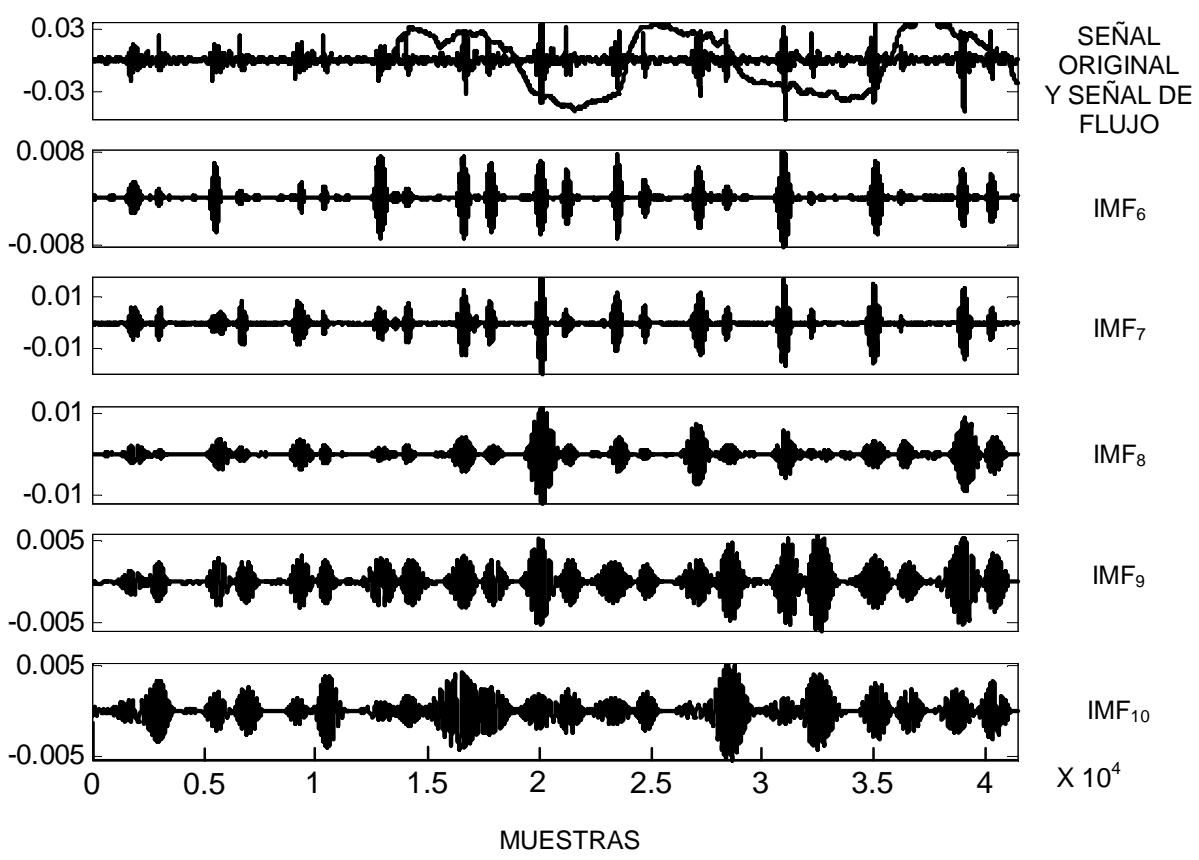

Figura 5.16 IMFs 6 al 10 de la señal acústica obtenida de un sujeto sano en la zona de auscultación que corresponde a la válvula tricúspide a flujo de $0.5 \mathrm{~L} / \mathrm{seg}$. 


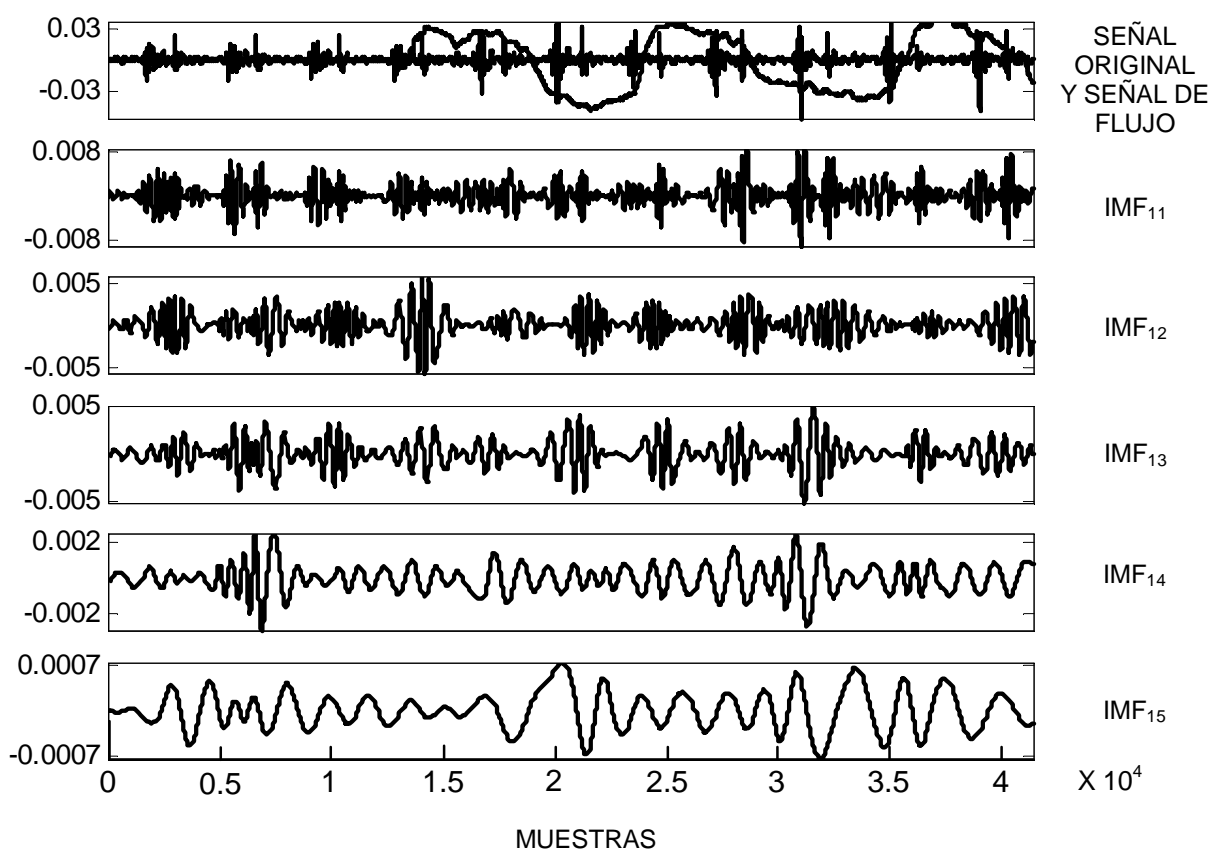

Figura 5.17 IMFs 11 al 15 de la señal acústica obtenida de un sujeto sano en la zona de auscultación que corresponde a la válvula tricúspide a flujo de $0.5 \mathrm{~L} / \mathrm{seg}$.

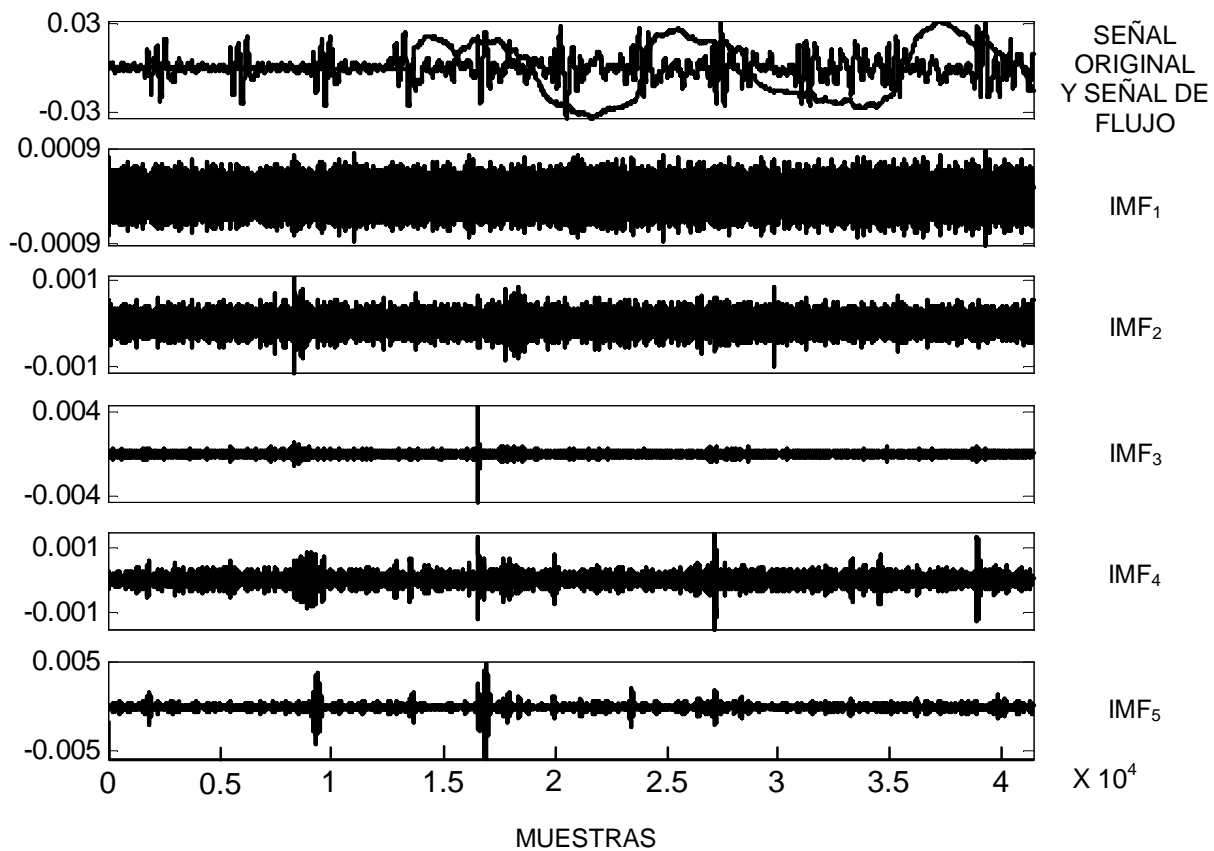

Figura 5.18 IMFs 1 al 5 de la señal acústica obtenida de un sujeto sano en la línea axilar izquierda media a flujo de $0.5 \mathrm{~L} / \mathrm{seg}$. 


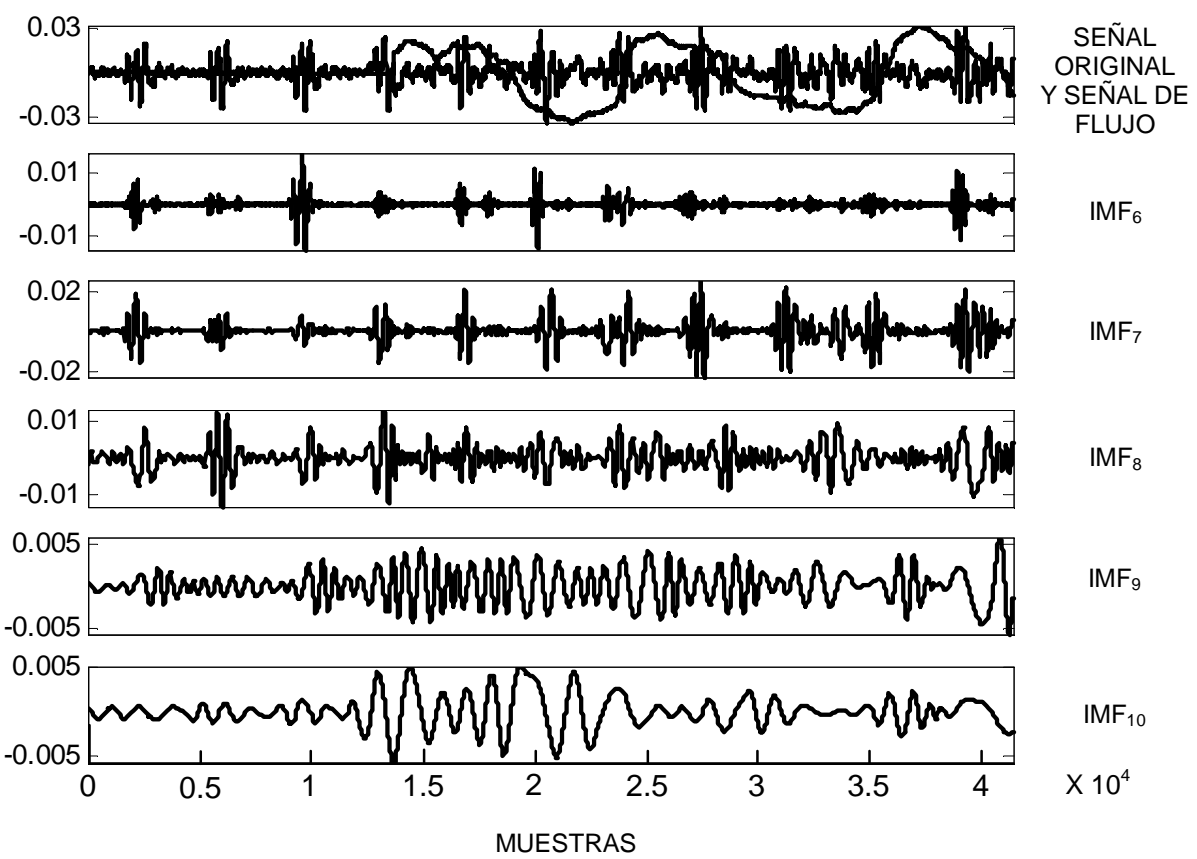

Figura 5.19 IMFs 6 al 10 de la señal acústica obtenida de un sujeto sano en la línea axilar izquierda media a flujo de $0.5 \mathrm{~L} / \mathrm{seg}$.

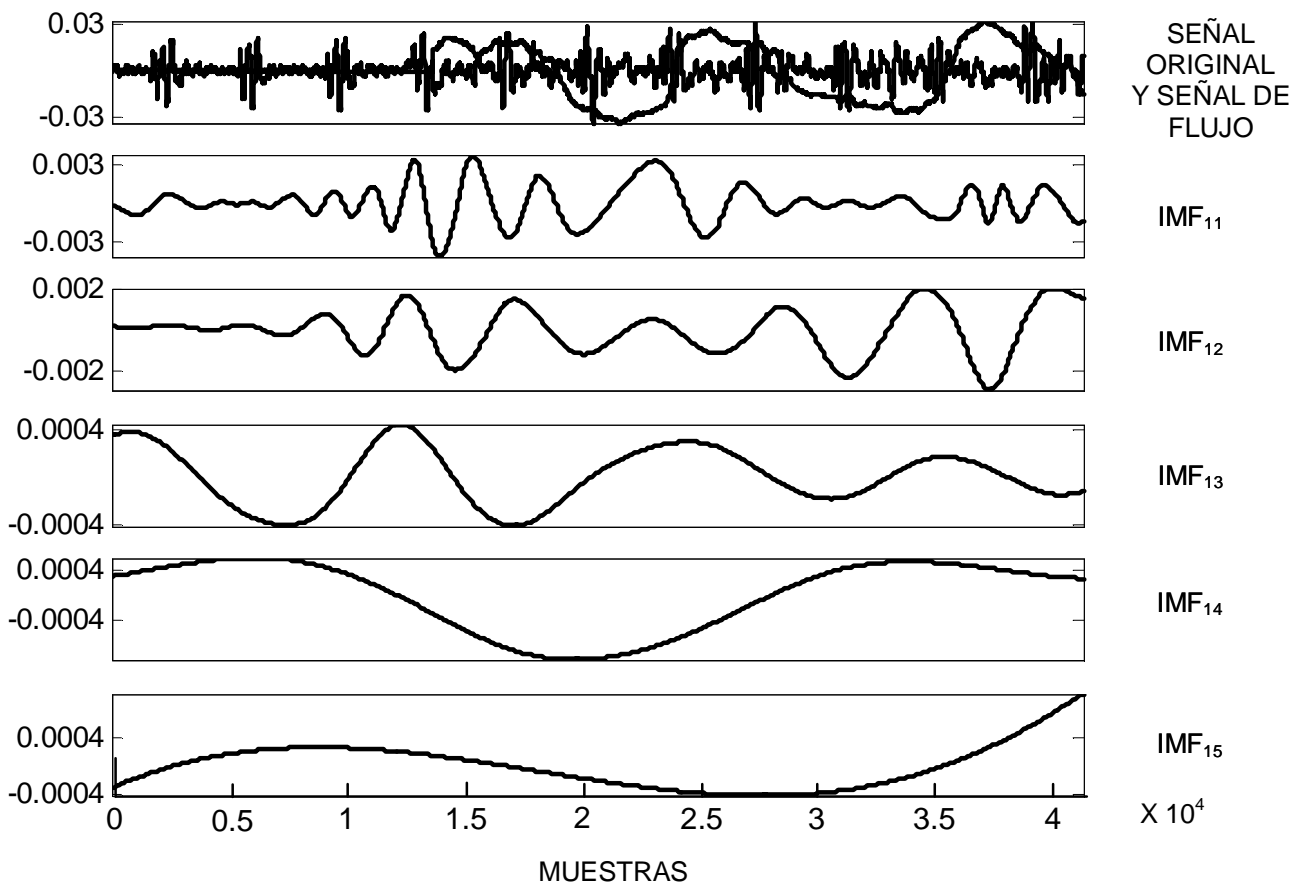

Figura 5.20 IMFs 11 al 15 de la señal acústica obtenida de un sujeto sano en la línea axilar izquierda media a flujo de $0.5 \mathrm{~L} / \mathrm{seg}$. 


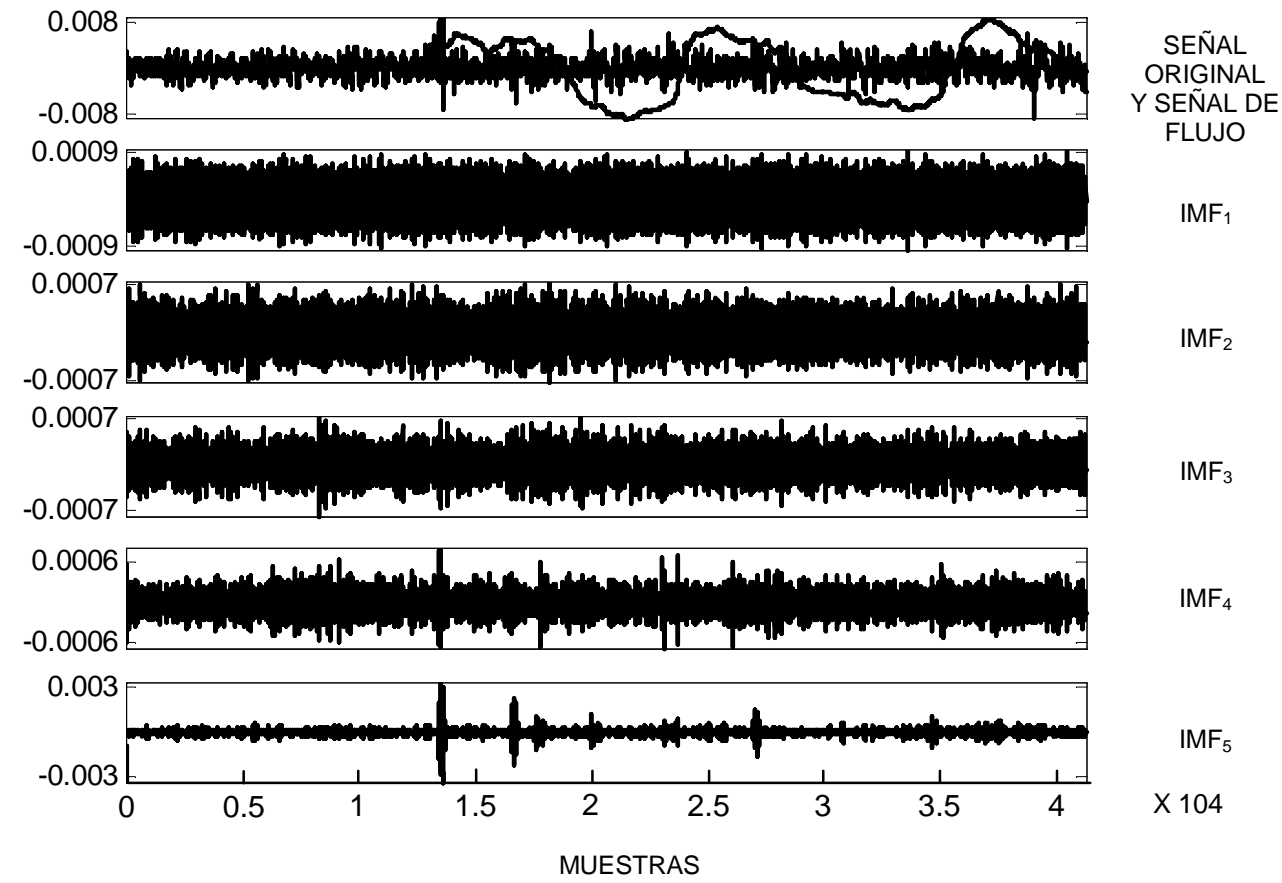

Figura 5.21 IMFs 1 al 5 de la señal acústica obtenida de un sujeto sano en la línea axilar derecha media a flujo de $0.5 \mathrm{~L} / \mathrm{seg}$.

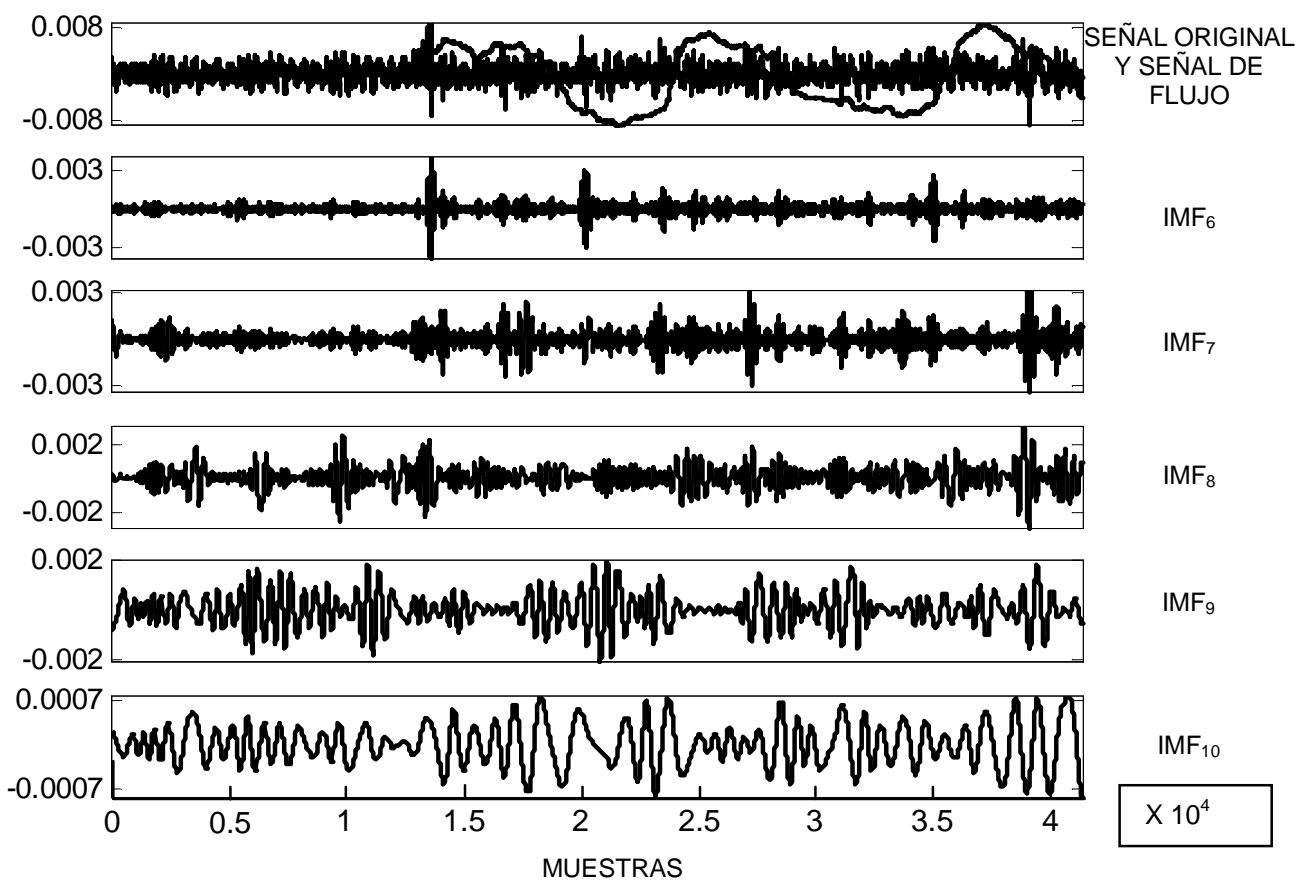

Figura 5.22 IMFs 6 al 10 de la señal acústica obtenida de un sujeto sano en la línea axilar derecha media a flujo de $0.5 \mathrm{~L} / \mathrm{seg}$. 


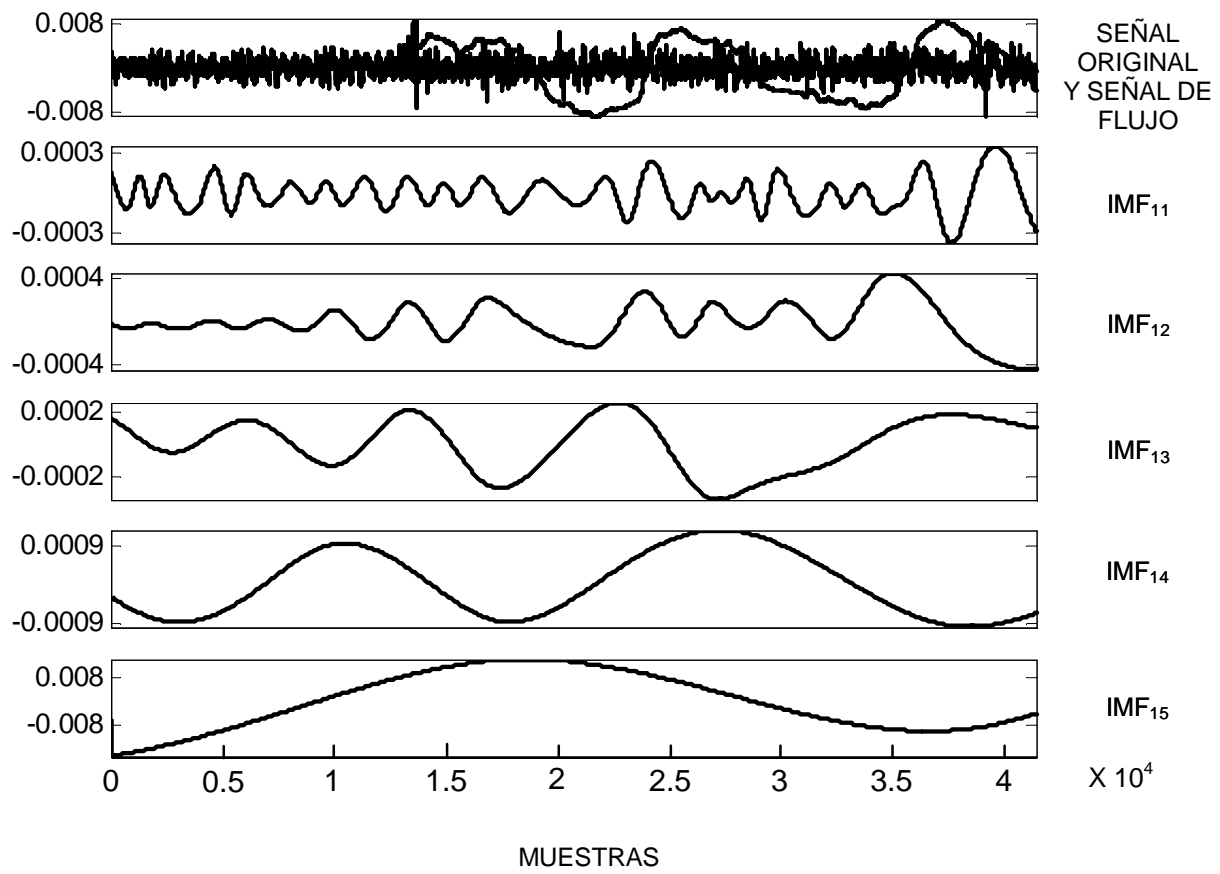

Figura 5.23 IMFs 11 al 15 de la señal acústica obtenida de un sujeto sano en la línea axilar derecha media a flujo de $0.5 \mathrm{~L} / \mathrm{seg}$.

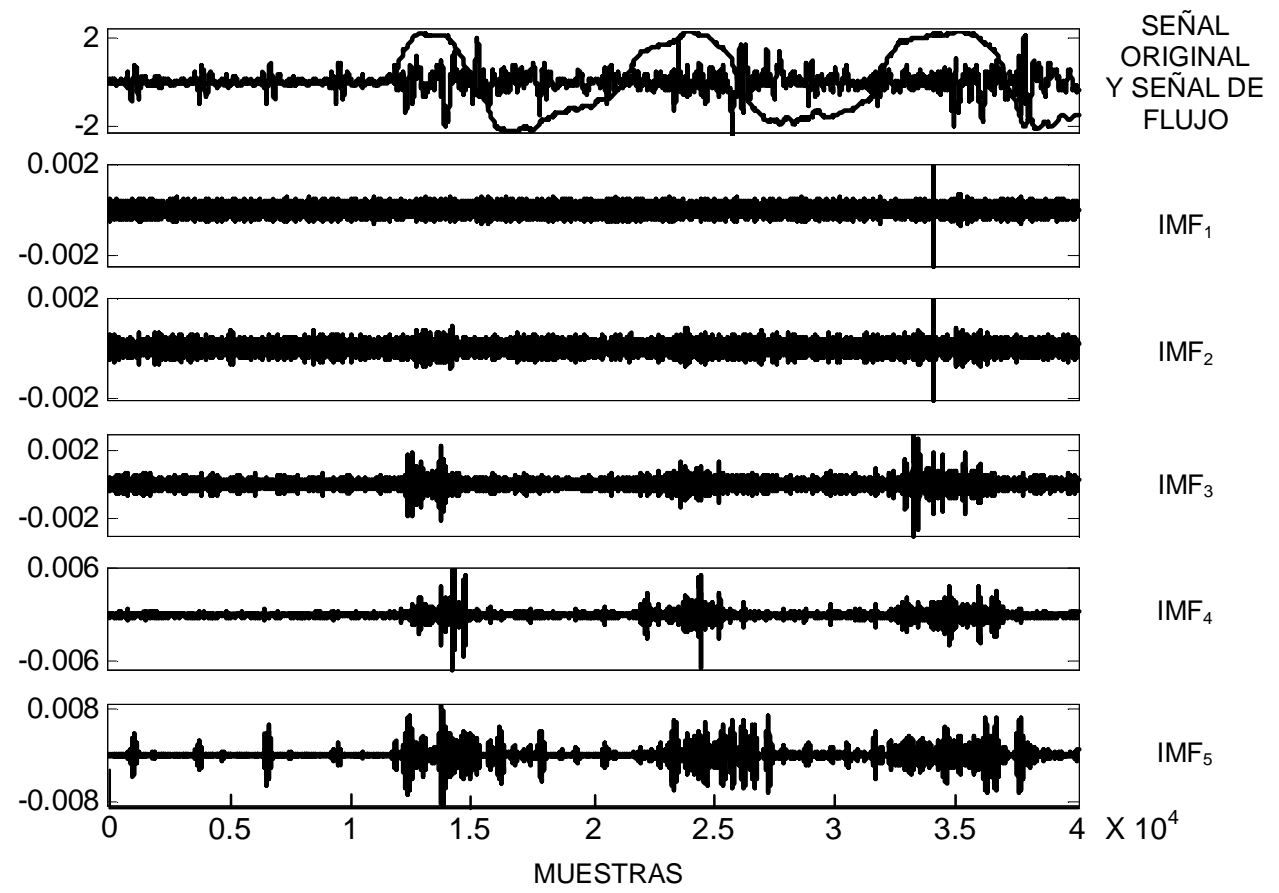

Figura 5.24 IMFs 1 al 5 de la señal acústica obtenida de un sujeto sano en la línea axilar izquierda basal a flujo de $2 \mathrm{~L} / \mathrm{seg}$. 


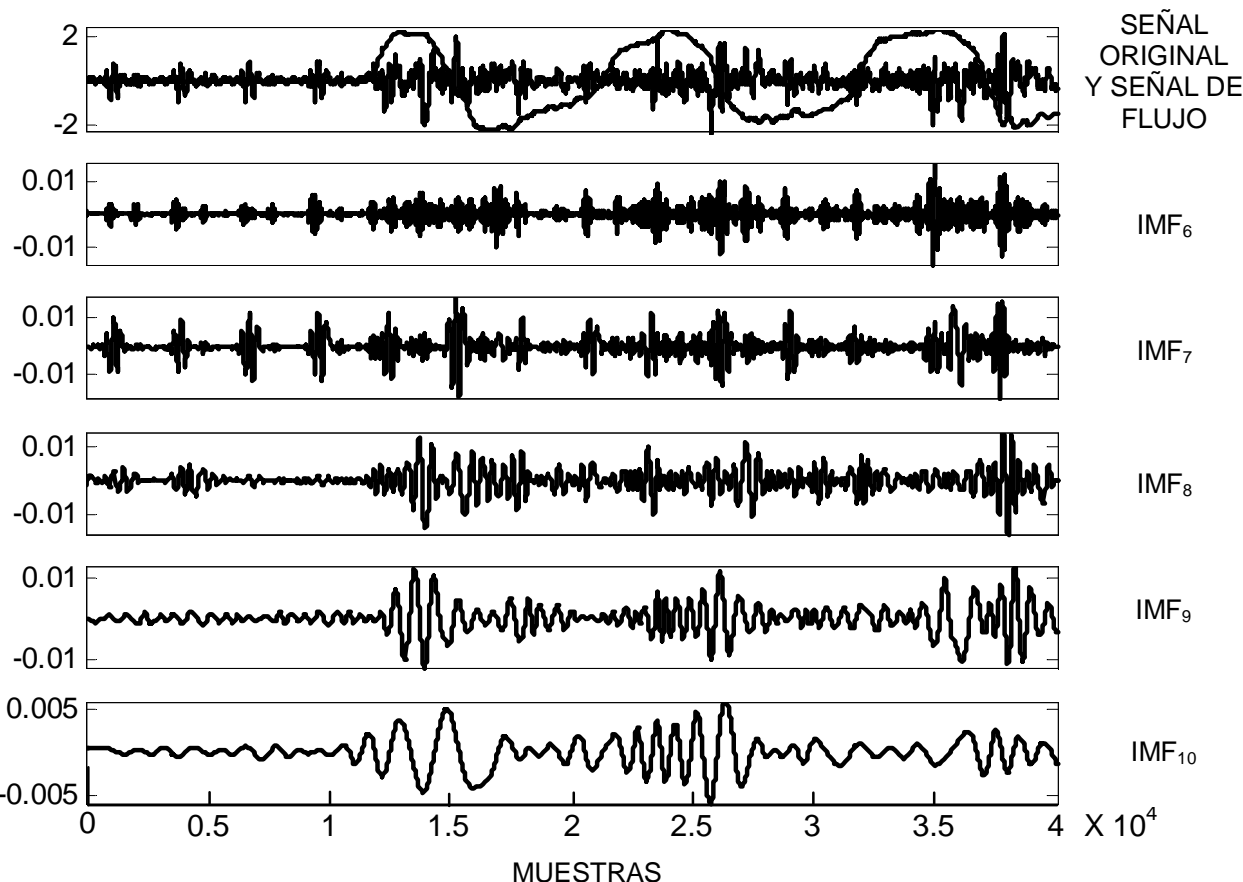

Figura 5.25 IMFs 6 al 10 de la señal acústica obtenida de un sujeto sano en la línea axilar izquierda basal a flujo de $2 \mathrm{~L} / \mathrm{seg}$.

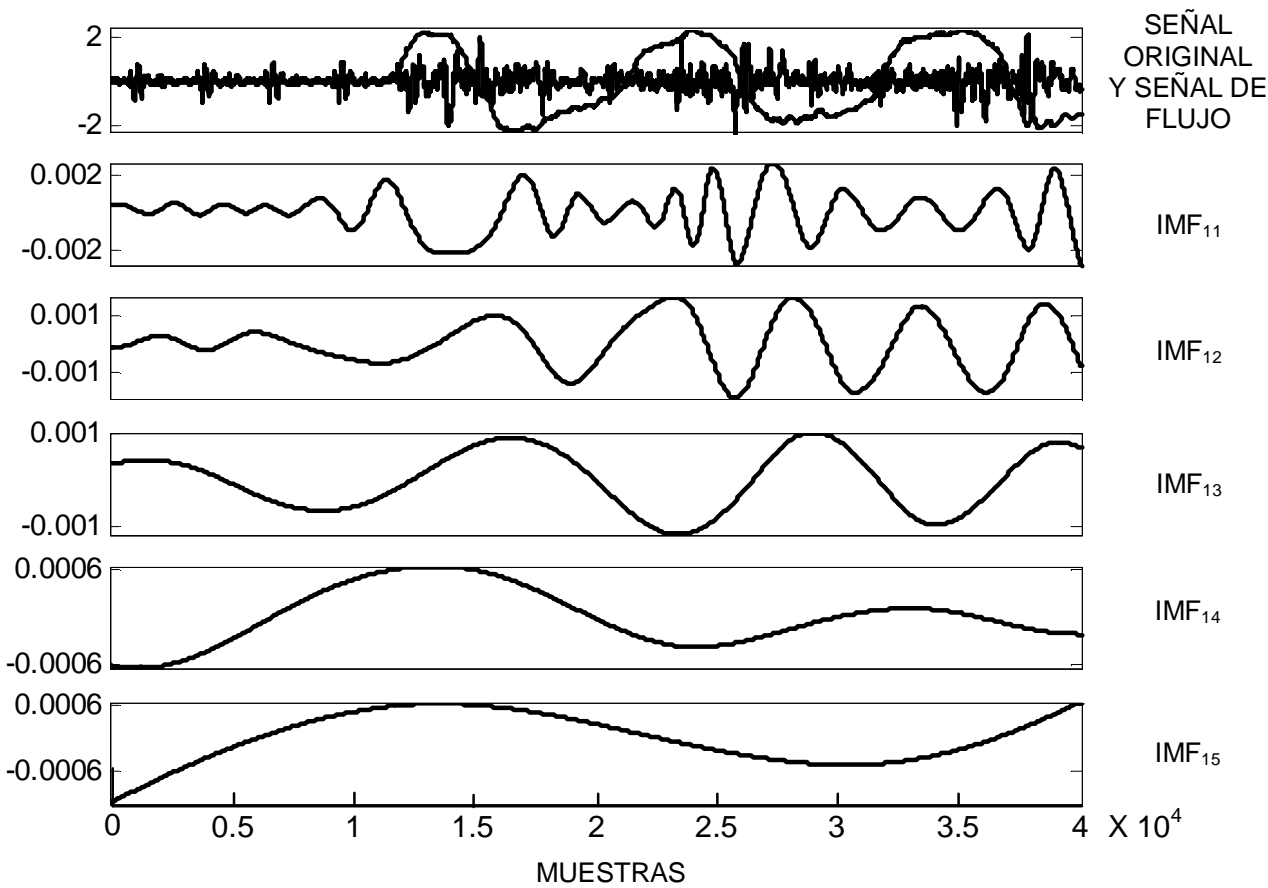

Figura 5.26 IMF's 11 al 15 de la señal acústica obtenida de un sujeto sano en la línea axilar izquierda basal a flujo de $2 \mathrm{~L} / \mathrm{seg}$. 

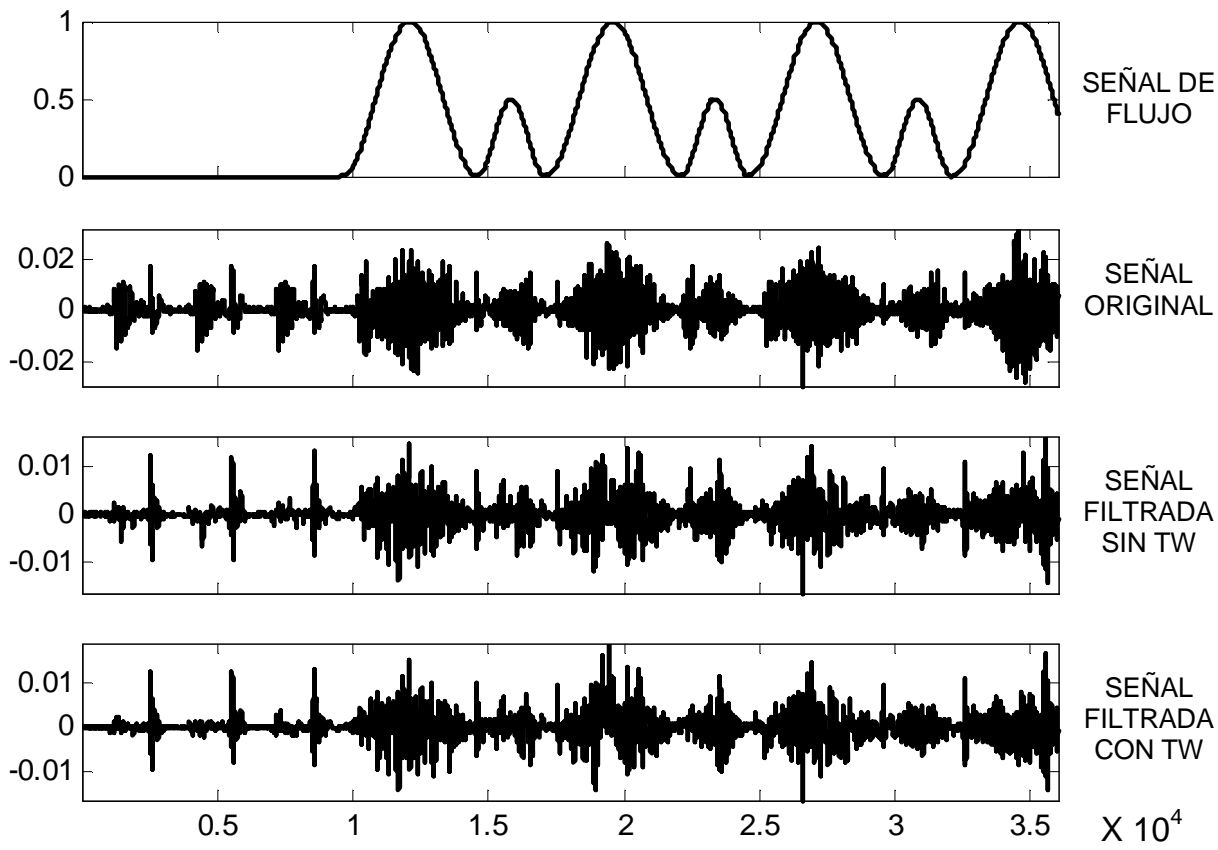

Figura 5.27 Señal simulada con una relación señal respiratoria-ruido cardiaco de $50 \mathrm{~dB}$ y sus señales filtradas.
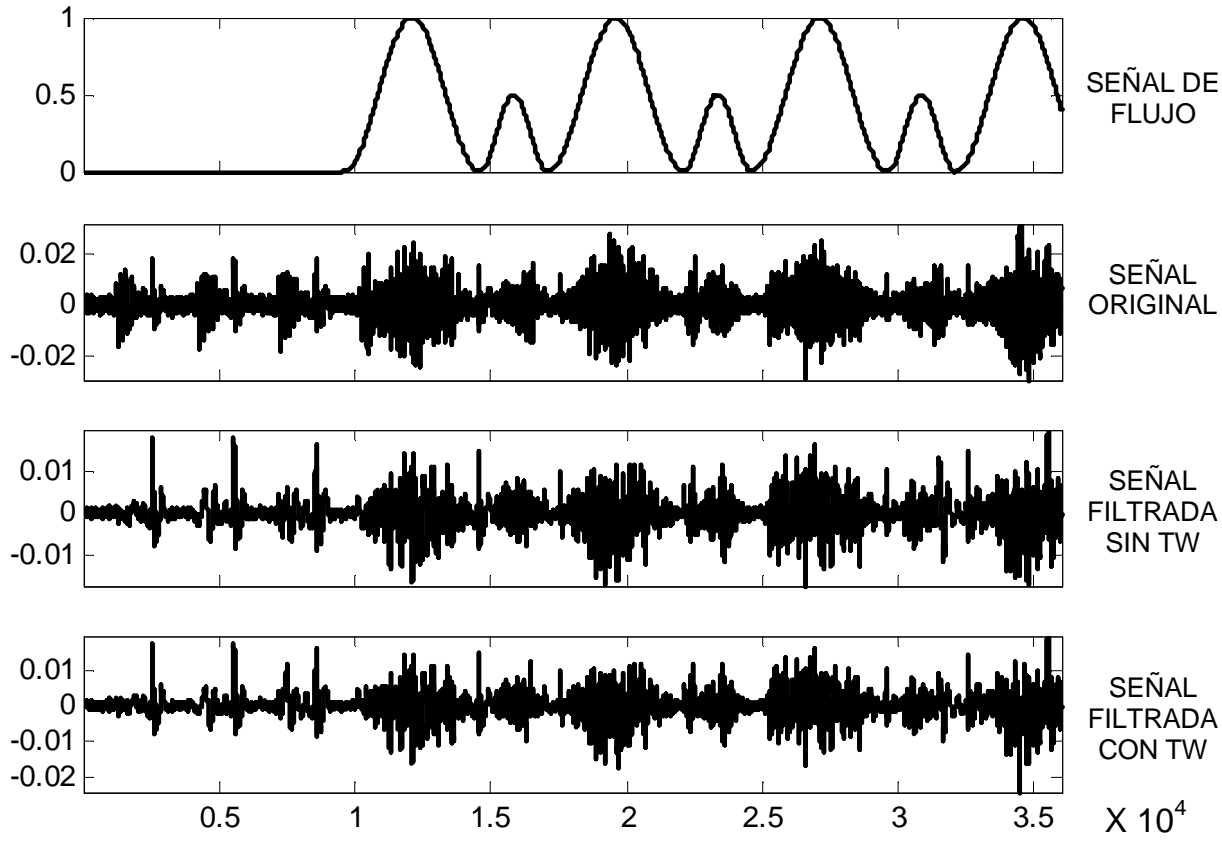

Figura 5.28 Señal simulada con una relación señal respiratoria-ruido cardiaco de $20 \mathrm{~dB}$ y sus señales filtradas. 

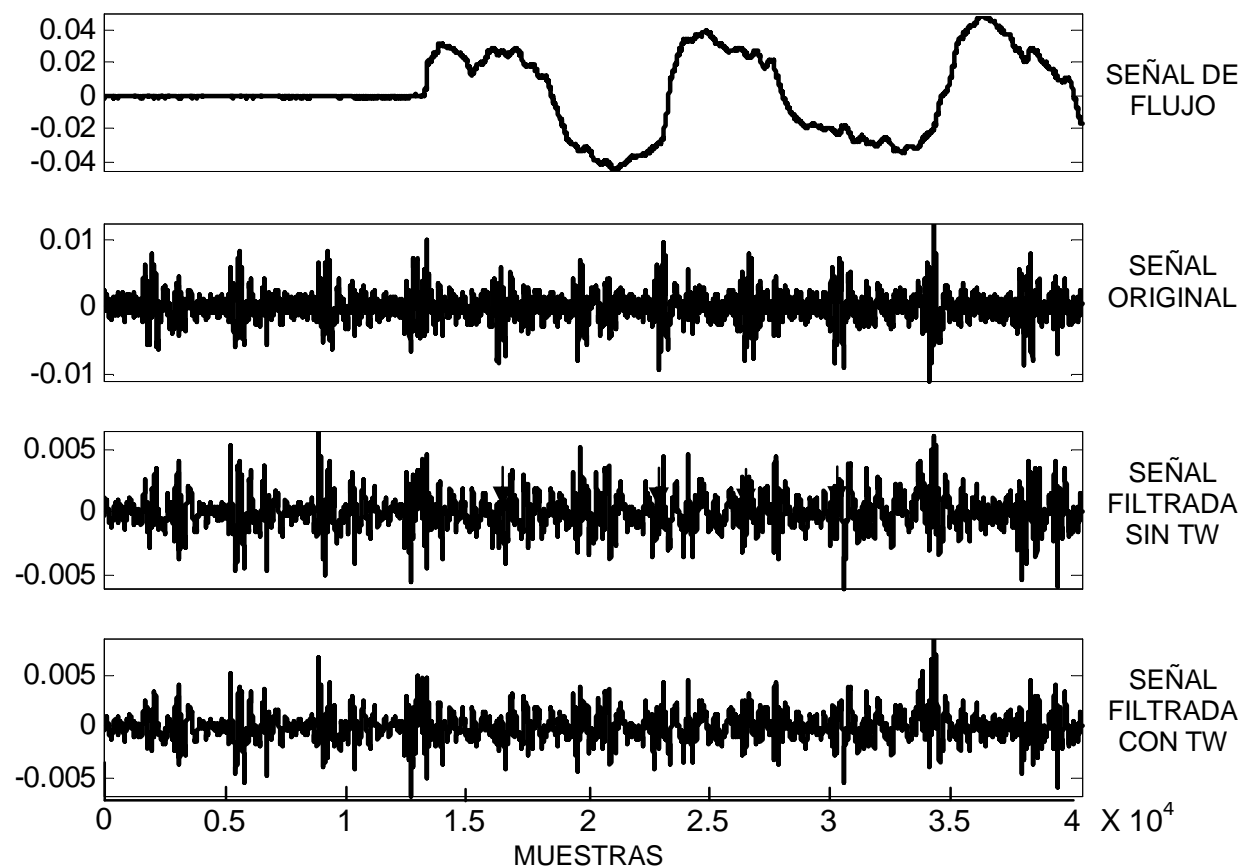

Figura 5.29 Señal obtenida de la línea axilar izquierda apical y sus señales filtradas a flujo de 0.5 L/seg.
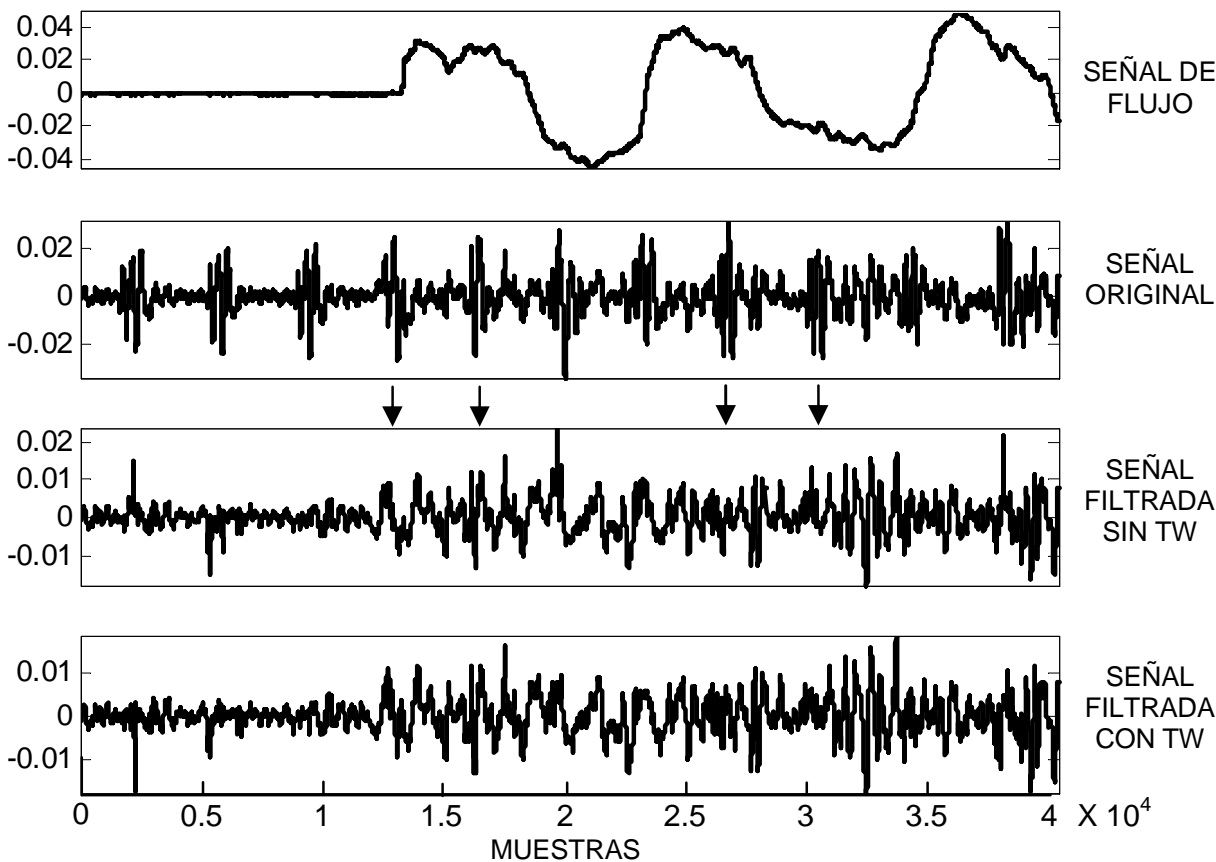

Figura 5.30 Señal obtenida de la línea axilar izquierda media y sus señales filtradas a flujo de $0.5 \mathrm{~L} / \mathrm{seg}$. 


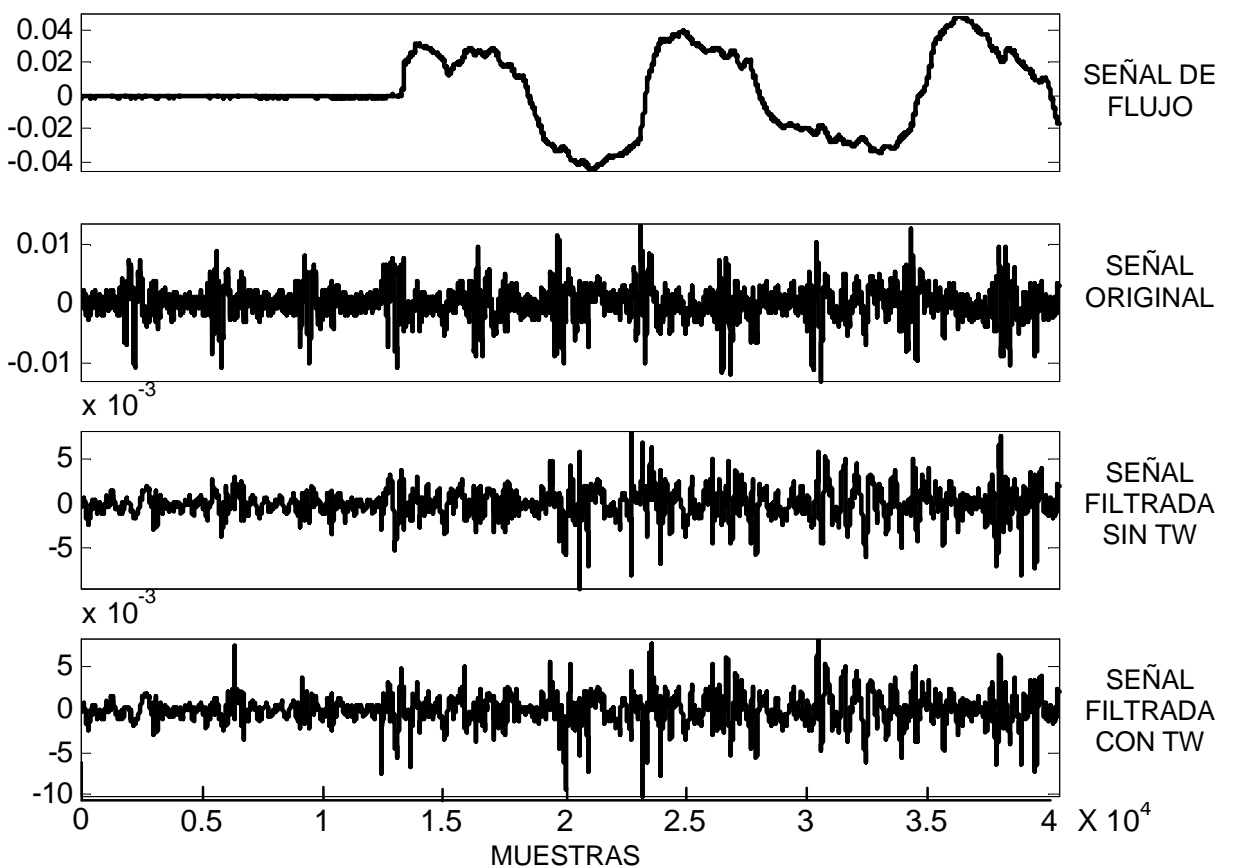

Figura 5.31 Señal obtenida de la línea axilar izquierda basal y sus señales filtradas a flujo de $0.5 \mathrm{~L} / \mathrm{seg}$.
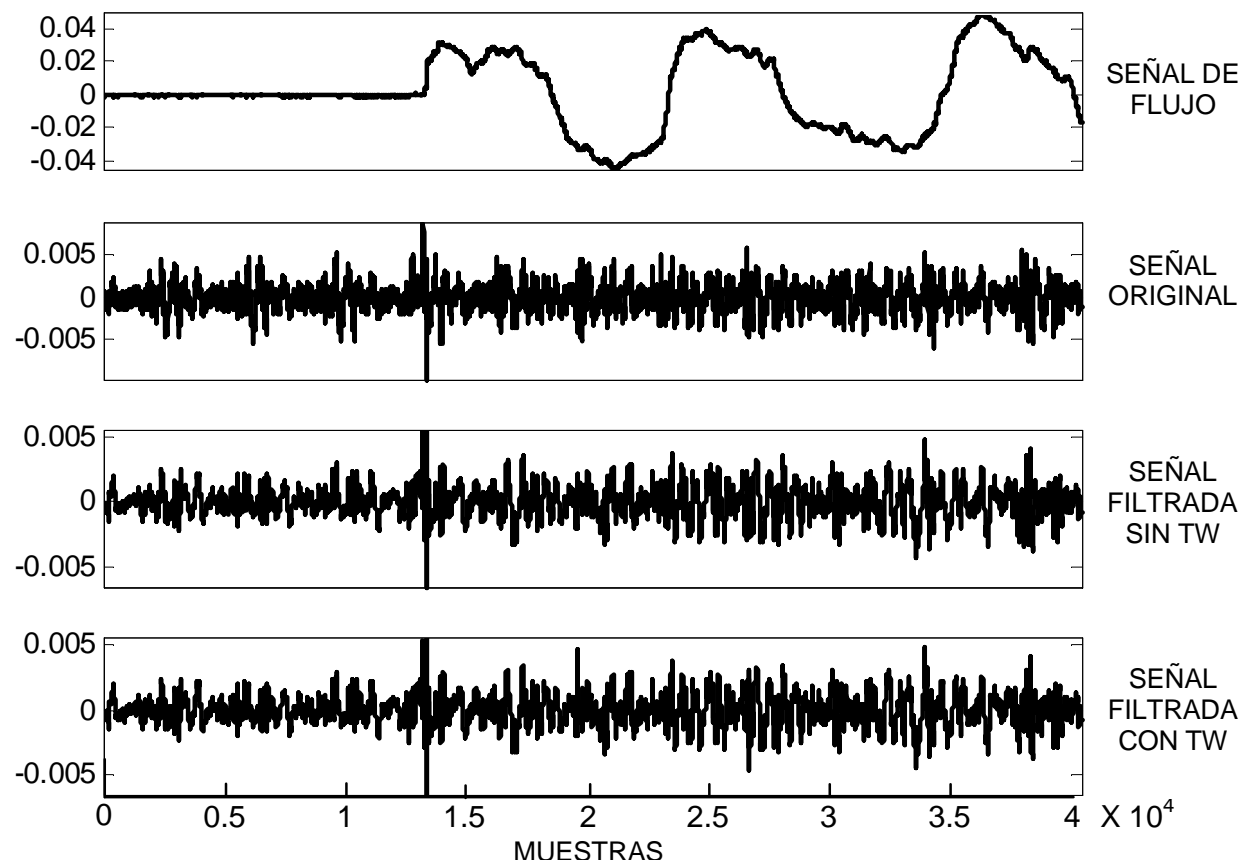

Figura 5.32 Señal obtenida de la línea axilar derecha apical y sus señales filtradas a flujo de $0.5 \mathrm{~L} / \mathrm{seg}$. 

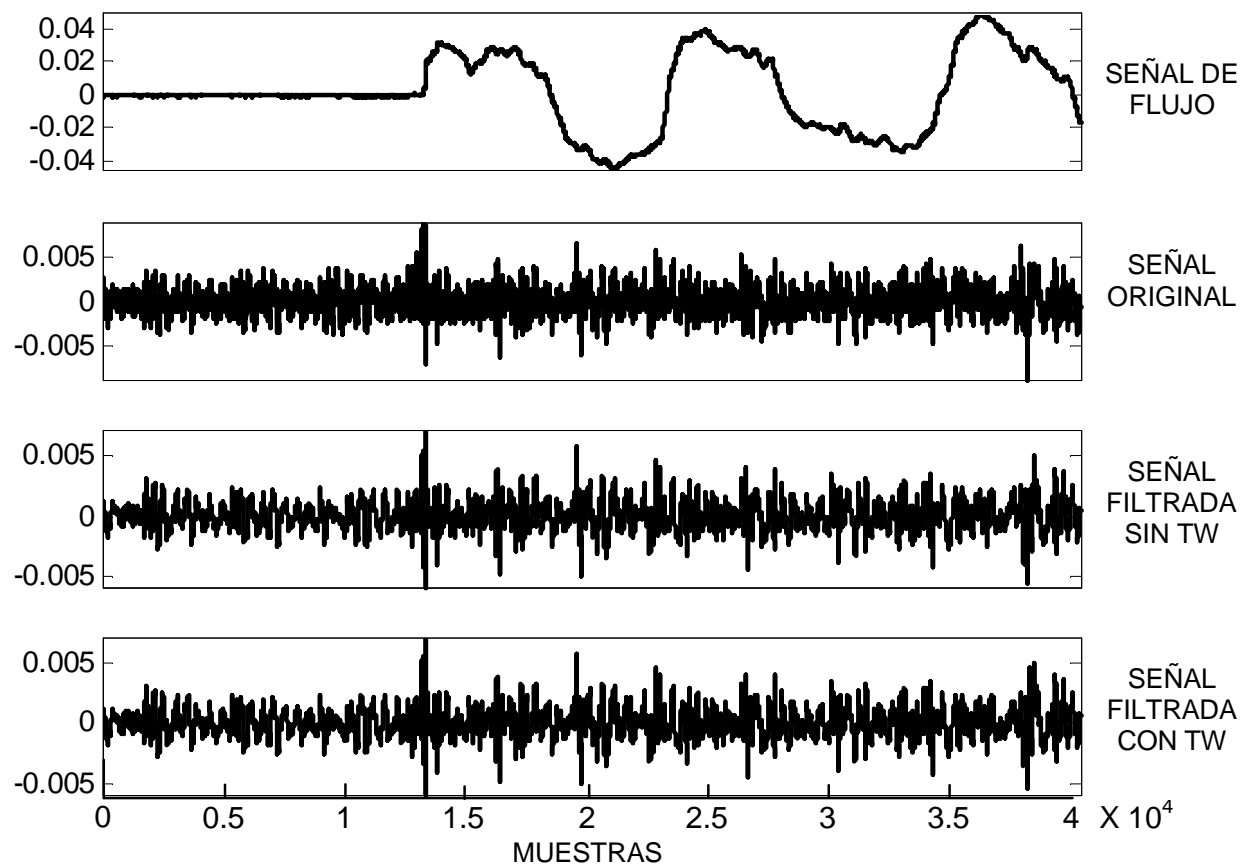

Figura 5.33 Señal obtenida de la línea axilar derecha media y sus señales filtradas a flujo de $0.5 \mathrm{~L} / \mathrm{seg}$.
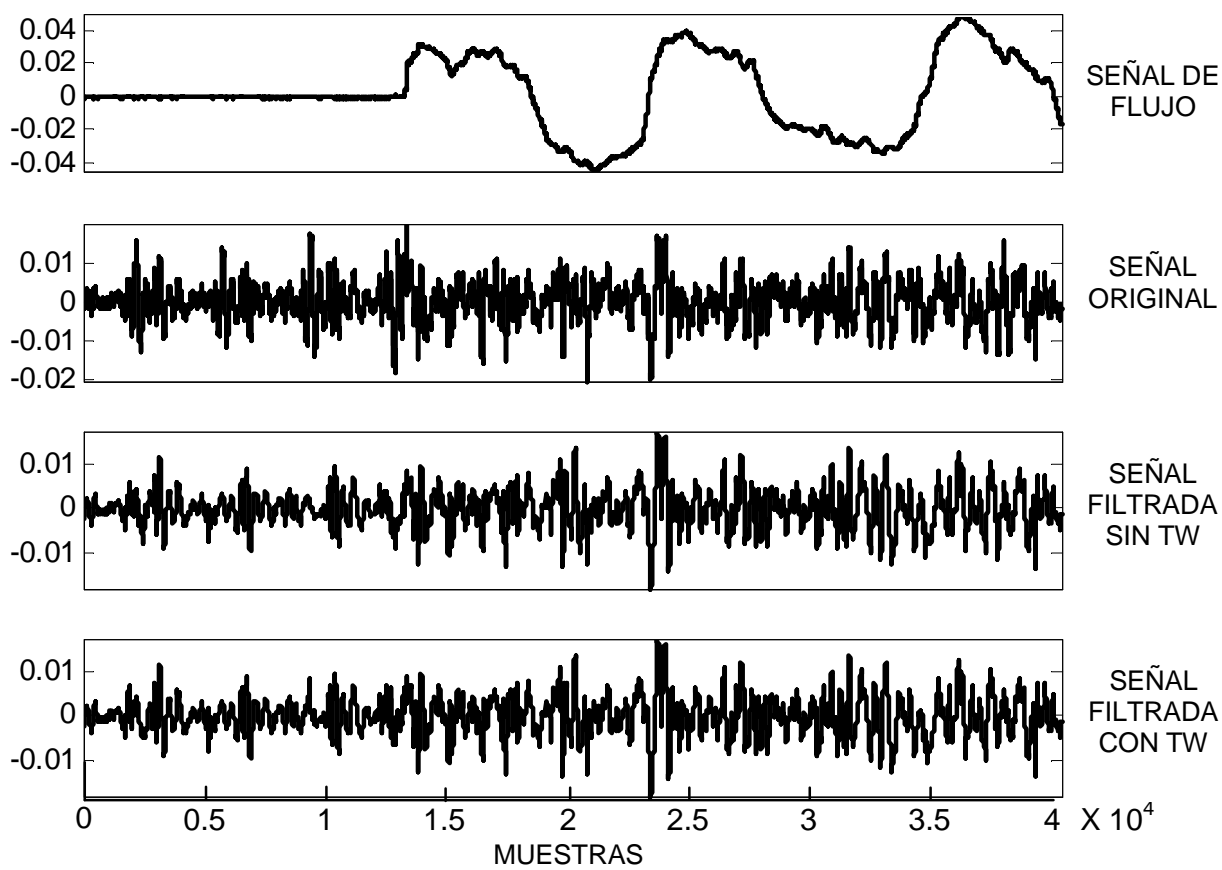

Figura 5.34 Señal obtenida de la línea axilar derecha basal y sus señales filtradas a flujo de $0.5 \mathrm{~L} / \mathrm{seg}$. 

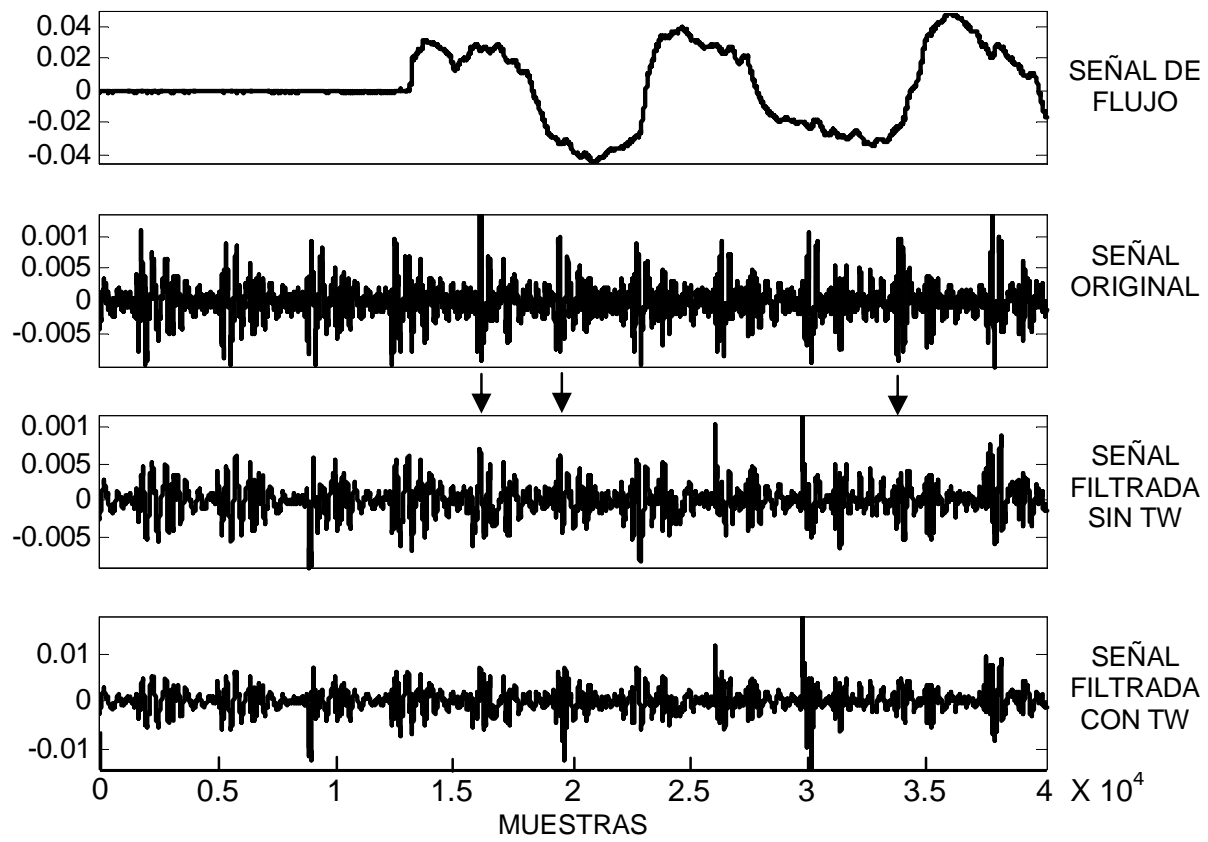

Figura 5.35 Señal obtenida de la zona de auscultación de la válvula mitral y sus señales filtradas a flujo de $0.5 \mathrm{~L} / \mathrm{seg}$.

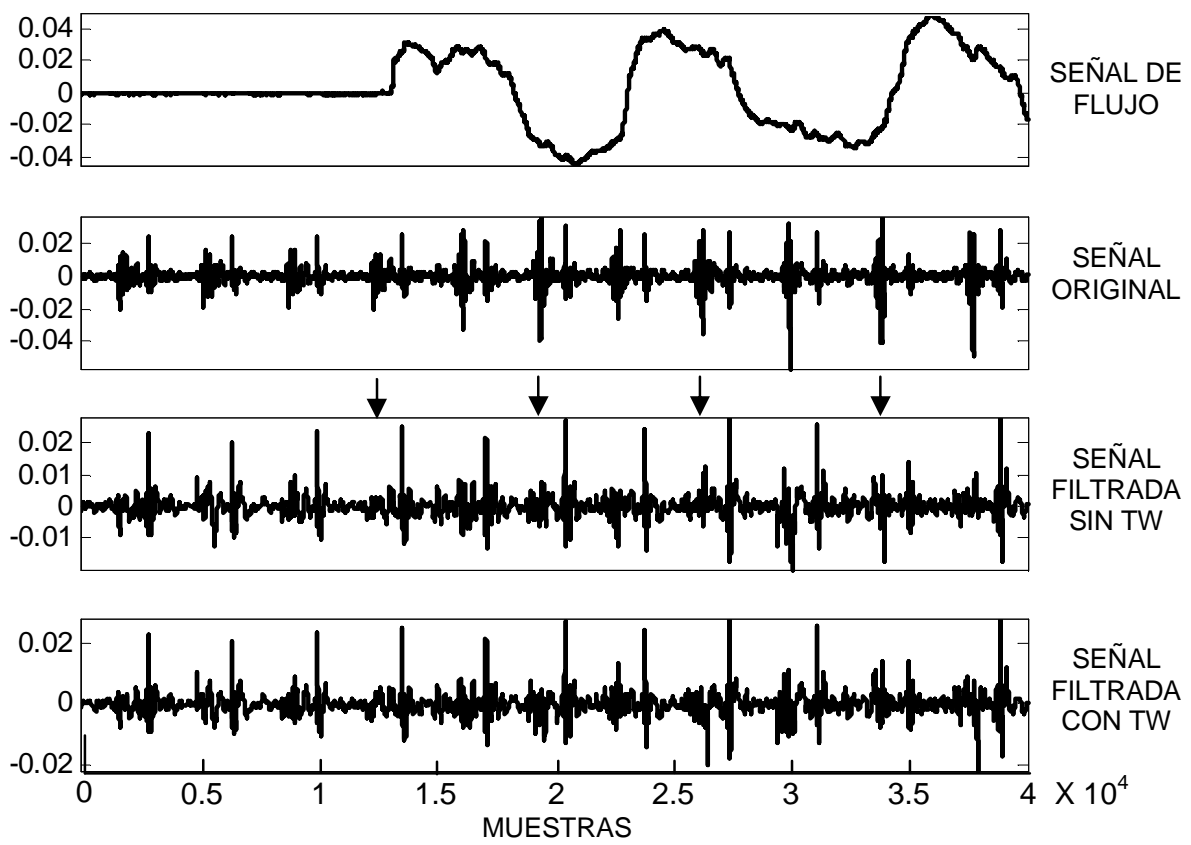

Figura 5.36 Señal obtenida de la zona de auscultación de la válvula tricúspide y sus señales filtradas a flujo de $0.5 \mathrm{~L} / \mathrm{seg}$. 

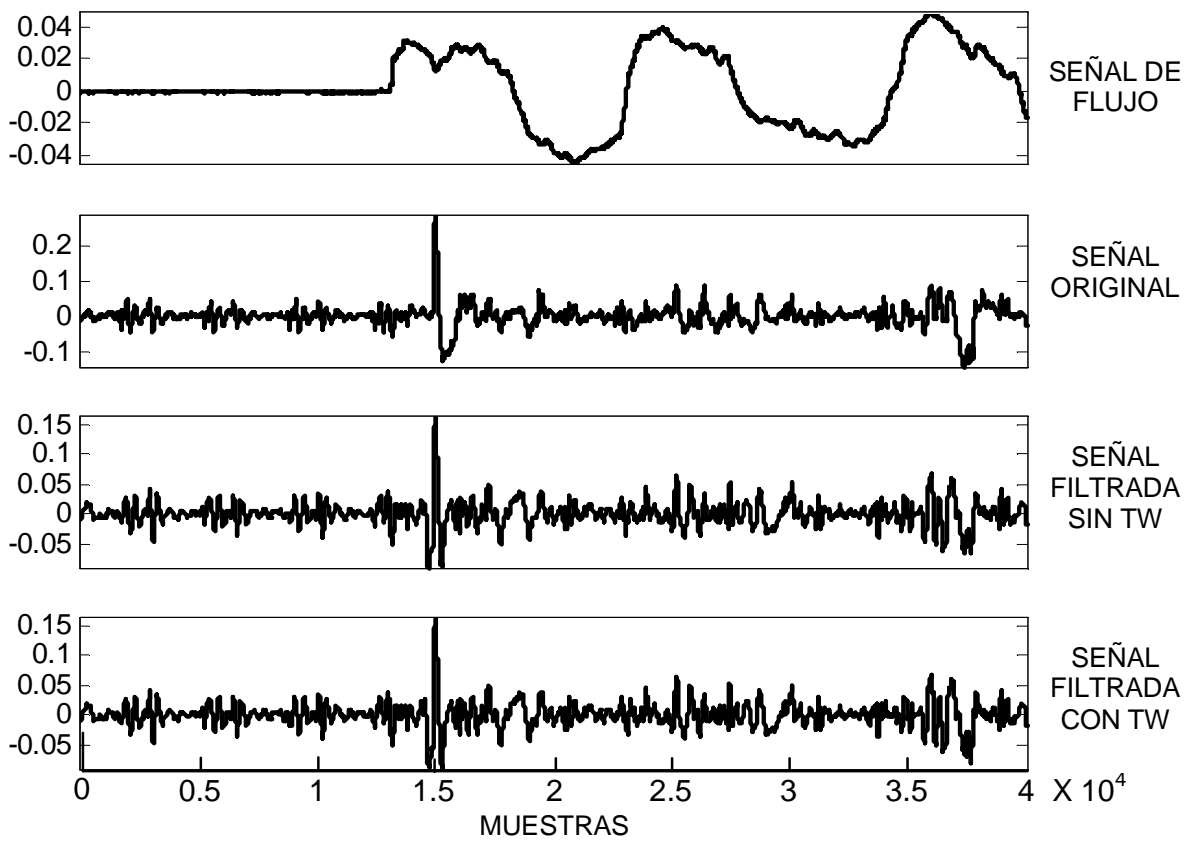

Figura 5.37 Señal obtenida de la zona de auscultación de la válvula pulmonar y sus señales filtradas a flujo de $0.5 \mathrm{~L} / \mathrm{seg}$.
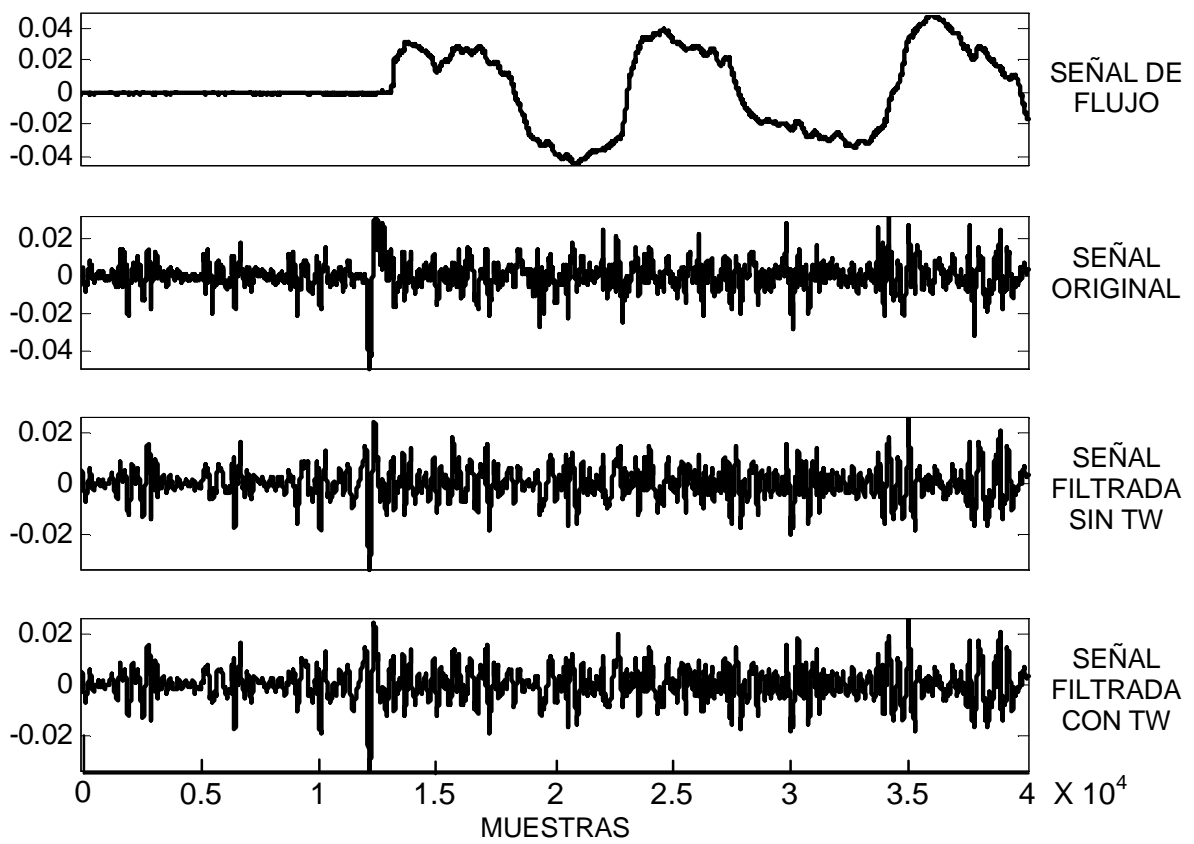

Figura 5.38 Señal obtenida de la zona de auscultación de la válvula aórtica y sus señales filtradas a flujo de $0.5 \mathrm{~L} / \mathrm{seg}$. 

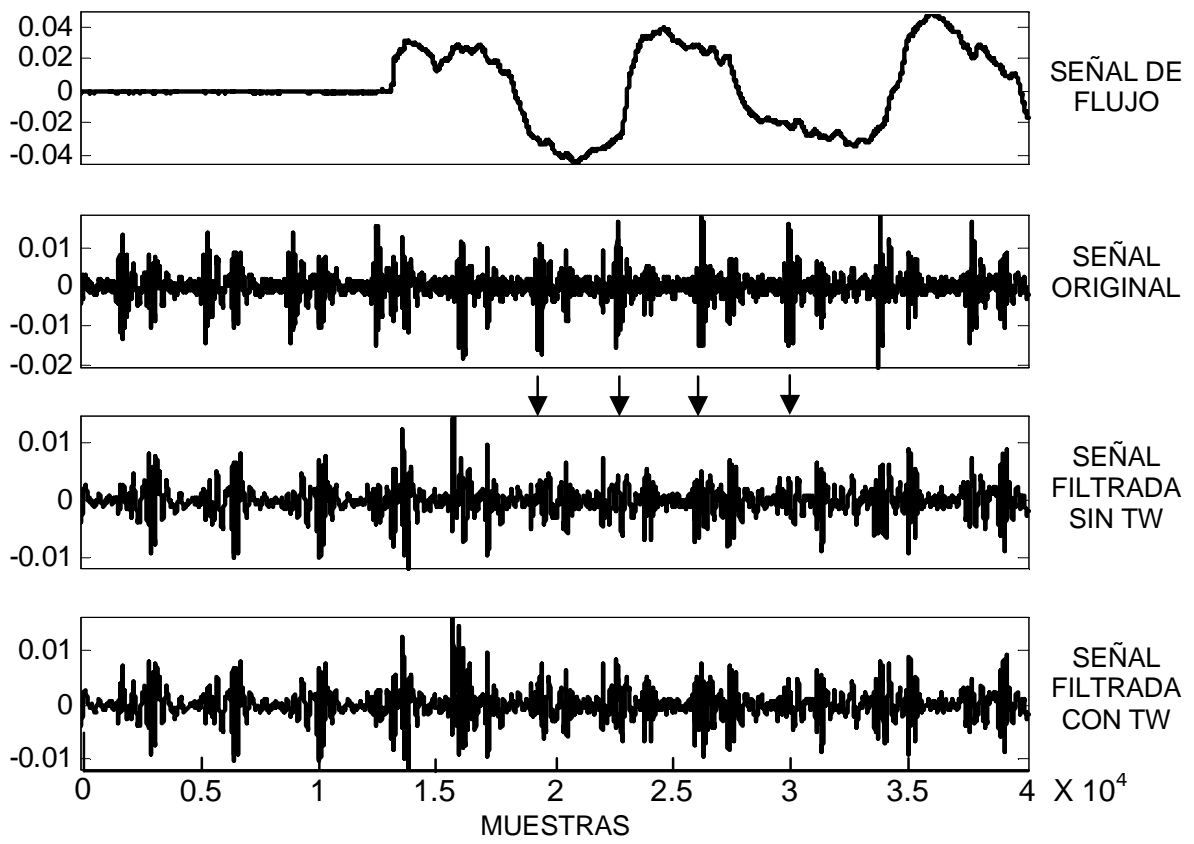

Figura 5.39 Señal obtenida de la zona de auscultación precordial promedio y sus señales filtradas a $0.5 \mathrm{~L} / \mathrm{seg}$.
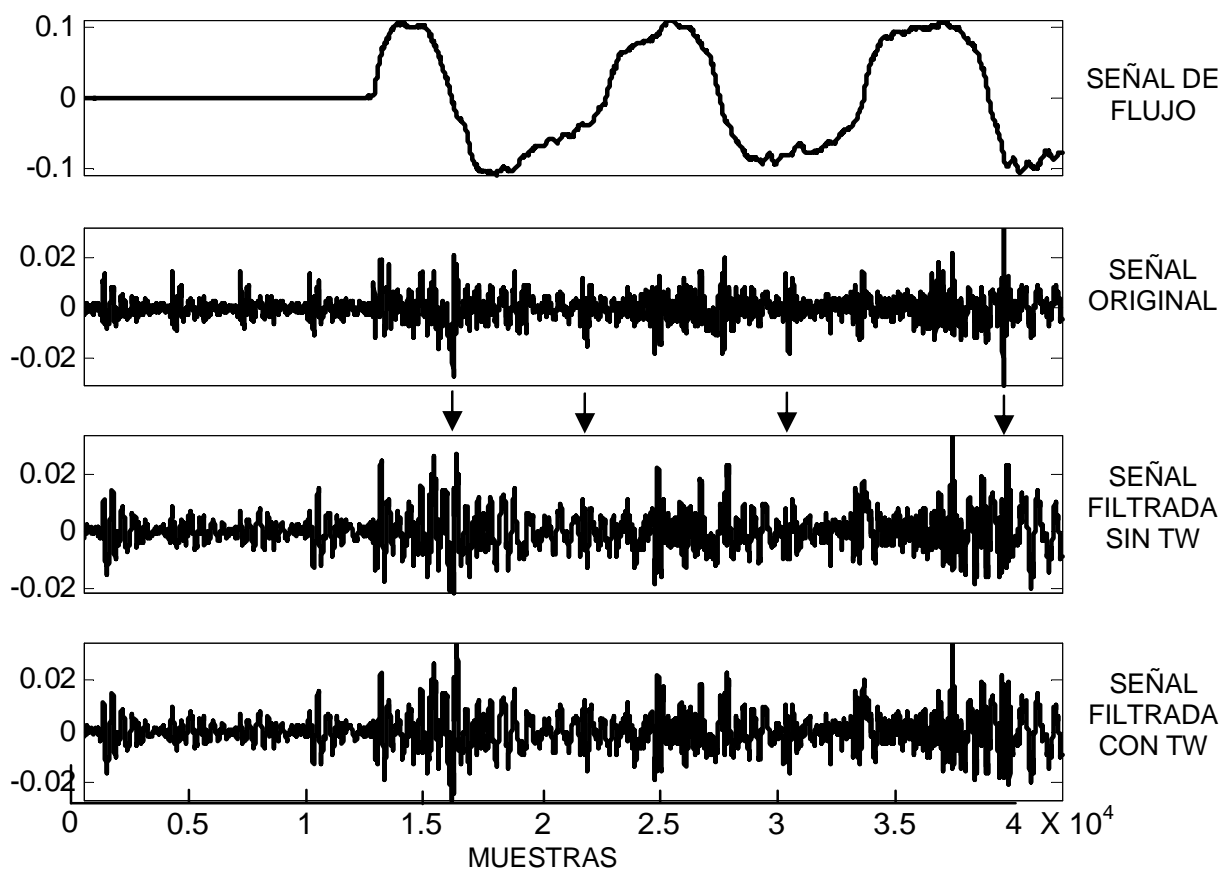

Figura 5.40 Señal obtenida de la línea axilar izquierda apical y sus señales filtradas a flujo de 2.0 L/seg. 

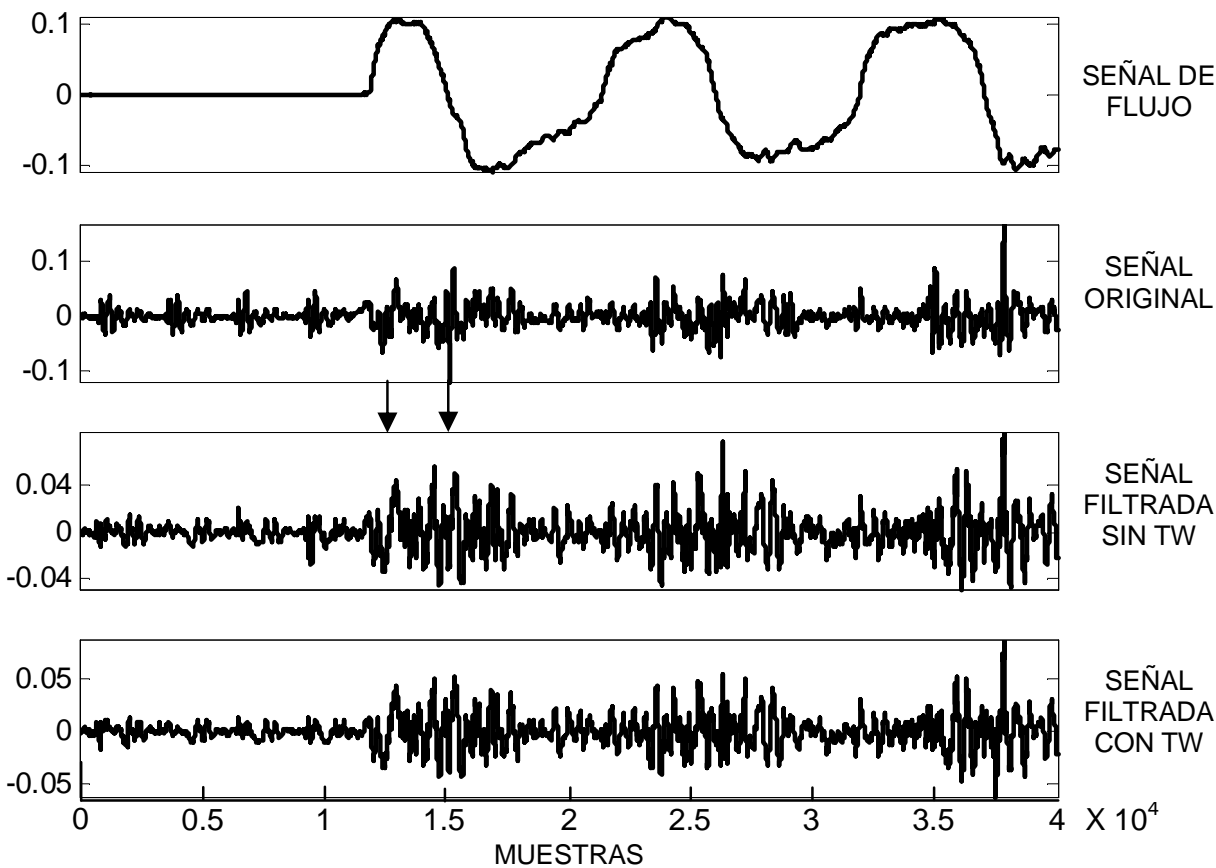

Figura 5.41 Señal obtenida de la línea axilar izquierda media y sus señales filtradas a flujo de $2.0 \mathrm{~L} / \mathrm{seg}$.
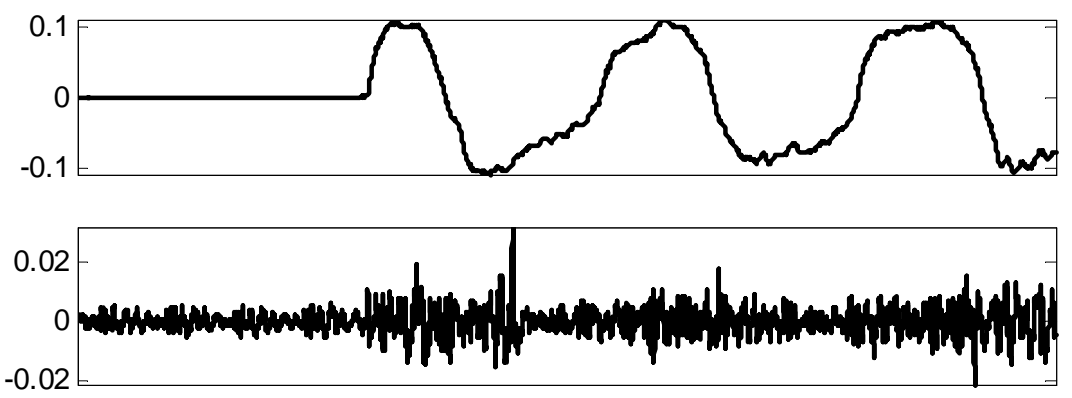

SEÑAL ORIGINAL
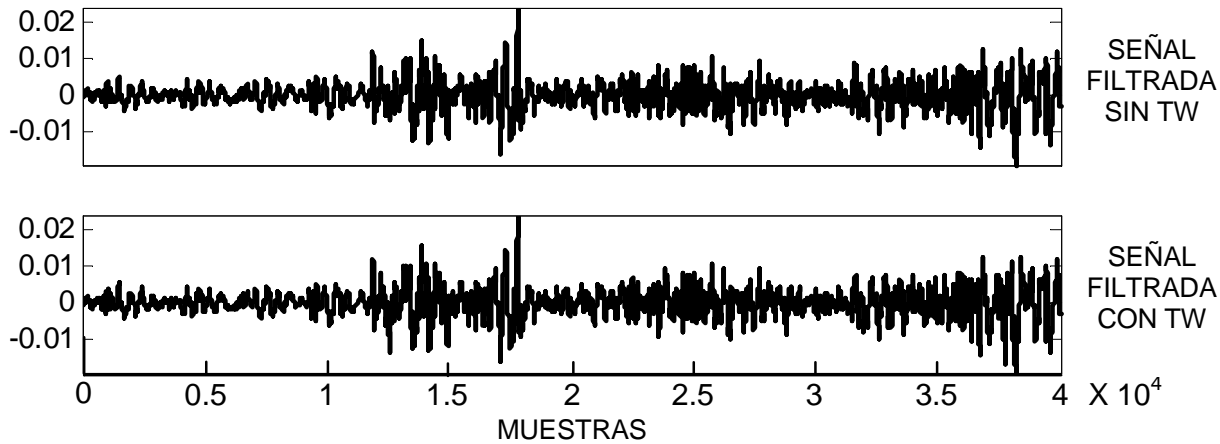

Figura 5.42 Señal obtenida de la línea axilar izquierda basal y sus señales filtradas a flujo de $2.0 \mathrm{~L} / \mathrm{seg}$. 

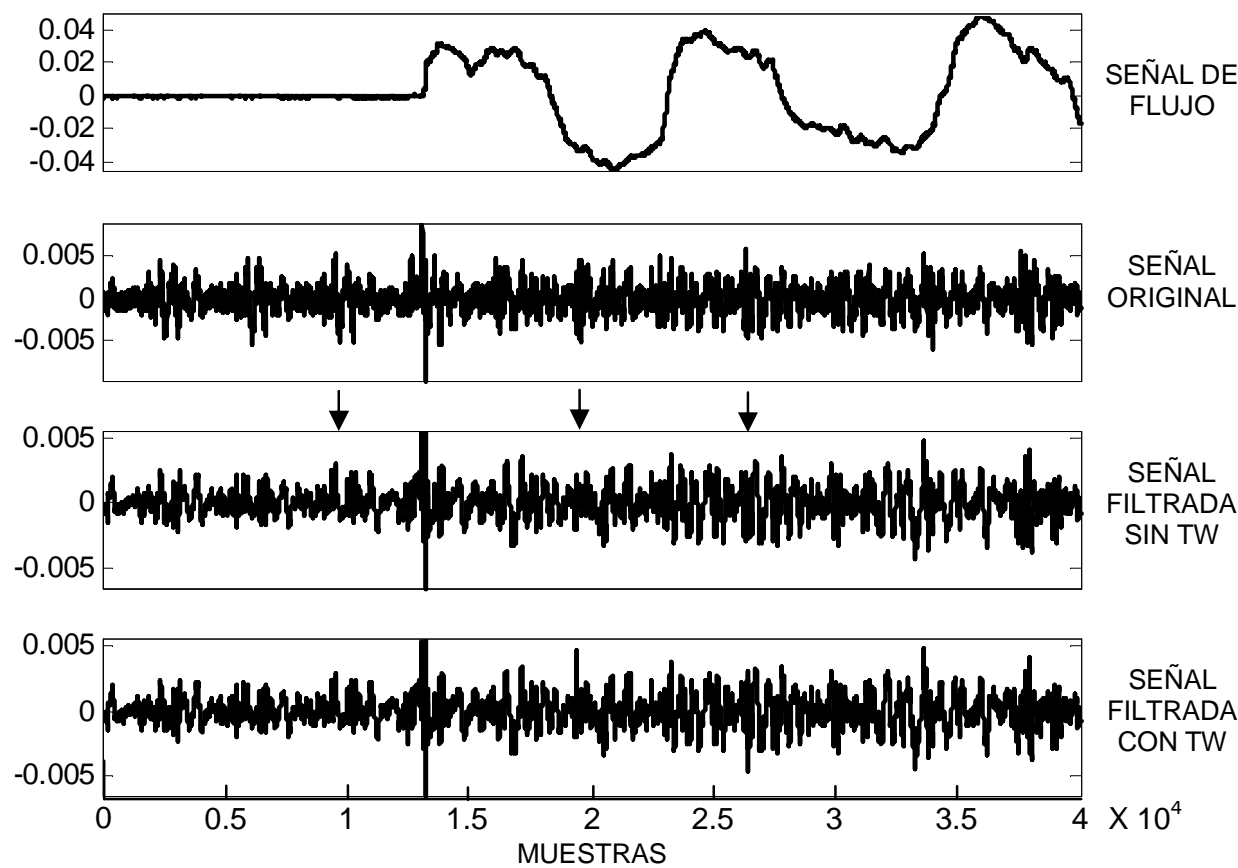

Figura 5.43 Señal obtenida de la línea axilar derecha apical y sus señales filtradas a flujo de 2.0 L/seg.
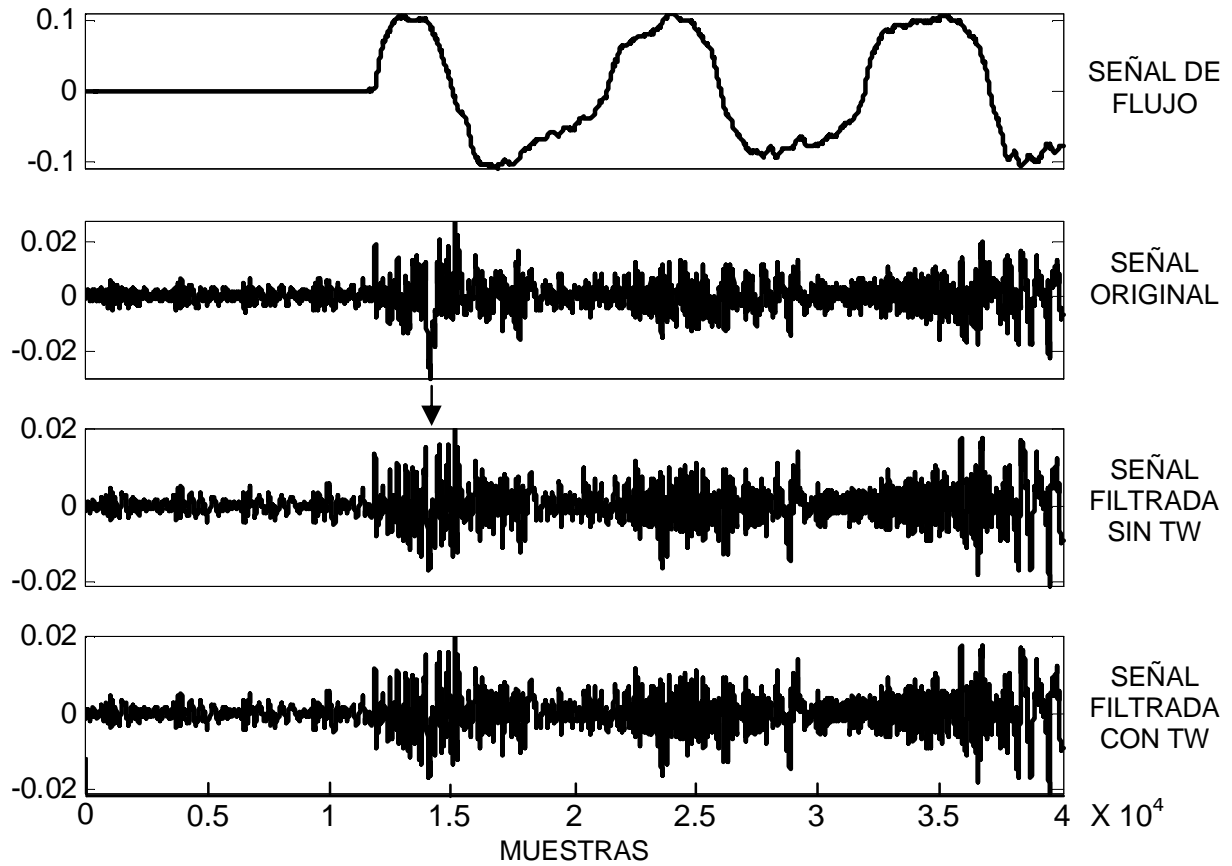

Figura 5.44 Señal obtenida de la línea axilar derecha media y sus señales filtradas a flujo de 2.0 L/seg. 

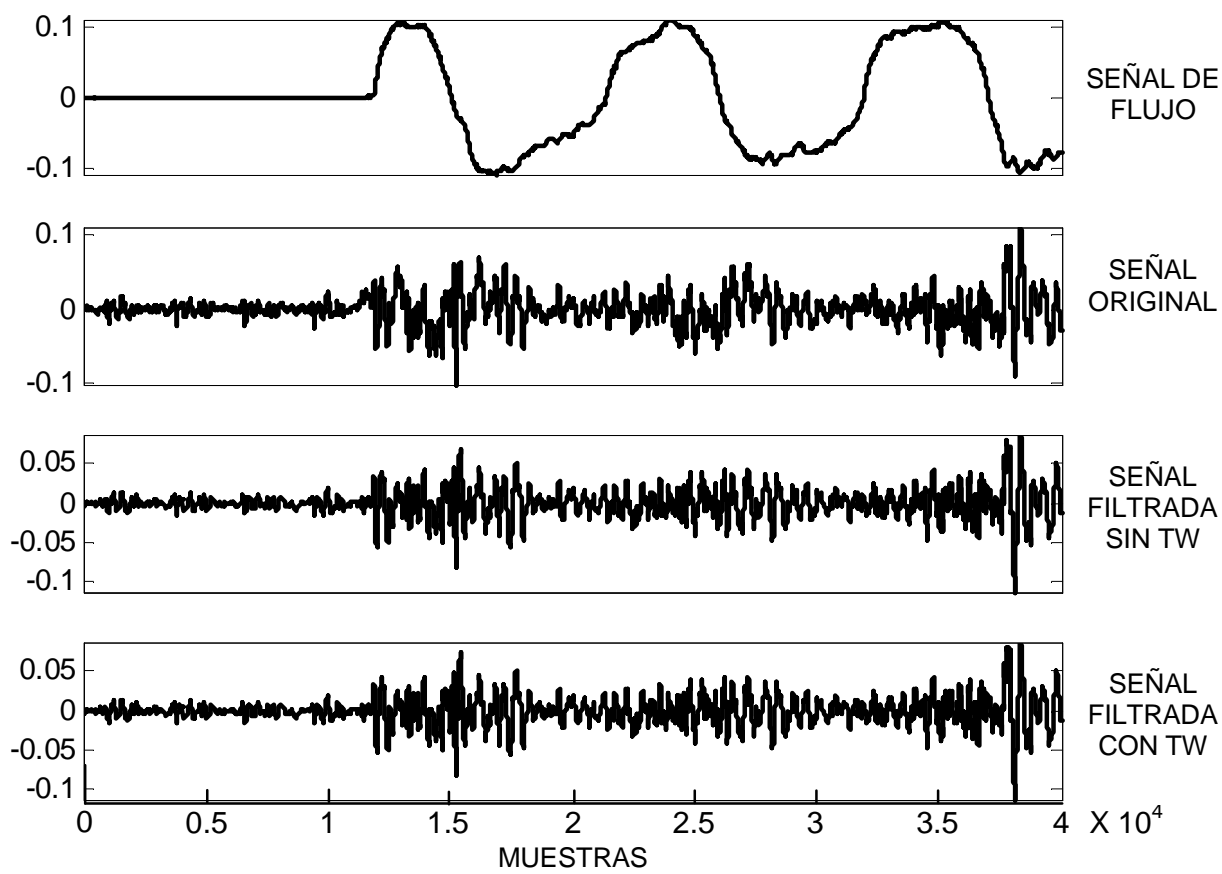

Figura 5.45 Señal obtenida de la línea axilar derecha basal y sus señales filtradas a flujo de $2.0 \mathrm{~L} / \mathrm{seg}$.
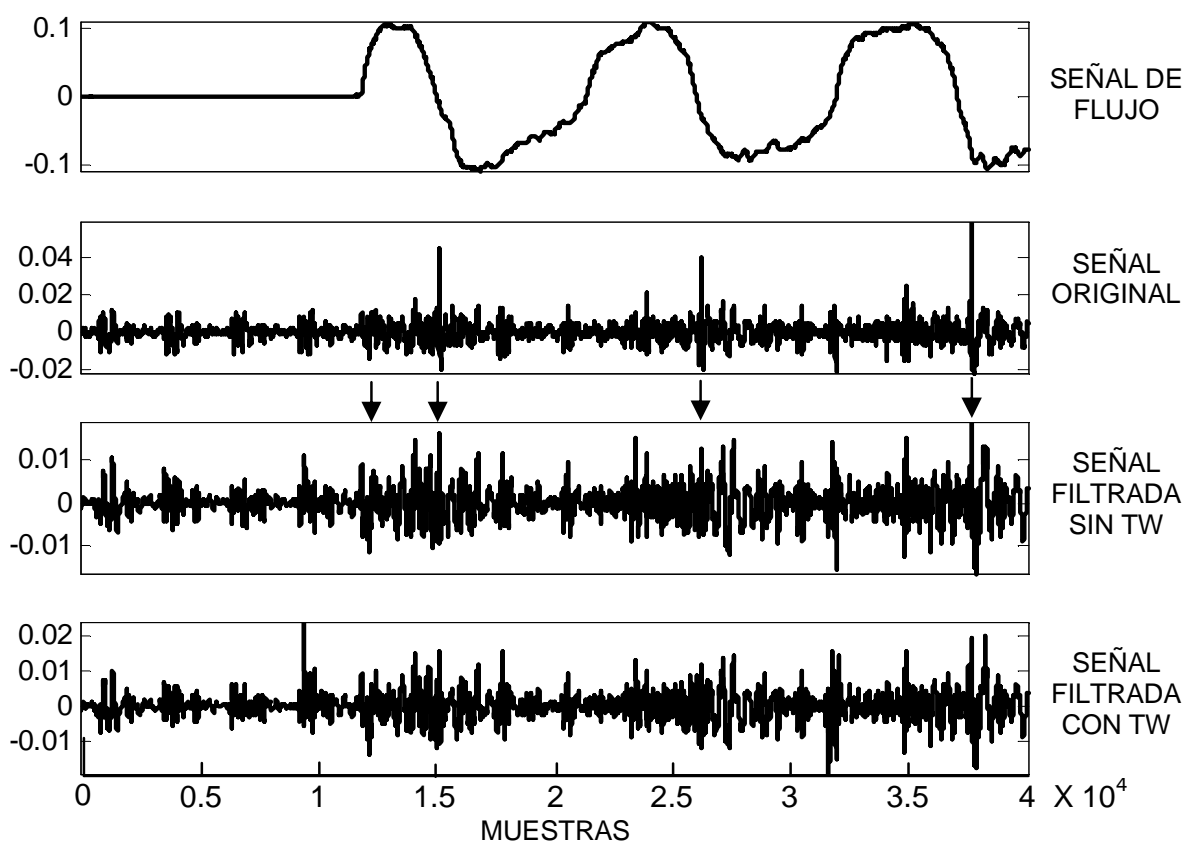

Figura 5.46 Señal obtenida de la zona de auscultación de la válvulas mitral y sus señales filtradas a flujo de $2.0 \mathrm{~L} / \mathrm{seg}$. 

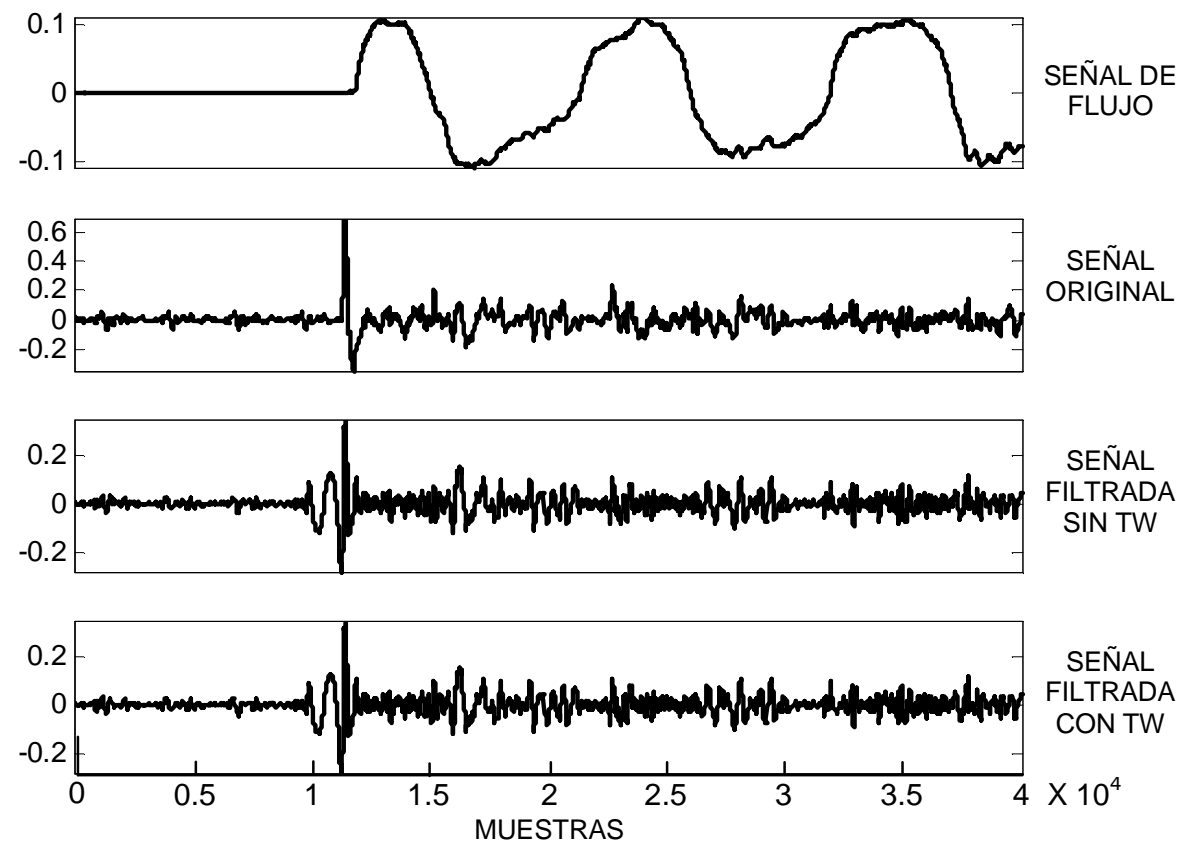

Figura 5.47 Señal obtenida de la zona de auscultación de la válvula pulmonar y sus señales filtradas a flujo de $2.0 \mathrm{~L} / \mathrm{seg}$.
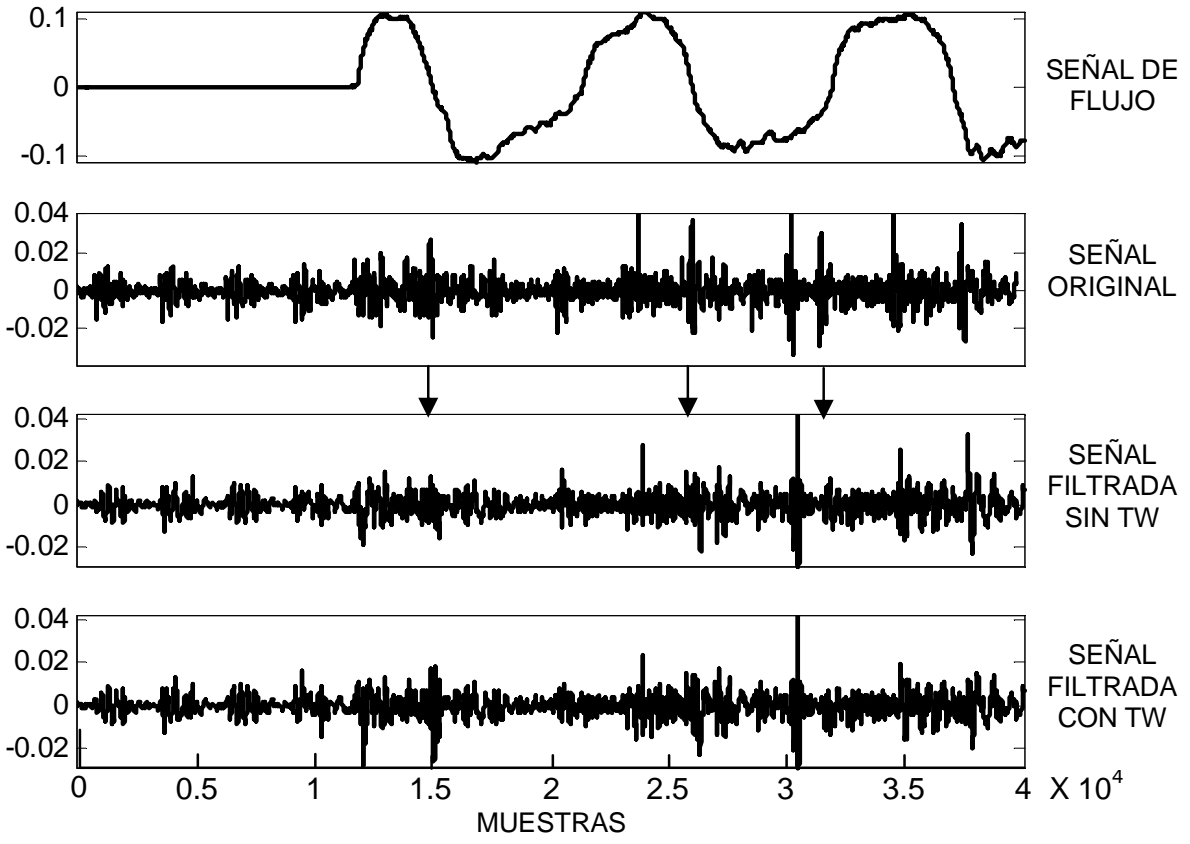

Figura 5.48 Señal obtenida de la zona de auscultación precordial promedio y sus señales filtradas a $2.0 \mathrm{~L} / \mathrm{seg}$. 


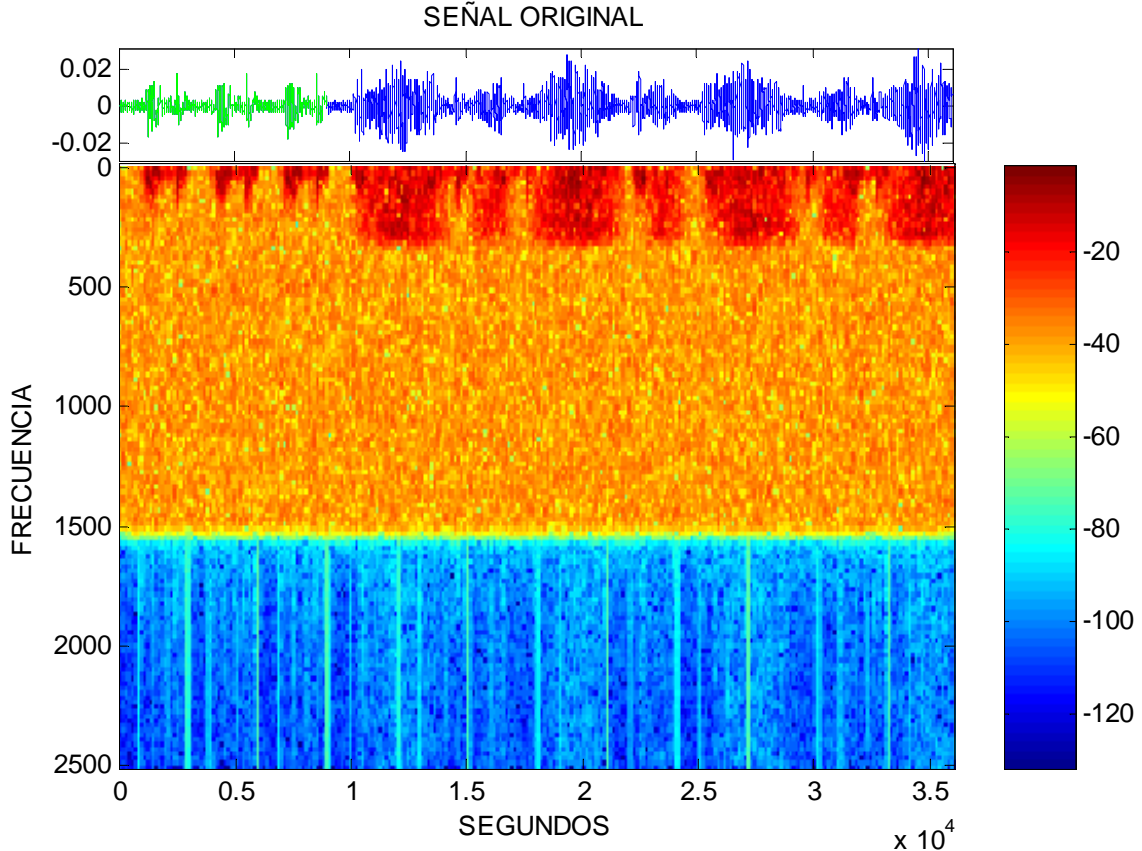

Figura 5.49 Representación tiempo-frecuencia de la señal acústica simulada con una relación señalruido de fondo de $20 \mathrm{~dB}$.

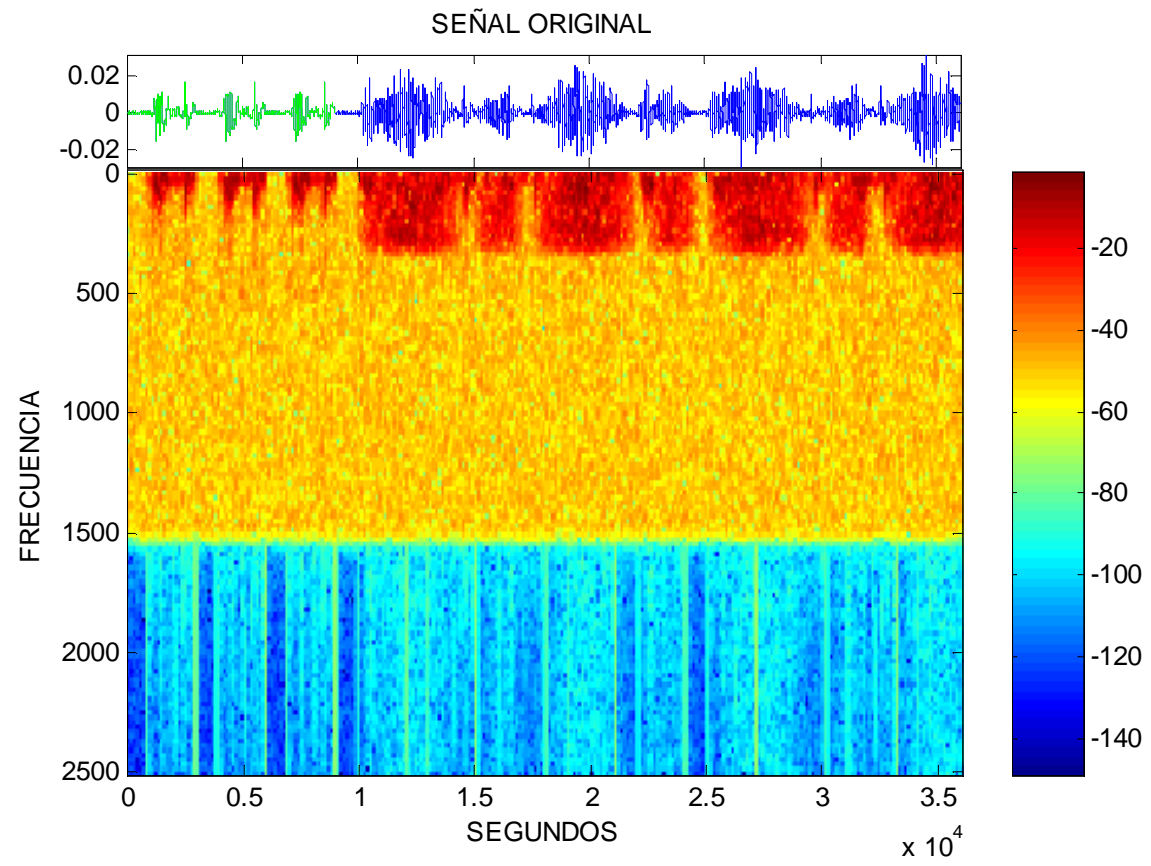

Figura 5.50 Representación tiempo-frecuencia de la señal acústica simulada con una relación señalruido de fondo de $50 \mathrm{~dB}$. 


\begin{tabular}{|c|c|c|c|c|c|c|c|c|c|c|c|c|}
\hline & \multicolumn{12}{|c|}{ RUIDO CARDIACOS } \\
\hline & \multicolumn{3}{|c|}{ ZONA DE APNEA } & \multicolumn{9}{|c|}{$\begin{array}{l}\text { ZONA DE RESPIRACION } \\
\end{array}$} \\
\hline & 1er & 20 & 3er & 40 & 50 & 60 & 70 & 80 & 90 & 100 & 110 & 120 \\
\hline SNR (dB) & 46.707 & 47.017 & 46.574 & 9.6671 & 0.87475 & 4.6944 & -13.445 & 19.16 & 2.2803 & -3.8554 & 9.0986 & -11.13 \\
\hline $\begin{array}{l}\text { BANDAS DE } \\
\text { FRECUENCIA }\end{array}$ & \multicolumn{12}{|c|}{ POTENCIAS DE RUIDOS CARDIACOS INSERTADOS } \\
\hline $0-300$ & 709.34 & 1083.4 & 907.19 & 577.17 & 732.64 & 495.17 & 865.02 & 649.94 & 831.52 & 546.7 & 889.35 & 745.23 \\
\hline $300-600$ & 33.386 & 43.93 & 37.768 & 28.481 & 28.207 & 22.588 & 30.511 & 28.392 & 29.322 & 22.755 & 33.688 & 30.804 \\
\hline $600-1200$ & 12.463 & 12.489 & 12.799 & 8.7555 & 8.8049 & 6.8559 & 9.8289 & 9.244 & 7.6754 & 7.1706 & 8.5358 & 8.6933 \\
\hline $\begin{array}{l}\text { BANDAS DE } \\
\text { FRECUENCIA }\end{array}$ & \multicolumn{12}{|c|}{ POTENCIAS DE RUIDOS CARDIACOS MAS RUIDO DE FONDO } \\
\hline $0-300$ & 724.9 & 1093.2 & 915.65 & 588.89 & 746.94 & 514.58 & 878.44 & 658.41 & 845.17 & 556.58 & 900.45 & 754.56 \\
\hline $300-600$ & 57.52 & 70.671 & 69.248 & 45.756 & 56.762 & 46.309 & 59.693 & 40.746 & 54.052 & 47.189 & 68.627 & 52.247 \\
\hline $600-1200$ & 142.59 & 152.41 & 158.79 & 112.08 & 133.83 & 125.97 & 154.55 & 105.32 & 141.54 & 122.39 & 168.4 & 110.46 \\
\hline $\begin{array}{l}\text { BANDAS DE } \\
\text { FRECUENCIA }\end{array}$ & \multicolumn{12}{|c|}{ POTENCIAS DE RUIDO CARDIACO ESTIMADO SIN TW } \\
\hline $0-300$ & 686.47 & 954.58 & 931.58 & 690.56 & 873.74 & 436.41 & 1035.6 & 718.44 & 931.31 & 522.73 & 979.22 & 1331.5 \\
\hline $300-600$ & 15.63 & 15.703 & 16.709 & 20.566 & 46.652 & 20.306 & 59.166 & 36.772 & 34.164 & 33.492 & 31.423 & 70.722 \\
\hline $600-1200$ & 3.8911 & 5.0768 & 4.1753 & 8.1697 & 16.02 & 12.822 & 20.354 & 10.366 & 13.25 & 12.695 & 14.123 & 22.166 \\
\hline $\begin{array}{l}\text { BANDAS DE } \\
\text { FRECUENCIA }\end{array}$ & \multicolumn{12}{|c|}{ POTENCIAS DE RUIDO CARDIACO ESTIMADO CON TW } \\
\hline $0-300$ & 672.61 & 927.84 & 912.8 & 734.7 & 898.6 & 461.51 & 1153.8 & 712.79 & 954.05 & 547.93 & 1023.7 & 1358.7 \\
\hline $300-600$ & 14.37 & 33.533 & 24.35 & 60.226 & 76.619 & 57.633 & 193.77 & 68.492 & 39.594 & 51.432 & 91.342 & 142.05 \\
\hline $600-1200$ & 6.0973 & 14.303 & 11.732 & 19.85 & 39.788 & 49.648 & 60.57 & 37.722 & 35.639 & 27.232 & 34.746 & 68.829 \\
\hline $\begin{array}{l}\text { BANDAS DE } \\
\text { FRECUENCIA }\end{array}$ & \multicolumn{12}{|c|}{ POTENCIAS DE SONIDO RESPIRATORIO } \\
\hline $0-300$ & 0 & 0 & 0 & 986.47 & 775.53 & 455.93 & 3104.1 & 471.81 & 1297.1 & 1047 & 575.81 & 2656 \\
\hline $300-600$ & 0 & 0 & 0 & 18.416 & 11.606 & 8.5064 & 63.179 & 6.7259 & 23.774 & 20.399 & 13.553 & 33.183 \\
\hline $600-1200$ & 0 & 0 & 0 & 0.49568 & 0.37503 & 0.2103 & 1.4741 & 0.23415 & 0.58275 & 0.48234 & 0.2937 & 1.1126 \\
\hline $\begin{array}{l}\text { BANDAS DE } \\
\text { FRECUENCIA }\end{array}$ & \multicolumn{12}{|c|}{ POTENCIAS DE SONIDO RESPIRATORIO MAS RUIDO DE FONDO } \\
\hline $0-300$ & 55.379 & 52.875 & 57.195 & 987.23 & 775.89 & 467.79 & 3102.5 & 475.18 & 1297.4 & 1046 & 594.55 & 2650.8 \\
\hline $300-600$ & 46.514 & 55.459 & 55.129 & 49.853 & 53.99 & 44.647 & 102.63 & 41.288 & 64.888 & 56.166 & 66.03 & 65.39 \\
\hline $600-1200$ & 142.66 & 150.81 & 157.6 & 112.22 & 133.11 & 124.65 & 153.93 & 104.1 & 140.39 & 122.4 & 168.91 & 109.33 \\
\hline $\begin{array}{l}\text { BANDAS DE } \\
\text { FRECUENCIA }\end{array}$ & \multicolumn{12}{|c|}{ POTENCIAS DE SONIDO RESPIRATORIO MAS RUIDO DE FONDO MAS RUIDO CARDIACO } \\
\hline $0-300$ & 724.9 & 1093.2 & 915.65 & 1299.5 & 1242.3 & 781.46 & 3511.7 & 1036.4 & 1892.1 & 1163.4 & 1279.5 & 2877.1 \\
\hline $300-600$ & 57.52 & 70.671 & 69.248 & 58.329 & 64.098 & 50.527 & 109.54 & 45.392 & 71.665 & 63 & 79.007 & 74.671 \\
\hline $600-1200$ & 142.59 & 152.41 & 158.79 & 112.02 & 133.83 & 125.97 & 154.51 & 105.32 & 141.48 & 122.33 & 168.42 & 110.4 \\
\hline $\begin{array}{l}\text { BANDAS DE } \\
\text { FRECUENCIA }\end{array}$ & \multicolumn{12}{|c|}{ POTENCIAS DE LAS SEÑALES DE SONIDO RESPIRATORIO ESTIMADO SIN TW } \\
\hline $0-300$ & 215.58 & 444.37 & 243.83 & 573.41 & 497.48 & 443.74 & 1159.2 & 503.51 & 699.68 & 474.13 & 367.51 & 765.59 \\
\hline $300-600$ & 29.652 & 27.27 & 27.702 & 26.276 & 52.836 & 25.651 & 60.247 & 38.193 & 35.578 & 40.396 & 36.161 & 69.787 \\
\hline $600-1200$ & 5.2874 & 6.4287 & 5.9015 & 8.48866 & 16.662 & 13.13 & 20.453 & 10.472 & 13.57 & 12.949 & 14.261 & 22.261 \\
\hline $\begin{array}{l}\text { BANDAS DE } \\
\text { FRECUENCIA }\end{array}$ & \multicolumn{12}{|c|}{ POTENCIAS DE LAS SEÑALES DE SONIDO RESPIRATORIO ESTIMADO SIN TW MAS LOS PRIMERO IMFS } \\
\hline $0-300$ & 225.4 & 452.64 & 249.9 & 929.6 & 775.43 & 572.43 & 3069.3 & 650.26 & 1297.6 & 901.53 & 588.77 & 2152.9 \\
\hline $300-600$ & 61.65 & 69.452 & 69.703 & 63.026 & 90.768 & 54.704 & 138.74 & 62.626 & 80.911 & 75.548 & 90.984 & 110.45 \\
\hline $600-1200$ & 142.56 & 152.33 & 158.82 & 112.5 & 136.11 & 127.62 & 155.99 & 106.33 & 140.99 & 123.36 & 169.63 & 116.41 \\
\hline $\begin{array}{l}\text { BANDAS DE } \\
\text { FRECUENCIA }\end{array}$ & \multicolumn{12}{|c|}{ POTENCIAS DE LAS SEÑALES DE SONIDO RESPIRATORIO ESTIMADO CON TW } \\
\hline $0-300$ & 154.2 & 366.74 & 227.04 & 553.88 & 583.37 & 486.88 & 1396.1 & 424.98 & 710.83 & 557.11 & 389.59 & 831.69 \\
\hline $300-600$ & 28.181 & 36.575 & 34.737 & 63.609 & 81.18 & 62.394 & 195.16 & 70.035 & 39.385 & 57.619 & 95.664 & 141.67 \\
\hline $600-1200$ & 7.5214 & 15.128 & 12.632 & 19.978 & 40.253 & 49.908 & 60.662 & 37.996 & 35.786 & 27.57 & 34.772 & 68.961 \\
\hline $\begin{array}{l}\text { BANDAS DE } \\
\text { FRECUENCIA }\end{array}$ & \multicolumn{12}{|c|}{ POTENCIAS DE LAS SEÑALES DE SONIDO RESPIRATORIO ESTIMADO CON TW MAS LOS PRIMERO IMFS } \\
\hline $0-300$ & 161.48 & 368.23 & 230.12 & 911.45 & 869.38 & 624.46 & 3321 & 568.99 & 1296 & 950.47 & 572.68 & 2087 \\
\hline $300-600$ & 58.454 & 73.838 & 70.779 & 91.501 & 115.15 & 84.544 & 245.7 & 82.782 & 88.551 & 94.088 & 132.85 & 167.15 \\
\hline $600-1200$ & 143.31 & 153.91 & 160.06 & 116.77 & 147.84 & 148.34 & 170.67 & 116.66 & 150.01 & 124.36 & 176.67 & 139.32 \\
\hline
\end{tabular}

Tabla 5. 1 Potencias obtenidas de señal simulada con una relación ruido cardiaco a ruido de fondo de $50 \mathrm{~dB}$. 


\begin{tabular}{|c|c|c|c|c|c|c|c|c|c|c|c|c|}
\hline & \multicolumn{12}{|c|}{ RUIDO CARDIACOS } \\
\hline & \multicolumn{3}{|c|}{ ZONA DE APNEA } & \multicolumn{9}{|c|}{ ZONA DE RESPIRACION } \\
\hline & 1er & 20 & 3er & 40 & 50 & 60 & 70 & 80 & 90 & 100 & 110 & 120 \\
\hline \multirow{2}{*}{$\begin{array}{c}\text { SNR (db) } \\
\text { BANDAS DE } \\
\text { FRECUENCIA }\end{array}$} & 46.707 & 47.017 & 46.574 & 9.6671 & 0.87475 & 4.6944 & -13.445 & 19.16 & 2.2803 & -3.8554 & 9.0986 & -11.13 \\
\hline & \multicolumn{12}{|c|}{ POTENCIAS DE RUIDOS CARDIACOS INSERTADOS } \\
\hline $0-300$ & 709.34 & 1083.4 & 907.19 & 577.17 & 732.64 & 495.17 & 865.02 & 649.94 & 831.52 & 546.7 & 889.35 & 745.23 \\
\hline $300-600$ & 33.386 & 43.93 & 37.768 & 28.481 & 28.207 & 22.588 & 30.511 & 28.392 & 29.322 & 22.755 & 33.688 & 30.804 \\
\hline $600-1200$ & 12.463 & 12.489 & 12.799 & 8.7555 & 8.8049 & 6.8559 & 9.8289 & 9.244 & 7.6754 & 7.1706 & 8.5358 & 8.6933 \\
\hline $\begin{array}{l}\text { BANDAS DE } \\
\text { FRECUENCIA }\end{array}$ & \multicolumn{12}{|c|}{ POTENCIAS DE RUIDOS CARDIACOS MAS RUIDO DE FONDO } \\
\hline $0-300$ & 826.46 & 1179.8 & 1002.1 & 667.07 & 845.09 & 605.8 & 994.41 & 728.98 & 932.77 & 622.72 & 996.3 & 823.09 \\
\hline $300-600$ & 187.75 & 222.65 & 227.24 & 147.37 & 188.2 & 163.26 & 200.79 & 146.1 & 185.7 & 160.22 & 224.84 & 163.3 \\
\hline $600-1200$ & 569.41 & 603.89 & 630.8 & 447.87 & 532.73 & 499.55 & 615.93 & 417.02 & 562.52 & 489.36 & 674.58 & 437.92 \\
\hline $\begin{array}{l}\text { BANDAS DE } \\
\text { FRECUENCIA }\end{array}$ & \multicolumn{12}{|c|}{ POTENCIAS DE RUIDO CARDIACO ESTIMADO SIN TW } \\
\hline $0-300$ & 678.36 & 680.08 & 713.84 & 725.91 & 600.55 & 399.18 & 1455.9 & 657.08 & 444.77 & 0 & 825.92 & 1460.8 \\
\hline $300-600$ & 24.922 & 16.017 & 21.418 & 21.409 & 25.598 & 9.1174 & 139.12 & 18.855 & 15.021 & 0 & 18.986 & 164.96 \\
\hline $600-1200$ & 11.915 & 8.379 & 10.429 & 14.26 & 32.053 & 8.8471 & 87.261 & 10.637 & 4.4532 & 0 & 10.236 & 107.1 \\
\hline $\begin{array}{l}\text { BANDAS DE } \\
\text { FRECUENCIA }\end{array}$ & \multicolumn{12}{|c|}{ POTENCIAS DE RUIDO CARDIACO ESTIMADO CON TW } \\
\hline $0-300$ & 687.38 & 665.43 & 761.81 & 747.73 & 626.76 & 441.77 & 1420 & 644.38 & 405.4 & 0 & 817.72 & 1542.4 \\
\hline $300-600$ & 39.472 & 19.015 & 37.355 & 29.182 & 78.563 & 23.85 & 263.4 & 53.038 & 10.052 & 0 & 28.358 & 224.97 \\
\hline $600-1200$ & 9.6046 & 6.4972 & 5.9412 & 11.583 & 8.0645 & 3.4858 & 51.262 & 4.922 & 5.0266 & 0 & 5.6038 & 81.405 \\
\hline $\begin{array}{l}\text { BANDAS DE } \\
\text { FRECUENCIA }\end{array}$ & \multicolumn{12}{|c|}{ POTENCIAS DE SONIDO RESPIRATORIO } \\
\hline $0-300$ & 0 & 0 & 0 & 986.47 & 775.53 & 455.93 & 3104.1 & 471.81 & 1297.1 & 1047 & 575.81 & 2656 \\
\hline $300-600$ & 0 & 0 & 0 & 18.416 & 11.606 & 8.5064 & 63.179 & 6.7259 & 23.774 & 20.399 & 13.553 & 33.183 \\
\hline $600-1200$ & 0 & 0 & 0 & 0.49568 & 0.37503 & 0.2103 & 1.4741 & 0.23415 & 0.58275 & 0.48234 & 0.2937 & 1.1126 \\
\hline $\begin{array}{l}\text { BANDAS DE } \\
\text { FRECUENCIA }\end{array}$ & \multicolumn{12}{|c|}{ POTENCIAS DE SONIDO RESPIRATORIO MAS RUIDO DE FONDO } \\
\hline $0-300$ & 221.51 & 211.5 & 228.78 & 1000.5 & 811.07 & 526.89 & 3106.5 & 512.47 & 1311.7 & 1059.6 & 682.21 & 2639.9 \\
\hline $300-600$ & 186.06 & 221.84 & 220.52 & 152.74 & 189.37 & 161.59 & 234 & 151.48 & 198.47 & 169.44 & 227.73 & 173.08 \\
\hline $600-1200$ & 570.63 & 603.23 & 630.39 & 449.09 & 532.45 & 498.61 & 615.82 & 416.39 & 561.77 & 489.77 & 675.6 & 437.51 \\
\hline $\begin{array}{l}\text { BANDAS DE } \\
\text { FRECUENCIA }\end{array}$ & \multicolumn{12}{|c|}{ POTENCIAS DE SONIDO RESPIRATORIO MAS RUIDO DE FONDO MAS RUIDO CARDIACOS } \\
\hline $0-300$ & 826.46 & 1179.8 & 1002.1 & 1325.6 & 1262.5 & 834.43 & 3522.8 & 1055.3 & 1910.4 & 1166.3 & 1343.5 & 2868.2 \\
\hline $300-600$ & 187.75 & 222.65 & 227.24 & 154.56 & 193.06 & 164.2 & 236.91 & 148.72 & 198.36 & 173.16 & 233.8 & 177.61 \\
\hline $600-1200$ & 569.41 & 603.89 & 630.8 & 447.81 & 532.73 & 499.54 & 615.89 & 417.02 & 562.45 & 489.3 & 674.6 & 437.85 \\
\hline $\begin{array}{l}\text { BANDAS DE } \\
\text { FRECUENCIA }\end{array}$ & \multicolumn{12}{|c|}{ POTENCIAS DE LAS SEÑALES DE SONIDO RESPIRATORIO ESTIMADO SIN TW } \\
\hline $0-300$ & 346.44 & 779.79 & 656.73 & 599.33 & 758.32 & 467.24 & 1731 & 667.4 & 1504.6 & 872.59 & 858.7 & 1334.2 \\
\hline $300-600$ & 39.775 & 41.808 & 40.028 & 27.105 & 35.189 & 33.811 & 142.1 & 29.499 & 41.239 & 28.897 & 36.027 & 164.8 \\
\hline $600-1200$ & 11.091 & 8.1618 & 7.5493 & 11.691 & 9.1755 & 5.1151 & 51.397 & 6.0043 & 8.409 & 3.7829 & 7.6655 & 81.441 \\
\hline $\begin{array}{l}\text { BANDAS DE } \\
\text { FRECUENCIA }\end{array}$ & \multicolumn{12}{|c|}{ POTENCIAS DE LAS SEÑALES DE SONIDO RESPIRATORIO ESTIMADO SIN TW MAS LOS PRIMERO IMFS } \\
\hline $0-300$ & 364.92 & 799.75 & 669.45 & 939.17 & 1021 & 574.08 & 3141.9 & 804.62 & 1841.4 & 1167 & 1036.7 & 2319.3 \\
\hline $300-600$ & 193.51 & 222.73 & 225.38 & 153.06 & 192.46 & 164.05 & 301.36 & 150.8 & 197.65 & 173.16 & 239.23 & 262.77 \\
\hline $600-1200$ & 570.05 & 603.78 & 629.88 & 449.17 & 532.8 & 499.51 & 625.08 & 417.49 & 562.33 & 489.3 & 674.66 & 450.21 \\
\hline $\begin{array}{l}\text { BANDAS DE } \\
\text { FRECUENCIA }\end{array}$ & \multicolumn{12}{|c|}{ POTENCIAS DE LAS SEÑALES DE SONIDO RESPIRATORIO ESTIMADO CON TW } \\
\hline 0-300 & 338.86 & 765.3 & 713.76 & 565.07 & 782.34 & 569.99 & 1618.4 & 648.67 & 1512.2 & 872.59 & 761.76 & 1398.8 \\
\hline $300-600$ & 48.345 & 42.552 & 54.39 & 36.757 & 84.877 & 46.613 & 264.04 & 59.784 & 38.759 & 28.897 & 42.681 & 223.64 \\
\hline $600-1200$ & 13.323 & 9.8189 & 11.477 & 14.224 & 32.452 & 10.251 & 87.398 & 11.089 & 7.7686 & 3.7829 & 11.718 & 107.1 \\
\hline $\begin{array}{l}\text { BANDAS DE } \\
\text { FRECUENCIA }\end{array}$ & \multicolumn{12}{|c|}{ POTENCIAS DE LAS SEÑALES DE SONIDO RESPIRATORIO ESTIMADO CON TW MAS LOS PRIMERO IMFS } \\
\hline $0-300$ & 359.76 & 780.82 & 732.79 & 928.73 & 1024 & 671.93 & 2938.2 & 784.15 & 1852.7 & 1167 & 927.74 & 2257.9 \\
\hline $300-600$ & 198.48 & 223.05 & 231.33 & 156.89 & 210 & 166 & 378.69 & 173.81 & 197.7 & 173.16 & 232.43 & 304.68 \\
\hline $600-1200$ & 570.25 & 603.56 & 629.36 & 449.11 & 530.95 & 500.2 & 636.05 & 419.11 & 561.93 & 489.3 & 675.02 & 462.05 \\
\hline
\end{tabular}

Tabla 5. 2 Potencias obtenidas de señal simulada con una relación señal respiratoria-ruido cardiaco de $20 \mathrm{~dB}$. 
Tabla 5. 3 Tablas de Potencias calculadas en el domino original en los diferentes puntos de adquisición para un flujo de $0.5 \mathrm{~L} / \mathrm{seg}$.

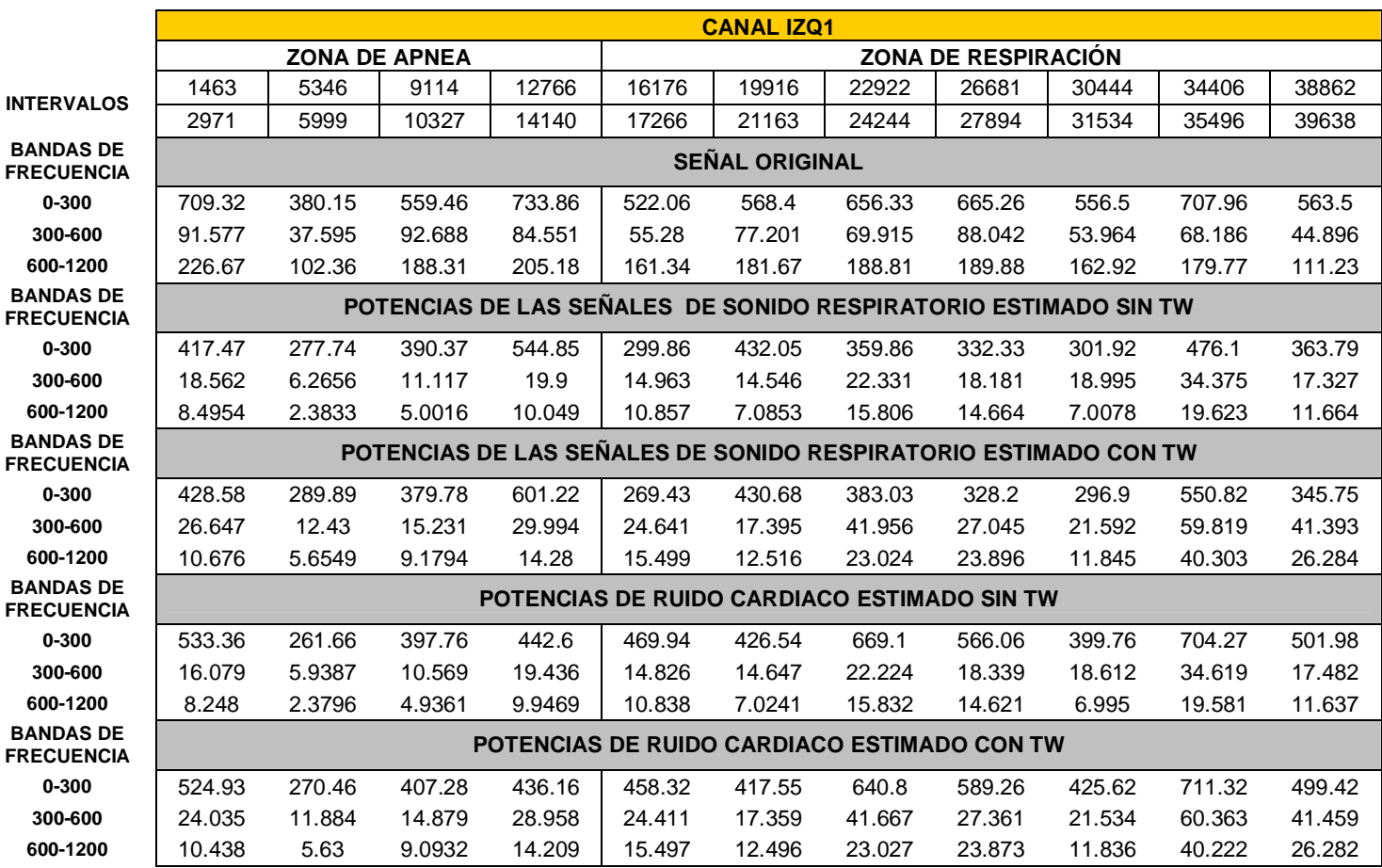

Tabla 5. 3.a. Potencias calculadas de la señal en el dominio original para la línea axilar izquierda apical a un flujo de $0.5 \mathrm{~L} / \mathrm{seg}$.

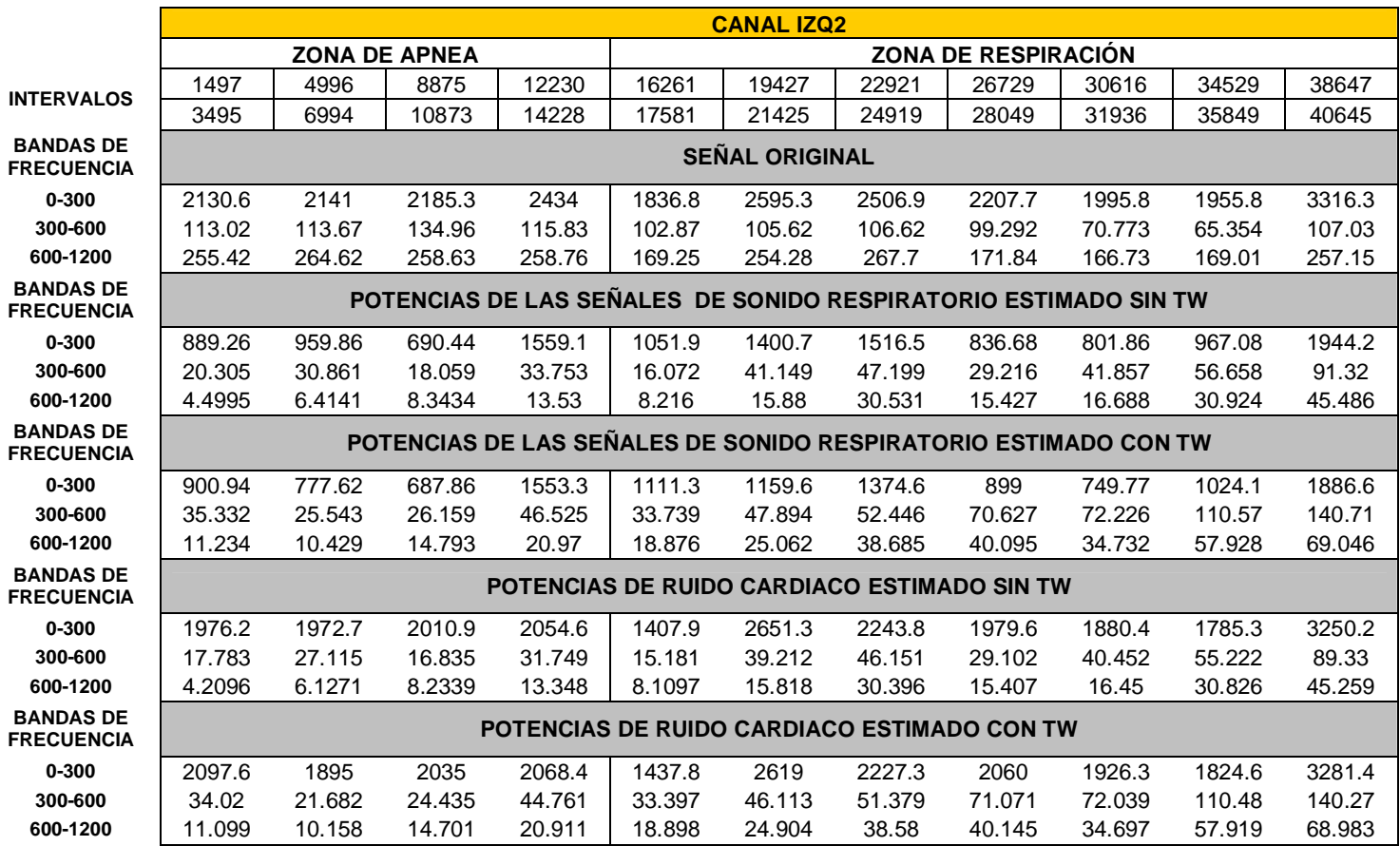

Tabla 5. 3.b. Potencias calculadas de la señal en el dominio original para la línea axilar izquierda media a un flujo de $0.5 \mathrm{~L} / \mathrm{seg}$. 


\begin{tabular}{|c|c|c|c|c|c|c|c|c|c|c|c|}
\hline \multirow{4}{*}{ INTERVALOS } & \multicolumn{11}{|c|}{ CANAL IZQ3 } \\
\hline & \multicolumn{4}{|c|}{ ZONA DE APNEA } & \multicolumn{7}{|c|}{ ZONA DE RESPIRACIÓN } \\
\hline & 1439 & 5041 & 8679 & 12155 & 16286 & 19746 & 23244 & 26770 & 30583 & 34669 & 38594 \\
\hline & 4194 & 7796 & 11434 & 14910 & 19266 & 21141 & 24551 & 28007 & 31820 & 35906 & 39831 \\
\hline $\begin{array}{l}\text { BANDAS DE } \\
\text { FRECUENCIA }\end{array}$ & \multicolumn{11}{|c|}{ SEÑAL ORIGINAL } \\
\hline $0-300$ & 1042.6 & 1047.4 & 1030.8 & 1132.3 & 1134.5 & 799.76 & 966.83 & 748.67 & 802.36 & 822.3 & 794.82 \\
\hline $300-600$ & 163.78 & 163.45 & 177.03 & 153.22 & 211.83 & 84.477 & 64.708 & 82.016 & 76.734 & 74.569 & 65.527 \\
\hline $600-1200$ & 403.78 & 400.37 & 403.48 & 399.86 & 466.12 & 193.88 & 210.82 & 201.21 & 192.43 & 198.43 & 187.6 \\
\hline $\begin{array}{l}\text { BANDAS DE } \\
\text { FRECUENCIA }\end{array}$ & \multicolumn{11}{|c|}{ POTENCIAS DE LAS SEÑALES DE SONIDO RESPIRATORIO ESTIMADO SIN TW } \\
\hline $0-300$ & 363.53 & 489.42 & 460.42 & 618.2 & 570.7 & 557.14 & 533.35 & 428.66 & 424.38 & 393.74 & 519.84 \\
\hline $300-600$ & 3.9957 & 21.619 & 9.3275 & 29.759 & 24.58 & 120.44 & 105.82 & 54.508 & 24.053 & 33.802 & 31.683 \\
\hline $600-1200$ & 1.6686 & 9.006 & 3.1663 & 13.663 & 9.605 & 54.431 & 47.676 & 25.638 & 9.8597 & 16.2 & 13.499 \\
\hline $\begin{array}{l}\text { BANDAS DE } \\
\text { FRECUENCIA }\end{array}$ & \multicolumn{11}{|c|}{ POTENCIAS DE LAS SEÑALES DE SONIDO RESPIRATORIO ESTIMADO CON TW } \\
\hline $0-300$ & 365.32 & 515.47 & 443.64 & 658.45 & 570.62 & 551.37 & 668.45 & 477.02 & 451.99 & 402.37 & 498.38 \\
\hline $300-600$ & 6.2424 & 44.394 & 10.9 & 93.448 & 54.649 & 88.818 & 139.18 & 112.29 & 36.685 & 47.938 & 39.049 \\
\hline $600-1200$ & 2.1559 & 23.944 & 4.2871 & 48.503 & 17.869 & 33.213 & 48.945 & 35.762 & 15.206 & 21.415 & 17.858 \\
\hline $\begin{array}{l}\text { BANDAS DE } \\
\text { FRECUENCIA }\end{array}$ & \multicolumn{11}{|c|}{ POTENCIAS DE RUIDO CARDIACO ESTIMADO SIN TW } \\
\hline 0-300 & 806.97 & 872.97 & 815.25 & 811.8 & 863.36 & 936.5 & 815.4 & 602.85 & 669.64 & 755.78 & 741.3 \\
\hline $300-600$ & 3.6964 & 21.477 & 9.0917 & 29.627 & 24.415 & & 105.86 & & & 33.825 & 31.664 \\
\hline $600-1200$ & 1.6541 & 8.9921 & 3.1566 & 13.669 & 9.5758 & 54.427 & 47.676 & 25.642 & 9.8565 & 16.197 & 13.5 \\
\hline $\begin{array}{l}\text { BANDAS DE } \\
\text { FRECUENCIA }\end{array}$ & \multicolumn{11}{|c|}{ POTENCIAS DE RUIDO CARDIACO ESTIMADO CON TW } \\
\hline $0-300$ & 812.37 & 940.65 & 820.22 & 880.06 & 872.49 & 914.48 & 968.15 & 655.15 & 689.36 & 743.13 & 744.63 \\
\hline $300-600$ & 5.9583 & 44.185 & 10.739 & 93.407 & 54.569 & 88.826 & 139.18 & 112.33 & 36.647 & 47.884 & 39.129 \\
\hline $600-1200$ & 2.1347 & 23.932 & 4.2789 & 48.492 & 17.871 & 33.209 & 48.946 & 35.757 & 15.212 & 21.419 & 17.854 \\
\hline
\end{tabular}

Tabla 5. 3.c. Potencias calculadas de la señal en el dominio original para la línea axilar izquierda basal a un flujo de $0.5 \mathrm{~L} / \mathrm{seg}$.

\begin{tabular}{|c|c|c|c|c|c|c|c|c|c|c|c|}
\hline \multirow{4}{*}{ INTERVALOS } & \multicolumn{11}{|c|}{ DER1 } \\
\hline & \multicolumn{4}{|c|}{ ZONA DE APNEA } & \multicolumn{7}{|c|}{ ZONA DE RESPIRACIÓN } \\
\hline & 1689 & 5720 & 9111 & 12753 & 0 & 19960 & 0 & 0 & 31011 & 0 & 38729 \\
\hline & 3991 & 7654 & 11362 & 15028 & 0 & 22208 & 0 & 0 & 32945 & 0 & 40575 \\
\hline $\begin{array}{l}\text { BANDAS DE } \\
\text { FRECUENCIA }\end{array}$ & \multicolumn{11}{|c|}{ SEÑAL ORIGINAL } \\
\hline $0-300$ & 585.68 & 526.68 & 629.01 & 805.63 & 0 & 685.66 & 0 & 0 & 536.41 & 0 & 689.76 \\
\hline $300-600$ & 117.07 & 96.518 & 137.24 & 111.6 & 0 & 111.71 & 0 & 0 & 86.556 & 0 & 92.902 \\
\hline $600-1200$ & 275.88 & 238.34 & 304.74 & 293.69 & 0 & 283.92 & 0 & 0 & 246.73 & 0 & 238.93 \\
\hline $\begin{array}{l}\text { BANDAS DE } \\
\text { FRECUENCIA }\end{array}$ & \multicolumn{11}{|c|}{ POTENCIAS DE LAS SEÑALES DE SONIDO RESPIRATORIO ESTIMADO SIN TW } \\
\hline $0-300$ & 390.31 & 312.95 & 409.78 & 627.52 & 0 & 530.44 & 0 & 0 & 409.49 & 0 & 491.51 \\
\hline $300-600$ & 9.6003 & 6.6476 & 5.8064 & 9.9611 & 0 & 14.423 & 0 & 0 & 8.0617 & 0 & 14.056 \\
\hline $600-1200$ & 2.0809 & 1.0496 & 1.9931 & 5.4238 & 0 & 6.7432 & 0 & 0 & 5.1456 & 0 & 5.8115 \\
\hline $\begin{array}{l}\text { BANDAS DE } \\
\text { FRECUENCIA }\end{array}$ & \multicolumn{11}{|c|}{ POTENCIAS DE LAS SEÑALES DE SONIDO RESPIRATORIO ESTIMADO CON TW } \\
\hline $0-300$ & 388.87 & 312.34 & 410.18 & 626.15 & 0 & 578.62 & 0 & 0 & 420.62 & 0 & 461.47 \\
\hline $300-600$ & 10.266 & 6.7477 & 6.0285 & 15.368 & 0 & 15.146 & 0 & 0 & 10.712 & 0 & 14.292 \\
\hline $600-1200$ & 2.6458 & 1.0039 & 2.3472 & 7.5909 & 0 & 7.6135 & 0 & 0 & 5.7263 & 0 & 8.4741 \\
\hline $\begin{array}{l}\text { BANDAS DE } \\
\text { FRECUENCIA }\end{array}$ & \multicolumn{11}{|c|}{ POTENCIAS DE RUIDO CARDIACO ESTIMADO SIN TW } \\
\hline $0-300$ & 422.78 & 400.94 & 464.59 & 544.77 & 0 & 518.01 & 0 & 0 & 413.88 & 0 & 582.8 \\
\hline $300-600$ & 2.7836 & 1.5338 & 2.921 & 7.7916 & 0 & 11.044 & 0 & 0 & 5.7571 & 0 & 11.697 \\
\hline $600-1200$ & 1.2873 & 0.44377 & 1.6677 & 5.1357 & 0 & 6.3701 & 0 & 0 & 4.8711 & 0 & 5.4565 \\
\hline $\begin{array}{l}\text { BANDAS DE } \\
\text { FRECUENCIA }\end{array}$ & \multicolumn{11}{|c|}{ POTENCIAS DE RUIDO CARDIACO ESTIMADO CON TW } \\
\hline $0-300$ & 425.75 & 400.29 & 467.86 & 544.64 & 0 & 551.36 & 0 & 0 & 422.75 & 0 & 577.6 \\
\hline $300-600$ & 4.2364 & 1.4199 & 3.7995 & 13.641 & 0 & 10.82 & 0 & 0 & 9.1725 & 0 & 12.241 \\
\hline $600-1200$ & 1.9804 & 0.37213 & 2.1375 & 7.412 & 0 & 7.2622 & 0 & 0 & 5.6286 & 0 & 8.297 \\
\hline
\end{tabular}

Tabla 5. 3.d. Potencias calculadas de la señal en el dominio original para la línea axilar derecha apical a un flujo de $0.5 \mathrm{~L} / \mathrm{seg}$. 


\begin{tabular}{|c|c|c|c|c|c|c|c|c|c|c|c|}
\hline \multirow{4}{*}{ INTERVALOS } & \multicolumn{11}{|c|}{ DER2 } \\
\hline & \multicolumn{4}{|c|}{ ZONA DE APNEA } & \multicolumn{7}{|c|}{ ZONA DE RESPIRACIÓN } \\
\hline & 0 & 5805 & 9235 & 12898 & 0 & 0 & 0 & 26706 & 0 & 0 & 0 \\
\hline & 0 & 7045 & 10475 & 14138 & 0 & 0 & 0 & 27946 & 0 & 0 & 0 \\
\hline \multicolumn{12}{|l|}{$\begin{array}{l}\text { BANDAS DE } \\
\text { FRECUENCIA }\end{array}$} \\
\hline $0-300$ & 0 & 294.68 & 359.68 & 540.12 & 0 & 0 & 0 & 372.18 & 0 & 0 & 0 \\
\hline $300-600$ & 0 & 74.072 & 91.755 & 74.148 & 0 & 0 & 0 & 73.746 & 0 & 0 & 0 \\
\hline $600-1200$ & 0 & 184.78 & 203.81 & 191.02 & 0 & 0 & 0 & 186.97 & 0 & 0 & 0 \\
\hline $\begin{array}{l}\text { BANDAS DE } \\
\text { FRECUENCIA }\end{array}$ & \multicolumn{11}{|c|}{ POTENCIAS DE LAS SEÑALES DE SONIDO RESPIRATORIO ESTIMADO SIN TW } \\
\hline $0-300$ & 0 & 244.22 & 203.92 & 442.09 & 0 & 0 & 0 & 332.07 & 0 & 0 & 0 \\
\hline $300-600$ & 0 & 6.7038 & 5.5596 & 6.1383 & 0 & 0 & 0 & 5.4303 & 0 & 0 & 0 \\
\hline $600-1200$ & 0 & 1.2433 & 2.6892 & 2.1892 & 0 & 0 & 0 & 2.2248 & 0 & 0 & 0 \\
\hline $\begin{array}{l}\text { BANDAS DE } \\
\text { FRECUENCIA }\end{array}$ & \multicolumn{11}{|c|}{ POTENCIAS DE LAS SEÑALES DE SONIDO RESPIRATORIO ESTIMADO CON TW } \\
\hline $0-300$ & 0 & 244.3 & 206.74 & 450.06 & 0 & 0 & 0 & 334.51 & 0 & 0 & 0 \\
\hline $300-600$ & 0 & 6.607 & 7.2669 & 10.583 & 0 & 0 & 0 & 10.651 & 0 & 0 & 0 \\
\hline $600-1200$ & 0 & 1.222 & 3.9755 & 3.8657 & 0 & 0 & 0 & 4.2674 & 0 & 0 & 0 \\
\hline $\begin{array}{l}\text { BANDAS DE } \\
\text { FRECUENCIA }\end{array}$ & \multicolumn{11}{|c|}{ POTENCIAS DE RUIDO CARDIACO ESTIMADO SIN TW } \\
\hline $0-300$ & 0 & 278.13 & 344.99 & 518.61 & 0 & 0 & 0 & 362.29 & 0 & 0 & 0 \\
\hline $300-600$ & 0 & 6.4496 & 5.6161 & 6.2088 & 0 & 0 & 0 & 5.7132 & 0 & 0 & 0 \\
\hline $600-1200$ & 0 & 1.2293 & 2.7006 & 2.2709 & 0 & 0 & 0 & 2.2351 & 0 & 0 & 0 \\
\hline $\begin{array}{l}\text { BANDAS DE } \\
\text { FRECUENCIA }\end{array}$ & \multicolumn{11}{|c|}{ POTENCIAS DE RUIDO CARDIACO ESTIMADO CON TW } \\
\hline $0-300$ & 0 & 277.92 & 353.48 & 518.49 & 0 & 0 & 0 & 362.08 & 0 & 0 & 0 \\
\hline $300-600$ & 0 & 6.422 & 6.5671 & 10.761 & 0 & 0 & 0 & 10.681 & 0 & 0 & 0 \\
\hline $600-1200$ & 0 & 1.2041 & 3.9701 & 3.9387 & 0 & 0 & 0 & 4.2452 & 0 & 0 & \\
\hline
\end{tabular}

Tabla 5. 3.e. Potencias calculadas de la señal en el dominio original para la línea axilar derecha media a un flujo de $0.5 \mathrm{~L} / \mathrm{seg}$.

\begin{tabular}{|c|c|c|c|c|c|c|c|c|c|c|c|}
\hline \multirow{4}{*}{ INTERVALOS } & \multicolumn{11}{|c|}{ DER3 } \\
\hline & \multicolumn{4}{|c|}{ ZONA DE APNEA } & \multicolumn{7}{|c|}{ ZONA DE RESPIRACIÓN } \\
\hline & 1804 & 5312 & 8956 & 12349 & 16565 & 20281 & 0 & 0 & 0 & 34940 & 0 \\
\hline & 3027 & 6535 & 10771 & 13949 & 18165 & 21881 & 0 & 0 & 0 & 36163 & 0 \\
\hline \multicolumn{12}{|l|}{$\begin{array}{l}\text { BANDAS DE } \\
\text { FRECUENCIA }\end{array}$} \\
\hline 0-300 & 1191.7 & 956.11 & 1687.5 & 1875.4 & 1329.2 & 1208.3 & 0 & 0 & 0 & 968.26 & 0 \\
\hline $300-600$ & 64.329 & 67.264 & 105.06 & 96.523 & 100 & 83.595 & 0 & 0 & 0 & 70.477 & 0 \\
\hline $600-1200$ & 159.23 & 150.54 & 234.62 & 215.58 & 202.65 & 194.48 & 0 & 0 & 0 & 181.31 & 0 \\
\hline $\begin{array}{l}\text { BANDAS DE } \\
\text { FRECUENCIA }\end{array}$ & \multicolumn{11}{|c|}{ POTENCIAS DE LAS SEÑALES DE SONIDO RESPIRATORIO ESTIMADO SIN TW } \\
\hline $0-300$ & 760.89 & 430.5 & 714.96 & 992.69 & 887.85 & 1137.6 & 0 & 0 & 0 & 800.95 & 0 \\
\hline $300-600$ & 18.022 & 6.4892 & 9.7712 & 26.256 & 10.381 & 18.657 & 0 & 0 & 0 & 13.911 & 0 \\
\hline $600-1200$ & 5.2037 & 2.2718 & 4.2339 & 10.846 & 4.2992 & 5.8171 & 0 & 0 & 0 & 5.7853 & 0 \\
\hline $\begin{array}{l}\text { BANDAS DE } \\
\text { FRECUENCIA }\end{array}$ & \multicolumn{11}{|c|}{ POTENCIAS DE LAS SEÑALES DE SONIDO RESPIRATORIO ESTIMADO CON TW } \\
\hline $0-300$ & 758.58 & 436.8 & 779.98 & 1058 & 908.27 & 1158.8 & 0 & 0 & 0 & 767.53 & 0 \\
\hline $300-600$ & 25.214 & 6.6624 & 45.599 & 45.987 & 7.914 & 45.581 & 0 & 0 & 0 & 24.494 & 0 \\
\hline $600-1200$ & 11.625 & 2.3326 & 25.573 & 25.662 & 4.1834 & 12.793 & 0 & 0 & 0 & 10.989 & 0 \\
\hline $\begin{array}{l}\text { BANDAS DE } \\
\text { FRECUENCIA }\end{array}$ & \multicolumn{11}{|c|}{ POTENCIAS DE RUIDO CARDIACO ESTIMADO SIN TW } \\
\hline $0-300$ & 1018 & 653.63 & 1251.1 & 1620.2 & 887.46 & 1011 & 0 & 0 & 0 & 704.34 & 0 \\
\hline $300-600$ & 17.89 & 6.4585 & 9.785 & 26.285 & 10.332 & 18.678 & 0 & 0 & 0 & 13.885 & 0 \\
\hline $600-1200$ & 5.2159 & 2.283 & 4.2538 & 10.842 & 4.2932 & 5.8176 & 0 & 0 & 0 & 5.7744 & 0 \\
\hline $\begin{array}{l}\text { BANDAS DE } \\
\text { FRECUENCIA }\end{array}$ & \multicolumn{11}{|c|}{ POTENCIAS DE RUIDO CARDIACO ESTIMADO CON TW } \\
\hline $0-300$ & 992.34 & 659.78 & 1293.8 & 1605 & 878.02 & 1025.2 & 0 & 0 & 0 & 738.14 & 0 \\
\hline $300-600$ & 25.253 & 6.6069 & 45.636 & 45.905 & 7.8722 & 45.634 & 0 & 0 & 0 & 24.497 & 0 \\
\hline $600-1200$ & 11.632 & 2.3466 & 25.582 & 25.679 & 4.1827 & 12.792 & 0 & 0 & 0 & 10.991 & 0 \\
\hline
\end{tabular}

Tabla 5. 3.f. Potencias calculadas de la señal en el dominio original para la línea axilar derecha basal a un flujo de $0.5 \mathrm{~L} / \mathrm{seg}$. 


\begin{tabular}{|c|c|c|c|c|c|c|c|c|c|c|c|}
\hline \multirow{4}{*}{ INTERVALOS } & \multicolumn{11}{|c|}{ MIT } \\
\hline & \multicolumn{4}{|c|}{ ZONA DE APNEA } & \multicolumn{7}{|c|}{ ZONA DE RESPIRACIÓN } \\
\hline & 1491 & 5151 & 9056 & 12785 & 16294 & 19840 & 23306 & 26907 & 30676 & 34772 & 38611 \\
\hline & 2618 & 6135 & 9916 & 13565 & 17305 & 20683 & 24149 & 27759 & 31832 & 35615 & 39391 \\
\hline \multicolumn{12}{|l|}{$\begin{array}{l}\text { BANDAS DE } \\
\text { FRECUENCIA }\end{array}$} \\
\hline 0-300 & 712.81 & 682.1 & 546.97 & 602.99 & 639.15 & 610.33 & 673.39 & 560.52 & 626.04 & 586.66 & 497.45 \\
\hline $300-600$ & 72.645 & 65.159 & 54.624 & 49.133 & 64.039 & 63.267 & 58.012 & 53.558 & 62.766 & 51.604 & 48.725 \\
\hline $600-1200$ & 175.07 & 170.14 & 117.89 & 133.44 & 162.14 & 147.21 & 141.79 & 150.24 & 183.94 & 139.71 & 124.32 \\
\hline $\begin{array}{l}\text { BANDAS DE } \\
\text { FRECUENCIA }\end{array}$ & \multicolumn{11}{|c|}{ POTENCIAS DE LAS SEÑALES DE SONIDO RESPIRATORIO ESTIMADO SIN TW } \\
\hline $0-300$ & 493.19 & 536.18 & 341.05 & 539.9 & 525.64 & 411.6 & 559.4 & 388.29 & 572.52 & 386.57 & 484.85 \\
\hline $300-600$ & 7.7753 & 10.35 & 14.136 & 4.9478 & 11.453 & 13.535 & 15.232 & 20.717 & 33.83 & 11.303 & 25.332 \\
\hline $600-1200$ & 3.6627 & 4.4521 & 4.4263 & 2.7861 & 7.3057 & 10.103 & 4.3939 & 6.9467 & 13.457 & 6.2867 & 10.88 \\
\hline $\begin{array}{l}\text { BANDAS DE } \\
\text { FRECUENCIA }\end{array}$ & \multicolumn{11}{|c|}{ POTENCIAS DE LAS SEÑALES DE SONIDO RESPIRATORIO ESTIMADO CON TW } \\
\hline $0-300$ & 507.31 & 563.13 & 392.8 & 559.91 & 563.17 & 539.26 & 505.1 & 440.99 & 636.54 & 360.9 & 540.9 \\
\hline $300-600$ & 13.047 & 28.033 & 62.535 & 36.72 & 39.959 & 189.07 & 20.211 & 198.42 & 228.68 & 13.362 & 118.79 \\
\hline $600-1200$ & 5.7554 & 18.885 & 30.136 & 44.523 & 32.178 & 100.68 & 10.671 & 101.55 & 123.12 & 11.835 & 81.352 \\
\hline $\begin{array}{l}\text { BANDAS DE } \\
\text { FRECUENCIA }\end{array}$ & \multicolumn{11}{|c|}{ POTENCIAS DE RUIDO CARDIACO ESTIMADO SIN TW } \\
\hline $0-300$ & 669.41 & 676.81 & 573.8 & 433.65 & 614.77 & 627.1 & 603.89 & 582.18 & 787.57 & 532.66 & 596.89 \\
\hline $300-600$ & 6.7429 & 10.382 & 13.813 & 3.8663 & 10.474 & 13.584 & & & & 10.955 & 25.246 \\
\hline $600-1200$ & 3.5359 & 4.4453 & 4.3735 & 2.6686 & 7.2254 & 10.093 & 4.3091 & 6.9269 & 13.444 & 6.229 & 10.878 \\
\hline $\begin{array}{l}\text { BANDAS DE } \\
\text { FRECUENCIA }\end{array}$ & \multicolumn{11}{|c|}{ POTENCIAS DE RUIDO CARDIACO ESTIMADO CON TW } \\
\hline $0-300$ & 647.67 & 667.66 & 596.25 & 447.92 & 642.66 & 734.69 & 572 & 628.78 & 943.91 & 491.59 & 669.83 \\
\hline $300-600$ & 11.994 & 27.892 & 62.613 & 36.481 & 39.908 & 188.86 & 18.902 & 198.42 & 228.7 & 13.311 & 118.76 \\
\hline $600-1200$ & 5.6517 & 18.853 & 30.183 & 44.411 & 32.081 & 100.68 & 10.578 & 101.55 & 123.11 & 11.809 & 81.338 \\
\hline
\end{tabular}

Tabla 5. 3.g. Potencias calculadas de la señal en el dominio original para la zona de auscultación de la válvula mitral a un flujo de $0.5 \mathrm{~L} / \mathrm{seg}$.

\begin{tabular}{|c|c|c|c|c|c|c|c|c|c|c|c|}
\hline \multirow{4}{*}{ INTERVALOS } & \multicolumn{11}{|c|}{ TRI } \\
\hline & \multicolumn{4}{|c|}{ ZONA DE APNEA } & \multicolumn{7}{|c|}{ ZONA DE RESPIRACIÓN } \\
\hline & 1325 & 5091 & 8723 & 12572 & 15953 & 19520 & 22958 & 26431 & 30392 & 34614 & 38670 \\
\hline & 2666 & 6432 & 10064 & 13346 & 17294 & 20861 & 24299 & 27772 & 31733 & 35388 & 39780 \\
\hline $\begin{array}{l}\text { BANDAS DE } \\
\text { FRECUENCIA }\end{array}$ & \multicolumn{11}{|c|}{ SEÑAL ORIGINAL } \\
\hline $0-300$ & 1163.1 & 1357.7 & 1302.1 & 1004 & 1637.3 & 1875.1 & 1441.6 & 1762.4 & 1815.8 & 1507.9 & 1685.9 \\
\hline $300-600$ & 77.024 & 62.677 & 84.892 & 51.431 & 81.69 & 71.361 & 75.611 & 92.615 & 83.384 & 48.174 & 66.695 \\
\hline $600-1200$ & 188.1 & 176.14 & 195.46 & 110.87 & 193.15 & 184.01 & 173.94 & 185.4 & 186.11 & 110.77 & 162.37 \\
\hline $\begin{array}{l}\text { BANDAS DE } \\
\text { FRECUENCIA }\end{array}$ & \multicolumn{11}{|c|}{ POTENCIAS DE LAS SEÑALES DE SONIDO RESPIRATORIO ESTIMADO SIN TW } \\
\hline $0-300$ & 609.43 & 669.74 & 769.69 & 358.79 & 854.87 & 774.61 & 807.98 & 748.48 & 1239.5 & 686.82 & 592.79 \\
\hline $300-600$ & 21.282 & 25.023 & 33.706 & 13.686 & 40.36 & 48.573 & 28.281 & 43.154 & 98.386 & 28.905 & 44.219 \\
\hline $600-1200$ & 7.2445 & 11.023 & 13.603 & 8.6955 & 17.113 & 24.126 & 11.6 & 19.669 & 57.141 & 12.432 & 24.876 \\
\hline $\begin{array}{l}\text { BANDAS DE } \\
\text { FRECUENCIA }\end{array}$ & \multicolumn{11}{|c|}{ POTENCIAS DE LAS SEÑALES DE SONIDO RESPIRATORIO ESTIMADO CON TW } \\
\hline $0-300$ & 579.76 & 630.52 & 762.71 & 387.71 & 834.89 & 975.53 & 792.77 & 957.43 & 1160.9 & 763.1 & 765.6 \\
\hline $300-600$ & 27.986 & 61.369 & 92.957 & 56.691 & 78.143 & 114.17 & 79.596 & 117.51 & 235.47 & 68.442 & 141.84 \\
\hline $600-1200$ & 13.794 & 23.105 & 43.529 & 22.491 & 38.541 & 56.835 & 40.421 & 46.65 & 116.59 & 40.964 & 66.998 \\
\hline $\begin{array}{l}\text { BANDAS DE } \\
\text { FRECUENCIA }\end{array}$ & \multicolumn{11}{|c|}{ POTENCIAS DE RUIDO CARDIACO ESTIMADO SIN TW } \\
\hline $0-300$ & 1096.7 & 1190.1 & 1080.9 & 921.82 & 1368.8 & 1913.2 & 1178 & 1723.2 & 1882.4 & 1418 & 1765.1 \\
\hline $300-600$ & 17.894 & 21.974 & 26.628 & 13.267 & 38.224 & 48.3 & 25.848 & 42.62 & 94.695 & 28.154 & 43.933 \\
\hline $600-1200$ & 6.8575 & 11 & 12.959 & 8.6667 & 16.918 & 23.97 & 11.44 & 19.453 & 56.923 & 12.412 & 24.817 \\
\hline $\begin{array}{l}\text { BANDAS DE } \\
\text { FRECUENCIA }\end{array}$ & \multicolumn{11}{|c|}{ POTENCIAS DE RUIDO CARDIACO ESTIMADO CON TW } \\
\hline $0-300$ & 1110.9 & 1228.8 & 1200.6 & 922.79 & 1411.4 & 1941.3 & 1210.9 & 1839.6 & 1843.3 & 1350.7 & 1762.1 \\
\hline $300-600$ & 24.429 & 58.762 & 89.357 & 57.23 & 76.482 & 113.09 & 79.702 & 115.51 & 232.22 & 68.888 & 140.98 \\
\hline $600-1200$ & 13.45 & 23.027 & 43.462 & 22.515 & 38.362 & 56.83 & 40.573 & 46.43 & 116.74 & 40.997 & 67.045 \\
\hline
\end{tabular}

Tabla 5. 3.h. Potencias calculadas de la señal en el dominio original para la zona de auscultación de la válvula tricúspide a un flujo de $0.5 \mathrm{~L} / \mathrm{seg}$. 


\begin{tabular}{|c|c|c|c|c|c|c|c|c|c|c|c|}
\hline \multirow{4}{*}{ INTERVALOS } & \multicolumn{11}{|c|}{ PUL } \\
\hline & \multicolumn{4}{|c|}{ ZONA DE APNEA } & \multicolumn{7}{|c|}{ ZONA DE RESPIRACIÓN } \\
\hline & 1589 & 5339 & 8935 & 12687 & 16427 & 19616 & 23220 & 26779 & 30589 & 34841 & 38800 \\
\hline & 2472 & 6257 & 9855 & 13790 & 17293 & 20523 & 24693 & 27645 & 31455 & 35801 & 39796 \\
\hline \multicolumn{12}{|l|}{$\begin{array}{l}\text { BANDAS DE } \\
\text { FRECUENCIA }\end{array}$} \\
\hline $0-300$ & 2865.2 & 2695.5 & 3024.9 & 3719.7 & 4446.5 & 3502.6 & 4388.8 & 3538.4 & 2268.3 & 3548.1 & 8759.2 \\
\hline $300-600$ & 81.444 & 75.312 & 104.95 & 158.2 & 82.344 & 92.445 & 103.73 & 85.627 & 60.751 & 66.787 & 79.915 \\
\hline $600-1200$ & 153.09 & 141.53 & 146.24 & 179.95 & 149.86 & 140.98 & 219.05 & 154.44 & 124.74 & 146.76 & 168.99 \\
\hline $\begin{array}{l}\text { BANDAS DE } \\
\text { FRECUENCIA }\end{array}$ & \multicolumn{11}{|c|}{ POTENCIAS DE LAS SEÑALES DE SONIDO RESPIRATORIO ESTIMADO SIN TW } \\
\hline $0-300$ & 2291.4 & 2410.2 & 2122.1 & 3009.5 & 2739.8 & 2120.3 & 3086.8 & 2148.2 & 1857.3 & 1771 & 3935.3 \\
\hline $300-600$ & 30.94 & 33.086 & 31.935 & 69.084 & 48.113 & 69.582 & 174.9 & 42.272 & 28.375 & 134.79 & 116.67 \\
\hline $600-1200$ & 6.604 & 4.9791 & 5.8318 & 20.648 & 9.4505 & 16.005 & 93.009 & 12.96 & 4.8864 & 81.09 & 80.617 \\
\hline $\begin{array}{l}\text { BANDAS DE } \\
\text { FRECUENCIA }\end{array}$ & \multicolumn{11}{|c|}{ POTENCIAS DE LAS SEÑALES DE SONIDO RESPIRATORIO ESTIMADO CON TW } \\
\hline $0-300$ & 2299.9 & 2409.5 & 2192.7 & 3007.8 & 2848.6 & 2152.5 & 3127.8 & 2271.7 & 1859.1 & 1658 & 4291.4 \\
\hline $300-600$ & 33.912 & 35.294 & 41.557 & 84.739 & 70.792 & 70.107 & 241.34 & 52.641 & 32.7 & 173.05 & 161.49 \\
\hline $600-1200$ & 7.8577 & 6.0349 & 11.201 & 28.471 & 19.31 & 24.291 & 118.42 & 17.893 & 8.5692 & 96.917 & 92.367 \\
\hline $\begin{array}{l}\text { BANDAS DE } \\
\text { FRECUENCIA }\end{array}$ & \multicolumn{11}{|c|}{ POTENCIAS DE RUIDO CARDIACO ESTIMADO SIN TW } \\
\hline $0-300$ & 2781.5 & 2616.4 & 2879.5 & 3367 & 2290.3 & 2881.4 & 3893.2 & 2176.8 & 2126 & 3057.5 & 5130 \\
\hline $300-600$ & 17.007 & 4.0884 & 11.219 & 41.643 & 19.549 & 51.987 & 165.91 & 26.641 & 11.379 & 131.03 & 105.03 \\
\hline $600-1200$ & 5.0731 & 1.6243 & 3.7402 & 18.375 & 6.3086 & 15.079 & 91.457 & 11.159 & 2.3841 & 80.461 & 80.402 \\
\hline $\begin{array}{l}\text { BANDAS DE } \\
\text { FRECUENCIA }\end{array}$ & \multicolumn{11}{|c|}{ POTENCIAS DE RUIDO CARDIACO ESTIMADO CON TW } \\
\hline $0-300$ & 2802.1 & 2619.7 & 2825.4 & 3349.6 & 2243.7 & 2808.6 & 4132 & 2051 & 2204.4 & 3119 & 5160.5 \\
\hline $300-600$ & 22.422 & 7.5937 & 26.199 & 58.989 & 45.598 & 53.87 & 236.02 & 41.08 & 18.506 & 169.73 & 154.8 \\
\hline $600-1200$ & 6.7425 & 2.9202 & 9.9083 & 27.011 & 16.882 & 24.374 & 117.76 & 17.098 & 7.0414 & 96.066 & 92.04 \\
\hline
\end{tabular}

Tabla 5. 3.i. Potencias calculadas de la señal en el dominio original obtenida de la zona de auscultación de la válvula pulmonar para un flujo de $0.5 \mathrm{~L} / \mathrm{seg}$.

\begin{tabular}{|c|c|c|c|c|c|c|c|c|c|c|c|}
\hline \multirow{4}{*}{ INTERVALOS } & \multicolumn{11}{|c|}{ AOR } \\
\hline & \multicolumn{4}{|c|}{ ZONA DE APNEA } & \multicolumn{7}{|c|}{ ZONA DE RESPIRACIÓN } \\
\hline & 1471 & 5039 & 8685 & 12592 & 16274 & 19390 & 22595 & 26543 & 30458 & 34290 & 38599 \\
\hline & 2717 & 6285 & 9950 & 13838 & 17182 & 20636 & 23982 & 27789 & 31704 & 35536 & 39507 \\
\hline $\begin{array}{l}\text { BANDAS DE } \\
\text { FRECUENCIA }\end{array}$ & \multicolumn{11}{|c|}{ SEÑAL ORIGINAL } \\
\hline $0-300$ & 1666.3 & 1175.2 & 1326 & 2611.7 & 1335.6 & 1609.7 & 1929.1 & 1600.7 & 1506.5 & 1674.1 & 1427.8 \\
\hline $300-600$ & 76.898 & 59.754 & 79.8 & 125.42 & 49.647 & 67.661 & 69.022 & 85.706 & 62.066 & 65.304 & 45.933 \\
\hline $600-1200$ & 174.82 & 144.13 & 157.74 & 165.58 & 117.02 & 170.52 & 168.61 & 165.64 & 159.22 & 167.33 & 126.74 \\
\hline $\begin{array}{l}\text { BANDAS DE } \\
\text { FRECUENCIA }\end{array}$ & \multicolumn{11}{|c|}{ POTENCIAS DE LAS SEÑALES DE SONIDO RESPIRATORIO ESTIMADO SIN TW } \\
\hline $0-300$ & 1299.8 & 748.06 & 999.36 & 1599 & 904.23 & 871.92 & 1184.2 & 1041.4 & 1247.3 & 1348.8 & 1190.6 \\
\hline $300-600$ & 14.893 & 20.216 & 22.602 & 17.383 & 20.306 & 26.682 & 19.081 & 24.006 & 20.432 & 28.454 & 26.983 \\
\hline $600-1200$ & 4.0266 & 3.3281 & 6.6923 & 7.0679 & 5.6739 & 14.617 & 7.0565 & 9.2554 & 6.9399 & 14.123 & 6.6199 \\
\hline $\begin{array}{l}\text { BANDAS DE } \\
\text { FRECUENCIA }\end{array}$ & \multicolumn{11}{|c|}{ POTENCIAS DE LAS SEÑALES DE SONIDO RESPIRATORIO ESTIMADO CON TW } \\
\hline $0-300$ & 1285 & 753.31 & 989.54 & 1633.4 & 928.39 & 860.24 & 1270.4 & 1043.1 & 1301.5 & 1348.2 & 1227.6 \\
\hline $300-600$ & 15.449 & 22.313 & 24.334 & 24.784 & 57.029 & 38.299 & 27.328 & 30.618 & 36.393 & 36.487 & 45.103 \\
\hline $600-1200$ & 7.4132 & 5.5343 & 8.2961 & 9.9642 & 17.808 & 21.379 & 11.668 & 12.841 & 12.142 & 20.745 & 19.902 \\
\hline $\begin{array}{l}\text { BANDAS DE } \\
\text { FRECUENCIA }\end{array}$ & \multicolumn{11}{|c|}{ POTENCIAS DE RUIDO CARDIACO ESTIMADO SIN TW } \\
\hline $0-300$ & 1523.6 & 1141.9 & 1302.7 & 1781.1 & 1172.9 & 1449.6 & 1551.2 & 1016.6 & 1203.7 & 1605.1 & 1233.1 \\
\hline $300-600$ & 12.672 & 17.788 & 21.502 & 15.611 & 19.347 & 23.325 & 15.73 & 20.064 & 19.222 & 27.814 & 26.276 \\
\hline $600-1200$ & 3.7449 & 3.3225 & 6.6055 & 6.917 & 5.6397 & 14.357 & 6.7581 & 8.8024 & 6.7534 & 13.975 & 6.5544 \\
\hline $\begin{array}{l}\text { BANDAS DE } \\
\text { FRECUENCIA }\end{array}$ & \multicolumn{11}{|c|}{ POTENCIAS DE RUIDO CARDIACO ESTIMADO CON TW } \\
\hline $0-300$ & 1488.3 & 1171.7 & 1281.5 & 1803.6 & 1243.3 & 1449.7 & 1508.4 & 1018.9 & 1204.8 & 1634.7 & 1248.4 \\
\hline $300-600$ & 12.285 & 20.611 & 23.002 & 23.918 & 57.075 & 35.464 & 24.185 & 29.965 & 35.449 & 35.443 & 44.833 \\
\hline $600-1200$ & 7.1882 & 5.5116 & 8.2377 & 9.8824 & 17.786 & 21.421 & 11.54 & 12.683 & 12.033 & 20.7 & 19.882 \\
\hline
\end{tabular}

Tabla 5. 3.j. Potencias calculadas de la señal en el dominio original obtenida de la zona de auscultación de la válvula aórtica para un flujo de $0.5 \mathrm{~L} / \mathrm{seg}$. 


\begin{tabular}{|c|c|c|c|c|c|c|c|c|c|c|c|}
\hline \multirow{4}{*}{ INTERVALOS } & \multicolumn{11}{|c|}{ PRE } \\
\hline & \multicolumn{4}{|c|}{ ZONA DE APNEA } & \multicolumn{7}{|c|}{ ZONA DE RESPIRACIÓN } \\
\hline & 1406 & 5111 & 8764 & 12527 & 16066 & 19659 & 23079 & 26767 & 30489 & 34606 & 38601 \\
\hline & 2482 & 6187 & 9901 & 13603 & 17340 & 20735 & 24155 & 27910 & 31596 & 35749 & 39677 \\
\hline $\begin{array}{l}\text { BANDAS DE } \\
\text { FRECUENCIA }\end{array}$ & \multicolumn{11}{|c|}{ SEÑAL ORIGINAL } \\
\hline $0-300$ & 737.73 & 816.56 & 859.88 & 936.98 & 871.72 & 838.26 & 863.68 & 940.6 & 849.94 & 866.2 & 804.55 \\
\hline $300-600$ & 64.874 & 56.444 & 71.337 & 64.783 & 65.69 & 62.365 & 60.644 & 77.394 & 55.507 & 70.716 & 61.694 \\
\hline $600-1200$ & 180.59 & 158.85 & 171.95 & 163.39 & 184.11 & 160.37 & 178.5 & 168.85 & 159.64 & 186.54 & 148.71 \\
\hline $\begin{array}{l}\text { BANDAS DE } \\
\text { FRECUENCIA }\end{array}$ & \multicolumn{11}{|c|}{ POTENCIAS DE LAS SEÑALES DE SONIDO RESPIRATORIO ESTIMADO SIN TW } \\
\hline $0-300$ & 345.8 & 457.4 & 353.64 & 515.75 & 769.35 & 414.03 & 480.29 & 628.4 & 398.48 & 624.18 & 489.32 \\
\hline $300-600$ & 7.7504 & 6.0357 & 5.9764 & 8.2195 & 56.218 & 24.992 & 22.414 & 38.424 & 21.724 & 28.239 & 14.974 \\
\hline $600-1200$ & 1.6155 & 2.5432 & 3.7173 & 4.0411 & 40.988 & 13.059 & 13.259 & 15.728 & 17.97 & 14.993 & 7.5267 \\
\hline $\begin{array}{l}\text { BANDAS DE } \\
\text { FRECUENCIA }\end{array}$ & \multicolumn{11}{|c|}{ POTENCIAS DE LAS SEÑALES DE SONIDO RESPIRATORIO ESTIMADO CON TW } \\
\hline $0-300$ & 401.92 & 430.13 & 353.52 & 505.21 & 978.37 & 452.73 & 533.53 & 730.4 & 386.01 & 673.28 & 424.7 \\
\hline $300-600$ & 12.217 & 23.608 & 5.9252 & 25.889 & 306.51 & 156.23 & 125.72 & 173.24 & 62.298 & 95.67 & 29.529 \\
\hline $600-1200$ & 3.6137 & 9.4481 & 3.913 & 9.749 & 161.99 & 109.04 & 84.309 & 65.524 & 71.396 & 50.298 & 28.036 \\
\hline $\begin{array}{l}\text { BANDAS DE } \\
\text { FRECUENCIA }\end{array}$ & \multicolumn{11}{|c|}{ POTENCIAS DE RUIDO CARDIACO ESTIMADO SIN TW } \\
\hline $0-300$ & 749.59 & 707.34 & 832.02 & 661.02 & 968.68 & 904.64 & 854.43 & 930.04 & 885.59 & 907.8 & 792.65 \\
\hline $300-600$ & 7.7683 & 5.9845 & 6.0598 & 8.0927 & 56.23 & 24.926 & 22.256 & 38.236 & 21.647 & 28.212 & 15.094 \\
\hline $600-1200$ & 1.6105 & 2.5289 & 3.7039 & 4.0442 & 40.985 & 13.058 & 13.244 & 15.723 & 17.981 & 14.993 & 7.524 \\
\hline $\begin{array}{l}\text { BANDAS DE } \\
\text { FRECUENCIA }\end{array}$ & \multicolumn{11}{|c|}{ POTENCIAS DE RUIDO CARDIACO ESTIMADO CON TW } \\
\hline $0-300$ & 746.21 & 744.95 & 831.95 & 674.38 & 1200.9 & 894.32 & 846.25 & 1029 & 844.42 & 856.14 & 792.06 \\
\hline $300-600$ & 12.126 & 23.647 & 5.8959 & 25.796 & 306.54 & 156.37 & 125.6 & 173.06 & 62.287 & 95.561 & 29.451 \\
\hline $600-1200$ & 3.6038 & 9.4548 & 3.8976 & 9.7568 & 161.99 & 109.04 & 84.321 & 65.525 & 71.394 & 50.298 & 28.038 \\
\hline
\end{tabular}

Tabla 5. 3.k. Potencias calculadas de la señal en el dominio original obtenida de la zona de auscultación precordial promedio para un flujo de $0.5 \mathrm{~L} / \mathrm{seg}$ 
Tabla 5. 4 Tablas de Potencias calculadas de la señal en el dominio original obtenida en los puntos adquiridos para flujo de $1.0 \mathrm{~L} / \mathrm{seg}$

\begin{tabular}{|c|c|c|c|c|c|c|c|c|c|c|c|}
\hline \multirow{4}{*}{ INTERVALOS } & \multicolumn{11}{|c|}{ CANAL IZQ1 } \\
\hline & \multicolumn{4}{|c|}{ ZONA DE APNEA } & \multicolumn{7}{|c|}{ ZONA DE RESPIRACIÓN } \\
\hline & 0 & 3049 & 6542 & 10705 & 14278 & 18047 & 21787 & 26075 & 0 & 0 & 38915 \\
\hline & 0 & 4877 & 8370 & 11913 & 15486 & 19278 & 23615 & 27903 & 0 & 0 & 40743 \\
\hline $\begin{array}{l}\text { BANDAS DE } \\
\text { FRECUENCIA }\end{array}$ & \multicolumn{11}{|c|}{ SEÑAL ORIGINAL } \\
\hline $0-300$ & 0 & 658.09 & 645.3 & 684.9 & 836.25 & 847.93 & 894.61 & 1147 & 0 & 0 & 1071 \\
\hline $300-600$ & 0 & 90.212 & 102.53 & 74.923 & 77.614 & 76.04 & 126.01 & 114.65 & 0 & 0 & 118.89 \\
\hline $600-1200$ & 0 & 252.48 & 255.54 & 191.74 & 188.02 & 189.57 & 270.97 & 252.41 & 0 & 0 & 265.15 \\
\hline $\begin{array}{l}\text { BANDAS DE } \\
\text { FRECUENCIA }\end{array}$ & \multicolumn{11}{|c|}{ POTENCIAS DE LAS SEÑALES DE SONIDO RESPIRATORIO ESTIMADO SIN TW } \\
\hline $0-300$ & 0 & 497.73 & 519.79 & 513.33 & 741.74 & 1086.8 & 711.48 & 1100.5 & 0 & 0 & 971.69 \\
\hline $300-600$ & 0 & 16.118 & 12.874 & 16.414 & 19.224 & 17.009 & 25.802 & 27.203 & 0 & 0 & 31.165 \\
\hline $600-1200$ & 0 & 4.9096 & 3.1714 & 4.8959 & 10.47 & 6.5522 & 8.8412 & 13.421 & 0 & 0 & 12.897 \\
\hline $\begin{array}{l}\text { BANDAS DE } \\
\text { FRECUENCIA }\end{array}$ & \multicolumn{11}{|c|}{ POTENCIAS DE LAS SEÑALES DE SONIDO RESPIRATORIO ESTIMADO CON TW } \\
\hline $0-300$ & 0 & 533.26 & 512.07 & 493.85 & 725.77 & 1088.1 & 716.16 & 1082 & 0 & 0 & 1064 \\
\hline $300-600$ & 0 & 34.759 & 15.636 & 23.147 & 31.398 & 26.862 & 33.997 & 38.38 & 0 & 0 & 68.479 \\
\hline $600-1200$ & 0 & 9.0521 & 3.8708 & 10.281 & 21.09 & 13.865 & 15.203 & 22.195 & 0 & 0 & 26.048 \\
\hline $\begin{array}{l}\text { BANDAS DE } \\
\text { FRECUENCIA }\end{array}$ & \multicolumn{11}{|c|}{ POTENCIAS DE RUIDO CARDIACO ESTIMADO SIN TW } \\
\hline $0-300$ & 0 & 614.76 & 560.21 & 585.84 & 587.26 & 740.65 & 747.91 & 881.07 & 0 & 0 & 907.8 \\
\hline $300-600$ & 0 & 12.66 & 9.1057 & 14.596 & 17.65 & 14.748 & 23.988 & 24.256 & 0 & 0 & 30.858 \\
\hline $600-1200$ & 0 & 4.5907 & 2.7516 & 4.5654 & 10.297 & 6.1815 & 8.5402 & 13.176 & 0 & 0 & 12.482 \\
\hline $\begin{array}{l}\text { BANDAS DE } \\
\text { FRECUENCIA }\end{array}$ & \multicolumn{11}{|c|}{ POTENCIAS DE RUIDO CARDIACO ESTIMADO CON TW } \\
\hline $0-300$ & 0 & 612.68 & 557.68 & 613.01 & 630.46 & 747.76 & 753.14 & 937.21 & 0 & 0 & 889.17 \\
\hline $300-600$ & 0 & 31.486 & 11.759 & 21.786 & 29.217 & 23.435 & 31.492 & 37.289 & 0 & 0 & 65.855 \\
\hline $600-1200$ & 0 & 9.0263 & 3.5169 & 10.037 & 20.964 & 13.877 & 14.974 & 21.895 & 0 & 0 & 25.899 \\
\hline
\end{tabular}

Tabla 5. 4.a. Potencias calculadas de la señal en el dominio original obtenida de la línea axilar izquierda apical para un flujo de $1.0 \mathrm{~L} / \mathrm{seg}$.

\begin{tabular}{|c|c|c|c|c|c|c|c|c|c|c|c|}
\hline \multirow{4}{*}{ INTERVALOS } & \multicolumn{11}{|c|}{ CANAL IZQ2 } \\
\hline & \multicolumn{4}{|c|}{ ZONA DE APNEA } & \multicolumn{7}{|c|}{ ZONA DE RESPIRACIÓN } \\
\hline & 209 & 3638 & 7200 & 10870 & 14156 & 17857 & 0 & 26393 & 30878 & 34838 & 39130 \\
\hline & 1673 & 4923 & 8485 & 12155 & 15441 & 19142 & 0 & 27810 & 32163 & 36123 & 40415 \\
\hline $\begin{array}{l}\text { BANDAS DE } \\
\text { FRECUENCIA }\end{array}$ & \multicolumn{11}{|c|}{ SEÑAL ORIGINAL } \\
\hline $0-300$ & 1950.2 & 1460.7 & 1955.2 & 2020.6 & 2771.4 & 2980.3 & 0 & 3554.3 & 4772.9 & 1826.2 & 2747.5 \\
\hline $300-600$ & 79.789 & 63.977 & 76.086 & 68.319 & 69.119 & 71.694 & 0 & 81.907 & 74.029 & 92.697 & 79.84 \\
\hline $600-1200$ & 187.58 & 162.23 & 168.34 & 155.91 & 178 & 167.03 & 0 & 186.95 & 186.35 & 171.14 & 176.8 \\
\hline $\begin{array}{l}\text { BANDAS DE } \\
\text { FRECUENCIA }\end{array}$ & \multicolumn{11}{|c|}{ POTENCIAS DE LAS SEÑALES DE SONIDO RESPIRATORIO ESTIMADO SIN TW } \\
\hline $0-300$ & 1138 & 729 & 1214.2 & 1339.5 & 2107.7 & 2126.3 & 0 & 2623.9 & 3009 & 971.35 & 1863.8 \\
\hline $300-600$ & 47.763 & 29.914 & 54.457 & 68.404 & 72.054 & 71.383 & 0 & 120.97 & 145.14 & 47.995 & 49.613 \\
\hline $600-1200$ & 14.855 & 8.5006 & 17.409 & 17.386 & 32.364 & 35.161 & 0 & 64.881 & 98.406 & 11.934 & 9.8811 \\
\hline $\begin{array}{l}\text { BANDAS DE } \\
\text { FRECUENCIA }\end{array}$ & \multicolumn{11}{|c|}{ POTENCIAS DE LAS SEÑALES DE SONIDO RESPIRATORIO ESTIMADO CON TW } \\
\hline $0-300$ & 1144.3 & 724.86 & 1140.4 & 1363.8 & 2061.5 & 2332.7 & 0 & 2813.8 & 3302.5 & 919.64 & 1978 \\
\hline $300-600$ & 77.201 & 29.473 & 71.942 & 122.31 & 104.16 & 104.5 & 0 & 243.93 & 275.29 & 88.81 & 71.21 \\
\hline $600-1200$ & 22.39 & 7.9347 & 25.792 & 38.017 & 61.014 & 61.229 & 0 & 106.43 & 168.76 & 20.578 & 16.55 \\
\hline $\begin{array}{l}\text { BANDAS DE } \\
\text { FRECUENCIA }\end{array}$ & \multicolumn{11}{|c|}{ POTENCIAS DE RUIDO CARDIACO ESTIMADO SIN TW } \\
\hline $0-300$ & 1755.6 & 946.6 & 1672.3 & 2049.8 & 1589.7 & 2150.2 & 0 & 3468.3 & 4572.9 & 1324.6 & 1375.5 \\
\hline $300-600$ & 28.694 & 3.996 & 38.496 & 53.131 & 58.806 & 56.531 & 0 & 116.23 & 137.52 & 25.608 & 20.852 \\
\hline $600-1200$ & 11.448 & 4.6299 & 14.622 & 15.839 & 30.761 & 32.709 & 0 & 63.08 & 97.393 & 9.5741 & 6.6589 \\
\hline $\begin{array}{l}\text { BANDAS DE } \\
\text { FRECUENCIA }\end{array}$ & \multicolumn{11}{|c|}{ POTENCIAS DE RUIDO CARDIACO ESTIMADO CON TW } \\
\hline $0-300$ & 1768.1 & 943.49 & 1702 & 2015.5 & 1522.3 & 2180.5 & 0 & 3466.8 & 4811.9 & 1291.5 & 1428.5 \\
\hline $300-600$ & 62.562 & 3.2931 & 65.228 & 111.07 & 95.389 & 98.551 & 0 & 237.09 & 273.73 & 73.745 & 46.282 \\
\hline $600-1200$ & 19.484 & 4.0726 & 23.514 & 36.922 & 60.344 & 60.176 & 0 & 105.76 & 168.89 & 18.993 & 15.577 \\
\hline
\end{tabular}

Tabla 5. 4.b. Potencias calculadas de la señal en el dominio original obtenida de la línea axilar izquierda media para un flujo de $1.0 \mathrm{~L} / \mathrm{seg}$. 


\begin{tabular}{|c|c|c|c|c|c|c|c|c|c|c|c|}
\hline \multirow{4}{*}{ INTERVALOS } & \multicolumn{11}{|c|}{ CANAL IZQ3 } \\
\hline & \multicolumn{4}{|c|}{ ZONA DE APNEA } & \multicolumn{7}{|c|}{ ZONA DE RESPIRACIÓN } \\
\hline & 68 & 3739 & 7082 & 9651 & 14409 & 18232 & 22295 & 26819 & 30478 & 0 & 39175 \\
\hline & 1101 & 4627 & 8115 & 11647 & 15631 & 19316 & 23387 & 27852 & 31511 & 0 & 40208 \\
\hline $\begin{array}{l}\text { BANDAS DE } \\
\text { FRECUENCIA }\end{array}$ & \multicolumn{11}{|c|}{ SEÑAL ORIGINAL } \\
\hline $0-300$ & 683.17 & 474.06 & 685.49 & 1346.2 & 981.04 & 977.18 & 920.7 & 1030.2 & 817.82 & 0 & 941.13 \\
\hline $300-600$ & 60.56 & 55.249 & 65.007 & 132.57 & 87.418 & 63.048 & 82.609 & 62.153 & 61.41 & 0 & 72.629 \\
\hline $600-1200$ & 166.95 & 144.36 & 163.4 & 321.61 & 200.55 & 153.38 & 194.62 & 150.78 & 155.5 & 0 & 160.6 \\
\hline $\begin{array}{l}\text { BANDAS DE } \\
\text { FRECUENCIA }\end{array}$ & \multicolumn{11}{|c|}{ POTENCIAS DE LAS SEÑALES DE SONIDO RESPIRATORIO ESTIMADO SIN TW } \\
\hline $0-300$ & 588.42 & 328.75 & 375.67 & 827.34 & 439.32 & 675.55 & 453.37 & 564.41 & 693.15 & 0 & 564.8 \\
\hline $300-600$ & 17.115 & 8.7559 & 8.4891 & 22.968 & 17.51 & 30.479 & 24.729 & 14.028 & 22.659 & 0 & 14.649 \\
\hline $600-1200$ & 2.651 & 1.0535 & 1.3348 & 4.3016 & 4.0093 & 14.999 & 14.003 & 6.9027 & 10.901 & 0 & 6.7559 \\
\hline $\begin{array}{l}\text { BANDAS DE } \\
\text { FRECUENCIA }\end{array}$ & \multicolumn{11}{|c|}{ POTENCIAS DE LAS SEÑALES DE SONIDO RESPIRATORIO ESTIMADO CON TW } \\
\hline $0-300$ & 555.22 & 328.6 & 375.46 & 839.44 & 415.93 & 645.76 & 437.02 & 595.78 & 718.72 & 0 & 567.94 \\
\hline $300-600$ & 14.315 & 8.7493 & 8.4968 & 23.267 & 23.316 & 54.112 & 29.566 & 19.196 & 30.133 & 0 & 20.969 \\
\hline $600-1200$ & 2.9988 & 1.0836 & 1.325 & 5.7597 & 5.7213 & 24.742 & 18 & 9.0016 & 15.705 & 0 & 11.542 \\
\hline $\begin{array}{l}\text { BANDAS DE } \\
\text { FRECUENCIA }\end{array}$ & \multicolumn{11}{|c|}{ POTENCIAS DE RUIDO CARDIACO ESTIMADO SIN TW } \\
\hline $0-300$ & 343.17 & 176.52 & 331.77 & 680.93 & 597.07 & 721.2 & 665.15 & 696.95 & 559.02 & 0 & 624.56 \\
\hline $300-600$ & 9.1947 & 0.52542 & 1.2649 & 13.721 & 11.165 & 28.075 & 19.492 & 11.487 & 20.004 & 0 & 12.4 \\
\hline $600-1200$ & 2.2178 & 0.18638 & 0.67866 & 3.1529 & 3.5181 & 15.011 & 13.537 & 6.4993 & 10.54 & 0 & 6.4488 \\
\hline $\begin{array}{l}\text { BANDAS DE } \\
\text { FRECUENCIA }\end{array}$ & \multicolumn{11}{|c|}{ POTENCIAS DE RUIDO CARDIACO ESTIMADO CON TW } \\
\hline 0-300 & 334.58 & 176.59 & 331.46 & 704.46 & 585.9 & 707.77 & 667.41 & 695.66 & 548.69 & 0 & 635.84 \\
\hline $300-600$ & 7.1143 & 0.55089 & 1.2161 & 12.182 & 17.754 & 52.756 & 25.906 & 18.182 & 27.853 & 0 & 19.615 \\
\hline $600-1200$ & 2.5309 & 0.23183 & 0.67537 & 4.8478 & 5.3872 & 24.731 & 17.721 & 8.8463 & 15.444 & 0 & 11.275 \\
\hline
\end{tabular}

Tabla 5. 4.c. Potencias calculadas de la señal en el dominio original obtenida de la línea axilar izquierda basal para un flujo de $1.0 \mathrm{~L} / \mathrm{seg}$.

\begin{tabular}{|c|c|c|c|c|c|c|c|c|c|c|c|}
\hline \multirow{4}{*}{ INTERVALOS } & \multicolumn{11}{|c|}{ DER1 } \\
\hline & \multicolumn{4}{|c|}{ ZONA DE APNEA } & \multicolumn{7}{|c|}{ ZONA DE RESPIRACIÓN } \\
\hline & 0 & 3630 & 7132 & 10726 & 14096 & 18007 & 21886 & 26698 & 30861 & 34973 & 38896 \\
\hline & 0 & 4762 & 7853 & 11447 & 15228 & 19139 & 23018 & 27845 & 31993 & 35694 & 40028 \\
\hline $\begin{array}{l}\text { BANDAS DE } \\
\text { FRECUENCIA }\end{array}$ & \multicolumn{11}{|c|}{ SEÑAL ORIGINAL } \\
\hline $0-300$ & 0 & 389.14 & 296.44 & 272.31 & 754.67 & 526.94 & 814.38 & 575.93 & 615.5 & 287.01 & 557.03 \\
\hline $300-600$ & 0 & 47.464 & 33.828 & 40.549 & 87.866 & 66.782 & 83.903 & 59.657 & 63.179 & 35.422 & 62.776 \\
\hline $600-1200$ & 0 & 144.6 & 93.855 & 94.486 & 160.18 & 149.43 & 157.31 & 152.95 & 142.85 & 93.612 & 147.56 \\
\hline $\begin{array}{l}\text { BANDAS DE } \\
\text { FRECUENCIA }\end{array}$ & \multicolumn{11}{|c|}{ POTENCIAS DE LAS SEÑALES DE SONIDO RESPIRATORIO ESTIMADO SIN TW } \\
\hline $0-300$ & 0 & 261.82 & 168.14 & 149.78 & 488.07 & 402.74 & 561.78 & 439.1 & 422.83 & 228.16 & 348.51 \\
\hline $300-600$ & 0 & 4.5249 & 2.9461 & 2.1708 & 6.7597 & 6.4177 & 15.047 & 16.35 & 4.7539 & 7.1427 & 6.7449 \\
\hline $600-1200$ & 0 & 1.1845 & 0.27693 & 1.1572 & 2.3202 & 1.8401 & 4.6271 & 6.0535 & 1.7153 & 1.6009 & 2.1253 \\
\hline $\begin{array}{l}\text { BANDAS DE } \\
\text { FRECUENCIA }\end{array}$ & \multicolumn{11}{|c|}{ POTENCIAS DE LAS SEÑALES DE SONIDO RESPIRATORIO ESTIMADO CON TW } \\
\hline $0-300$ & 0 & 264.91 & 171.74 & 166.11 & 483.04 & 404.48 & 602.27 & 390.41 & 427.96 & 292 & 336.86 \\
\hline $300-600$ & 0 & 4.5038 & 4.3422 & 21.399 & 14.223 & 12.349 & 25.249 & 25.981 & 5.84 & 30.351 & 18.62 \\
\hline $600-1200$ & 0 & 2.5197 & 0.40614 & 17.726 & 12.242 & 4.8715 & 16.114 & 24.926 & 2.9217 & 20.143 & 10.754 \\
\hline $\begin{array}{c}\text { BANDAS DE } \\
\text { FRECUENCIA }\end{array}$ & \multicolumn{11}{|c|}{ POTENCIAS DE RUIDO CARDIACO ESTIMADO SIN TW } \\
\hline $0-300$ & 0 & 211.71 & 152.66 & 138.11 & 279.31 & 218.72 & 387.54 & 479.4 & 246.01 & 196.69 & 267.34 \\
\hline $300-600$ & 0 & 4.1611 & 3.1005 & 2.1622 & 6.3871 & 6.2722 & 13.899 & 16.431 & 4.4796 & 7.1509 & 6.8168 \\
\hline $600-1200$ & 0 & 1.1502 & 0.27688 & 1.1514 & 2.2939 & 1.8432 & 4.68 & 6.0391 & 1.6873 & 1.6041 & 2.1113 \\
\hline $\begin{array}{l}\text { BANDAS DE } \\
\text { FRECUENCIA }\end{array}$ & \multicolumn{11}{|c|}{ POTENCIAS DE RUIDO CARDIACO ESTIMADO CON TW } \\
\hline $0-300$ & 0 & 211 & 156.16 & 139.56 & 289.6 & 209.53 & 356.92 & 452.07 & 251.64 & 218.53 & 275.39 \\
\hline $300-600$ & 0 & 4.222 & 4.7368 & 21.406 & 13.931 & 12.256 & 26.13 & 25.898 & 5.6583 & 30.502 & 18.756 \\
\hline $600-1200$ & 0 & 2.4973 & 0.43173 & 17.728 & 12.193 & 4.8748 & 16.113 & 24.917 & 2.9213 & 20.146 & 10.76 \\
\hline
\end{tabular}

Tabla 5. 4.d. Potencias calculadas de la señal en el dominio original obtenida de la línea axilar derecha apical para un flujo de 1.0L/seg. 


\begin{tabular}{|c|c|c|c|c|c|c|c|c|c|c|c|}
\hline \multirow{4}{*}{ INTERVALOS } & \multicolumn{11}{|c|}{ DER2 } \\
\hline & \multicolumn{4}{|c|}{ ZONA DE APNEA } & \multicolumn{7}{|c|}{ ZONA DE RESPIRACIÓN } \\
\hline & 0 & 0 & 0 & 0 & 0 & 0 & 0 & 0 & 0 & 0 & 0 \\
\hline & 0 & 0 & 0 & 0 & 0 & 0 & 0 & 0 & 0 & 0 & 0 \\
\hline \multicolumn{12}{|l|}{$\begin{array}{l}\text { BANDAS DE } \\
\text { FRECUENCIA }\end{array}$} \\
\hline $0-300$ & 0 & 0 & 0 & 0 & 0 & 0 & 0 & 0 & 0 & 0 & 0 \\
\hline $300-600$ & 0 & 0 & 0 & 0 & 0 & 0 & 0 & 0 & 0 & 0 & 0 \\
\hline $600-1200$ & 0 & 0 & 0 & 0 & 0 & 0 & 0 & 0 & 0 & 0 & 0 \\
\hline $\begin{array}{l}\text { BANDAS DE } \\
\text { FRECUENCIA }\end{array}$ & \multicolumn{11}{|c|}{ POTENCIAS DE LAS SEÑALES DE SONIDO RESPIRATORIO ESTIMADO SIN TW } \\
\hline $0-300$ & 0 & 0 & 0 & 0 & 0 & 0 & 0 & 0 & 0 & 0 & 0 \\
\hline $300-600$ & 0 & 0 & 0 & 0 & 0 & 0 & 0 & 0 & 0 & 0 & 0 \\
\hline $600-1200$ & 0 & 0 & 0 & 0 & 0 & 0 & 0 & 0 & 0 & 0 & 0 \\
\hline $\begin{array}{l}\text { BANDAS DE } \\
\text { FRECUENCIA }\end{array}$ & \multicolumn{11}{|c|}{ POTENCIAS DE LAS SEÑALES DE SONIDO RESPIRATORIO ESTIMADO CON TW } \\
\hline $0-300$ & 0 & 0 & 0 & 0 & 0 & 0 & 0 & 0 & 0 & 0 & 0 \\
\hline $300-600$ & 0 & 0 & 0 & 0 & 0 & 0 & 0 & 0 & 0 & 0 & 0 \\
\hline 600-1200 & 0 & 0 & 0 & 0 & 0 & 0 & 0 & 0 & 0 & 0 & 0 \\
\hline $\begin{array}{l}\text { BANDAS DE } \\
\text { FRECUENCIA }\end{array}$ & \multicolumn{11}{|c|}{ POTENCIAS DE RUIDO CARDIACO ESTIMADO SIN TW } \\
\hline $0-300$ & 0 & 0 & 0 & 0 & 0 & 0 & 0 & 0 & 0 & 0 & 0 \\
\hline $300-600$ & 0 & 0 & 0 & 0 & 0 & 0 & 0 & 0 & 0 & 0 & 0 \\
\hline $600-1200$ & 0 & 0 & 0 & 0 & 0 & 0 & 0 & 0 & 0 & 0 & 0 \\
\hline $\begin{array}{l}\text { BANDAS DE } \\
\text { FRECUENCIA }\end{array}$ & \multicolumn{11}{|c|}{ POTENCIAS DE RUIDO CARDIACO ESTIMADO CON TW } \\
\hline $0-300$ & 0 & 0 & 0 & 0 & 0 & 0 & 0 & 0 & 0 & 0 & 0 \\
\hline $300-600$ & 0 & 0 & 0 & 0 & 0 & 0 & 0 & 0 & 0 & 0 & 0 \\
\hline $600-1200$ & 0 & 0 & 0 & 0 & 0 & 0 & 0 & 0 & 0 & 0 & 0 \\
\hline
\end{tabular}

Tabla 5. 4.e. Potencias calculadas de la señal en el dominio original obtenida de la línea axilar derecha media para un flujo de $1.0 \mathrm{~L} / \mathrm{seg}$.

\begin{tabular}{|c|c|c|c|c|c|c|c|c|c|c|c|}
\hline \multirow{4}{*}{ INTERVALOS } & \multicolumn{11}{|c|}{ DER3 } \\
\hline & \multicolumn{4}{|c|}{ ZONA DE APNEA } & \multicolumn{7}{|c|}{ ZONA DE RESPIRACIÓN } \\
\hline & 0 & 0 & 0 & 0 & 0 & 0 & 0 & 0 & 0 & 0 & 0 \\
\hline & 0 & 0 & 0 & 0 & 0 & 0 & 0 & 0 & 0 & 0 & 0 \\
\hline $\begin{array}{l}\text { BANDAS DE } \\
\text { FRECUENCIA }\end{array}$ & \multicolumn{11}{|c|}{ SEÑAL ORIGINAL } \\
\hline $0-300$ & 0 & 0 & 0 & 0 & 0 & 0 & 0 & 0 & 0 & 0 & 0 \\
\hline $300-600$ & 0 & 0 & 0 & 0 & 0 & 0 & 0 & 0 & 0 & 0 & 0 \\
\hline $600-1200$ & 0 & 0 & 0 & 0 & 0 & 0 & 0 & 0 & 0 & 0 & 0 \\
\hline $\begin{array}{l}\text { BANDAS DE } \\
\text { FRECUENCIA }\end{array}$ & \multicolumn{11}{|c|}{ POTENCIAS DE LAS SEÑALES DE SONIDO RESPIRATORIO ESTIMADO SIN TW } \\
\hline $0-300$ & 0 & 0 & 0 & 0 & 0 & 0 & 0 & 0 & 0 & 0 & 0 \\
\hline $300-600$ & 0 & 0 & 0 & 0 & 0 & 0 & 0 & 0 & 0 & 0 & 0 \\
\hline $600-1200$ & 0 & 0 & 0 & 0 & 0 & 0 & 0 & 0 & 0 & 0 & 0 \\
\hline $\begin{array}{l}\text { BANDAS DE } \\
\text { FRECUENCIA }\end{array}$ & \multicolumn{11}{|c|}{ POTENCIAS DE LAS SEÑALES DE SONIDO RESPIRATORIO ESTIMADO CON TW } \\
\hline $0-300$ & 0 & 0 & 0 & 0 & 0 & 0 & 0 & 0 & 0 & 0 & 0 \\
\hline $300-600$ & 0 & 0 & 0 & 0 & 0 & 0 & 0 & 0 & 0 & 0 & 0 \\
\hline $600-1200$ & 0 & 0 & 0 & 0 & 0 & 0 & 0 & 0 & 0 & 0 & 0 \\
\hline $\begin{array}{l}\text { BANDAS DE } \\
\text { FRECUENCIA }\end{array}$ & \multicolumn{11}{|c|}{ POTENCIAS DE RUIDO CARDIACO ESTIMADO SIN TW } \\
\hline $0-300$ & 0 & 0 & 0 & 0 & 0 & 0 & 0 & 0 & 0 & 0 & 0 \\
\hline $300-600$ & 0 & 0 & 0 & 0 & 0 & 0 & 0 & 0 & 0 & 0 & 0 \\
\hline $600-1200$ & 0 & 0 & 0 & 0 & 0 & 0 & 0 & 0 & 0 & 0 & 0 \\
\hline $\begin{array}{l}\text { BANDAS DE } \\
\text { FRECUENCIA }\end{array}$ & \multicolumn{11}{|c|}{ POTENCIAS DE RUIDO CARDIACO ESTIMADO CON TW } \\
\hline $0-300$ & 0 & 0 & 0 & 0 & 0 & 0 & 0 & 0 & 0 & 0 & 0 \\
\hline $300-600$ & 0 & 0 & 0 & 0 & 0 & 0 & 0 & 0 & 0 & 0 & 0 \\
\hline $600-1200$ & 0 & 0 & 0 & 0 & 0 & 0 & 0 & 0 & 0 & 0 & 0 \\
\hline
\end{tabular}

Tabla 5. 4.f. Potencias calculadas de la señal en el dominio original obtenida de la línea axilar derecha basal para un flujo de $1.0 \mathrm{~L} / \mathrm{seg}$. 


\begin{tabular}{|c|c|c|c|c|c|c|c|c|c|c|c|}
\hline \multirow{4}{*}{ INTERVALOS } & \multicolumn{11}{|c|}{ MIT } \\
\hline & \multicolumn{4}{|c|}{ ZONA DE APNEA } & \multicolumn{7}{|c|}{ ZONA DE RESPIRACIÓN } \\
\hline & 0 & 3363 & 6847 & 10443 & 14274 & 18060 & 22058 & 26577 & 30603 & 34635 & 0 \\
\hline & 0 & 4819 & 8233 & 11829 & 15660 & 19446 & 23540 & 27963 & 32103 & 36021 & 0 \\
\hline $\begin{array}{l}\text { BANDAS DE } \\
\text { FRECUENCIA }\end{array}$ & \multicolumn{11}{|c|}{ SEÑAL ORIGINAL } \\
\hline $0-300$ & 0 & 629.99 & 694.77 & 773.43 & 835.83 & 993.38 & 800.77 & 823.79 & 982.81 & 695.78 & 0 \\
\hline $300-600$ & 0 & 89.826 & 74.08 & 93.569 & 82.697 & 88.881 & 86.318 & 81.02 & 84.47 & 74.266 & 0 \\
\hline $600-1200$ & 0 & 213.78 & 200.13 & 215.95 & 209.78 & 203.26 & 232.49 & 192.88 & 197.96 & 213.19 & 0 \\
\hline $\begin{array}{l}\text { BANDAS DE } \\
\text { FRECUENCIA }\end{array}$ & \multicolumn{11}{|c|}{ POTENCIAS DE LAS SEÑALES DE SONIDO RESPIRATORIO ESTIMADO SIN TW } \\
\hline $0-300$ & 0 & 388.09 & 341.87 & 347.48 & 613.27 & 545.07 & 566.35 & 423.19 & 652.69 & 380.03 & 0 \\
\hline $300-600$ & 0 & 17.163 & 9.6234 & 8.8153 & 21.422 & 27.842 & 21.625 & 35.597 & 51.949 & 25.057 & 0 \\
\hline $600-1200$ & 0 & 4.5496 & 2.806 & 2.6354 & 4.4375 & 7.8229 & 11.572 & 9.0174 & 16.917 & 10.274 & 0 \\
\hline $\begin{array}{l}\text { BANDAS DE } \\
\text { FRECUENCIA }\end{array}$ & \multicolumn{11}{|c|}{ POTENCIAS DE LAS SEÑALES DE SONIDO RESPIRATORIO ESTIMADO CON TW } \\
\hline $0-300$ & 0 & 385.01 & 373.02 & 333.46 & 628.22 & 590.71 & 606.16 & 346.72 & 805.34 & 400.54 & 0 \\
\hline $300-600$ & 0 & 57.604 & 25.197 & 14.047 & 30.485 & 53.066 & 35.282 & 42.176 & 88.879 & 35.686 & 0 \\
\hline $600-1200$ & 0 & 14.041 & 7.1086 & 6.8283 & 8.4192 & 15.691 & 17.625 & 15.531 & 30.61 & 20.283 & 0 \\
\hline $\begin{array}{l}\text { BANDAS DE } \\
\text { FRECUENCIA }\end{array}$ & \multicolumn{11}{|c|}{ POTENCIAS DE RUIDO CARDIACO ESTIMADO SIN TW } \\
\hline $0-300$ & 0 & 478.86 & 486.34 & 520.85 & 597.26 & 946.95 & 552.41 & 783.48 & 1012.9 & 546.32 & 0 \\
\hline $300-600$ & 0 & 15.31 & 8.538 & 7.4585 & 18.554 & 25.977 & 19.857 & 34.874 & 51.253 & 24.544 & 0 \\
\hline $600-1200$ & 0 & 4.3896 & 2.7297 & 2.4287 & 4.1712 & 7.7437 & 11.408 & 8.9442 & 16.947 & 10.299 & 0 \\
\hline $\begin{array}{l}\text { BANDAS DE } \\
\text { FRECUENCIA }\end{array}$ & \multicolumn{11}{|c|}{ POTENCIAS DE RUIDO CARDIACO ESTIMADO CON TW } \\
\hline 0-300 & 0 & 482.84 & 491.37 & 524.94 & 576.79 & 882.76 & 550.4 & 727.48 & 1090.7 & 542.46 & 0 \\
\hline $300-600$ & 0 & 57.284 & 24.977 & 13.142 & 28.402 & 52.104 & 33.184 & 41.059 & 88.49 & 35.313 & 0 \\
\hline $600-1200$ & 0 & 13.986 & 7.0741 & 6.7329 & 8.2784 & 15.648 & 17.556 & 15.46 & 30.652 & 20.263 & \\
\hline
\end{tabular}

Tabla 5. 4.g. Potencias calculadas de la señal en el dominio original obtenida de la zona de auscultación de la válvula mitral para un flujo de $1.0 \mathrm{~L} / \mathrm{seg}$.

\begin{tabular}{|c|c|c|c|c|c|c|c|c|c|c|c|}
\hline \multirow{4}{*}{ INTERVALOS } & \multicolumn{11}{|c|}{ TRI } \\
\hline & \multicolumn{4}{|c|}{ ZONA DE APNEA } & \multicolumn{7}{|c|}{ ZONA DE RESPIRACIÓN } \\
\hline & 251 & 3571 & 6931 & 10602 & 14310 & 18008 & 22102 & 26512 & 30894 & 34768 & 38997 \\
\hline & 831 & 4462 & 7801 & 11472 & 15271 & 18878 & 23026 & 27472 & 31786 & 35638 & 39993 \\
\hline $\begin{array}{l}\text { BANDAS DE } \\
\text { FRECUENCIA }\end{array}$ & \multicolumn{11}{|c|}{ SEÑAL ORIGINAL } \\
\hline $0-300$ & 889.18 & 1129.4 & 1427.8 & 1689.9 & 1930.4 & 2125.1 & 1603.2 & 1618.1 & 1854.2 & 1640.6 & 1719.3 \\
\hline $300-600$ & 36.844 & 59.384 & 61.117 & 50.163 & 57.37 & 53.086 & 61.711 & 61.222 & 71.79 & 48.4 & 77.943 \\
\hline $600-1200$ & 103.46 & 132.4 & 121.6 & 104.38 & 139.16 & 128.34 & 142.46 & 126.45 & 124.63 & 126.06 & 151.09 \\
\hline $\begin{array}{l}\text { BANDAS DE } \\
\text { FRECUENCIA }\end{array}$ & \multicolumn{11}{|c|}{ POTENCIAS DE LAS SEÑALES DE SONIDO RESPIRATORIO ESTIMADO SIN TW } \\
\hline $0-300$ & 602.89 & 634.95 & 394.49 & 1017 & 1170.6 & 1305.1 & 497.84 & 912.79 & 931.88 & 1115.6 & 823.37 \\
\hline $300-600$ & 9.0913 & 24.996 & 12.353 & 52.925 & 69.363 & 41.723 & 34.933 & 50.618 & 63.517 & 53.476 & 47.021 \\
\hline $600-1200$ & 1.3153 & 3.7458 & 4.0009 & 12.891 & 29.826 & 9.2619 & 16.604 & 26.723 & 19.306 & 14.245 & 22.114 \\
\hline $\begin{array}{l}\text { BANDAS DE } \\
\text { FRECUENCIA }\end{array}$ & \multicolumn{11}{|c|}{ POTENCIAS DE LAS SEÑALES DE SONIDO RESPIRATORIO ESTIMADO CON TW } \\
\hline $0-300$ & 583.63 & 627.32 & 440.26 & 955.48 & 1341.8 & 1322.8 & 553.96 & 883.03 & 1405.4 & 939.29 & 853.13 \\
\hline $300-600$ & 15.622 & 26.303 & 24.597 & 71.228 & 152.57 & 58.715 & 60.497 & 108.11 & 154.64 & 120.89 & 74.626 \\
\hline $600-1200$ & 3.6965 & 5.7451 & 9.5125 & 22.134 & 75.932 & 23.477 & 40.35 & 50.625 & 52.28 & 35.126 & 35.726 \\
\hline $\begin{array}{l}\text { BANDAS DE } \\
\text { FRECUENCIA }\end{array}$ & \multicolumn{11}{|c|}{ POTENCIAS DE RUIDO CARDIACO ESTIMADO SIN TW } \\
\hline $0-300$ & 317.24 & 963.58 & 1261.1 & 1463.4 & 1968.4 & 1581.4 & 1539.9 & 1470 & 1811 & 1739.2 & 1442.8 \\
\hline $300-600$ & 7.5524 & 22.382 & 14.761 & 51.945 & 64.893 & 39.839 & & & & 50.505 & 48.05 \\
\hline $600-1200$ & 1.1113 & 3.4203 & 4.0016 & 12.69 & 29.729 & 9.1552 & 16.437 & 26.733 & 19.276 & 14.025 & 22.027 \\
\hline $\begin{array}{l}\text { BANDAS DE } \\
\text { FRECUENCIA }\end{array}$ & \multicolumn{11}{|c|}{ POTENCIAS DE RUIDO CARDIACO ESTIMADO CON TW } \\
\hline $0-300$ & 294.01 & 917.75 & 1320.1 & 1365.9 & 2179.5 & 1608 & 1544.4 & 1476.5 & 2020.1 & 1629.3 & 1547 \\
\hline $300-600$ & 15.112 & 24.348 & 26.802 & 70.03 & 150.74 & 58.039 & 59.433 & 106.7 & 153.28 & 119.36 & 75.148 \\
\hline $600-1200$ & 3.6178 & 5.5306 & 9.5033 & 21.856 & 75.904 & 23.388 & 40.305 & 50.491 & 52.175 & 35.04 & 35.631 \\
\hline
\end{tabular}

Tabla 5. 4.h. Potencias calculadas de la señal en el dominio original obtenida de la zona de auscultación de la válvula tricúspide para un flujo de $1.0 \mathrm{~L} / \mathrm{seg}$. 


\begin{tabular}{|c|c|c|c|c|c|c|c|c|c|c|c|}
\hline \multirow{4}{*}{ INTERVALOS } & \multicolumn{11}{|c|}{ PUL } \\
\hline & \multicolumn{4}{|c|}{ ZONA DE APNEA } & \multicolumn{7}{|c|}{ ZONA DE RESPIRACIÓN } \\
\hline & 18 & 3690 & 0 & 0 & 14725 & 18141 & 22367 & 26752 & 31042 & 0 & 0 \\
\hline & 797 & 4342 & 0 & 0 & 15377 & 18855 & 23019 & 27404 & 31797 & 0 & 0 \\
\hline $\begin{array}{l}\text { BANDAS DE } \\
\text { FRECUENCIA }\end{array}$ & \multicolumn{11}{|c|}{ SEÑAL ORIGINAL } \\
\hline $0-300$ & 2594.7 & 2259.7 & 0 & 0 & 19744 & 7909.7 & 2886.6 & 2960.5 & 10293 & 0 & 0 \\
\hline $300-600$ & 74.116 & 58.552 & 0 & 0 & 100.49 & 100.04 & 75.526 & 122.78 & 67.645 & 0 & 0 \\
\hline $600-1200$ & 137.25 & 107.86 & 0 & 0 & 120.1 & 124.14 & 121.76 & 114.29 & 135.46 & 0 & 0 \\
\hline $\begin{array}{l}\text { BANDAS DE } \\
\text { FRECUENCIA }\end{array}$ & \multicolumn{11}{|c|}{ POTENCIAS DE LAS SEÑALES DE SONIDO RESPIRATORIO ESTIMADO SIN TW } \\
\hline $0-300$ & 1872.1 & 1594.4 & 0 & 0 & 14530 & 7818.1 & 2744.9 & 1573 & 6585.4 & 0 & 0 \\
\hline $300-600$ & 39.928 & 28.702 & 0 & 0 & 54.932 & 63.476 & 35.362 & 45.193 & 111.78 & 0 & 0 \\
\hline $600-1200$ & 17.386 & 7.2277 & 0 & 0 & 19.481 & 28.674 & 18.724 & 20.774 & 77.636 & 0 & 0 \\
\hline $\begin{array}{l}\text { BANDAS DE } \\
\text { FRECUENCIA }\end{array}$ & \multicolumn{11}{|c|}{ POTENCIAS DE LAS SEÑALES DE SONIDO RESPIRATORIO ESTIMADO CON TW } \\
\hline $0-300$ & 1889.7 & 1593.7 & 0 & 0 & 14424 & 7890.7 & 2749 & 1499.3 & 6761.1 & 0 & 0 \\
\hline $300-600$ & 40.381 & 31.354 & 0 & 0 & 156.68 & 186.65 & 97.979 & 99.326 & 141.12 & 0 & 0 \\
\hline $600-1200$ & 19.947 & 6.1974 & 0 & 0 & 177.14 & 264.22 & 164.43 & 162.5 & 58.343 & 0 & 0 \\
\hline $\begin{array}{l}\text { BANDAS DE } \\
\text { FRECUENCIA }\end{array}$ & \multicolumn{11}{|c|}{ POTENCIAS DE RUIDO CARDIACO ESTIMADO SIN TW } \\
\hline $0-300$ & 769.83 & 552.98 & 0 & 0 & 1960.1 & 1333.1 & 579.67 & 1113.8 & 2139.1 & 0 & 0 \\
\hline $300-600$ & 17.267 & 9.2226 & 0 & 0 & 28.717 & 39.029 & 14.971 & 32.845 & 111.95 & 0 & 0 \\
\hline $600-1200$ & 13.05 & 4.3138 & 0 & 0 & 17.425 & 23.006 & 17.139 & 18.717 & 77.165 & 0 & 0 \\
\hline $\begin{array}{l}\text { BANDAS DE } \\
\text { FRECUENCIA }\end{array}$ & \multicolumn{11}{|c|}{ POTENCIAS DE RUIDO CARDIACO ESTIMADO CON TW } \\
\hline 0-300 & 779.6 & 554.54 & 0 & 0 & 2003.6 & 1410.4 & 615.71 & 1175.4 & 2066.2 & 0 & 0 \\
\hline $300-600$ & 22.185 & 11.353 & 0 & 0 & 133.25 & 168.57 & 83.173 & 94.566 & 138.67 & 0 & 0 \\
\hline $600-1200$ & 16.214 & 3.4412 & 0 & 0 & 176.49 & 259.78 & 164.52 & 162.78 & 58.265 & 0 & \\
\hline
\end{tabular}

Tabla 5. 4.i. Potencias calculadas de la señal en el dominio original obtenida de la zona de auscultación de la válvula pulmonar para un flujo de $1.0 \mathrm{~L} / \mathrm{seg}$.

\begin{tabular}{|c|c|c|c|c|c|c|c|c|c|c|c|}
\hline \multirow{4}{*}{ INTERVALOS } & \multicolumn{11}{|c|}{ AOR } \\
\hline & \multicolumn{4}{|c|}{ ZONA DE APNEA } & \multicolumn{7}{|c|}{ ZONA DE RESPIRACIÓN } \\
\hline & 96 & 3710 & 7061 & 10555 & 14446 & 18112 & 22096 & 26757 & 31112 & 34845 & 39177 \\
\hline & 1046 & 4392 & 8011 & 11505 & 15128 & 19062 & 23046 & 27439 & 31873 & 35795 & 40127 \\
\hline \multicolumn{12}{|l|}{$\begin{array}{l}\text { BANDAS DE } \\
\text { FRECUENCIA }\end{array}$} \\
\hline $0-300$ & 1643.8 & 1011 & 1669.8 & 1644.6 & 1369.6 & 2271.8 & 2332.4 & 1469.9 & 2055.7 & 1553.2 & 2197.1 \\
\hline $300-600$ & 56.912 & 42.62 & 61.31 & 63.091 & 76.996 & 76.634 & 58.654 & 89.108 & 44.382 & 60.836 & 131.36 \\
\hline $600-1200$ & 133.52 & 97.728 & 136.28 & 148.04 & 102.15 & 128.23 & 121.45 & 100.08 & 107.86 & 114.21 & 142.37 \\
\hline $\begin{array}{l}\text { BANDAS DE } \\
\text { FRECUENCIA }\end{array}$ & \multicolumn{11}{|c|}{ POTENCIAS DE LAS SEÑALES DE SONIDO RESPIRATORIO ESTIMADO SIN TW } \\
\hline $0-300$ & 1124.4 & 848.64 & 1212.4 & 1344 & 992.06 & 1548.1 & 1982.6 & 1032.9 & 1405.8 & 1214.8 & 1349.5 \\
\hline $300-600$ & 37.286 & 45.381 & 38.976 & 44.543 & 55.795 & 46.342 & 53.904 & 45.322 & 48.359 & 131.05 & 50.659 \\
\hline $600-1200$ & 13.83 & 8.1774 & 10.817 & 8.6013 & 11.567 & 18.126 & 23.599 & 16.17 & 29.972 & 66.367 & 19.194 \\
\hline $\begin{array}{l}\text { BANDAS DE } \\
\text { FRECUENCIA }\end{array}$ & \multicolumn{11}{|c|}{ POTENCIAS DE LAS SEÑALES DE SONIDO RESPIRATORIO ESTIMADO CON TW } \\
\hline $0-300$ & 1150.6 & 867.74 & 1272.2 & 1345.7 & 1141.1 & 1534.4 & 1972.8 & 1005.3 & 1444.4 & 1333.7 & 1418.5 \\
\hline $300-600$ & 47.243 & 80.596 & 51.668 & 50.216 & 63.658 & 61.734 & 101.55 & 49.29 & 49.297 & 358.8 & 103.89 \\
\hline $600-1200$ & 12.87 & 29.44 & 13.812 & 16.991 & 22.846 & 32.175 & 57.711 & 33.632 & 42.909 & 142.79 & 48.277 \\
\hline $\begin{array}{l}\text { BANDAS DE } \\
\text { FRECUENCIA }\end{array}$ & \multicolumn{11}{|c|}{ POTENCIAS DE RUIDO CARDIACO ESTIMADO SIN TW } \\
\hline $0-300$ & 931.2 & 457.83 & 579.74 & 609.7 & 502.51 & 936.68 & 1047.7 & 519.88 & 802.87 & 1400.4 & 1242 \\
\hline $300-600$ & 18.703 & 32.422 & 12.488 & 13.184 & 28.901 & 26.057 & 48.056 & 29.863 & 39.942 & 122.79 & 29.096 \\
\hline $600-1200$ & 9.9811 & 4.2886 & 4.7116 & 4.9679 & 9.5548 & 14.682 & 22.482 & 15.264 & 28.709 & 65.242 & 16.78 \\
\hline $\begin{array}{l}\text { BANDAS DE } \\
\text { FRECUENCIA }\end{array}$ & \multicolumn{11}{|c|}{ POTENCIAS DE RUIDO CARDIACO ESTIMADO CON TW } \\
\hline 0-300 & 874.29 & 517.89 & 663.39 & 659.38 & 644.59 & 920.2 & 1078.7 & 568.57 & 733.81 & 1471.9 & 1276.9 \\
\hline $300-600$ & 29.749 & 71.97 & 27.79 & 20.763 & 46.117 & 45.231 & 97.747 & 40.178 & 40.172 & 355.07 & 84.417 \\
\hline $600-1200$ & 10.346 & 26.723 & 8.224 & 14.453 & 21.972 & 29.72 & 57.598 & 33.471 & 42.404 & 142.35 & 46.736 \\
\hline
\end{tabular}

Tabla 5. 4.j. Potencias calculadas de la señal en el dominio original obtenida de la zona de auscultación de la válvula aórtica para un flujo de 1.0L/seg. 


\begin{tabular}{|c|c|c|c|c|c|c|c|c|c|c|c|}
\hline \multirow{4}{*}{ INTERVALOS } & \multicolumn{11}{|c|}{ PRE } \\
\hline & \multicolumn{4}{|c|}{ ZONA DE APNEA } & \multicolumn{7}{|c|}{ ZONA DE RESPIRACIÓN } \\
\hline & 65 & 3454 & 6952 & 10766 & 14440 & 18113 & 22166 & 0 & 30936 & 0 & 39097 \\
\hline & 1030 & 4419 & 7927 & 11636 & 15310 & 18983 & 23245 & 0 & 31817 & 0 & 40062 \\
\hline $\begin{array}{l}\text { BANDAS DE } \\
\text { FRECUENCIA }\end{array}$ & \multicolumn{11}{|c|}{ SEÑAL ORIGINAL } \\
\hline $0-300$ & 686.85 & 713.25 & 875.64 & 827.34 & 799.33 & 866.31 & 1203.7 & 0 & 977.36 & 0 & 822.93 \\
\hline $300-600$ & 47.308 & 56.21 & 55.46 & 51.683 & 45.842 & 63.729 & 72.486 & 0 & 47.832 & 0 & 55.389 \\
\hline $600-1200$ & 145 & 158.05 & 160.48 & 138.24 & 147.19 & 136.4 & 170.71 & 0 & 142.72 & 0 & 135.88 \\
\hline $\begin{array}{l}\text { BANDAS DE } \\
\text { FRECUENCIA }\end{array}$ & \multicolumn{11}{|c|}{ POTENCIAS DE LAS SEÑALES DE SONIDO RESPIRATORIO ESTIMADO SIN TW } \\
\hline $0-300$ & 559.73 & 414.11 & 533.9 & 505.74 & 496.16 & 516.44 & 1275.5 & 0 & 619.18 & 0 & 794.88 \\
\hline $300-600$ & 16.666 & 7.9092 & 19.159 & 4.8838 & 12.51 & 4.5545 & 32.458 & 0 & 14.602 & 0 & 22.06 \\
\hline $600-1200$ & 1.9085 & 0.9557 & 3.7278 & 0.77244 & 1.5997 & 0.66666 & 9.075 & 0 & 1.9315 & 0 & 6.8361 \\
\hline $\begin{array}{l}\text { BANDAS DE } \\
\text { FRECUENCIA }\end{array}$ & \multicolumn{11}{|c|}{ POTENCIAS DE LAS SEÑALES DE SONIDO RESPIRATORIO ESTIMADO CON TW } \\
\hline $0-300$ & 498.72 & 413.98 & 558.55 & 505.67 & 527.89 & 518.67 & 1134 & 0 & 718.33 & 0 & 639.85 \\
\hline $300-600$ & 14.177 & 8.0603 & 21.101 & 4.4128 & 20.866 & 11.503 & 46.812 & 0 & 17.391 & 0 & 25.906 \\
\hline $600-1200$ & 4.2404 & 0.86028 & 7.2695 & 0.86371 & 4.6956 & 3.7563 & 15.887 & 0 & 5.5847 & 0 & 11.321 \\
\hline $\begin{array}{l}\text { BANDAS DE } \\
\text { FRECUENCIA }\end{array}$ & \multicolumn{11}{|c|}{ POTENCIAS DE RUIDO CARDIACO ESTIMADO SIN TW } \\
\hline $0-300$ & 544.84 & 597.63 & 684.42 & 461.64 & 500.43 & 116.7 & 860.54 & 0 & 579.38 & 0 & 604.11 \\
\hline $300-600$ & 13.915 & 5.1042 & 16.348 & 3.4834 & 8.6523 & 2.489 & 32.113 & 0 & 12.58 & 0 & 21.175 \\
\hline $600-1200$ & 1.6542 & 0.77534 & 3.2755 & 0.63658 & 1.353 & 0.53593 & 8.9492 & 0 & 1.675 & 0 & 6.7261 \\
\hline $\begin{array}{l}\text { BANDAS DE } \\
\text { FRECUENCIA }\end{array}$ & \multicolumn{11}{|c|}{ POTENCIAS DE RUIDO CARDIACO ESTIMADO CON TW } \\
\hline $0-300$ & 555.1 & 599.47 & 679.39 & 461.4 & 511.06 & 119.81 & 784.49 & 0 & 507.33 & 0 & 568.86 \\
\hline $300-600$ & 11.67 & 4.9731 & 17.214 & 3.4242 & 16.486 & 9.5558 & 44.212 & 0 & 15.943 & 0 & 25.487 \\
\hline $600-1200$ & 4.0628 & 0.73109 & 6.8456 & 0.76642 & 4.4598 & 3.6682 & 15.697 & 0 & 5.5347 & 0 & 11.333 \\
\hline
\end{tabular}

Tabla 5. 4.k. Potencias calculadas de la señal en el dominio original obtenida de la zona de auscultación precordial promedio para un flujo de $1.0 \mathrm{~L} / \mathrm{seg}$. 
Tabla 5. 5 Potencias calculadas de la señal en el dominio original obtenida un flujo de 1.5L/seg.

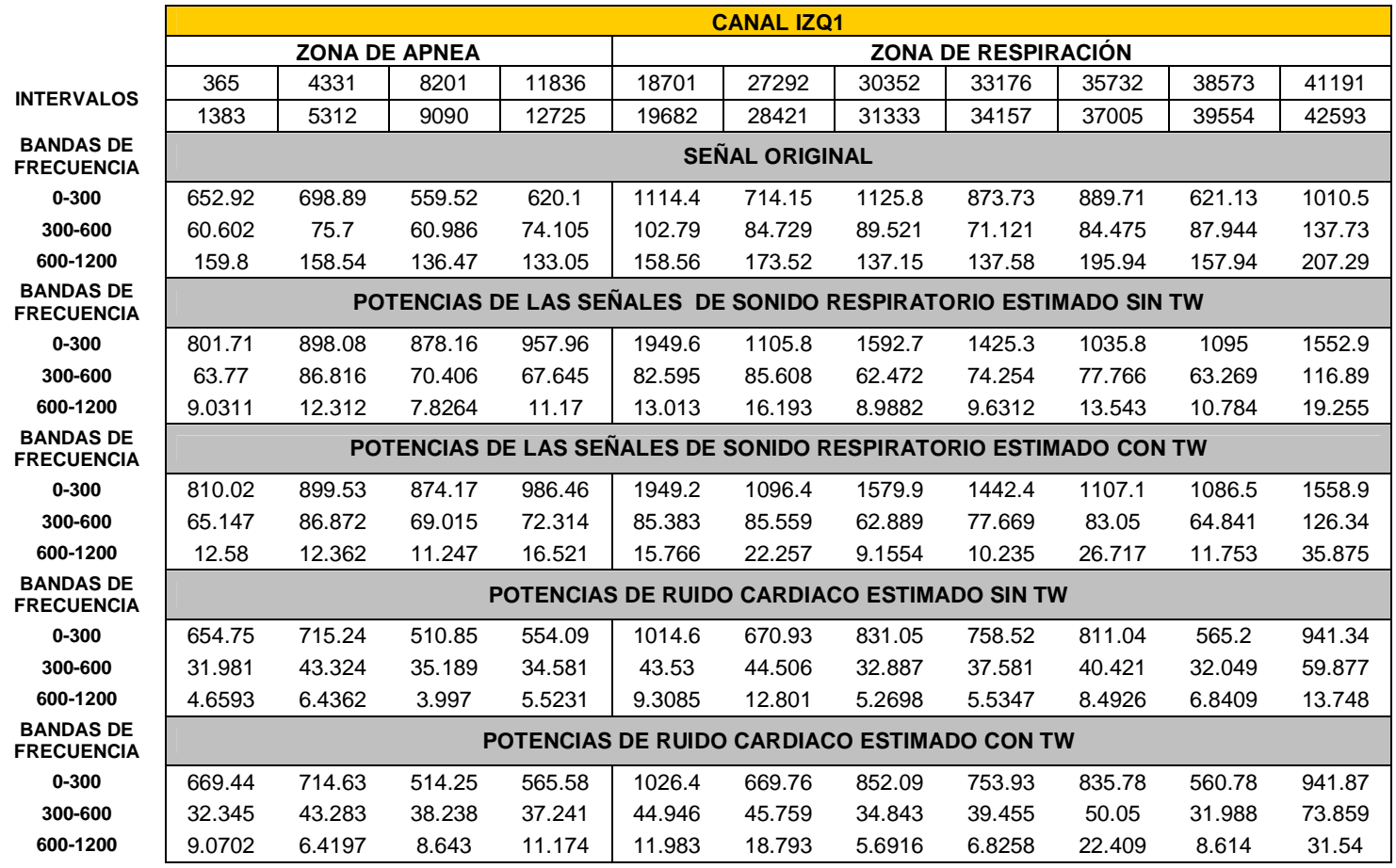

Tabla 5. 5.a. Potencias calculadas de la señal en el dominio original obtenida de la línea axilar izquierda apical para un flujo de $1.5 \mathrm{~L} / \mathrm{seg}$.

\begin{tabular}{|c|c|c|c|c|c|c|c|c|c|c|c|}
\hline \multirow{4}{*}{ INTERVALOS } & \multicolumn{11}{|c|}{ CANAL IZQ2 } \\
\hline & \multicolumn{4}{|c|}{ ZONA DE APNEA } & \multicolumn{7}{|c|}{ ZONA DE RESPIRACIÓN } \\
\hline & 287 & 4216 & 8091 & 11934 & 18672 & 27450 & 30208 & 33196 & 35800 & 38524 & 0 \\
\hline & 1716 & 5645 & 9520 & 13363 & 20224 & 28879 & 31637 & 34625 & 37229 & 39590 & 0 \\
\hline $\begin{array}{l}\text { BANDAS DE } \\
\text { FRECUENCIA }\end{array}$ & \multicolumn{11}{|c|}{ SEÑAL ORIGINAL } \\
\hline $0-300$ & 1652.3 & 1732.6 & 1432.9 & 1671.5 & 4884.5 & 2066 & 2775.9 & 4061.7 & 2885.3 & 1365.1 & 0 \\
\hline $300-600$ & 76.774 & 96.121 & 82.077 & 161.76 & 202.67 & 101.44 & 191.96 & 101.24 & 99.54 & 99.142 & 0 \\
\hline $600-1200$ & 176.33 & 202.75 & 201.04 & 184.76 & 200.33 & 189.29 & 189.1 & 188.18 & 182.3 & 149.19 & 0 \\
\hline $\begin{array}{c}\text { BANDAS DE } \\
\text { FRECUENCIA }\end{array}$ & \multicolumn{11}{|c|}{ POTENCIAS DE LAS SEÑALES DE SONIDO RESPIRATORIO ESTIMADO SIN TW } \\
\hline $0-300$ & 613.71 & 698.03 & 717.39 & 1406.1 & 4780.8 & 1476.4 & 2260.3 & 2659.7 & 1642.1 & 2090.4 & 0 \\
\hline $300-600$ & 47.008 & 47.431 & 39.615 & 55.462 & 939.36 & 111.87 & 382.34 & 392.53 & 129.64 & 223.16 & 0 \\
\hline $600-1200$ & 14.141 & 11.077 & 6.5625 & 12.213 & 1265.9 & 65.744 & 596.13 & 536.09 & 66.298 & 86.23 & 0 \\
\hline $\begin{array}{l}\text { BANDAS DE } \\
\text { FRECUENCIA }\end{array}$ & \multicolumn{11}{|c|}{ POTENCIAS DE LAS SEÑALES DE SONIDO RESPIRATORIO ESTIMADO CON TW } \\
\hline $0-300$ & 637.12 & 696.91 & 740.41 & 1433.9 & 6739 & 1358 & 2808.3 & 2742.9 & 1728.4 & 2324.1 & 0 \\
\hline $300-600$ & 48.291 & 47.722 & 57.238 & 57.95 & 3025.5 & 97.233 & 1540.8 & 1341 & 224.26 & 902.53 & 0 \\
\hline $600-1200$ & 15.035 & 11.489 & 15.177 & 15.127 & 3800.7 & 52.5 & 2607.6 & 2277.8 & 114.14 & 989.77 & 0 \\
\hline $\begin{array}{l}\text { BANDAS DE } \\
\text { FRECUENCIA }\end{array}$ & \multicolumn{11}{|c|}{ POTENCIAS DE RUIDO CARDIACO ESTIMADO SIN TW } \\
\hline $0-300$ & 1209.5 & 1291.3 & 880.2 & 915.37 & 5791.5 & 1475.3 & 1774.2 & 3530.1 & 2584.6 & 1300.3 & 0 \\
\hline $300-600$ & 14.926 & 17.605 & 6.7683 & 22.947 & 935.53 & 83.997 & 381.02 & 387.21 & 100.34 & 218.98 & 0 \\
\hline $600-1200$ & 9.8177 & 5.5472 & 2.085 & 8.5363 & 1263.7 & 62.006 & 595.91 & 535.68 & 63.511 & 85.93 & 0 \\
\hline $\begin{array}{l}\text { BANDAS DE } \\
\text { FRECUENCIA }\end{array}$ & \multicolumn{11}{|c|}{ POTENCIAS DE RUIDO CARDIACO ESTIMADO CON TW } \\
\hline $0-300$ & 1210 & 1289.6 & 919.26 & 970.88 & 7800.9 & 1389.2 & 2203.1 & 3941.7 & 2798.9 & 1595.7 & 0 \\
\hline $300-600$ & 19.703 & 18.5 & 34.509 & 28.597 & 3010.3 & 80.108 & 1539.3 & 1336.5 & 210.17 & 895.56 & 0 \\
\hline $600-1200$ & 11.358 & 5.9354 & 12.979 & 13.502 & 3800.3 & 50.19 & 2607.7 & 2278 & 112.93 & 990.2 & 0 \\
\hline
\end{tabular}

Tabla 5. 5.b. Potencias calculadas de la señal en el dominio original obtenida de la línea axilar izquierda media para un flujo de $1.5 \mathrm{~L} / \mathrm{seg}$. 


\begin{tabular}{|c|c|c|c|c|c|c|c|c|c|c|c|}
\hline \multirow{4}{*}{ INTERVALOS } & \multicolumn{11}{|c|}{ CANAL IZQ3 } \\
\hline & \multicolumn{4}{|c|}{ ZONA DE APNEA } & \multicolumn{7}{|c|}{ ZONA DE RESPIRACIÓN } \\
\hline & 402 & 4378 & 8161 & 11821 & 18681 & 0 & 30208 & 33041 & 35786 & 38742 & 41417 \\
\hline & 1844 & 5733 & 9516 & 13176 & 20036 & 0 & 31563 & 34396 & 37141 & 39453 & 42772 \\
\hline $\begin{array}{l}\text { BANDAS DE } \\
\text { FRECUENCIA }\end{array}$ & \multicolumn{11}{|c|}{ SEÑAL ORIGINAL } \\
\hline $0-300$ & 831.31 & 824.1 & 796.42 & 731.66 & 2015 & 0 & 1714 & 1383 & 1540.7 & 559.34 & 1421.7 \\
\hline $300-600$ & 88.465 & 98.534 & 81.94 & 127.3 & 128.29 & 0 & 114.62 & 97.937 & 97.061 & 54.629 & 116.72 \\
\hline $600-1200$ & 216.86 & 233.97 & 201.15 & 215.27 & 229.23 & 0 & 190.63 & 195.42 & 201.95 & 107.52 & 220.19 \\
\hline $\begin{array}{l}\text { BANDAS DE } \\
\text { FRECUENCIA }\end{array}$ & \multicolumn{11}{|c|}{ POTENCIAS DE LAS SEÑALES DE SONIDO RESPIRATORIO ESTIMADO SIN TW } \\
\hline 0-300 & 353.6 & 444.63 & 489.17 & 457.95 & 1463.2 & 0 & 1445.4 & 1127.4 & 832.77 & 566.03 & 988.21 \\
\hline $300-600$ & 44.618 & 43.113 & 38.7 & 39.917 & 59.347 & 0 & 50.371 & 41.167 & 43.914 & 42.849 & 79.884 \\
\hline $600-1200$ & 14.466 & 10.867 & 9.2969 & 6.8329 & 16.435 & 0 & 7.1714 & 6.0366 & 9.7464 & 13.034 & 23.313 \\
\hline $\begin{array}{l}\text { BANDAS DE } \\
\text { FRECUENCIA }\end{array}$ & \multicolumn{11}{|c|}{ POTENCIAS DE LAS SEÑALES DE SONIDO RESPIRATORIO ESTIMADO CON TW } \\
\hline $0-300$ & 352.79 & 437.25 & 494.52 & 457.58 & 1549.2 & 0 & 1475.6 & 1128.5 & 849.6 & 601.37 & 1002.3 \\
\hline $300-600$ & 56.168 & 62.261 & 51.095 & 39.83 & 75.934 & 0 & 65.286 & 42.544 & 92.924 & 74.431 & 174.13 \\
\hline $600-1200$ & 26.514 & 27.165 & 21.874 & 6.8147 & 25.346 & 0 & 14.671 & 10.246 & 53.491 & 22.767 & 71.042 \\
\hline $\begin{array}{l}\text { BANDAS DE } \\
\text { FRECUENCIA }\end{array}$ & \multicolumn{11}{|c|}{ POTENCIAS DE RUIDO CARDIACO ESTIMADO SIN TW } \\
\hline $0-300$ & 719.81 & 680.48 & 521.03 & 522.39 & 1188.8 & 0 & 582.78 & 776.8 & 1016.8 & 388.21 & 836.61 \\
\hline $300-600$ & 14.74 & 14.928 & 9.9573 & 4.0288 & 32.296 & 0 & 9.2703 & 12.399 & 14.915 & 34.246 & 48.263 \\
\hline $600-1200$ & 11.176 & 7.6357 & 6.6462 & 3.2478 & 13.847 & 0 & 3.0661 & 2.2048 & 5.6417 & 11.934 & 20.657 \\
\hline $\begin{array}{l}\text { BANDAS DE } \\
\text { FRECUENCIA }\end{array}$ & \multicolumn{11}{|c|}{ POTENCIAS DE RUIDO CARDIACO ESTIMADO CON TW } \\
\hline $0-300$ & 710.63 & 668.92 & 523.48 & 524.02 & 1273.3 & 0 & 581.22 & 746.7 & 1033.4 & 378.48 & 882.8 \\
\hline $300-600$ & 35.009 & 39.33 & 28.13 & 4.8493 & 53.974 & 0 & 30.271 & 19.784 & 75.335 & 69.316 & 148.63 \\
\hline $600-1200$ & 24.161 & 25.381 & 21.273 & 3.3356 & 23.144 & 0 & 12.025 & 6.8872 & 52.304 & 22.35 & 69.821 \\
\hline
\end{tabular}

Tabla 5. 5.c. Potencias calculadas de la señal en el dominio original obtenida de la línea axilar izquierda basal para un flujo de $1.5 \mathrm{~L} / \mathrm{seg}$.

\begin{tabular}{|c|c|c|c|c|c|c|c|c|c|c|c|}
\hline \multirow{4}{*}{ INTERVALOS } & \multicolumn{11}{|c|}{ DER1 } \\
\hline & \multicolumn{4}{|c|}{ APNEA } & \multicolumn{7}{|c|}{ ZONA DE RESPIRACIÓN } \\
\hline & 0 & 0 & 0 & 0 & 0 & 0 & 0 & 0 & 0 & 0 & 0 \\
\hline & 0 & 0 & 0 & 0 & 0 & 0 & 0 & 0 & 0 & 0 & 0 \\
\hline $\begin{array}{l}\text { BANDAS DE } \\
\text { FRECUENCIA }\end{array}$ & \multicolumn{11}{|c|}{ SEÑAL ORIGINAL } \\
\hline $0-300$ & 0 & 0 & 0 & 0 & 0 & 0 & 0 & 0 & 0 & 0 & 0 \\
\hline $300-600$ & 0 & 0 & 0 & 0 & 0 & 0 & 0 & 0 & 0 & 0 & 0 \\
\hline $600-1200$ & 0 & 0 & 0 & 0 & 0 & 0 & 0 & 0 & 0 & 0 & 0 \\
\hline $\begin{array}{l}\text { BANDAS DE } \\
\text { FRECUENCIA }\end{array}$ & \multicolumn{11}{|c|}{ POTENCIAS DE LAS SEÑALES DE SONIDO RESPIRATORIO ESTIMADO SIN TW } \\
\hline $0-300$ & 0 & 0 & 0 & 0 & 0 & 0 & 0 & 0 & 0 & 0 & 0 \\
\hline $300-600$ & 0 & 0 & 0 & 0 & 0 & 0 & 0 & 0 & 0 & 0 & 0 \\
\hline $600-1200$ & 0 & 0 & 0 & 0 & 0 & 0 & 0 & 0 & 0 & 0 & 0 \\
\hline $\begin{array}{l}\text { BANDAS DE } \\
\text { FRECUENCIA }\end{array}$ & \multicolumn{11}{|c|}{ POTENCIAS DE LAS SEÑALES DE SONIDO RESPIRATORIO ESTIMADO CON TW } \\
\hline $0-300$ & 0 & 0 & 0 & 0 & 0 & 0 & 0 & 0 & 0 & 0 & 0 \\
\hline $300-600$ & 0 & 0 & 0 & 0 & 0 & 0 & 0 & 0 & 0 & 0 & 0 \\
\hline $600-1200$ & 0 & 0 & 0 & 0 & 0 & 0 & 0 & 0 & 0 & 0 & 0 \\
\hline $\begin{array}{l}\text { BANDAS DE } \\
\text { FRECUENCIA }\end{array}$ & \multicolumn{11}{|c|}{ POTENCIAS DE RUIDO CARDIACO ESTIMADO SIN TW } \\
\hline $0-300$ & 0 & 0 & 0 & 0 & 0 & 0 & 0 & 0 & 0 & 0 & 0 \\
\hline $300-600$ & 0 & 0 & 0 & 0 & 0 & 0 & 0 & 0 & 0 & 0 & 0 \\
\hline $600-1200$ & 0 & 0 & 0 & 0 & 0 & 0 & 0 & 0 & 0 & 0 & 0 \\
\hline $\begin{array}{l}\text { BANDAS DE } \\
\text { FRECUENCIA }\end{array}$ & \multicolumn{11}{|c|}{ POTENCIAS DE RUIDO CARDIACO ESTIMADO CON TW } \\
\hline $0-300$ & 0 & 0 & 0 & 0 & 0 & 0 & 0 & 0 & 0 & 0 & 0 \\
\hline $300-600$ & 0 & 0 & 0 & 0 & 0 & 0 & 0 & 0 & 0 & 0 & 0 \\
\hline $600-1200$ & 0 & 0 & 0 & 0 & 0 & 0 & 0 & 0 & 0 & 0 & 0 \\
\hline
\end{tabular}

Tabla 5. 5.d. Potencias calculadas de la señal en el dominio original obtenida de la línea axilar derecha apical para un flujo de $1.5 \mathrm{~L} / \mathrm{seg}$. 


\begin{tabular}{|c|c|c|c|c|c|c|c|c|c|c|c|}
\hline \multirow{4}{*}{ INTERVALOS } & \multicolumn{11}{|c|}{ DER2 } \\
\hline & \multicolumn{4}{|c|}{ APNEA } & \multicolumn{7}{|c|}{ ZONA DE RESPIRACIÓN } \\
\hline & 372 & 4355 & 8322 & 12083 & 19490 & 0 & 30381 & 33271 & 0 & 0 & 41604 \\
\hline & 1648 & 5631 & 9598 & 13367 & 20766 & 0 & 31657 & 34090 & 0 & 0 & 42884 \\
\hline \multicolumn{12}{|l|}{$\begin{array}{l}\text { BANDAS DE } \\
\text { FRECUENCIA }\end{array}$} \\
\hline $0-300$ & 479.78 & 504.54 & 475.32 & 523.65 & 1611.4 & 0 & 1164.3 & 1353 & 0 & 0 & 1390.5 \\
\hline $300-600$ & 82.42 & 87.906 & 92.621 & 115.51 & 107.06 & 0 & 168.3 & 67.19 & 0 & 0 & 143.53 \\
\hline $600-1200$ & 198.23 & 217.64 & 203.36 & 188.22 & 195.68 & 0 & 205.97 & 142.18 & 0 & 0 & 205.57 \\
\hline $\begin{array}{l}\text { BANDAS DE } \\
\text { FRECUENCIA }\end{array}$ & \multicolumn{11}{|c|}{ POTENCIAS DE LAS SEÑALES DE SONIDO RESPIRATORIO ESTIMADO SIN TW } \\
\hline $0-300$ & 642.69 & 745.09 & 572.56 & 612.06 & 1230.7 & 0 & 1229.1 & 977.21 & 0 & 0 & 1634 \\
\hline $300-600$ & 33.361 & 41.634 & 35.783 & 48.334 & 33.999 & 0 & 42.122 & 30.677 & 0 & 0 & 47.39 \\
\hline $600-1200$ & 5.1805 & 7.1152 & 4.9275 & 6.2298 & 4.2049 & 0 & 5.4681 & 4.1591 & 0 & 0 & 6.1811 \\
\hline $\begin{array}{l}\text { BANDAS DE } \\
\text { FRECUENCIA }\end{array}$ & \multicolumn{11}{|c|}{ POTENCIAS DE LAS SEÑALES DE SONIDO RESPIRATORIO ESTIMADO CON TW } \\
\hline $0-300$ & 642.69 & 745.09 & 572.56 & 612.06 & 1230.7 & 0 & 1229.1 & 977.21 & 0 & 0 & 1634 \\
\hline $300-600$ & 33.361 & 41.634 & 35.783 & 48.334 & 33.999 & 0 & 42.122 & 30.677 & 0 & 0 & 47.39 \\
\hline $600-1200$ & 5.1805 & 7.1152 & 4.9275 & 6.2298 & 4.2049 & 0 & 5.4681 & 4.1591 & 0 & 0 & 6.1811 \\
\hline $\begin{array}{l}\text { BANDAS DE } \\
\text { FRECUENCIA }\end{array}$ & \multicolumn{11}{|c|}{ POTENCIAS DE RUIDO CARDIACO ESTIMADO SIN TW } \\
\hline 0-300 & 345.82 & 373.18 & 328.05 & 421 & 429.24 & 0 & 459.63 & 332.8 & 0 & 0 & 619.21 \\
\hline $300-600$ & 8.2712 & 3.8365 & 5.6646 & 11.21 & 8.6054 & 0 & 18.682 & 6.0852 & 0 & 0 & 21.551 \\
\hline $600-1200$ & 3.6749 & 1.3588 & 2.508 & 3.7793 & 5.0828 & 0 & 13.544 & 5.4845 & 0 & 0 & 13.774 \\
\hline $\begin{array}{l}\text { BANDAS DE } \\
\text { FRECUENCIA }\end{array}$ & \multicolumn{11}{|c|}{ POTENCIAS DE RUIDO CARDIACO ESTIMADO CON TW } \\
\hline $0-300$ & 347.74 & 372.44 & 352.05 & 380.99 & 427.23 & 0 & 448.3 & 359.04 & 0 & 0 & 646.77 \\
\hline $300-600$ & 7.712 & 5.2369 & 29.177 & 11.65 & 16.502 & 0 & 24.917 & 32.39 & 0 & 0 & 59.659 \\
\hline $600-1200$ & 3.7089 & 2.6465 & 13.39 & 5.4279 & 10.36 & 0 & 17.324 & 19.938 & 0 & 0 & 46.62 \\
\hline
\end{tabular}

Tabla 5. 5.e. Potencias calculadas de la señal en el dominio original obtenida de la línea axilar derecha media para un flujo de $1.5 \mathrm{~L} / \mathrm{seg}$.

\begin{tabular}{|c|c|c|c|c|c|c|c|c|c|c|c|}
\hline \multirow{4}{*}{ INTERVALOS } & \multicolumn{11}{|c|}{ DER3 } \\
\hline & \multicolumn{4}{|c|}{ APNEA } & \multicolumn{7}{|c|}{ ZONA DE RESPIRACIÓN } \\
\hline & 0 & 0 & 0 & 0 & 0 & 0 & 0 & 0 & 0 & 0 & 0 \\
\hline & 0 & 0 & 0 & 0 & 0 & 0 & 0 & 0 & 0 & 0 & 0 \\
\hline $\begin{array}{l}\text { BANDAS DE } \\
\text { FRECUENCIA }\end{array}$ & \multicolumn{11}{|c|}{ SEÑAL ORIGINAL } \\
\hline $0-300$ & 0 & 0 & 0 & 0 & 0 & 0 & 0 & 0 & 0 & 0 & 0 \\
\hline $300-600$ & 0 & 0 & 0 & 0 & 0 & 0 & 0 & 0 & 0 & 0 & 0 \\
\hline $600-1200$ & 0 & 0 & 0 & 0 & 0 & 0 & 0 & 0 & 0 & 0 & 0 \\
\hline $\begin{array}{l}\text { BANDAS DE } \\
\text { FRECUENCIA }\end{array}$ & \multicolumn{11}{|c|}{ POTENCIAS DE LAS SEÑALES DE SONIDO RESPIRATORIO ESTIMADO SIN TW } \\
\hline $0-300$ & 0 & 0 & 0 & 0 & 0 & 0 & 0 & 0 & 0 & 0 & 0 \\
\hline $300-600$ & 0 & 0 & 0 & 0 & 0 & 0 & 0 & 0 & 0 & 0 & 0 \\
\hline $600-1200$ & 0 & 0 & 0 & 0 & 0 & 0 & 0 & 0 & 0 & 0 & 0 \\
\hline $\begin{array}{l}\text { BANDAS DE } \\
\text { FRECUENCIA }\end{array}$ & \multicolumn{11}{|c|}{ POTENCIAS DE LAS SEÑALES DE SONIDO RESPIRATORIO ESTIMADO CON TW } \\
\hline $0-300$ & 0 & 0 & 0 & 0 & 0 & 0 & 0 & 0 & 0 & 0 & 0 \\
\hline $300-600$ & 0 & 0 & 0 & 0 & 0 & 0 & 0 & 0 & 0 & 0 & 0 \\
\hline $600-1200$ & 0 & 0 & 0 & 0 & 0 & 0 & 0 & 0 & 0 & 0 & 0 \\
\hline $\begin{array}{l}\text { BANDAS DE } \\
\text { FRECUENCIA }\end{array}$ & \multicolumn{11}{|c|}{ POTENCIAS DE RUIDO CARDIACO ESTIMADO SIN TW } \\
\hline 0-300 & 0 & 0 & 0 & 0 & 0 & 0 & 0 & 0 & 0 & 0 & 0 \\
\hline $300-600$ & 0 & 0 & 0 & 0 & 0 & 0 & 0 & 0 & 0 & 0 & 0 \\
\hline $600-1200$ & 0 & 0 & 0 & 0 & 0 & 0 & 0 & 0 & 0 & 0 & 0 \\
\hline $\begin{array}{l}\text { BANDAS DE } \\
\text { FRECUENCIA }\end{array}$ & \multicolumn{11}{|c|}{ POTENCIAS DE RUIDO CARDIACO ESTIMADO CON TW } \\
\hline $0-300$ & 0 & 0 & 0 & 0 & 0 & 0 & 0 & 0 & 0 & 0 & 0 \\
\hline $300-600$ & 0 & 0 & 0 & 0 & 0 & 0 & 0 & 0 & 0 & 0 & 0 \\
\hline $600-1200$ & 0 & 0 & 0 & 0 & 0 & 0 & 0 & 0 & 0 & 0 & \\
\hline
\end{tabular}

Tabla 5. 5.f. Potencias calculadas de la señal en el dominio original obtenida de la línea axilar derecha basal para un flujo de $1.5 \mathrm{~L} / \mathrm{seg}$. 


\begin{tabular}{|c|c|c|c|c|c|c|c|c|c|c|c|}
\hline \multirow{4}{*}{ INTERVALOS } & \multicolumn{11}{|c|}{ MIT } \\
\hline & \multicolumn{4}{|c|}{ APNEA } & \multicolumn{7}{|c|}{ ZONA DE RESPIRACIÓN } \\
\hline & 208 & 4135 & 8041 & 11626 & 18775 & 27584 & 30359 & 32822 & 35472 & 38685 & 41572 \\
\hline & 2078 & 6005 & 9911 & 13496 & 19578 & 28374 & 31149 & 34692 & 37342 & 39475 & 42362 \\
\hline $\begin{array}{l}\text { BANDAS DE } \\
\text { FRECUENCIA }\end{array}$ & \multicolumn{11}{|c|}{ SEÑAL ORIGINAL } \\
\hline $0-300$ & 824.68 & 836.13 & 851.92 & 866.2 & 649.56 & 662.01 & 798.76 & 1338.1 & 1234.4 & 682.74 & 811.63 \\
\hline $300-600$ & 107.4 & 114.77 & 115.97 & 137.56 & 56.927 & 48.233 & 62.718 & 111.78 & 120.42 & 53.32 & 77.809 \\
\hline $600-1200$ & 272.2 & 262.38 & 273.51 & 275.16 & 129.87 & 125.71 & 128.02 & 278.35 & 294.46 & 127.79 & 130.85 \\
\hline $\begin{array}{l}\text { BANDAS DE } \\
\text { FRECUENCIA }\end{array}$ & \multicolumn{11}{|c|}{ POTENCIAS DE LAS SEÑALES DE SONIDO RESPIRATORIO ESTIMADO SIN TW } \\
\hline $0-300$ & 551.86 & 502.06 & 495.09 & 548.44 & 791.03 & 358.45 & 608.44 & 1931.7 & 1544.1 & 430.19 & 748.53 \\
\hline $300-600$ & 61.675 & 62.802 & 57.646 & 59.802 & 34.111 & 32.198 & 26.888 & 55.717 & 57.336 & 32.047 & 29.251 \\
\hline $600-1200$ & 14.531 & 12.118 & 11.763 & 10.179 & 5.9811 & 4.3728 & 5.8752 & 10.062 & 7.4284 & 3.8931 & 10.235 \\
\hline $\begin{array}{l}\text { BANDAS DE } \\
\text { FRECUENCIA }\end{array}$ & \multicolumn{11}{|c|}{ POTENCIAS DE LAS SEÑALES DE SONIDO RESPIRATORIO ESTIMADO CON TW } \\
\hline $0-300$ & 547.15 & 490.58 & 500.82 & 499.5 & 796.1 & 358.45 & 620.76 & 1927.5 & 1544.1 & 430.19 & 750.24 \\
\hline $300-600$ & 60.231 & 61.394 & 70.125 & 61.757 & 34.092 & 32.198 & 28.089 & 72.552 & 57.336 & 32.047 & 58.66 \\
\hline $600-1200$ & 12.552 & 11.079 & 20.649 & 11.516 & 6.3166 & 4.3728 & 8.8671 & 22.988 & 7.4284 & 3.8931 & 44.302 \\
\hline $\begin{array}{l}\text { BANDAS DE } \\
\text { FRECUENCIA }\end{array}$ & \multicolumn{11}{|c|}{ POTENCIAS DE RUIDO CARDIACO ESTIMADO SIN TW } \\
\hline 0-300 & 582.55 & 581.76 & 560.55 & 615.27 & 482.51 & 359.42 & 355.65 & 817.92 & 772.38 & 441.77 & 379.26 \\
\hline $300-600$ & 4.8057 & 5.3184 & 13.703 & 13.144 & 13.683 & 5.9938 & 6.5644 & 19.715 & 19.845 & 18.179 & 11.219 \\
\hline $600-1200$ & 3.4156 & 1.8893 & 7.6831 & 6.5486 & 5.4132 & 3.632 & 4.2363 & 7.2966 & 11.398 & 5.9388 & 4.7306 \\
\hline $\begin{array}{l}\text { BANDAS DE } \\
\text { FRECUENCIA }\end{array}$ & \multicolumn{11}{|c|}{ POTENCIAS DE RUIDO CARDIACO ESTIMADO CON TW } \\
\hline $0-300$ & 589.58 & 565.9 & 593.96 & 625.77 & 447.34 & 366.45 & 357.63 & 771.85 & 750.13 & 460.6 & 396.03 \\
\hline $300-600$ & 29.292 & 6.5362 & 27.045 & 43.896 & 18.699 & 14.26 & 10.176 & 36.778 & 80.44 & 28.812 & 42.085 \\
\hline $600-1200$ & 20.179 & 2.4907 & 16.158 & 23.47 & 6.6764 & 6.7195 & 6.9402 & 21.892 & 57.585 & 11.22 & 22.73 \\
\hline
\end{tabular}

Tabla 5. 5.g. Potencias calculadas de la señal en el dominio original obtenida de la zona de auscultación de la válvula mitral para un flujo de $1.5 \mathrm{~L} / \mathrm{seg}$.

\begin{tabular}{|c|c|c|c|c|c|c|c|c|c|c|c|}
\hline \multirow{4}{*}{ INTERVALOS } & \multicolumn{11}{|c|}{ TRI } \\
\hline & \multicolumn{4}{|c|}{ APNEA } & \multicolumn{7}{|c|}{ ZONA DE RESPIRACIÓN } \\
\hline & 430 & 4365 & 8204 & 11691 & 18580 & 27545 & 30320 & 32897 & 35502 & 38571 & 41009 \\
\hline & 1172 & 5150 & 8918 & 12862 & 19751 & 28330 & 31491 & 34068 & 37026 & 39280 & 42226 \\
\hline \multicolumn{12}{|l|}{$\begin{array}{l}\text { BANDAS DE } \\
\text { FRECUENCIA }\end{array}$} \\
\hline $0-300$ & 1625.8 & 1598.2 & 1402.8 & 1838.3 & 2602.9 & 1743 & 2264.1 & 2466.6 & 2433.7 & 1765.5 & 2085.5 \\
\hline $300-600$ & 47.024 & 41.204 & 48.972 & 87.923 & 93.622 & 58.454 & 115.95 & 80.254 & 94.782 & 52.427 & 86.199 \\
\hline $600-1200$ & 121.54 & 113.26 & 114.84 & 161.44 & 181.63 & 111.04 & 191.24 & 153.22 & 190.78 & 106.92 & 175.37 \\
\hline $\begin{array}{l}\text { BANDAS DE } \\
\text { FRECUENCIA }\end{array}$ & \multicolumn{11}{|c|}{ POTENCIAS DE LAS SEÑALES DE SONIDO RESPIRATORIO ESTIMADO SIN TW } \\
\hline $0-300$ & 770.12 & 1299.8 & 928.12 & 896.39 & 4049.9 & 953.16 & 2511.9 & 3490.1 & 2734.4 & 1237.2 & 1613 \\
\hline $300-600$ & 9.6348 & 2.8199 & 6.4928 & 5.3243 & 10.031 & 5.1411 & 8.4542 & 8.189 & 12.888 & 11.397 & 9.5877 \\
\hline $600-1200$ & 0.8859 & 0.33615 & 0.67809 & 0.5684 & 1.3767 & 0.6422 & 1.0656 & 0.93586 & 1.5628 & 1.3749 & 1.0733 \\
\hline $\begin{array}{l}\text { BANDAS DE } \\
\text { FRECUENCIA }\end{array}$ & \multicolumn{11}{|c|}{ POTENCIAS DE LAS SEÑALES DE SONIDO RESPIRATORIO ESTIMADO CON TW } \\
\hline 0-300 & 770.12 & 1299.8 & 928.12 & 896.39 & 4049.9 & 953.16 & 2511.9 & 3490.1 & 2734.4 & 1237.2 & 1613 \\
\hline $300-600$ & 9.6348 & 2.8199 & 6.4928 & 5.3243 & 10.031 & 5.1411 & 8.4542 & 8.189 & 12.888 & 11.397 & 9.5877 \\
\hline $600-1200$ & 0.8859 & 0.33615 & 0.67809 & 0.5684 & 1.3767 & 0.6422 & 1.0656 & 0.93586 & 1.5628 & 1.3749 & 1.0733 \\
\hline $\begin{array}{l}\text { BANDAS DE } \\
\text { FRECUENCIA }\end{array}$ & \multicolumn{11}{|c|}{ POTENCIAS DE RUIDO CARDIACO ESTIMADO SIN TW } \\
\hline $0-300$ & 1004.2 & 1095.3 & 844.88 & 1264.6 & 1070.6 & 1216.5 & 1468.8 & 2360.4 & 1751.8 & 1390.6 & 1640.5 \\
\hline $300-600$ & 11.402 & 20.433 & 15.928 & 30.883 & 22.649 & 51.376 & 57.115 & 130.42 & 97.426 & 42.073 & 57.357 \\
\hline $600-1200$ & 1.0594 & 2.7155 & 1.8933 & 8.0143 & 10.911 & 30.627 & 31.279 & 53.751 & 43.472 & 13.78 & 19.325 \\
\hline $\begin{array}{l}\text { BANDAS DE } \\
\text { FRECUENCIA }\end{array}$ & \multicolumn{11}{|c|}{ POTENCIAS DE RUIDO CARDIACO ESTIMADO CON TW } \\
\hline $0-300$ & 964.47 & 1130.3 & 921.07 & 1300.4 & 1140.1 & 1294.6 & 1635 & 2416.6 & 1798.1 & 1391.6 & 1707.2 \\
\hline $300-600$ & 16.443 & 23.366 & 70.775 & 54.615 & 84.161 & 190.35 & 177.15 & 328.07 & 243.08 & 46.417 & 149.53 \\
\hline $600-1200$ & 4.1761 & 15.435 & 14.956 & 19.218 & 34.317 & 96.525 & 112.07 & 130.31 & 77.914 & 23.232 & 57.669 \\
\hline
\end{tabular}

Tabla 5. 5.h. Potencias calculadas de la señal en el dominio original obtenida de la zona de auscultación de la válvula tricúspide para un flujo de $1.5 \mathrm{~L} / \mathrm{seg}$. 


\begin{tabular}{|c|c|c|c|c|c|c|c|c|c|c|c|}
\hline \multirow{4}{*}{ INTERVALOS } & \multicolumn{11}{|c|}{ PUL } \\
\hline & \multicolumn{4}{|c|}{ APNEA } & \multicolumn{7}{|c|}{ ZONA DE RESPIRACIÓN } \\
\hline & 301 & 4303 & 8123 & 11780 & 18770 & 0 & 30535 & 33599 & 0 & 38547 & 0 \\
\hline & 1407 & 6426 & 10246 & 13903 & 21432 & 0 & 31641 & 35722 & 0 & 40670 & 0 \\
\hline \multicolumn{12}{|l|}{$\begin{array}{l}\text { BANDAS DE } \\
\text { FRECUENCIA }\end{array}$} \\
\hline 0-300 & 5254 & 9121.6 & 9670 & 9485.5 & 21951 & 0 & 23981 & 22653 & 0 & 11162 & 0 \\
\hline $300-600$ & 75.698 & 168.75 & 180.93 & 232.43 & 708.62 & 0 & 310.55 & 206.68 & 0 & 242.56 & 0 \\
\hline $600-1200$ & 159.03 & 319.62 & 331.74 & 318.19 & 486.31 & 0 & 206.54 & 328.59 & 0 & 356.11 & 0 \\
\hline $\begin{array}{l}\text { BANDAS DE } \\
\text { FRECUENCIA }\end{array}$ & \multicolumn{11}{|c|}{ POTENCIAS DE LAS SEÑALES DE SONIDO RESPIRATORIO ESTIMADO SIN TW } \\
\hline $0-300$ & 187.58 & 512.99 & 673.79 & 687.65 & 1603.9 & 0 & 667.04 & 990.06 & 0 & 1039 & 0 \\
\hline $300-600$ & 5.5575 & 9.7691 & 15.162 & 12.954 & 19.273 & 0 & 10.12 & 20.575 & 0 & 22.84 & 0 \\
\hline $600-1200$ & 1.0078 & 2.6846 & 7.2721 & 5.2802 & 4.886 & 0 & 3.1355 & 7.4258 & 0 & 7.9316 & 0 \\
\hline $\begin{array}{l}\text { BANDAS DE } \\
\text { FRECUENCIA }\end{array}$ & \multicolumn{11}{|c|}{ POTENCIAS DE LAS SEÑALES DE SONIDO RESPIRATORIO ESTIMADO CON TW } \\
\hline $0-300$ & 186.21 & 550.86 & 675.63 & 660.4 & 1559.4 & 0 & 711.36 & 966.9 & 0 & 981.67 & 0 \\
\hline $300-600$ & 23.895 & 10.496 & 27.876 & 43.824 & 24.867 & 0 & 13.37 & 37.566 & 0 & 33.667 & 0 \\
\hline $600-1200$ & 14.841 & 3.0415 & 15.558 & 21.969 & 6.7723 & 0 & 5.5733 & 21.295 & 0 & 13.311 & 0 \\
\hline $\begin{array}{l}\text { BANDAS DE } \\
\text { FRECUENCIA }\end{array}$ & \multicolumn{11}{|c|}{ POTENCIAS DE RUIDO CARDIACO ESTIMADO SIN TW } \\
\hline $0-300$ & 1843.4 & 7623.2 & 8134.9 & 8451.1 & 12910 & 0 & 3229.6 & 14155 & 0 & 8583.8 & 0 \\
\hline $300-600$ & 4.6159 & 104.88 & 115.68 & 163.96 & 460.27 & 0 & 63.413 & 148.78 & 0 & 257.62 & 0 \\
\hline $600-1200$ & 0.62552 & 30.312 & 45.837 & 73.984 & 287.53 & 0 & 47.244 & 83.241 & 0 & 129.53 & 0 \\
\hline $\begin{array}{l}\text { BANDAS DE } \\
\text { FRECUENCIA }\end{array}$ & \multicolumn{11}{|c|}{ POTENCIAS DE RUIDO CARDIACO ESTIMADO CON TW } \\
\hline $0-300$ & 1844.5 & 7619 & 8328.7 & 8554 & 12925 & 0 & 3281.5 & 14982 & 0 & 8567.7 & 0 \\
\hline $300-600$ & 4.555 & 103.17 & 149.62 & 164.66 & 518.07 & 0 & 136.43 & 314.94 & 0 & 231.08 & 0 \\
\hline $600-1200$ & 0.58905 & 28.869 & 59.04 & 67.902 & 344.54 & 0 & 100.97 & 154.19 & 0 & 211.68 & 0 \\
\hline
\end{tabular}

Tabla 5. 5.i. Potencias calculadas de la señal en el dominio original obtenida de la zona de auscultación de la válvula pulmonar para un flujo de $1.5 \mathrm{~L} / \mathrm{seg}$.

\begin{tabular}{|c|c|c|c|c|c|c|c|c|c|c|c|}
\hline \multirow{4}{*}{ INTERVALOS } & \multicolumn{11}{|c|}{ AOR } \\
\hline & \multicolumn{4}{|c|}{ APNEA } & \multicolumn{7}{|c|}{ ZONA DE RESPIRACIÓN } \\
\hline & 288 & 3863 & 8121 & 10995 & 18984 & 26654 & 30295 & 33027 & 35301 & 38497 & 40789 \\
\hline & 1303 & 5769 & 9136 & 12901 & 19782 & 28560 & 31310 & 34042 & 37207 & 39512 & 42695 \\
\hline BANDAS DE & \multicolumn{11}{|c|}{ SEÑAL ORIGINAL } \\
\hline $0-300$ & 1803 & 2685.9 & 1579.2 & 1874.3 & 2196.7 & 2876.1 & 2750.9 & 3032 & 4101.1 & 2262.3 & 4815.8 \\
\hline $300-600$ & 52.024 & 106 & 60.356 & 100.48 & 134.84 & 121.56 & 340.77 & 77.68 & 133.88 & 127.3 & 564.22 \\
\hline $600-1200$ & 138.91 & 252.85 & 133.82 & 236.06 & 122.66 & 242.25 & 201.48 & 137.58 & 254.76 & 143.86 & 343 \\
\hline $\begin{array}{l}\text { BANDAS DE } \\
\text { FRECUENCIA }\end{array}$ & \multicolumn{11}{|c|}{ POTENCIAS DE LAS SEÑALES DE SONIDO RESPIRATORIO ESTIMADO SIN TW } \\
\hline $0-300$ & 1336.7 & 2012.4 & 1128.7 & 1197.7 & 1588.1 & 2088.7 & 1228.8 & 2469.7 & 2520.7 & 874.82 & 1925.3 \\
\hline $300-600$ & 35.078 & 64.065 & 29.484 & 61.886 & 31.089 & 91.374 & 60.682 & 132.46 & 138.05 & 64.653 & 86.995 \\
\hline $600-1200$ & 5.4807 & 10.002 & 5.485 & 12.127 & 9.8575 & 46.383 & 24.047 & 52.443 & 55.6 & 18.758 & 24.327 \\
\hline $\begin{array}{l}\text { BANDAS DE } \\
\text { FRECUENCIA }\end{array}$ & \multicolumn{11}{|c|}{ POTENCIAS DE LAS SEÑALES DE SONIDO RESPIRATORIO ESTIMADO CON TW } \\
\hline $0-300$ & 1321 & 1959.9 & 1147.9 & 1227.9 & 1796.4 & 2214.8 & 1430.4 & 2422.9 & 2720.4 & 858.15 & 2070.7 \\
\hline $300-600$ & 39.133 & 69.077 & 84.083 & 82.84 & 70.537 & 228.89 & 177.58 & 325.93 & 281.69 & 65.869 & 180.1 \\
\hline $600-1200$ & 7.7648 & 22.352 & 18.257 & 23.28 & 23.626 & 112.01 & 105.17 & 129.17 & 88.662 & 27.627 & 61.988 \\
\hline $\begin{array}{l}\text { BANDAS DE } \\
\text { FRECUENCIA }\end{array}$ & \multicolumn{11}{|c|}{ POTENCIAS DE RUIDO CARDIACO ESTIMADO SIN TW } \\
\hline 0-300 & 1043.7 & 1703.3 & 1025 & 1223.3 & 1492.4 & 2481.7 & 969 & 1355.1 & 1980.5 & 1195.9 & 3591.2 \\
\hline $300-600$ & 23.357 & 19.975 & 43.485 & 19.958 & 54.361 & 85.788 & 36.333 & 38.314 & 33.014 & 31.947 & 70.654 \\
\hline $600-1200$ & 4.4225 & 8.6256 & 8.1215 & 7.8167 & 18.255 & 72.704 & 23.442 & 19.102 & 19.453 & 15.388 & 34.404 \\
\hline $\begin{array}{l}\text { BANDAS DE } \\
\text { FRECUENCIA }\end{array}$ & \multicolumn{11}{|c|}{ POTENCIAS DE RUIDO CARDIACO ESTIMADO CON TW } \\
\hline $0-300$ & 1070.7 & 1697.7 & 1038.8 & 1267.7 & 1593.4 & 2458.7 & 976.08 & 1304.6 & 1967 & 1228.5 & 3563.5 \\
\hline $300-600$ & 32.403 & 37.97 & 57.061 & 20.964 & 225.24 & 150.2 & 87.992 & 40.365 & 40.47 & 93.514 & 217.85 \\
\hline $600-1200$ & 9.1391 & 19.108 & 12.733 & 9.6339 & 141.68 & 104.01 & 42.995 & 32.503 & 33.097 & 131.04 & 80.401 \\
\hline
\end{tabular}

Tabla 5. 5.j. $\quad$ Potencias calculadas de la señal en el dominio original obtenida de la zona de auscultación de la válvula aórtica para un flujo de $1.5 \mathrm{~L} / \mathrm{seg}$. 


\begin{tabular}{|c|c|c|c|c|c|c|c|c|c|c|c|}
\hline \multirow{4}{*}{ INTERVALOS } & \multicolumn{11}{|c|}{ PRE } \\
\hline & \multicolumn{4}{|c|}{ APNEA } & \multicolumn{7}{|c|}{ ZONA DE RESPIRACIÓN } \\
\hline & 403 & 4319 & 8166 & 11826 & 18815 & 27470 & 30429 & 33125 & 35809 & 38717 & 41288 \\
\hline & 1155 & 5204 & 9096 & 12658 & 19751 & 28545 & 31261 & 33878 & 36817 & 39549 & 42473 \\
\hline $\begin{array}{l}\text { BANDAS DE } \\
\text { FRECUENCIA }\end{array}$ & \multicolumn{11}{|c|}{ SEÑAL ORIGINAL } \\
\hline $0-300$ & 533.88 & 626.04 & 606.26 & 594.27 & 762.46 & 988.02 & 1052.5 & 667.8 & 896.11 & 812.78 & 1506.8 \\
\hline $300-600$ & 40.813 & 53.43 & 54.018 & 63.459 & 61.908 & 66.254 & 117.34 & 49.378 & 60.141 & 60.503 & 210.31 \\
\hline $600-1200$ & 102.73 & 135.72 & 138.03 & 153.83 & 155.03 & 164.54 & 167.68 & 118.44 & 166.58 & 135.34 & 260.04 \\
\hline $\begin{array}{l}\text { BANDAS DE } \\
\text { FRECUENCIA }\end{array}$ & \multicolumn{11}{|c|}{ POTENCIAS DE LAS SEÑALES DE SONIDO RESPIRATORIO ESTIMADO SIN TW } \\
\hline $0-300$ & 4003.5 & 5352.6 & 4798.5 & 4283.7 & 9624.8 & 3953.4 & 23665 & 6153.5 & 6284.3 & 4749.3 & 13084 \\
\hline $300-600$ & 18.184 & 58.116 & 63.682 & 61.188 & 340.2 & 47.838 & 65.853 & 87.649 & 82.81 & 58.861 & 225.19 \\
\hline $600-1200$ & 2.2666 & 6.9069 & 7.3436 & 7.5901 & 201.54 & 6.9002 & 23.52 & 79.86 & 38.092 & 9.2154 & 141.13 \\
\hline $\begin{array}{l}\text { BANDAS DE } \\
\text { FRECUENCIA }\end{array}$ & \multicolumn{11}{|c|}{ POTENCIAS DE LAS SEÑALES DE SONIDO RESPIRATORIO ESTIMADO CON TW } \\
\hline $0-300$ & 4002.5 & 5337 & 4995 & 4274.2 & 9683.1 & 3953.4 & 23683 & 6134.6 & 6276.4 & 4729.7 & 12873 \\
\hline $300-600$ & 18.066 & 55.321 & 84.903 & 72.261 & 356.34 & 47.838 & 128.94 & 95.534 & 69.927 & 65.599 & 224.6 \\
\hline $600-1200$ & 2.2577 & 5.9273 & 22.694 & 16.505 & 226.7 & 6.9002 & 73.814 & 82.189 & 60.783 & 21.425 & 135.03 \\
\hline $\begin{array}{l}\text { BANDAS DE } \\
\text { FRECUENCIA }\end{array}$ & \multicolumn{11}{|c|}{ POTENCIAS DE RUIDO CARDIACO ESTIMADO SIN TW } \\
\hline $0-300$ & 314.95 & 324.72 & 402.92 & 406.67 & 460.6 & 664.85 & 641.21 & 521.45 & 866.68 & 579.86 & 972.59 \\
\hline $300-600$ & 2.7709 & 6.2467 & 8.7537 & 5.5878 & 9.6706 & 28.896 & 31.52 & 26.139 & 34.443 & 35.95 & 43.757 \\
\hline $600-1200$ & 0.75012 & 1.0636 & 1.9922 & 1.6844 & 3.4703 & 30.421 & 21.025 & 11.379 & 10.127 & 32.033 & 33.217 \\
\hline $\begin{array}{l}\text { BANDAS DE } \\
\text { FRECUENCIA }\end{array}$ & \multicolumn{11}{|c|}{ POTENCIAS DE RUIDO CARDIACO ESTIMADO CON TW } \\
\hline $0-300$ & 317.05 & 346.23 & 414.91 & 402.53 & 440.37 & 713.95 & 752.8 & 508.67 & 891.62 & 648.87 & 949.64 \\
\hline $300-600$ & 10.006 & 23.042 & 14.252 & 8.0934 & 19.735 & 86.4 & 91.348 & 81.94 & 78.91 & 100.55 & 105.05 \\
\hline $600-1200$ & 2.232 & 6.3634 & 5.6813 & 3.1438 & 11.967 & 77.597 & 61.356 & 36.218 & 29.042 & 57.102 & 88.318 \\
\hline
\end{tabular}

Tabla 5. 5.k. Potencias calculadas de la señal en el dominio original obtenida de la zona de auscultación precordial promedio para un flujo de $1.5 \mathrm{~L} / \mathrm{seg}$. 
Tabla 5. 6 Potencias calculadas de la señal en el dominio original obtenidas para un flujo de 2.0L/seg

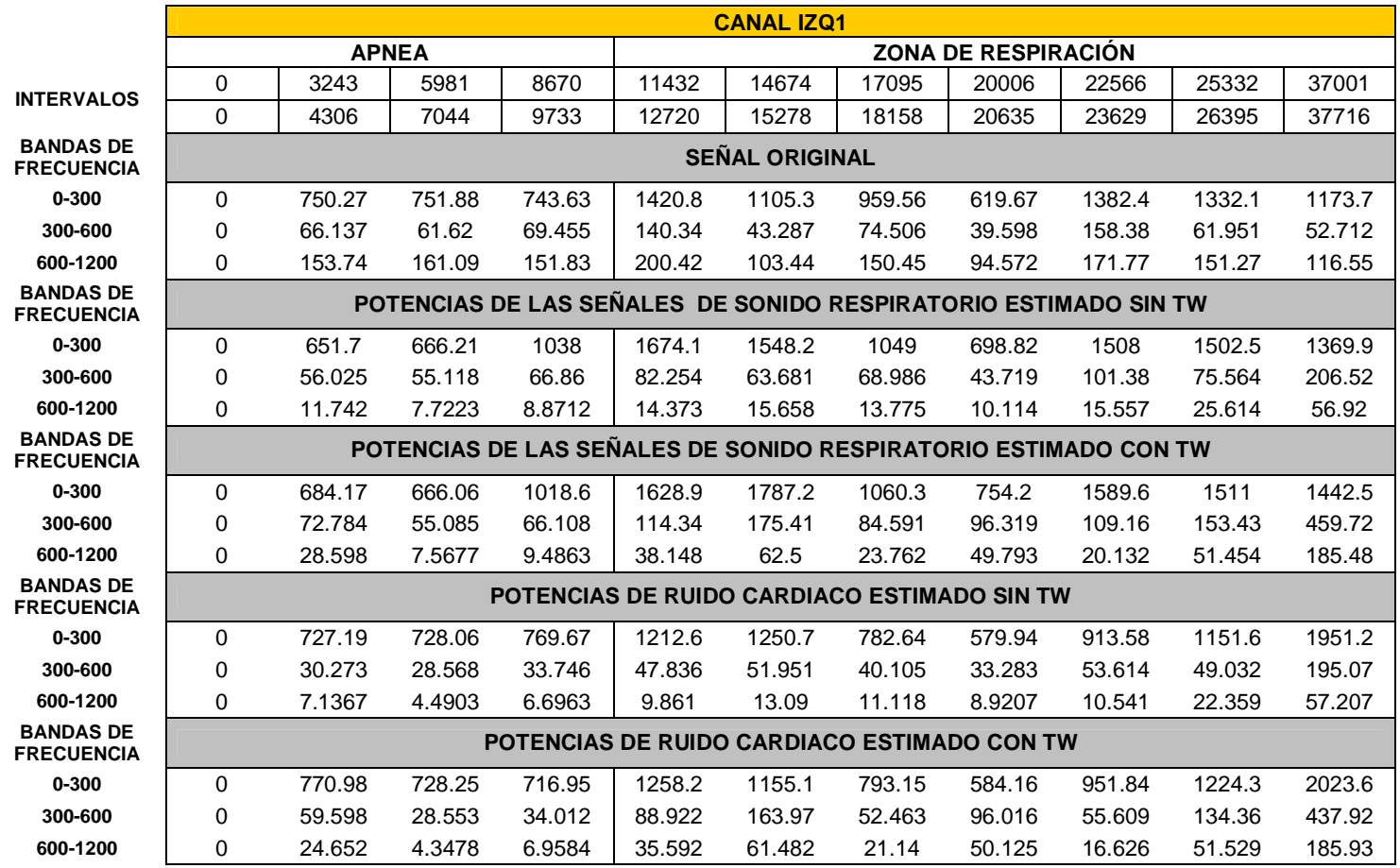

Tabla 5. 6.a. Potencias calculadas de la señal en el dominio original obtenida de la línea axilar izquierda apical para un flujo de $2.0 \mathrm{~L} / \mathrm{seg}$.

\begin{tabular}{|c|c|c|c|c|c|c|c|c|c|c|c|}
\hline \multirow{4}{*}{ INTERVALOS } & \multicolumn{11}{|c|}{ CANAL IZQ2 } \\
\hline & \multicolumn{4}{|c|}{ APNEA } & \multicolumn{7}{|c|}{ ZONA DE RESPIRACIÓN } \\
\hline & 514 & 3129 & 6003 & 8878 & 11701 & 14305 & 16913 & 19730 & 22391 & 25223 & 36585 \\
\hline & 1933 & 4625 & 7409 & 10284 & 13107 & 15770 & 18319 & 21136 & 23932 & 26430 & 37991 \\
\hline $\begin{array}{l}\text { BANDAS DE } \\
\text { FRECUENCIA }\end{array}$ & \multicolumn{11}{|c|}{ SEÑAL ORIGINAL } \\
\hline $0-300$ & 2889.8 & 2691.8 & 2779.2 & 2901 & 5513.1 & 8225.1 & 3082.8 & 2337.2 & 5914.6 & 5277.1 & 8408.1 \\
\hline $300-600$ & 120.48 & 101.63 & 86.626 & 85.937 & 216.35 & 99.174 & 82.496 & 85.348 & 232.1 & 86.639 & 80.34 \\
\hline $600-1200$ & 185.7 & 192.85 & 198.44 & 189.42 & 180.94 & 195.58 & 173.62 & 187.01 & 200.68 & 155.64 & 180.35 \\
\hline $\begin{array}{l}\text { BANDAS DE } \\
\text { FRECUENCIA }\end{array}$ & \multicolumn{11}{|c|}{ POTENCIAS DE LAS SEÑALES DE SONIDO RESPIRATORIO ESTIMADO SIN TW } \\
\hline $0-300$ & 1350.1 & 937.17 & 1383.2 & 1843.6 & 4218.2 & 5940.5 & 2334.7 & 1809.7 & 4466.6 & 3781.8 & 5749.9 \\
\hline $300-600$ & 117.57 & 94.84 & 82.045 & 102.35 & 182.45 & 135.58 & 176.16 & 150.14 & 199.07 & 114.85 & 144.41 \\
\hline $600-1200$ & 44.952 & 41.978 & 41.694 & 55.846 & 75.061 & 67.656 & 122.25 & 99.287 & 86.647 & 39.565 & 79.893 \\
\hline $\begin{array}{l}\text { BANDAS DE } \\
\text { FRECUENCIA }\end{array}$ & \multicolumn{11}{|c|}{ POTENCIAS DE LAS SEÑALES DE SONIDO RESPIRATORIO ESTIMADO CON TW } \\
\hline $0-300$ & 1416.5 & 927.35 & 1351.2 & 1436.9 & 4117 & 5979.1 & 2333.3 & 1959.6 & 4488.9 & 3623.3 & 6374.8 \\
\hline $300-600$ & 123.84 & 94.986 & 93.136 & 88.913 & 177.62 & 183.16 & 328.08 & 250.12 & 255.22 & 142.29 & 246.7 \\
\hline $600-1200$ & 51.334 & 43.982 & 46.187 & 50.594 & 71.392 & 84.822 & 167.48 & 126.57 & 123.72 & 52.014 & 137.07 \\
\hline $\begin{array}{l}\text { BANDAS DE } \\
\text { FRECUENCIA }\end{array}$ & \multicolumn{11}{|c|}{ POTENCIAS DE RUIDO CARDIACO ESTIMADO SIN TW } \\
\hline $0-300$ & 2215.9 & 2259.6 & 2279.5 & 2652.2 & 3085.3 & 5704.7 & 1682.3 & 1692.5 & 3361 & 3239.9 & 5299.3 \\
\hline $300-600$ & 23.818 & 14.342 & 21.553 & 54.02 & 66.275 & 92.33 & 129.53 & 108.08 & 94.9 & 70.737 & 112.37 \\
\hline $600-1200$ & 9.0471 & 4.8025 & 5.156 & 22.027 & 33.374 & 41.338 & 102.65 & 69.179 & 55.042 & 12.623 & 48.074 \\
\hline $\begin{array}{l}\text { BANDAS DE } \\
\text { FRECUENCIA }\end{array}$ & \multicolumn{11}{|c|}{ POTENCIAS DE RUIDO CARDIACO ESTIMADO CON TW } \\
\hline $0-300$ & 2249.9 & 2236.6 & 2312.6 & 2409.4 & 2954.5 & 5859.3 & 1730.1 & 1819.7 & 3258.2 & 3292.5 & 5527.1 \\
\hline $300-600$ & 29.72 & 18.317 & 39.921 & 24.515 & 52.801 & 144.19 & 291.49 & 224.69 & 197.44 & 115.16 & 223.97 \\
\hline $600-1200$ & 18.274 & 8.7853 & 13.342 & 16.492 & 34.376 & 66.445 & 156.39 & 107.8 & 102.03 & 31.879 & 114.7 \\
\hline
\end{tabular}

Tabla 5. 6.b. Potencias calculadas de la señal en el dominio original obtenida de la línea axilar izquierda media para un flujo de $2.0 \mathrm{~L} / \mathrm{seg}$. 


\begin{tabular}{|c|c|c|c|c|c|c|c|c|c|c|c|}
\hline \multirow{4}{*}{ INTERVALOS } & \multicolumn{11}{|c|}{ CANAL IZQ3 } \\
\hline & \multicolumn{4}{|c|}{ APNEA } & \multicolumn{7}{|c|}{ ZONA DE RESPIRACIÓN } \\
\hline & 625 & 3316 & 6160 & 8962 & 11810 & 14588 & 17181 & 20042 & 22438 & 25265 & 36932 \\
\hline & 1748 & 4439 & 7283 & 10128 & 12933 & 15711 & 18304 & 21173 & 23597 & 26388 & 37763 \\
\hline $\begin{array}{l}\text { BANDAS DE } \\
\text { FRECUENCIA }\end{array}$ & \multicolumn{11}{|c|}{ SEÑAL ORIGINAL } \\
\hline $0-300$ & 967.06 & 1007.3 & 1055.9 & 1086 & 1907.3 & 1825.8 & 1380.4 & 1048.6 & 1849.4 & 2702.5 & 2013.9 \\
\hline $300-600$ & 97.23 & 92.873 & 68.553 & 68.828 & 131.92 & 75.708 & 64.776 & 68.102 & 127.59 & 81.551 & 51.062 \\
\hline $600-1200$ & 177.92 & 187.52 & 180.99 & 179.97 & 197.27 & 167.9 & 169.66 & 176.92 & 201.93 & 177.32 & 124.17 \\
\hline $\begin{array}{l}\text { BANDAS DE } \\
\text { FRECUENCIA }\end{array}$ & \multicolumn{11}{|c|}{ POTENCIAS DE LAS SEÑALES DE SONIDO RESPIRATORIO ESTIMADO SIN TW } \\
\hline $0-300$ & 526.35 & 507.9 & 319.68 & 374.49 & 1363.2 & 1401.1 & 806.95 & 921.37 & 2239.3 & 2453.3 & 1268.6 \\
\hline $300-600$ & 48.283 & 40.27 & 31.909 & 40.321 & 164.28 & 143.81 & 41.238 & 68.399 & 300.18 & 222.13 & 65.639 \\
\hline $600-1200$ & 6.343 & 7.3569 & 6.1415 & 7.9438 & 95.156 & 80.675 & 12.683 & 17.92 & 237.34 & 171.04 & 65.579 \\
\hline $\begin{array}{c}\text { BANDAS DE } \\
\text { FRECUENCIA }\end{array}$ & \multicolumn{11}{|c|}{ POTENCIAS DE LAS SEÑALES DE SONIDO RESPIRATORIO ESTIMADO CON TW } \\
\hline $0-300$ & 481.5 & 527.38 & 321.46 & 351.99 & 1437.6 & 1860 & 758.93 & 986.82 & 2805.1 & 2823.1 & 1379.4 \\
\hline $300-600$ & 52.099 & 54.163 & 32.086 & 42.17 & 512.22 & 454.73 & 86.822 & 212.74 & 1108.1 & 850.64 & 189.61 \\
\hline $600-1200$ & 15.69 & 21.544 & 5.9534 & 12.059 & 503.9 & 370.39 & 37.364 & 111.7 & 1174.1 & 1089.6 & 289.31 \\
\hline $\begin{array}{l}\text { BANDAS DE } \\
\text { FRECUENCIA }\end{array}$ & \multicolumn{11}{|c|}{ POTENCIAS DE RUIDO CARDIACO ESTIMADO SIN TW } \\
\hline $0-300$ & 746.81 & 741.72 & 842.08 & 1031.7 & 1516.1 & 1665 & 645.04 & 1041.4 & 2137.7 & 2517.7 & 1538.9 \\
\hline $300-600$ & 12.645 & 14.236 & 4.2916 & 14.067 & 142.7 & 129.27 & 19.907 & 54.753 & 285.73 & 215.16 & 58.484 \\
\hline $600-1200$ & 2.5607 & 4.4487 & 1.5985 & 4.601 & 93.395 & 80.143 & 9.4934 & 16.903 & 236.57 & 170.92 & 63.635 \\
\hline $\begin{array}{l}\text { BANDAS DE } \\
\text { FRECUENCIA }\end{array}$ & \multicolumn{11}{|c|}{ POTENCIAS DE RUIDO CARDIACO ESTIMADO CON TW } \\
\hline $0-300$ & 774.55 & 786.66 & 844.47 & 1017.5 & 1723.4 & 2132.6 & 616.03 & 1147.2 & 2737.6 & 2772.2 & 1524.5 \\
\hline $300-600$ & 28.97 & 33.448 & 4.6372 & 20.517 & 503.48 & 447.09 & 78.251 & 207.73 & 1100.5 & 848.2 & 183.36 \\
\hline $600-1200$ & 12.697 & 18.998 & 1.418 & 9.095 & 502.56 & 370.35 & 36.727 & 111.34 & 1174.1 & 1089.6 & 288.98 \\
\hline
\end{tabular}

Tabla 5. 6.c. Potencias calculadas de la señal en el dominio original obtenida de la línea axilar izquierda basal para un flujo de $2.0 \mathrm{~L} / \mathrm{seg}$.

\begin{tabular}{|c|c|c|c|c|c|c|c|c|c|c|c|}
\hline \multirow{4}{*}{ INTERVALOS } & \multicolumn{11}{|c|}{ DER1 } \\
\hline & \multicolumn{4}{|c|}{ APNEA } & \multicolumn{7}{|c|}{ ZONA DE RESPIRACIÓN } \\
\hline & 729 & 3462 & 6225 & 0 & 12053 & 14706 & 17308 & 20148 & 23020 & 25581 & 0 \\
\hline & 1367 & 4100 & 6863 & 0 & 12691 & 15344 & 17946 & 20792 & 24051 & 26361 & 0 \\
\hline $\begin{array}{l}\text { BANDAS DE } \\
\text { FRECUENCIA }\end{array}$ & \multicolumn{11}{|c|}{ SEÑAL ORIGINAL } \\
\hline $0-300$ & 269.57 & 337.39 & 277.54 & 0 & 565.25 & 669.67 & 1009.9 & 456.34 & 996.78 & 728.11 & 0 \\
\hline $300-600$ & 38.324 & 47.013 & 38.865 & 0 & 50.5 & 31.01 & 43.934 & 33.085 & 94.332 & 50.346 & 0 \\
\hline $600-1200$ & 86.474 & 104.81 & 89.093 & 0 & 92.409 & 80.778 & 94.501 & 89.541 & 145.27 & 114.84 & 0 \\
\hline $\begin{array}{l}\text { BANDAS DE } \\
\text { FRECUENCIA }\end{array}$ & \multicolumn{11}{|c|}{ POTENCIAS DE LAS SEÑALES DE SONIDO RESPIRATORIO ESTIMADO SIN TW } \\
\hline $0-300$ & 243.72 & 206.37 & 221.93 & 0 & 492.63 & 353.12 & 863 & 326.53 & 656 & 573.48 & 0 \\
\hline $300-600$ & 18.183 & 14.159 & 13.313 & 0 & 23.654 & 20.364 & 19.529 & 16.648 & 38.26 & 41.965 & 0 \\
\hline $600-1200$ & 2.5653 & 1.8376 & 1.9689 & 0 & 4.2228 & 8.9495 & 3.114 & 5.4285 & 12.502 & 15.649 & 0 \\
\hline $\begin{array}{l}\text { BANDAS DE } \\
\text { FRECUENCIA }\end{array}$ & \multicolumn{11}{|c|}{ POTENCIAS DE LAS SEÑALES DE SONIDO RESPIRATORIO ESTIMADO CON TW } \\
\hline $0-300$ & 248.67 & 206.18 & 217.25 & 0 & 584.98 & 385.47 & 832.5 & 342.16 & 708.82 & 568.08 & 0 \\
\hline $300-600$ & 28.71 & 14.107 & 13.486 & 0 & 44.475 & 22.442 & 53.27 & 35.9 & 56.012 & 56.544 & 0 \\
\hline $600-1200$ & 5.5149 & 1.8546 & 2.4947 & 0 & 10.844 & 12.694 & 14.03 & 11.184 & 18.689 & 25.626 & 0 \\
\hline $\begin{array}{l}\text { BANDAS DE } \\
\text { FRECUENCIA }\end{array}$ & \multicolumn{11}{|c|}{ POTENCIAS DE RUIDO CARDIACO ESTIMADO SIN TW } \\
\hline $0-300$ & 81.042 & 167.55 & 133.79 & 0 & 290.53 & 459.63 & 260.69 & 264.32 & 580.75 & 467.02 & 0 \\
\hline $300-600$ & 1.9485 & 1.1852 & 1.2774 & 0 & 9.8095 & 14.447 & 8.9029 & 9.6819 & 22.61 & 33.106 & 0 \\
\hline $600-1200$ & 0.58041 & 0.44845 & 0.51252 & 0 & 3.0121 & 8.2707 & 2.0746 & 5.1918 & 10.257 & 15.084 & 0 \\
\hline $\begin{array}{l}\text { BANDAS DE } \\
\text { FRECUENCIA }\end{array}$ & \multicolumn{11}{|c|}{ POTENCIAS DE RUIDO CARDIACO ESTIMADO CON TW } \\
\hline $0-300$ & 96.483 & 168.38 & 136.28 & 0 & 315.92 & 448.12 & 256.67 & 255.01 & 612.99 & 441.71 & 0 \\
\hline $300-600$ & 19.668 & 1.473 & 2.1128 & 0 & 35.527 & 19.798 & 45.47 & 31.082 & 46.546 & 52.375 & 0 \\
\hline $600-1200$ & 4.1561 & 0.5004 & 1.2706 & 0 & 10.274 & 12.491 & 13.497 & 10.832 & 17.429 & 25.458 & 0 \\
\hline
\end{tabular}

Tabla 5. 6.d. Potencias calculadas de la señal en el dominio original obtenida de la línea axilar derecha apical para un flujo de $2.0 \mathrm{~L} / \mathrm{seg}$. 


\begin{tabular}{|c|c|c|c|c|c|c|c|c|c|c|c|}
\hline \multirow{4}{*}{ INTERVALOS } & \multicolumn{11}{|c|}{ DER2 } \\
\hline & \multicolumn{4}{|c|}{ APNEA } & \multicolumn{7}{|c|}{ ZONA DE RESPIRACIÓN } \\
\hline & 0 & 0 & 0 & 0 & 0 & 0 & 0 & 0 & 0 & 0 & 0 \\
\hline & 0 & 0 & 0 & 0 & 0 & 0 & 0 & 0 & 0 & 0 & 0 \\
\hline $\begin{array}{l}\text { BANDAS DE } \\
\text { FRECUENCIA }\end{array}$ & \multicolumn{11}{|c|}{ SEÑAL ORIGINAL } \\
\hline $0-300$ & 0 & 0 & 0 & 0 & 0 & 0 & 0 & 0 & 0 & 0 & 0 \\
\hline $300-600$ & 0 & 0 & 0 & 0 & 0 & 0 & 0 & 0 & 0 & 0 & 0 \\
\hline $600-1200$ & 0 & 0 & 0 & 0 & 0 & 0 & 0 & 0 & 0 & 0 & 0 \\
\hline $\begin{array}{l}\text { BANDAS DE } \\
\text { FRECUENCIA }\end{array}$ & \multicolumn{11}{|c|}{ POTENCIAS DE LAS SEÑALES DE SONIDO RESPIRATORIO ESTIMADO SIN TW } \\
\hline $0-300$ & 0 & 0 & 0 & 0 & 0 & 0 & 0 & 0 & 0 & 0 & 0 \\
\hline $300-600$ & 0 & 0 & 0 & 0 & 0 & 0 & 0 & 0 & 0 & 0 & 0 \\
\hline $600-1200$ & 0 & 0 & 0 & 0 & 0 & 0 & 0 & 0 & 0 & 0 & 0 \\
\hline $\begin{array}{l}\text { BANDAS DE } \\
\text { FRECUENCIA }\end{array}$ & \multicolumn{11}{|c|}{ POTENCIAS DE LAS SEÑALES DE SONIDO RESPIRATORIO ESTIMADO CON TW } \\
\hline $0-300$ & 0 & 0 & 0 & 0 & 0 & 0 & 0 & 0 & 0 & 0 & 0 \\
\hline $300-600$ & 0 & 0 & 0 & 0 & 0 & 0 & 0 & 0 & 0 & 0 & 0 \\
\hline $600-1200$ & 0 & 0 & 0 & 0 & 0 & 0 & 0 & 0 & 0 & 0 & 0 \\
\hline $\begin{array}{l}\text { BANDAS DE } \\
\text { FRECUENCIA }\end{array}$ & \multicolumn{11}{|c|}{ POTENCIAS DE RUIDO CARDIACO ESTIMADO SIN TW } \\
\hline 0-300 & 0 & 0 & 0 & 0 & 0 & 0 & 0 & 0 & 0 & 0 & 0 \\
\hline $300-600$ & 0 & 0 & 0 & 0 & 0 & 0 & 0 & 0 & 0 & 0 & 0 \\
\hline $600-1200$ & 0 & 0 & 0 & 0 & 0 & 0 & 0 & 0 & 0 & 0 & 0 \\
\hline $\begin{array}{l}\text { BANDAS DE } \\
\text { FRECUENCIA }\end{array}$ & \multicolumn{11}{|c|}{ POTENCIAS DE RUIDO CARDIACO ESTIMADO CON TW } \\
\hline $0-300$ & 0 & 0 & 0 & 0 & 0 & 0 & 0 & 0 & 0 & 0 & 0 \\
\hline $300-600$ & 0 & 0 & 0 & 0 & 0 & 0 & 0 & 0 & 0 & 0 & 0 \\
\hline $600-1200$ & 0 & 0 & 0 & 0 & 0 & 0 & 0 & 0 & 0 & 0 & 0 \\
\hline
\end{tabular}

Tabla 5. 6.e. Potencias calculadas de la señal en el dominio original obtenida de la línea axilar derecha media para un flujo de $2.0 \mathrm{~L} / \mathrm{seg}$.

\begin{tabular}{|c|c|c|c|c|c|c|c|c|c|c|c|}
\hline \multirow{4}{*}{ INTERVALOS } & \multicolumn{11}{|c|}{ DER3 } \\
\hline & \multicolumn{4}{|c|}{ APNEA } & \multicolumn{7}{|c|}{ ZONA DE RESPIRACIÓN } \\
\hline & 0 & 0 & 0 & 0 & 0 & 0 & 0 & 0 & 0 & 0 & 0 \\
\hline & 0 & 0 & 0 & 0 & 0 & 0 & 0 & 0 & 0 & 0 & 0 \\
\hline \multicolumn{12}{|l|}{$\begin{array}{l}\text { BANDAS DE } \\
\text { FRECUENCIA }\end{array}$} \\
\hline $0-300$ & 0 & 0 & 0 & 0 & 0 & 0 & 0 & 0 & 0 & 0 & 0 \\
\hline $300-600$ & 0 & 0 & 0 & 0 & 0 & 0 & 0 & 0 & 0 & 0 & 0 \\
\hline $600-1200$ & 0 & 0 & 0 & 0 & 0 & 0 & 0 & 0 & 0 & 0 & 0 \\
\hline $\begin{array}{l}\text { BANDAS DE } \\
\text { FRECUENCIA }\end{array}$ & \multicolumn{11}{|c|}{ POTENCIAS DE LAS SEÑALES DE SONIDO RESPIRATORIO ESTIMADO SIN TW } \\
\hline $0-300$ & 0 & 0 & 0 & 0 & 0 & 0 & 0 & 0 & 0 & 0 & 0 \\
\hline $300-600$ & 0 & 0 & 0 & 0 & 0 & 0 & 0 & 0 & 0 & 0 & 0 \\
\hline $600-1200$ & 0 & 0 & 0 & 0 & 0 & 0 & 0 & 0 & 0 & 0 & 0 \\
\hline $\begin{array}{l}\text { BANDAS DE } \\
\text { FRECUENCIA }\end{array}$ & \multicolumn{11}{|c|}{ POTENCIAS DE LAS SEÑALES DE SONIDO RESPIRATORIO ESTIMADO CON TW } \\
\hline $0-300$ & 0 & 0 & 0 & 0 & 0 & 0 & 0 & 0 & 0 & 0 & 0 \\
\hline $300-600$ & 0 & 0 & 0 & 0 & 0 & 0 & 0 & 0 & 0 & 0 & 0 \\
\hline $600-1200$ & 0 & 0 & 0 & 0 & 0 & 0 & 0 & 0 & 0 & 0 & 0 \\
\hline $\begin{array}{l}\text { BANDAS DE } \\
\text { FRECUENCIA }\end{array}$ & \multicolumn{11}{|c|}{ POTENCIAS DE RUIDO CARDIACO ESTIMADO SIN TW } \\
\hline $0-300$ & 0 & 0 & 0 & 0 & 0 & 0 & 0 & 0 & 0 & 0 & 0 \\
\hline $300-600$ & 0 & 0 & 0 & 0 & 0 & 0 & 0 & 0 & 0 & 0 & 0 \\
\hline $600-1200$ & 0 & 0 & 0 & 0 & 0 & 0 & 0 & 0 & 0 & 0 & 0 \\
\hline $\begin{array}{l}\text { BANDAS DE } \\
\text { FRECUENCIA }\end{array}$ & \multicolumn{11}{|c|}{ POTENCIAS DE RUIDO CARDIACO ESTIMADO CON TW } \\
\hline $0-300$ & 0 & 0 & 0 & 0 & 0 & 0 & 0 & 0 & 0 & 0 & 0 \\
\hline $300-600$ & 0 & 0 & 0 & 0 & 0 & 0 & 0 & 0 & 0 & 0 & 0 \\
\hline $600-1200$ & 0 & 0 & 0 & 0 & 0 & 0 & 0 & 0 & 0 & 0 & \\
\hline
\end{tabular}

Tabla 5. 6.f. Potencias calculadas de la señal en el dominio original obtenida de la línea axilar derecha apical para un flujo de $2.0 \mathrm{~L} / \mathrm{seg}$. 


\begin{tabular}{|c|c|c|c|c|c|c|c|c|c|c|c|}
\hline \multirow{4}{*}{ INTERVALOS } & \multicolumn{11}{|c|}{ MIT } \\
\hline & \multicolumn{4}{|c|}{ APNEA } & \multicolumn{7}{|c|}{ ZONA DE RESPIRACIÓN } \\
\hline & 626 & 3241 & 6127 & 8817 & 11758 & 14483 & 17326 & 20022 & 23146 & 25588 & 36811 \\
\hline & 1422 & 4466 & 7352 & 10084 & 13116 & 15708 & 18122 & 20818 & 23942 & 26837 & 38036 \\
\hline $\begin{array}{l}\text { BANDAS DE } \\
\text { FRECUENCIA }\end{array}$ & \multicolumn{11}{|c|}{ SEÑAL ORIGINAL } \\
\hline $0-300$ & 734.03 & 854.25 & 865.9 & 948.76 & 1318.4 & 1723.5 & 782.13 & 687.32 & 915.92 & 1707.4 & 2004.9 \\
\hline $300-600$ & 68.575 & 83.61 & 70.692 & 71.328 & 107.23 & 65.701 & 54.437 & 51.717 & 69.689 & 81.16 & 74.746 \\
\hline $600-1200$ & 147.77 & 185.26 & 181.73 & 191.68 & 210.1 & 173.31 & 128.44 & 120.92 & 131.71 & 186.55 & 182.21 \\
\hline $\begin{array}{l}\text { BANDAS DE } \\
\text { FRECUENCIA }\end{array}$ & \multicolumn{11}{|c|}{ POTENCIAS DE LAS SEÑALES DE SONIDO RESPIRATORIO ESTIMADO SIN TW } \\
\hline $0-300$ & 617.76 & 667.56 & 487.22 & 710.75 & 868.86 & 1007.2 & 488.54 & 511.7 & 674.34 & 1144.4 & 1447 \\
\hline $300-600$ & 7.9314 & 54.58 & 16.321 & 61.162 & 30.981 & 36.383 & 25.717 & 8.2312 & 25.995 & 44.414 & 35.393 \\
\hline $600-1200$ & 2.6359 & 5.9287 & 7.2099 & 10.731 & 15.291 & 16.438 & 4.5533 & 2.354 & 7.5287 & 9.9049 & 8.7977 \\
\hline $\begin{array}{c}\text { BANDAS DE } \\
\text { FRECUENCIA }\end{array}$ & \multicolumn{11}{|c|}{ POTENCIAS DE LAS SEÑALES DE SONIDO RESPIRATORIO ESTIMADO CON TW } \\
\hline $0-300$ & 663 & 631.35 & 525.18 & 935.89 & 957.62 & 1009.7 & 616.36 & 502.63 & 651.4 & 1224.7 & 1491.1 \\
\hline $300-600$ & 20.28 & 48.948 & 77.115 & 269.21 & 88.36 & 97.567 & 84.046 & 14.393 & 53.198 & 187.12 & 114.82 \\
\hline $600-1200$ & 6.6546 & 6.1036 & 40.939 & 44.213 & 55.003 & 48.911 & 45.248 & 25.494 & 61.286 & 66.369 & 47.691 \\
\hline $\begin{array}{l}\text { BANDAS DE } \\
\text { FRECUENCIA }\end{array}$ & \multicolumn{11}{|c|}{ POTENCIAS DE RUIDO CARDIACO ESTIMADO SIN TW } \\
\hline $0-300$ & 271.55 & 700.05 & 729.82 & 901.01 & 875.76 & 1032.4 & 645.14 & 215.88 & 508.74 & 1309.8 & 941.88 \\
\hline $300-600$ & 5.8097 & 53.186 & 15.925 & 59.444 & 30.21 & 36.332 & 25.547 & 7.6633 & 26.502 & 43.768 & 35.286 \\
\hline $600-1200$ & 2.3725 & 5.8908 & 7.1569 & 10.592 & 15.259 & 16.444 & 4.5508 & 2.3566 & 7.4528 & 9.9149 & 8.7832 \\
\hline $\begin{array}{l}\text { BANDAS DE } \\
\text { FRECUENCIA }\end{array}$ & \multicolumn{11}{|c|}{ POTENCIAS DE RUIDO CARDIACO ESTIMADO CON TW } \\
\hline $0-300$ & 258.46 & 671.62 & 770.34 & 1107.9 & 854.65 & 1034.3 & 708.69 & 211.93 & 530.31 & 1395.5 & 1036.3 \\
\hline $300-600$ & 19.305 & 47.409 & 77.5 & 267.89 & 87.145 & 97.412 & 84.029 & 13.844 & 53.468 & 186.36 & 114.06 \\
\hline $600-1200$ & 6.5873 & 6.0649 & 40.875 & 44.109 & 54.979 & 48.902 & 45.233 & 25.496 & 61.303 & 66.359 & 47.643 \\
\hline
\end{tabular}

Tabla 5. 6.g. Potencias calculadas de la señal en el dominio original obtenida de la zona de auscultación de la válvula mitral para un flujo de $2.0 \mathrm{~L} / \mathrm{seg}$.

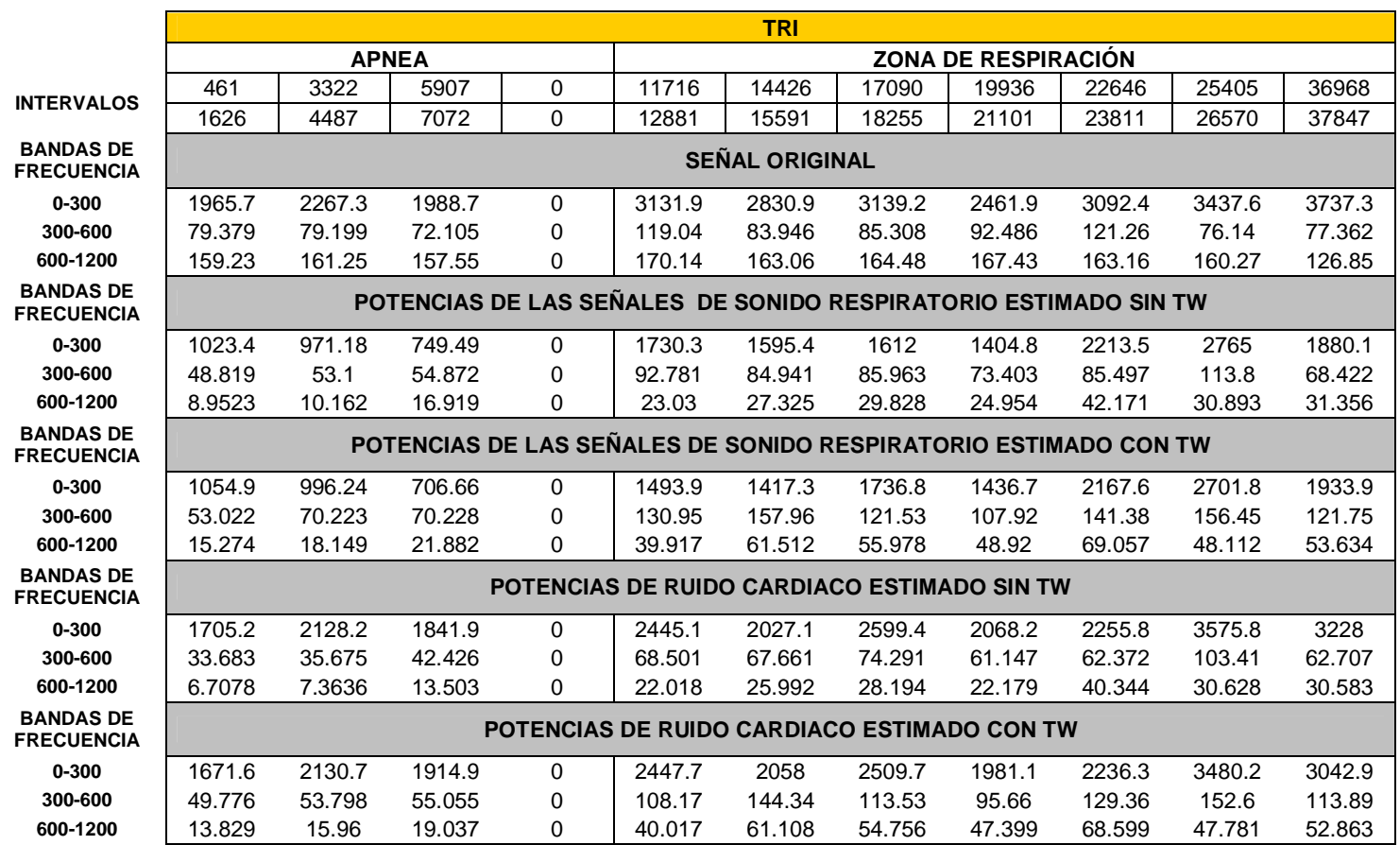

Tabla 5. 6.h. Potencias calculadas de la señal en el dominio original obtenida de la zona de auscultación de la válvula tricúspide para un flujo de $2.0 \mathrm{~L} / \mathrm{seg}$. 


\begin{tabular}{|c|c|c|c|c|c|c|c|c|c|c|c|}
\hline \multirow{4}{*}{ INTERVALOS } & \multicolumn{11}{|c|}{ PUL } \\
\hline & \multicolumn{4}{|c|}{ APNEA } & \multicolumn{7}{|c|}{ ZONA DE RESPIRACIÓN } \\
\hline & 671 & 3509 & 6244 & 0 & 0 & 14390 & 0 & 20045 & 22662 & 25410 & 0 \\
\hline & 2205 & 4583 & 7334 & 0 & 0 & 15464 & 0 & 20852 & 23736 & 26484 & 0 \\
\hline \multicolumn{12}{|l|}{$\begin{array}{l}\text { BANDAS DE } \\
\text { FRECUENCIA }\end{array}$} \\
\hline $0-300$ & 4720.1 & 3548.9 & 3504.9 & 0 & 0 & 8228 & 0 & 5796.4 & 11091 & 8791.5 & 0 \\
\hline $300-600$ & 159 & 121.36 & 101.55 & 0 & 0 & 186.12 & 0 & 76.257 & 497.38 & 163.06 & 0 \\
\hline $600-1200$ & 245.97 & 155.12 & 176.15 & 0 & 0 & 186 & 0 & 138.06 & 228.48 & 202.23 & 0 \\
\hline $\begin{array}{l}\text { BANDAS DE } \\
\text { FRECUENCIA }\end{array}$ & \multicolumn{11}{|c|}{ POTENCIAS DE LAS SEÑALES DE SONIDO RESPIRATORIO ESTIMADO SIN TW } \\
\hline $0-300$ & 3207.8 & 1971.9 & 2217.7 & 0 & 0 & 6166.6 & 0 & 5654.7 & 6883.7 & 6382.3 & 0 \\
\hline $300-600$ & 171.29 & 121.26 & 103.92 & 0 & 0 & 231.74 & 0 & 92.974 & 523.54 & 211.73 & 0 \\
\hline $600-1200$ & 190.54 & 123.69 & 132.43 & 0 & 0 & 145.67 & 0 & 93.082 & 191.38 & 175.01 & 0 \\
\hline $\begin{array}{l}\text { BANDAS DE } \\
\text { FRECUENCIA }\end{array}$ & \multicolumn{11}{|c|}{ POTENCIAS DE LAS SEÑALES DE SONIDO RESPIRATORIO ESTIMADO CON TW } \\
\hline $0-300$ & 3038.6 & 1970.2 & 2447.4 & 0 & 0 & 6212.3 & 0 & 5780.3 & 6837.7 & 6073.2 & 0 \\
\hline $300-600$ & 171.63 & 121.75 & 126.73 & 0 & 0 & 276.71 & 0 & 103.61 & 555.15 & 237.36 & 0 \\
\hline $600-1200$ & 190.65 & 123.64 & 133.11 & 0 & 0 & 173.31 & 0 & 99.713 & 215.28 & 185.97 & 0 \\
\hline $\begin{array}{l}\text { BANDAS DE } \\
\text { FRECUENCIA }\end{array}$ & \multicolumn{11}{|c|}{ POTENCIAS DE RUIDO CARDIACO ESTIMADO SIN TW } \\
\hline $0-300$ & 2358.2 & 2093.3 & 2200.3 & 0 & 0 & 3209.5 & 0 & 859.13 & 3448.8 & 3824 & 0 \\
\hline $300-600$ & 38.045 & 8.7947 & 30.502 & 0 & 0 & 110.73 & 0 & 37.734 & 104.86 & 114.24 & 0 \\
\hline $600-1200$ & 11.066 & 1.9931 & 9.044 & 0 & 0 & 22.287 & 0 & 8.7076 & 35.677 & 54.35 & 0 \\
\hline $\begin{array}{l}\text { BANDAS DE } \\
\text { FRECUENCIA }\end{array}$ & \multicolumn{11}{|c|}{ POTENCIAS DE RUIDO CARDIACO ESTIMADO CON TW } \\
\hline $0-300$ & 2273.1 & 2091.9 & 2312.4 & 0 & 0 & 3429 & 0 & 832.68 & 3207.2 & 3993.1 & 0 \\
\hline $300-600$ & 41.015 & 7.3946 & 58.855 & 0 & 0 & 192.23 & 0 & 55.652 & 177.36 & 167.78 & 0 \\
\hline $600-1200$ & 20.056 & 1.4865 & 19.746 & 0 & 0 & 78.889 & 0 & 31.716 & 95.214 & 96.626 & 0 \\
\hline
\end{tabular}

Tabla 5. 6.i. Potencias calculadas de la señal en el dominio original obtenida de la zona de auscultación de la válvula pulmonar para un flujo de $2.0 \mathrm{~L} / \mathrm{seg}$.

\begin{tabular}{|c|c|c|c|c|c|c|c|c|c|c|c|}
\hline \multirow{4}{*}{ INTERVALOS } & \multicolumn{11}{|c|}{ AOR } \\
\hline & \multicolumn{4}{|c|}{ APNEA } & \multicolumn{7}{|c|}{ ZONA DE RESPIRACIÓN } \\
\hline & 536 & 3325 & 6227 & 8944 & 11854 & 14562 & 16996 & 20119 & 22512 & 25216 & 36731 \\
\hline & 1563 & 4448 & 7033 & 10077 & 12400 & 15638 & 18023 & 21146 & 23539 & 26401 & 37527 \\
\hline \multicolumn{12}{|l|}{$\begin{array}{l}\text { BANDAS DE } \\
\text { FRECUENCIA }\end{array}$} \\
\hline $0-300$ & 1553.2 & 1745.6 & 1410.8 & 1810.3 & 1855.1 & 4700 & 2930.8 & 2403.5 & 3860.6 & 3522.2 & 2767.1 \\
\hline $300-600$ & 66.417 & 81.642 & 41.398 & 61.541 & 157.18 & 93.21 & 98.973 & 161.14 & 320.92 & 92.873 & 56.024 \\
\hline $600-1200$ & 133.72 & 148.76 & 101.42 & 142.55 & 108.11 & 156.96 & 151.41 & 145.23 & 178.71 & 152.83 & 103.02 \\
\hline $\begin{array}{l}\text { BANDAS DE } \\
\text { FRECUENCIA }\end{array}$ & \multicolumn{11}{|c|}{ POTENCIAS DE LAS SEÑALES DE SONIDO RESPIRATORIO ESTIMADO SIN TW } \\
\hline $0-300$ & 921.74 & 2426.3 & 907.83 & 1675.5 & 1632.8 & 4170.6 & 3053.8 & 3994.4 & 4532.8 & 5190.1 & 4014.4 \\
\hline $300-600$ & 83.696 & 629.54 & 61.501 & 106.09 & 173.13 & 479.17 & 259.04 & 1849.1 & 2873.8 & 2573.9 & 2404.9 \\
\hline $600-1200$ & 109.92 & 951.65 & 95.656 & 135.8 & 94.576 & 531.29 & 225.26 & 3061.7 & 4448.8 & 4194.5 & 2193.7 \\
\hline $\begin{array}{l}\text { BANDAS DE } \\
\text { FRECUENCIA }\end{array}$ & \multicolumn{11}{|c|}{ POTENCIAS DE LAS SEÑALES DE SONIDO RESPIRATORIO ESTIMADO CON TW } \\
\hline $0-300$ & 903.95 & 2441.3 & 921.59 & 1621.4 & 1554.4 & 4065.4 & 2829.7 & 4721.4 & 4853.3 & 4942.1 & 4394.6 \\
\hline $300-600$ & 87.642 & 701.71 & 74.627 & 110.33 & 168.82 & 576.97 & 222.48 & 2157.6 & 3195.5 & 2895.9 & 2807.8 \\
\hline $600-1200$ & 110.73 & 1002 & 101.31 & 138.19 & 93.305 & 642.02 & 213.9 & 3364.8 & 4831.1 & 4593.6 & 2448.7 \\
\hline $\begin{array}{l}\text { BANDAS DE } \\
\text { FRECUFNCIA }\end{array}$ & \multicolumn{11}{|c|}{ POTENCIAS DE RUIDO CARDIACO ESTIMADO SIN TW } \\
\hline $0-300$ & 1108.2 & 1897.4 & 729.22 & 957.12 & 387.72 & 3617.3 & 2452.3 & 4519.9 & 4226.8 & 4771.8 & 2699.9 \\
\hline $300-600$ & 32.713 & 583.7 & 31.162 & 62.716 & 26.343 & 447.7 & 201.72 & 1761.9 & 2678.8 & 2541.4 & 2372.4 \\
\hline $600-1200$ & 25.74 & 872.66 & 18.522 & 49.299 & 21.451 & 466.6 & 153.43 & 3009.8 & 4382.2 & 4137.1 & 2144.1 \\
\hline $\begin{array}{l}\text { BANDAS DE } \\
\text { FRECUENCIA }\end{array}$ & \multicolumn{11}{|c|}{ POTENCIAS DE RUIDO CARDIACO ESTIMADO CON TW } \\
\hline $0-300$ & 1120.4 & 1870.6 & 746.18 & 1020.7 & 420.47 & 3725 & 2322.2 & 5139.4 & 4602.5 & 4415 & 3113.6 \\
\hline $300-600$ & 40.125 & 668.96 & 46.144 & 70.634 & 25.691 & 545.75 & 180.47 & 2120.4 & 3085.8 & 2860.1 & 2800.3 \\
\hline $600-1200$ & 31.837 & 934.78 & 25.834 & 53.441 & 21.164 & 595.53 & 143.84 & 3351.5 & 4797.2 & 4556.6 & 2430.7 \\
\hline
\end{tabular}

Tabla 5. 6.j. Potencias calculadas de la señal en el dominio original obtenida de la zona de auscultación de la válvula aórtica para un flujo de $2.0 \mathrm{~L} / \mathrm{seg}$. 


\begin{tabular}{|c|c|c|c|c|c|c|c|c|c|c|c|}
\hline \multirow{4}{*}{ INTERVALOS } & \multicolumn{11}{|c|}{ PRE } \\
\hline & \multicolumn{4}{|c|}{ APNEA } & \multicolumn{7}{|c|}{ ZONA DE RESPIRACIÓN } \\
\hline & 630 & 3403 & 6106 & 8974 & 11486 & 14179 & 17080 & 19977 & 22909 & 25439 & 36957 \\
\hline & 1703 & 4911 & 7179 & 10246 & 13156 & 15810 & 18050 & 20947 & 23999 & 26746 & 38030 \\
\hline $\begin{array}{l}\text { BANDAS DE } \\
\text { FRECUENCIA }\end{array}$ & \multicolumn{11}{|c|}{ SEÑAL ORIGINAL } \\
\hline $0-300$ & 1067.2 & 1208.7 & 960.1 & 1249.3 & 2151.3 & 2259.1 & 919.83 & 1089.7 & 1656.4 & 2158.1 & 1745.8 \\
\hline $300-600$ & 65.092 & 95.73 & 60.486 & 72.871 & 119.51 & 130.3 & 50.116 & 73.575 & 220.45 & 95.357 & 57.901 \\
\hline $600-1200$ & 172.68 & 221.83 & 148.93 & 189.57 & 245.97 & 240.76 & 130.71 & 154.38 & 223.48 & 193.14 & 160.78 \\
\hline $\begin{array}{l}\text { BANDAS DE } \\
\text { FRECUENCIA }\end{array}$ & \multicolumn{11}{|c|}{ POTENCIAS DE LAS SEÑALES DE SONIDO RESPIRATORIO ESTIMADO SIN TW } \\
\hline $0-300$ & 831.67 & 1087.1 & 809.93 & 888.67 & 1894.5 & 1718.2 & 655.85 & 920.07 & 1240.1 & 1660.7 & 1553.2 \\
\hline $300-600$ & 29.252 & 69.363 & 16.418 & 50.033 & 136.09 & 138.06 & 49.451 & 49.151 & 71.941 & 185.2 & 73.197 \\
\hline $600-1200$ & 36.039 & 88.942 & 21.578 & 50.848 & 125.59 & 102.36 & 29.503 & 24.124 & 60.798 & 309.16 & 29.736 \\
\hline $\begin{array}{l}\text { BANDAS DE } \\
\text { FRECUENCIA }\end{array}$ & \multicolumn{11}{|c|}{ POTENCIAS DE LAS SEÑALES DE SONIDO RESPIRATORIO ESTIMADO CON TW } \\
\hline $0-300$ & 836.27 & 1089.4 & 813.66 & 1005.4 & 2010.7 & 2085.2 & 688.22 & 867.08 & 1343 & 1891.7 & 1360 \\
\hline $300-600$ & 36.002 & 97.737 & 13.143 & 174.84 & 325.8 & 308.33 & 84.971 & 73.74 & 221.22 & 320.8 & 140.96 \\
\hline $600-1200$ & 47.529 & 122.68 & 18.881 & 122.65 & 194.1 & 199.52 & 44.356 & 36.598 & 179.51 & 321.74 & 71.109 \\
\hline $\begin{array}{l}\text { BANDAS DE } \\
\text { FRECUENCIA }\end{array}$ & \multicolumn{11}{|c|}{ POTENCIAS DE RUIDO CARDIACO ESTIMADO SIN TW } \\
\hline $0-300$ & 504.64 & 722.36 & 420.63 & 1020.1 & 1378.2 & 1799.4 & 611.52 & 678.05 & 893.18 & 1771.5 & 1117.8 \\
\hline $300-600$ & 26.263 & 63.736 & 11.902 & 47.911 & 133.07 & 137.21 & 47.724 & 48.726 & 56.404 & 185.2 & 70.801 \\
\hline $600-1200$ & 35.888 & 88.302 & 21.361 & 50.566 & 125.2 & 101.98 & 29.454 & 24.057 & 58.801 & 309.07 & 29.864 \\
\hline $\begin{array}{l}\text { BANDAS DE } \\
\text { FRECUENCIA }\end{array}$ & \multicolumn{11}{|c|}{ POTENCIAS DE RUIDO CARDIACO ESTIMADO CON TW } \\
\hline $0-300$ & 501.19 & 712.05 & 417.39 & 1147.1 & 1596 & 1982.3 & 615.72 & 809.48 & 960.37 & 1902.2 & 1291.8 \\
\hline $300-600$ & 34.312 & 92.57 & 8.7822 & 174.85 & 323.13 & 306.57 & 84.692 & 73.058 & 215.77 & 321.02 & 140.24 \\
\hline $600-1200$ & 47.509 & 122.18 & 18.782 & 122.71 & 194.1 & 199.29 & 44.372 & 36.583 & 178.65 & 321.55 & 71.056 \\
\hline
\end{tabular}

Tabla 5. 6.k. Potencias calculadas de la señal en el dominio original obtenida de la zona de auscultación precordial promedio para un flujo de $2.0 \mathrm{~L} / \mathrm{seg}$. 

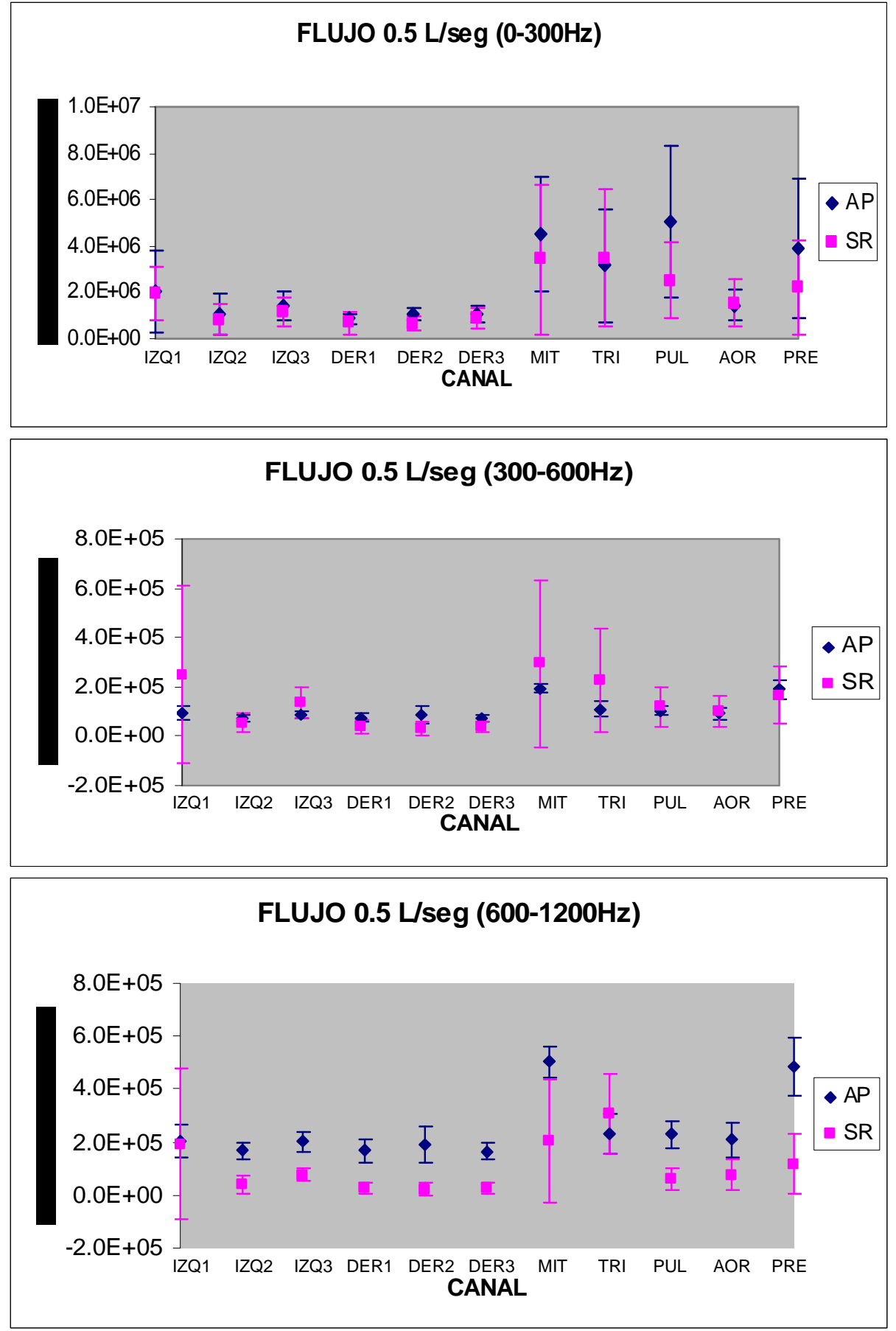

Figura 5.51 Potencias promedio de todos los sujetos a flujo de $0.5 \mathrm{~L} /$ seg en diferentes bandas de frecuencia. 

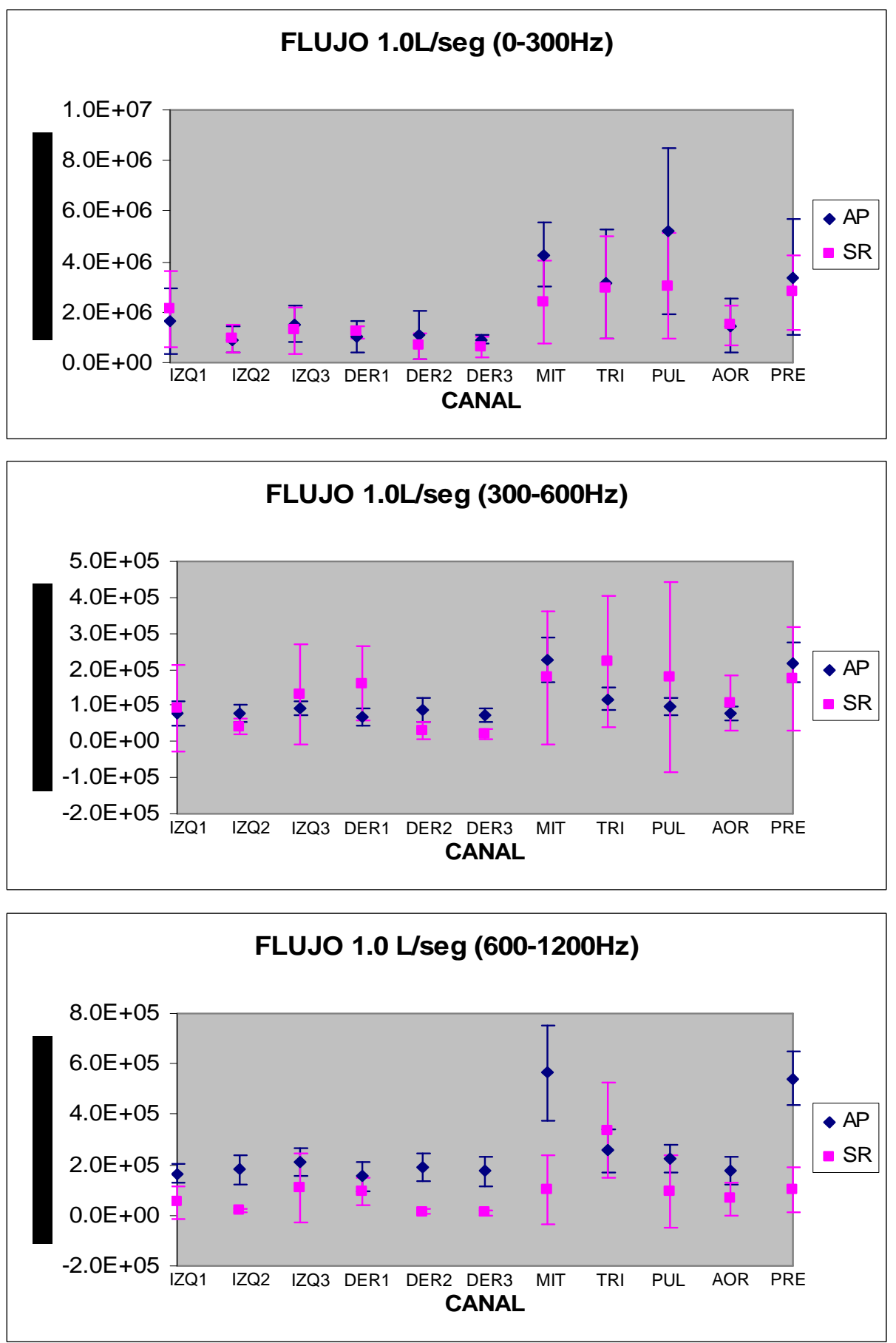

Figura 5.52 Potencias promedio de todos los sujetos a flujo de 1.0L/seg en diferentes bandas de frecuencia. 

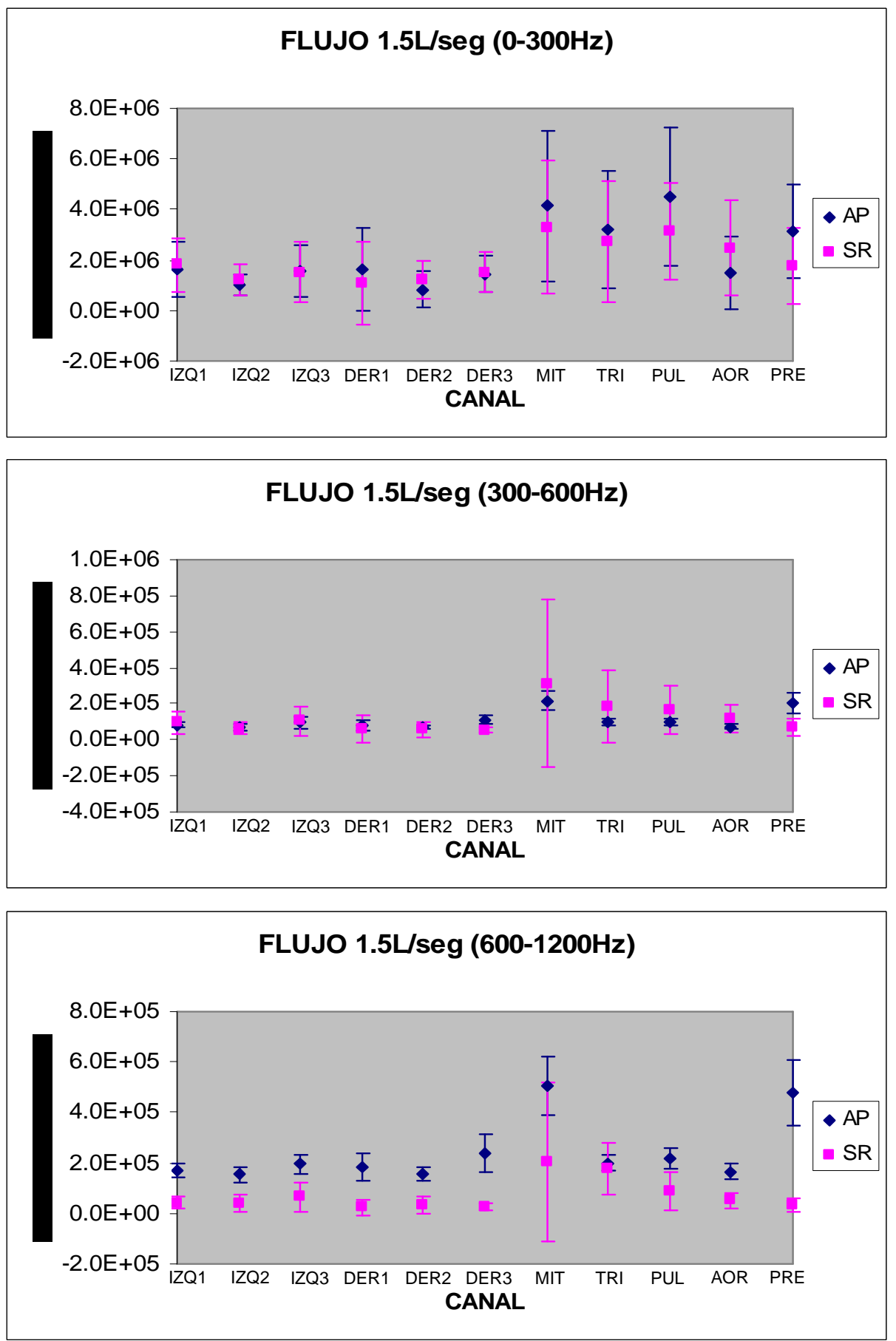

Figura 5.53 Potencias promedio de todos los sujetos a flujo de 1.5L/seg en diferentes bandas de frecuencia 

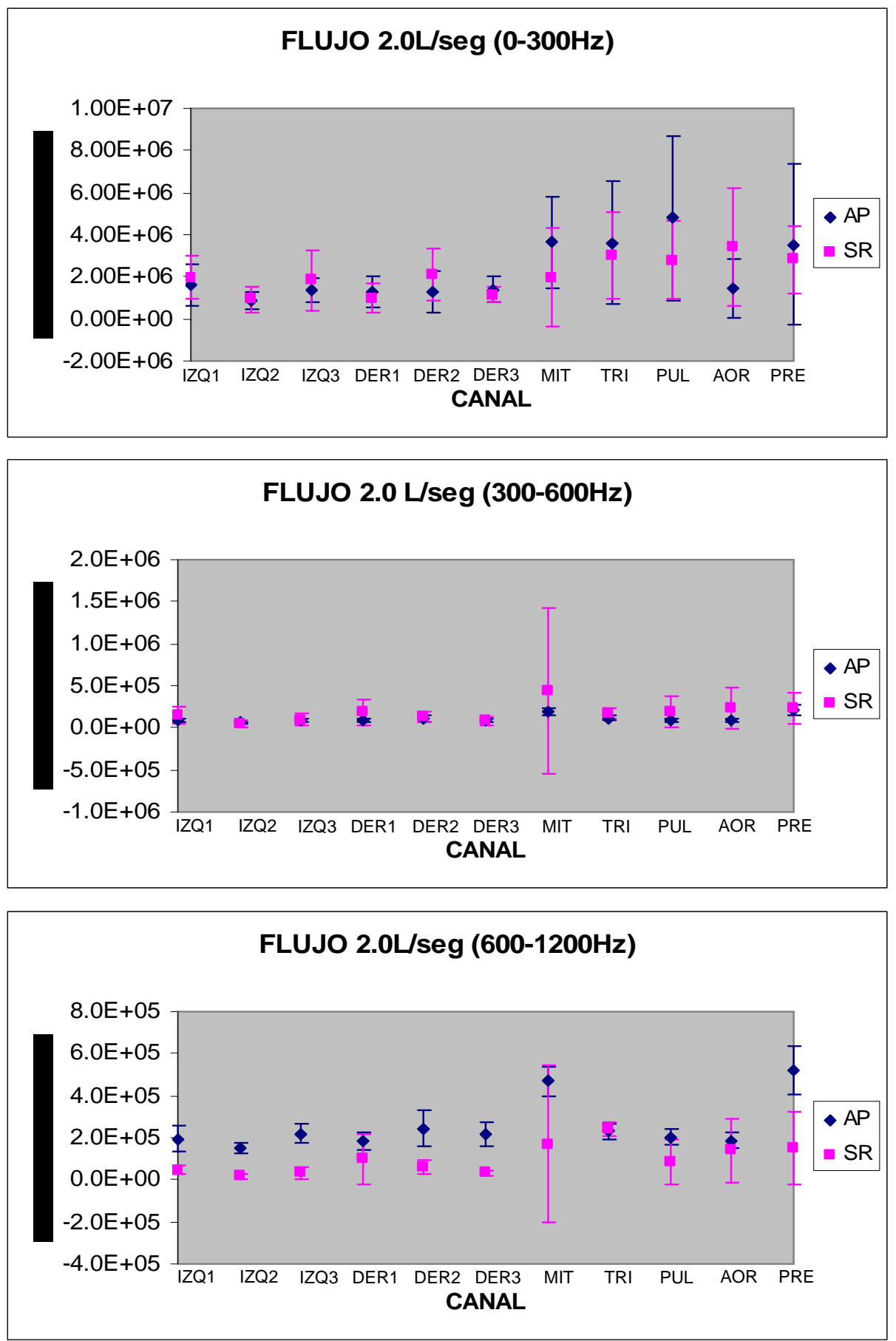

Figura 5.54 Potencias promedio de todos los sujetos a flujo de 2.0L/seg en diferentes bandas de frecuencia. 


\section{CAPÍTULO 6 DISCUSIÓN Y CONCLUSIONES}

\subsection{Análisis Cualitativo de la Morfología de las Señales Acústicas Torácicas Adquiridas.}

Los primeros esfuerzos en el análisis cuantitativo de los sonidos respiratorios, utilizaron básicamente un solo micrófono para la obtención de información acústica [13]. Sin embargo, el interés actual es extender el análisis del sonido respiratorio a toda la superficie torácica, vía su adquisición de forma simultánea mediante sistemas multicanales [25]. El problema en la adquisición de múltiples canales es la magnitud de la interferencia producida por los ruidos cardiacos la cual varía de punto a punto sobre la superficie, éste hecho se puede observar en un ejemplo de las señales obtenidas en la Figura 4.15. En los registros cercanos al miocardio se aprecian ruidos cardiacos con mayor intensidad, mientras que en las señales obtenidas en las llamadas líneas axilares existe pérdida de la intensidad acústica cardiaca, que se acentúa en los registros que corresponden a la línea axilar derecha. Esta observación se mantiene para señales acústicas registradas a todos los flujos respiratorios que se emplearon en este trabajo. Los registros que definen en mayor medida las características de los ruidos cardiacos, en cuanto a amplitud y morfología, son los correspondientes a la zona de auscultación de las válvulas cardiacas, sobre todo las válvula tricúspide y válvula mitral.

En la etapa de apnea, en donde la señal de flujo respiratorio es igual a cero, el ruido cardiaco se encuentra presente con diferente magnitud y morfología dependiendo de la cercanía del micrófono al corazón. Es posible observar una ligera variación de la morfología entre ruidos cardiacos a lo largo del tiempo en esta etapa sin embargo, en la etapa de respiración se puede advertir un incremento en la intensidad y un cambio en la morfología del ruido cardiaco en todos los canales. Observe en particular la señal etiquetada como IZQ3, a la izquierda de la Figura 4.15. En otros ejemplos de señales adquiridas a flujos respiratorios diferentes se observa un cambio de morfología, incluyendo un incremento en la intensidad, del ruido cardiaco con el flujo respiratorio en todos los canales, Figura 5.9, 5.10, 5.11 y 5.12. Esta observación parece confirmar el efecto que tiene la respiración sobre los ruidos cardiacos [10]. El comportamiento anteriormente descrito, se cumple en los diferentes sujetos adquiridos. Hay que hacer 
notar que en los escenarios simulados en esta tesis se utilizaron diferentes primeros ruidos cardiacos adquiridos, uno de ellos se insertó en la etapa de apnea y otro diferente en la etapa de respiración para incluir la observación anterior.

Con base en la observación anterior, un filtrado de tipo determinístico no sería adecuado dado que las características del filtrado de los ruidos cardiacos probablemente no serían igualmente aplicables a todos los canales de registro, y parámetros como la frecuencia de corte se tendrían que modificar canal por canal. Además, el mencionado traslape en frecuencia entre la información cardiaca y respiratoria excluye esta forma de procesamiento.

Con relación al sonido respiratorio, éste se incrementa en magnitud en mayor medida en la fase inspiratoria que en la espiratoria, en todas las posiciones adquiridas conforme el flujo respiratorio se incrementa de 0.5 a 2 L/seg. Este hecho hace que la detección de los ruidos cardiacos se complique en ciertas posiciones, por decremento de la relación señal a ruido, por ejemplo obsérvese la Figura 5.13.

\subsection{EMD en el Procesamiento de las Señales Acústicas Adquiridas: Análisis Cualitativo de los Modos de Oscilación.}

El algoritmo de descomposición Empirical Mode Descomposition (EMD) es un método adaptativo que permite analizar señales no estacionarias y no lineales (ver apéndices). El algoritmo ha sido aplicado a señales fisiológicas de naturaleza eléctrica como por ejemplo en señales de electrogastrograma [27] y en electrocardiogramas para analizar la variabilidad en frecuencia cardiaca [28]. Sin embargo, el EMD no se había aplicado en señales acústicas para el análisis de los sonidos respiratorios. En la técnica de descomposición no es necesario tener un conocimiento estadístico previo de las señales involucradas o determinar un número de sub-bandas a obtener como lo requiere la transformada wavelet en la propuesta de Charleston et. al [21].

Las señales simuladas permitieron obtener un conocimiento del análisis por EMD de señales acústicas bajo condiciones de diferentes relaciones de magnitud entre la señal respiratoria, el ruido cardiaco y el ruido de fondo. Por ejemplo, en las señales 
simuladas de 50 y $20 \mathrm{~dB}$ se obtienen varios IMFs, sin embargo y dependiendo del nivel de ruido de fondo, los primeros IMFs, IMF1 en nuestros resultados, pueden contener solamente información de alta oscilación y de amplitud constante, en donde no se observan cambios de amplitud del IMF con el flujo respiratorio. Si el ruido de fondo disminuye, desde los primeros IMFs se presenta información de los sonidos respiratorios ya que se observan cambios de amplitud de los IMFs correspondientes con la fases inspiratorias y espiratorias de la señal de flujo respiratorio. Conforme se obtienen IMFs adicionales comienzan los componentes de los ruidos cardiacos, que se detectan visualmente por su correspondencia temporal con la información en la señal original. Nótese que en las diferentes Figura 5.3 a 5.8, los ruidos cardiacos comparten escalas de tiempo con los sonidos respiratorios en todo momento. Es decir, en las simulaciones realizadas no se presentan de forma aislada componentes asociados exclusivamente al ruido cardiaco en los IMFs, si esto sucediera, los IMFs correspondientes podrían eliminarse de la lista para reducir la presencia del ruido en la señal final resultante de la recombinación de los IMFs restantes.

En las señales reales adquiridas a flujo respiratorios pequeños, como en las Figura 5.15 a $5.17,5.18$ a 5.20 y 5.21 a 5.23, las características que presentan los primeros IMFs, IMF1 hasta IMF4 en alguno de los casos, son alta oscilación y amplitud constante, siendo sus características similares a las características de los IMFs asociados al ruido de fondo en las señales simuladas. Sin embargo, cuando la señal adquirida se encuentra en posiciones cercanas al corazón, los primeros IMFs, después el IMF1 e IMF2, corresponden a ruidos cardiacos, dada la correspondencia temporal de la información contenida en ellos con la información en el domino original de la señal.

En comparación para señales acústicas que se obtienen con un flujo de 2 L/seg, Figura 5.24 a 5.26, aparecen componentes del sonido respiratorio y del ruido de fondo de igual forma en los primeros IMFs, sobre todo para las señales que se encuentran en la línea media axilar derecha; las características que presentan estos IMFs para determinar que pertenece al sonido respiratorio es la variación de su amplitud con respecto al flujo, es decir con la inspiración y espiración. Los componentes de los ruidos cardiacos son evidentes entre el quinto y octavo IMFs (sobre todo para las señales que 
se encuentran más cercanas al corazón), evidentes principalmente por sus características cuasi-periódicas.

Con respecto a los modos de oscilación de baja frecuencia, estos tienden a ser señales de tipo senoidal de amplitud pequeña, sin tener ninguna relación con las señales adquiridas, Figura 5.26.

\subsection{Análisis Cualitativo de las Señales Adquiridas vs. Filtradas por la Metodología Propuesta.}

Para hacer más fácil la visualización de resultados se han insertado en las figuras que presentan las señales sin filtrar y filtradas una serie de flechas para destacar aquellas posiciones en donde es evidente una reducción del ruido cardiaco en la etapa de respiración. Por ejemplo, en la Figura 5.36 que presenta la señal registrada en la posición en donde normalmente se ausculta la válvula tricúspide, las flechas señalan posiciones en donde se ha disminuido la presencia del primer ruido cardiaco. Este caso presenta condiciones de una buena SNR para el ruido cardiaco dado el flujo respiratorio pequeño de $0.5 \mathrm{~L} / \mathrm{seg}$. Otro escenario es el expuesto en la Figura 5.41 y 5.43 , en donde el flujo es de 2 L/seg y aún se observa una reducción de ruido cardiaco bajo estas condiciones.

La relación ruido cardiaco-señal respiratoria es un factor importante para localizar y minimizar los ruidos cardiacos, es decir, entre más pequeña sea dicha razón, disminuye la posibilidad de localizar los componentes cuasi-periódicos del ruido así como también minimizar su efecto. Un ejemplo se muestra en la línea axilar derecha, Figura 5.43, ya que en estos puntos la relación ruido cardiaco-señal respiratoria tiende a ser pequeña. Es por esta razón que en algunas tablas de posiciones detectadas de los ruidos cardiacos y potencias espectrales se presentan valores de posición cero dado que no pudieron detectar los ruidos cardiacos, ejemplo de ello son las Tabla 5. 3.d, 5.4.e y 5.5.d. Otra nota importante de subrayar es que en la metodología propuesta se intentó reducir sólo el primer ruido cardiaco, por lo que el segundo ruido cardiaco está presente en las señales filtradas. Sin embargo, un procedimiento similar se puede seguir para reducir el segundo ruido. 
Una observación importante que se realizó al aplicar la metodología propuesta, es la introducción de artefactos de alta frecuencia en la señal filtrada predominantemente cuando la metodología propuesta incluye el uso de TW en el procedimiento de eliminación del ruido cardiaco. Se descubrió que este fenómeno se debía a que el procedimiento no lineal de TW introduce pequeñas discontinuidades en la señal de referencia que se intenta alinear con la señal de interferencia, esto para poder ajustar las diferencias de morfología entre ambas señales. Cuando estas pequeñas discontinuidades pasan a través del filtro adaptativo, el cual trabaja de forma recursiva muestra a muestra de tiempo, estas discontinuidades representan cambios drásticos del tipo no estacionario con los cuales el algoritmo LMS no puede contender. Por lo tanto, la señal de interferencia estimada, señal $y$ en la Figura 4.9, presenta las discontinuidades mencionadas pero amplificadas que posteriormente pasan a la señal de error, que representa a la señal filtrada. La energía añadida se encuentra preponderantemente en la banda de alta frecuencia de $600-1200 \mathrm{~Hz}$.

\subsection{Desempeño de la Metodología Propuesta con Base en el Análisis de Potencias Espectrales.}

Una de las ventajas de utilizar señales simuladas para examinar el desempeño de la metodología propuesta es el hecho de que se conocen todos los elementos de las señales involucradas, por ejemplo, el número de ruidos cardiacos y su contenido en frecuencia original, los sonidos respiratorios y su contenido en frecuencia, la SNR, entre otras. De esta forma es posible evaluar el número de ruidos cardiacos de interferencia detectados y estimados, su potencia espectral en diferentes bandas y las posibles alteraciones al sonido respiratorio. Las observaciones, conclusiones, experiencia y conocimientos que se logran extraer a partir de escenarios simulados, pueden extrapolarse a señales reales en donde es difícil tener una referencia confiable del ruido cardiaco de interferencia.

En las Tabla 5.1 y 5.2 se presentan las potencias espectrales calculadas para dos condiciones diferentes de ruido de fondo en señales simuladas. Con relación al ruido cardiaco, se observa que las potencias de los ruidos de interferencia estimados por la metodología, se encuentran cercanos a los valores de los ruidos cardiacos 
insertados, tanto en la sección de apnea (ruidos cardiacos del uno al tres) como en la sección de respiración (ruidos cardiacos del número cuatro al doce). La observación anterior se cumple en mayor medida para la condición de $50 \mathrm{~dB}$ que para $20 \mathrm{~dB}$. Cuando la SNR para el ruido cardiaco en relación al sonido respiratorio es muy baja, por ejemplo para el quinto, séptimo, décimo y doceavo ruido insertado, la metodología sobre-estima la potencia del ruido cardiaco de interferencia e incrementa la potencia en todas las bandas de frecuencia que se analizaron. Este comportamiento se acentúa conforme el ruido de fondo se incrementa y se hace difícil detectar los ruidos cardiacos, como se muestra en la Tabla 5. 2 para el décimo ruido cardiaco insertado, en donde no se detecta la posición correspondiente y se indica un valor de cero potencia.

Los mejores resultados de estimación de la señal de ruido cardiaco de interferencia, tal y como lo indican las potencias contenidas en las Tabla 5 . 1 y 5.2, se obtienen cuando la metodología propuesta no incluye el TW. Cuando éste se utiliza, la energía de la banda de 600 a $1200 \mathrm{~Hz}$ se incrementa en relación a la potencia original del ruido cardiaco insertado. Esto debido a la observación que se estableció en la sección 6.3.

En general, en condiciones de SNR de valor alto (46 dB) a bajos (2 dB), para el ruido cardiaco insertado con relación al sonido respiratorio, sin considerar ruido de fondo, la metodología propuesta sin TW realiza un trabajo prometedor con base en la comparación de los valores de potencia entre el ruido cardiaco de interferencia insertado y el ruido cardiaco de interferencia estimado. Esta observación aún se mantiene para cuando el ruido de fondo se incrementa en el caso de $20 \mathrm{~dB}$. Sin embargo, en este caso, las bandas de $0-300$ y de 300 a $600 \mathrm{~Hz}$ son las que muestran valores más cercanos entre sí.

Con respecto al sonido respiratorio, en las tablas se incluyen las potencias del sonido respiratorio estimado, sin considerar y considerando, las potencias de los primeros IMFs, que se suponen asociados al ruido de fondo, y cuya potencia espectral dependen del nivel de ruido de fondo, 50 ó $20 \mathrm{~dB}$. Se observa que la señal de sonido respiratorio estimado se ve modificada en su potencia espectral, en comparación con 
las potencias del sonido original, principalmente en la banda de frecuencia alta, de 600 a $1200 \mathrm{~Hz}$, pero no así en la banda de frecuencia baja de 0 a $300 \mathrm{~Hz}$, en mayor medida cuando se utiliza el TW. Hay que recordar que la potencia del sonido respiratorio estimado está completamente asociado con la potencia del ruido cardiaco de interferencia estimado ya que el primero es la diferencia entre la señal primaria y la señal de salida del filtro adaptativo del esquema de eliminación de interferencias empleado (señal $y$ de la Figura 4.9).

En las tablas de 5.3 a 5.6 se presentan las potencias espectrales para un caso real para flujos de 0.5 a 2 L/seg. En estas condiciones, con propósitos de evaluar el desempeño de la metodología, se ha considerado la potencia espectral del ruido cardiaco en apnea como un estimado de la posible potencia del ruido cardiaco en respiración, es decir, del ruido de interferencia. En las tablas mencionadas, los datos más relevantes son precisamente las potencias espectrales del ruido cardiaco, en apnea y respiración, dado que la potencia original del sonido respiratorio se desconoce. En las tablas también es relevante el renglón denominado intervalos, que indica el número y las posiciones de los primeros ruidos cardiacos detectados en la zona de respiración de las señales adquiridas. En las tablas se observa, por ejemplo, que la presencia del ruido cardiaco es mayor para las señales adquiridas en la línea izquierda, Tabla 5. 3.a, b y c, que en las señales de la línea axilar derecha, Tabla 5. 3.c, d, y e. Los valores se encuentran en concordancia con la información morfológica presente en las Figura 5.10 y 5.11 , en donde se presentan las señales adquiridas a un flujo respiratorio de $0.5 \mathrm{~L} / \mathrm{seg}$. Sin embargo, la señal cardiaca es más relevante en la posición izq3, correspondiente a la porción media del tórax. Con relación a las potencias espectrales del ruido cardiaco de interferencia estimada se observa que la estimación se realiza mejor para las bandas de frecuencia de 0 a $300 \mathrm{~Hz}$ y de 300 a $600 \mathrm{~Hz}$. En la banda de frecuencia alta, de 600 a $1200 \mathrm{~Hz}$, los valores de potencia se incrementan, sobre todo si se utiliza el TW.

En las señales adquiridas en la línea axilar derecha, se observa que el ruido cardiaco de interferencia no pudo ser estimado, el renglón que indica las posiciones en que fueron detectados ruidos cardiacos de interferencia muestra valores cero en varias 
posiciones. Se puede especular, con base en el conocimiento que se obtuvo de señales simuladas, que el ruido cardiaco en estas señales presenta una SNR con respecto al sonido respiratorio baja.

En las posiciones de adquisición en donde se auscultan tradicionalmente las válvulas cardiacas, se observa una buena estimación del ruido cardiaco de interferencia, Tabla 5. 3.g a k.

En las ¡Error! No se encuentra el origen de la referencia. a la 5.54 se presenta un análisis en todos los sujetos incluidos en este trabajo del promedio de la potencia del ruido cardiaco de interferencia estimado con relación a la potencia del ruido cardiaco en apnea para las diferentes posiciones adquiridas y para los diferentes flujos considerados. Las gráficas reportan exclusivamente los resultados cuando la metodología no incluye el TW y para dos poblaciones de sujetos sanos. La nomenclatura es la siguiente: las figuras geométricas de rombos indican la potencia espectral del ruido cardiaco en apnea y los cuadrados, la potencia espectral del ruido cardiaco estimado. En ambos casos se presenta el valor promedio y la varianza. En estas figuras se observa que la potencia espectral del ruido cardiaco, tanto en promedio como en varianza, es mayor para posiciones cercanas al corazón en relación a las posiciones lejanas. Así también se observa que la estimación del ruido cardiaco de interferencia por la metodología propuesta presenta valores de potencia espectral próximos a las potencias espectrales del ruido en apnea en las bandas de 0-300 y de 300 a $600 \mathrm{~Hz}$ para todos los flujos y todas las posiciones de adquisición. En contraste, conforme el sonido respiratorio se incrementa, existe una sobre-estimación de la potencia espectral del ruido cardiaco, sobre todo en la banda de frecuencia de 600 a $1200 \mathrm{~Hz}$. Nótese también que existen valores negativos de potencia promedio en las figuras mencionadas, pero esto es solamente un efecto de restar al valor promedio la varianza obtenida.

\subsection{Conclusiones.}

El trabajo de esta tesis presenta un método alternativo de análisis con ciertas ventajas como la descomposición por EMD, que sólo depende de las características 
temporales de la señal, como el segmento de referencia a alinear que proviene de la señal adquirida, como la aplicación de una técnica de alineación para contender con posibles cambios morfológicos de la señal cardiaca. Además, se conservan las ventajas de utilizar el esquema de filtrado de interferencias utilizando el algoritmo de ajuste de pesos dado por el Least Mean Square.

El algoritmo de descomposición EMD, permite aislar eventos que son propios de la señal, de tal forma que el procesamiento se facilita, al aplicarse en los modos de oscilación de interés. La aplicación del EMD a señales acústicas respiratorias es adaptable por su naturaleza ya que depende únicamente de los datos a analizar, sin requerir la selección de algún kernel o wavelet, además con la implementación de otras herramientas como el uso de filtro adaptativo y time warping, se pretendió proporcionar una metodología más eficaz en comparación a cuado se utilizan las herramientas de manera aislada.

El esquema propuesto es bastante prometedor para la reducción del ruido cardiaco, el intento de reducción del ruido cardiaco en esta tesis, se aplicó para ell primer ruido cardiaco sin embargo la metodología es aplicable al segundo ruido cardiaco.

Por otra parte es necesario experimentar con otro tipo de algoritmo de filtrado, con la finalidad de aumentar en mayor medida la eliminación de los ruidos cardiacos con el propósito de minimizar el efecto producido por las discontinuidades de la señal alineada. Hay que considerar que existen factores que intervienen para la eliminación de los ruidos cardiacos como el nivel de ruido de fondo en la señal acústica adquirida, la amplitud de la señal acústica cardiaca, y la potencia de la señal acústica respiratoria.

Las aportaciones de esta tesis son:

1. Evaluación de la metodología en señales reales y simuladas.

2. Adquisición y prueba en 11 sujetos sanos.

3. Sistema multicanal.

4. Diferentes condiciones de flujo respiratorio.

5. Aplicación del EMD en señales acústicas provenientes del tórax. 
6. Aplicación del DTW para alineación. 


\section{Anexos}

\section{A.1 Física del Sonido.}

El término "sonido" connota dos ideas: la perturbación puramente física en un medio que separa la fuente del receptor y, el impacto psicoacústico o sensorial de esta perturbación en el cerebro del oyente. Sin embargo, en gran parte lo que oímos es el resultado de la forma en que nuestro sistema nervioso central interpreta el estímulo sonoro que recibe. De allí el término psicología del sonido y un campo de estudio relativamente nuevo con el nombre de psicoacústica.

Si bien el estudio del sonido nació con los primeros filósofos griegos, en la actualidad se basa en gran parte en las contribuciones más modernas de Von Helmholtz, Lord Rayleigh, Weber, Fechner, Flechter, Békésy y otros. La importancia del sonido en la comunicación humana se incrementó rápidamente con el desarrollo de la radiofonía y la grabación de la palabra y de la música.

\section{Producción del Sonido.}

Un cuerpo productor de sonido (fuente sonora) siempre es un cuerpo vibratorio en contacto con algún medio capaz de transmitir esta energía vibratoria al oído. Para ser escuchada la perturbación vibratoria debe tener una frecuencia comprendida dentro del orden de la audiofrecuencia o de la audibilidad, este rango va desde unos 20 ciclos o vibraciones totales por segundo, hasta un máximo de unos 20,000 ciclos por segundo. Las vibraciones inferiores a ese rango se denominan infrasónicas y aquellas cuyas frecuencias superan los 20,000 ciclos por segundo se les denomina ultrasónicas, de tal forma que las frecuencias infrasónicas y ultrasónicas no pueden percibirse como un sonido audible a pesar de que el comportamiento físico correspondiente a estos sonidos es exactamente el mismo. En forma tradicional se han clasificado las fuentes sonoras las que producen sonidos y las que producen ruido, la diferencia entre ruido y sonido, es que el sonido está compuesto por frecuencias bien localizadas, mientras que el ruido es un conjunto de sonidos mezclados y desordenados, las ondas del ruido no presentan frecuencias bien definidas, amplitud constante y su distribución es aleatoria.

\section{Transmisión y velocidad del sonido.}


La transmisión del sonido se realiza a través de un medio tangible interpuesto entre la fuente y el oído, por lo cual el vacío es un obstáculo para su transmisión o recepción. Un medio de este tipo debe de poseer dos características:

1. Debe ser elástico, lo que implica que el medio sea perturbable y tienda a recuperar su estado original.

2. Debe tener una masa o inercia que sea capaz de sobrepasar su estado de equilibrio o reposo y efectuar así un desplazamiento.

Cuanto mayor es la elasticidad y menor la densidad, más rápido se propaga el sonido a través del medio. Se puede demostrar que la velocidad de propagación del sonido en un medio dado está expresada por la relación:

$$
\text { Velocidad }=K \sqrt{\frac{\text { Elasticidad del }}{\text { Densidad del medio }}}
$$

La velocidad del sonido en el aire es de $332 \mathrm{~m} / \mathrm{seg}$ a $0^{\circ} \mathrm{C}$, lo que equivale a $1,195.2 \mathrm{Km} / \mathrm{hr}$. El incremento de la temperatura hace disminuir la densidad del aire sin que por ello afecte su elasticidad, de ahí que la velocidad del sonido en el aire caliente sea superior a la que se obtiene en el aire frío. Experimentalmente se ha encontrado, que por encima de los $0^{\circ} \mathrm{C}$ el aumento es de $60 \mathrm{~cm} / \mathrm{se}$ g por cada grado Celsius.

Es interesante hacer notar que la velocidad, la frecuencia y la intensidad del sonido en un gas, es independiente de su presión, debido a que una variación en la presión afecta en la misma proporción tanto a la elasticidad como a la densidad.

\section{Intensidad del Sonido.}

La intensidad del sonido se define como la energía que atraviesa por segundo la superficie de un volumen. Una característica importante del sonido es su energía, la energía del sonido está asociado con el desplazamiento momentáneo del medio y al movimiento de las partículas en el medio (es decir su energía cinética). Si no existen pérdidas, la suma de las dos energías permanecerá constante, sin embargo se conoce que siempre existen pérdidas expresadas normalmente en calor. La potencia acústica está relacionada con la superficie y las unidades se expresan en unidades $\mathrm{W} / \mathrm{m} 2$. La intensidad es directamente proporcional al cuadrado de la amplitud $(P)$, que es una característica fácilmente comprensible, que expresa el desplazamiento máximo de una 
partícula en el medio conductor del sonido. También, la intensidad es directamente proporcional al cuadrado de la frecuencia de la vibración.

Por otro lado, la magnitud de la sensación sonora depende de la intensidad acústica, pero también depende de la sensibilidad del oído. El intervalo de intensidades acústicas va desde el umbral de audibilidad o valor mínimo perceptible, hasta el umbral del dolor.

La intensidad fisiológica o sensación sonora de un sonido se mide en decibeles, siendo la escala logarítmica, lo que significa que el aumento de $10 \mathrm{~dB}$ corresponde a una intensidad 10 veces mayor, una intensidad de $20 \mathrm{~dB}$ representa una energía 100 veces mayor que la que corresponde a $0 \mathrm{~dB}$. La conversión entre intensidad a $\mathrm{dB}$ se expresa en la siguiente ecuación.

$$
d B=10 \log \frac{I}{I_{0}}
$$

donde $I_{0}=10^{-12} \mathrm{~W} / \mathrm{m}^{2}$ y corresponde a un nivel de 0 decibeles. En particular, el umbral del dolor corresponde a una intensidad de $1 \mathrm{~W} / \mathrm{m}^{2}$ o $120 \mathrm{~dB}$ aproximadamente.

\section{Tono.}

El tono es la característica del sonido mediante la cual el oído le asigna un lugar en la escala musical, permitiendo por lo tanto, distinguir entre los tonos graves y agudos. La magnitud física que está asociada al tono es la frecuencia. Los sonidos percibidos como graves corresponden a frecuencias bajas, mientras que los agudos son debido a frecuencias altas.

\section{Timbre.}

Cada vibración puede considerarse compleja por una serie de vibraciones armónico simples, de una frecuencia y de una amplitud determinadas, cada una de las cuales, si se considera separadamente, daría lugar a un sonido puro. Esta mezcla de tonos parciales es característica de cada instrumento y define su timbre [29].

\section{El Oído.}


El oído sano es un órgano receptor sensible al sonido, que responde a ondas de compresión y rarefacción (disminución de la densidad de un cuerpo gaseoso, como el efecto que produce un cambio de temperatura en un gas) de un amplio orden de frecuencias, intensidades y formas. Esencialmente es un sistema electro-mecánico que traduce impulsos de presión en corrientes eléctricas muy pequeñas, este fenómeno en cierto modo es muy parecido a la forma en que el cristal piezoeléctrico lo hace, donde éste sufre esfuerzos mecánicos generando una pequeña fuerza electromotriz. Las corrientes producidas en el conducto auditivo son llevadas al cerebro.

El oído externo está compuesto por el pabellón y un canal auditivo de $25 \mathrm{~mm}$ de longitud aproximadamente que canaliza las vibraciones longitudinales hacia el tímpano, el cual consiste en una membrana elástica de fuerte consistencia de aproximadamente $0.1 \mathrm{~mm}$ de espesor. El oído medio comunica las vibraciones del tímpano del oído a una serie de tres pequeños huesos: el martillo, el yunque y el estribo. Después de pasar por el oído medio, las vibraciones se dirigen a la ventana oval y finalmente, pasan al fluido coclear que llena el oído interno o laberinto. Es interesante saber que estos pequeños huesos están dispuestos de manera que crean una ganancia mecánica, es decir amplifican la señal acústica recibida.

La cóclea llena de fluido, enrollada en forma de caracol, tiene una longitud aproximadamente de $35 \mathrm{~mm}$. Está dividida en forma longitudinal en dos canales separada por la membrana basilar, donde esta última está revestida con 25,000 células pilosas o terminales de nervios del órgano de Corti. Las vibraciones del fluido coclear excitan los extremos de los nervios, haciendo que comuniquen su potencia neuronal al nervio auditivo. Los tres canales semicirculares ubicados en la parte media e interna del oído, no están involucrados en el proceso auditivo pero colaboran en cambio con nuestro sentido del equilibrio [2]. 


\section{A.2 Elementos Teóricos.}

\section{Herramientas de Procesamiento Digital Requeridas.}

Descomposición de Modos de Oscilación Empírica (EMD).

El análisis de señales es una práctica necesaria en la investigación y en aplicaciones, con dos propósitos predominantes: determinar los parámetros necesarios para la construcción de un modelo y comprobar que el modelo funciona para reconstruir los eventos que constituyen la señal. Sin embargo las señales pueden tener alguno de los siguientes problemas:

a. La señal es una muestra del evento que deseamos analizar, por lo tanto, siendo una muestra, la señal adquirida es finita.

b. Los datos pueden tener un comportamiento no estacionario.

c. Los datos pueden representar un proceso no lineal.

El análisis espectral de Fourier es un método, a través de la transformada de Fourier, que examina la distribución de la energía de la señal en el dominio de la frecuencia. Por costumbre la palabra Spectrum se ha convertido en un sinónimo de este método. Aunque la transformada de Fourier es válida en condiciones generales, tiene algunas restricciones como son:

a. El sistema debe ser lineal.

b. Los datos deben de ser estrictamente periódicos o estacionarios.

Por lo tanto, es necesario proponer otros modelos para el análisis de la señales. Entre ellos existe el análisis de señales por medio de la transformada Wavelet. La aproximación wavelet es en esencia un análisis espectral de Fourier con una ventana ajustable, y se define como sigue:

$$
W(a, b ; X, \psi)=|a|^{-1 / 2} \int_{-\infty}^{\infty} X(t) \psi^{*}\left(\frac{t-b}{a}\right) d t,
$$

donde $W(a, b ; X, \psi)$ representa la energía de la señal $\mathrm{X}(\mathrm{t})$ a la escala $a$ y al tiempo $t=b, \psi^{*}(\cdot)$ es la función wavelet básica que satisface ciertas condiciones [30]. Aunque el tiempo y la frecuencia no aparecen explícitamente en la trasformada, la variable $1 / a$ proporciona la escala de frecuencia y $b$ la localización temporal de un 
evento. Para aplicaciones específicas la función wavelet puede modificarse de acuerdo con las necesidades, pero la forma de $\psi^{*}(\cdot)$ tiene que indicarse antes de realizar el análisis. El análisis wavelet, continuo o discreto, es básicamente un análisis lineal que proporciona una resolución uniforme para todas las escalas, limitada por el tamaño de la función wavelet.

La aplicación del análisis wavelet ha sido muy popular en los últimos años por su utilidad en el análisis de frecuencia. El problema que tiene la función Morlet por ejemplo (que es la wavelet más utilizada), es la corta longitud de la función básica, lo cual hace difícil la definición cuantitativa de la distribución de la energía tiempo-frecuencia. Otro de los problemas que tiene el análisis wavelet es la localización de eventos de interés en las diferentes bandas generadas, más aún, la transformada wavelet es no adaptable, ya que la función no se define a partir de la señal que deseamos analizar.

Entre otros métodos de análisis, el Empirical Mode Descomposition (EMD) es un método adaptativo que permite analizar señales no estacionarias y no lineales; este método fue desarrollado por N. E. Huang en 1998 [22] y recientemente han surgido una serie de aplicaciones en el procesamiento de señales e imágenes biomédicas. Algunos ejemplos de señales que se han analizado con este método son:

- Señales sísmicas [31].

- Señales fisiológicas [27].

- Señales acústicas, entre otros [25].

El principio de esta técnica es descomponer cualquier señal de interés en un número finito de funciones llamadas Instrinsic Mode Function (IMF), donde la descomposición en IMFs permite la extracción directa de la energía asociada a las diferentes escalas de tiempo (las escalas de tiempo se definen como el lapso de tiempo entre dos extremos) embebidas en la señal, revelando sus características físicas como el hecho de que se conforman de múltiples eventos, en la práctica los IMFs tienen la característica de ser casi ortogonales entre ellas. 
En particular la descomposición por EMD como la descomposición por transformada wavelet genera un número finito de funciones, conservando cada IMF la misma longitud de la señal original. Los IMFs se definen cuando cumplen las siguientes condiciones:

a. El número de extremos y el número de veces que la señal cruza por cero debe de ser igual o diferir en uno.

b. El promedio de las envolventes debe ser igual a cero.

Para entender la descomposición de una señal por EMD, sea $s(t)$ cualquier señal, por lo tanto EMD representa la señal de interés por la ecuación:

$$
s(t)=\sum_{k=1}^{N} I M F_{k}(t)+r_{N}(t)
$$

donde $\mathrm{N}$ es el número de IMF's y $r_{N}(t)$ representa la señal residual. Obsérvese que si sumamos todos los IMFs obtenidos por la descomposición más el residuo, se logra reconstruir la señal original sin pérdida de información alguna, de tal forma la descomposición por el algoritmo EMD se dice que es completa.

Ahora se muestran los pasos para la descomposición en IMFs (denominado en inglés sifting process) con un ejemplo: sea $x_{1}(t)$ la suma de dos señales senoidales de diferentes frecuencias, una con una frecuencia mayor que la otra (Figura A.2.1):

1. Se identifican los extremos de la señal $x_{1}(t)$, esto es, los máximos y mínimos locales.

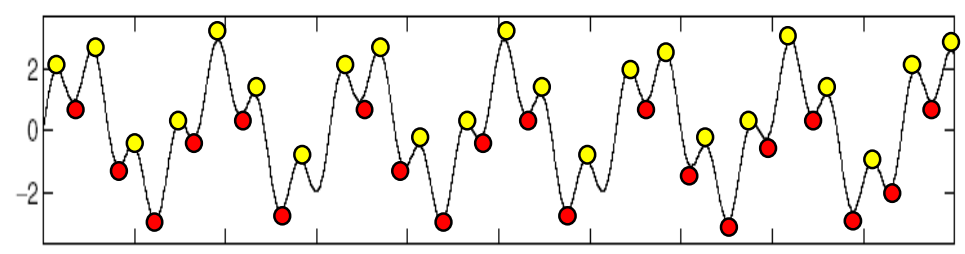

Figura A.2.1

2. Se construye una envolvente interpolando los puntos máximos y otro envolvente con los puntos mínimos localizados (Figura A.2.2).

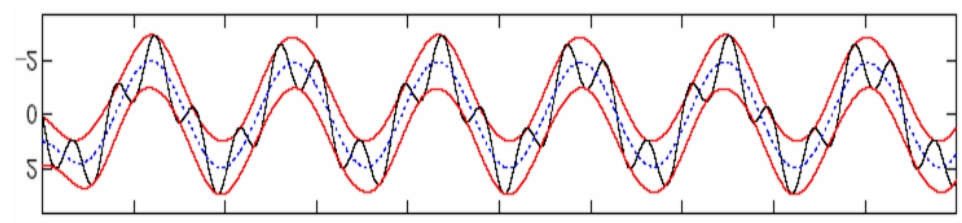

Figura A.2.2 
3. Se calcula la media entre las envolventes con la ecuación

$$
m_{1}(t)=\frac{e n v \min +e n v \max }{2}
$$

4. Se extrae el residuo $x_{2}(t)=x_{1}(t)-m_{1}(t)$.

5. Se aplica el criterio anteriormente descrito para definir una IMF (Figura A.2.3), es decir si $x_{2}(t)$ cumple con la definición de IMF, entonces se trabaja con el residuo desde el paso 1.
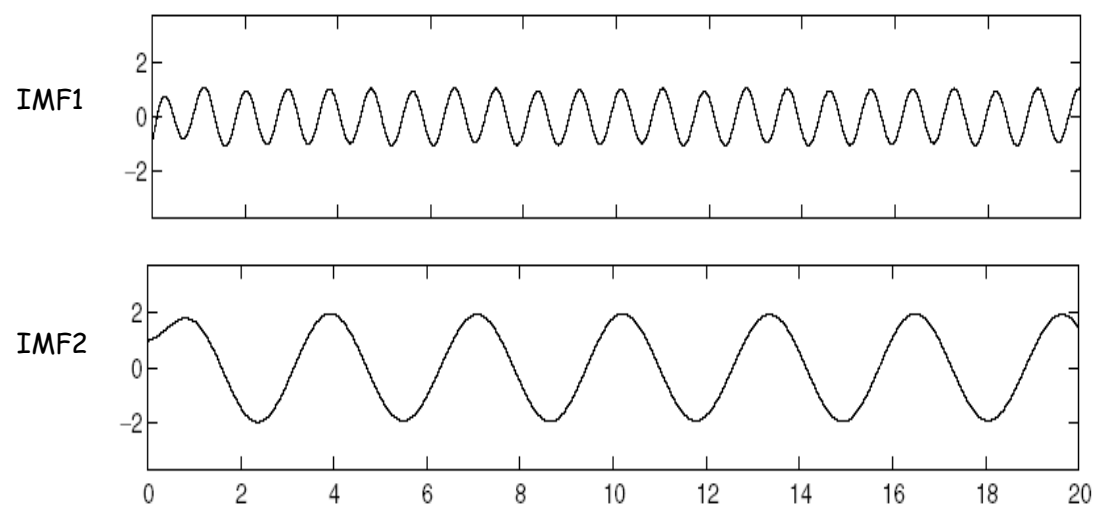

Figura A.2.3 Después de descomponer la señal inicialse obtiene dos señales similares a las iniciales.

6. En caso contrario, se continua con los mismos pasos 1 al 5 con $x_{2}(t)$. Si el procedimiento no converge a una IMF entonces se compara el residuo con un valor determinado para detener el procedimiento.

\section{La Transformada de Hilbert.}

Al igual que el espectrograma el cual utiliza la transformada de Fourier, la transformada de Hilbert nos permite analizar la frecuencia instantánea de una señal. De esta forma se podrá dar un significado físico de la energía y frecuencia ubicando diferentes eventos en el tiempo.

Dado una serie arbitraria en el tiempo $X(t)$, es posible obtener la transformada de Hilbert $Y(t)$ como:

$$
Y(t)=\frac{1}{\pi} P \int \frac{X\left(t^{\prime}\right)}{t-t^{\prime}} d t^{\prime}
$$


Donde $P$ indica los valores principales de Cauchy. Con la definición, $X(t)$ y $Y(t)$ se puede formar un par conjugado, obteniendo una señal analítica $Z(t)$ tal que:

$$
Z(t)=X(t)+\mathrm{i} Y(t)=a(t) e^{\mathrm{i} \theta(t)},
$$

donde,

$$
\begin{aligned}
& a(t)=\left[X^{2}(t)+Y^{2}(t)\right]^{\frac{1}{2}} ; \\
& \theta(t)=\arctan \frac{Y(t)}{X(t)} .
\end{aligned}
$$

Teóricamente, existen varias formas de definir la parte imaginaria, pero la transformada de Hilbert define una sola forma para obtener la función de análisis. Esencialmente la ecuación $Y(t)=\frac{1}{\pi} P \int \frac{X\left(t^{\prime}\right)}{t-t^{\prime}} d t^{\prime}$, define la transformada de Hilbert como la convolución de $X(t)$ con $1 / t$; enfatizándose las propiedades locales de $X(t)$. En la ecuación $Z(t)=X(t)+\mathrm{i} Y(t)=a(t) e^{\mathrm{i} \theta(t)}$, la expresión en coordenadas polares, clarifica en mayor medida la naturaleza local de la representación, es decir, es el mejor ajuste de la amplitud y variación de fase de la función trigonométrica para $X(t)$.

\section{Correlación Optimizada.}

Para comparar y alinear dos señales acústicas cardiacas entre sí, es decir, la señal de referencia y la de interferencia, se utilizan algoritmos de alineación. Uno de estos algoritmos es el llamado Correlation Optimized Warping que realiza una deformación lineal por segmentos de la señal, permitiendo cambios limitados en la longitud de los mismos [23]. Las señales a alinear se dividen en varios segmentos proporcionales, el objetivo es alinear un segmento muestra con un segmento de referencia. Para describir este algoritmo, consideremos dos señales, una señal de referencia $\mathrm{T}$ y una señal $\mathrm{P}$ para ser alineada, la señal resultante la llamaremos $\mathrm{P}^{\prime}$. La longitud de $\mathrm{P}$ es de $L_{p}+1$, que será dividida en intervalos con una longitud $m$ el número de secciones obtenido es: 


$$
N=\frac{L_{p}}{m}
$$

Cada sección será deformada (warped) resultando un segmento de una longitud más corta o más larga, utilizando un algoritmo de interpolación lineal para mejorar la deformación. Una sección que inicia en un punto que llamaremos $x_{s}$ y termina en un punto que llamaremos $x_{e}$ se deforma para iniciar en el punto $x_{s}{ }^{\prime} y$ terminar en el punto $x_{e}{ }^{\prime}$, calculando:

$$
p_{j}=\frac{j}{x_{e}{ }^{\prime}-x_{s}{ }^{\prime}}\left(x_{e}-x_{s}\right) ; \quad j=0, \ldots, x_{e}{ }^{\prime}-x_{s}{ }^{\prime}
$$

y posteriormente calculando el valor de $P^{\prime}\left(x_{s}{ }^{\prime}+j\right)$ obtenido vía la interpolación lineal entre los puntos de $\mathrm{P}$ y valores cercanos a $p_{j}$.

Los puntos terminales de cada sección se denominarán nodos, y la posición del punto inicial de la sección $i$, después de realizar la deformación, se llamará $x_{i}$ '. El nodo 0 es el comienzo de la primera sección $\left(x_{0}=0\right)$ y el nodo $N$ es el final de la señal completa después de la deformación. Esta posición corresponde a la longitud $L_{T}$ que corresponde a la longitud de la señal de referencia $\mathrm{T}$, por lo tanto $x_{N}=L_{T}$. De esta forma señales de diferente longitud pueden ser deformadas.

Para cada sección es posible un número finito de deformaciones. El número de deformaciones que se consideran son valores enteros de 0 a $t$, donde $t$ será referido como tensor (the slack). Es decir, t controla el incremento o decremento en la longitud del segmento. Para encontrar la mejor alineación ahora resta encontrar la combinación óptima de deformaciones en las $N$ secciones, donde cada sección es deformada no más de $t$ muestras. La deformación de la sección $i$ es llamada $u_{i}$. Dados lo valores de $t$ y $m$ es posible encontrar el intervalo de datos que se agregarán o se restarán de la sección que se va a deformar, dicho intervalo se define como $(\Delta-t, \Delta+t)$, donde $\Delta$ se define como:

$$
\Delta=\frac{L_{T}}{N}-m
$$


La calidad de la alineación se determina para cada sección $i$, calculando el coeficiente de correlación $\rho$ entre la sección $i$ después de aplicar la deformación y la sección correspondiente de la señal de referencia, es decir, $f(I)=\rho\left(I_{P^{\prime}}, I_{T}\right)$, donde $I$ denota el intervalo que se encuentra entre dos nodos. Para encontrar la combinación óptima de todas las deformaciones, es decir, la que genera la mayor suma de los coeficientes de correlación, se utiliza programación dinámica.

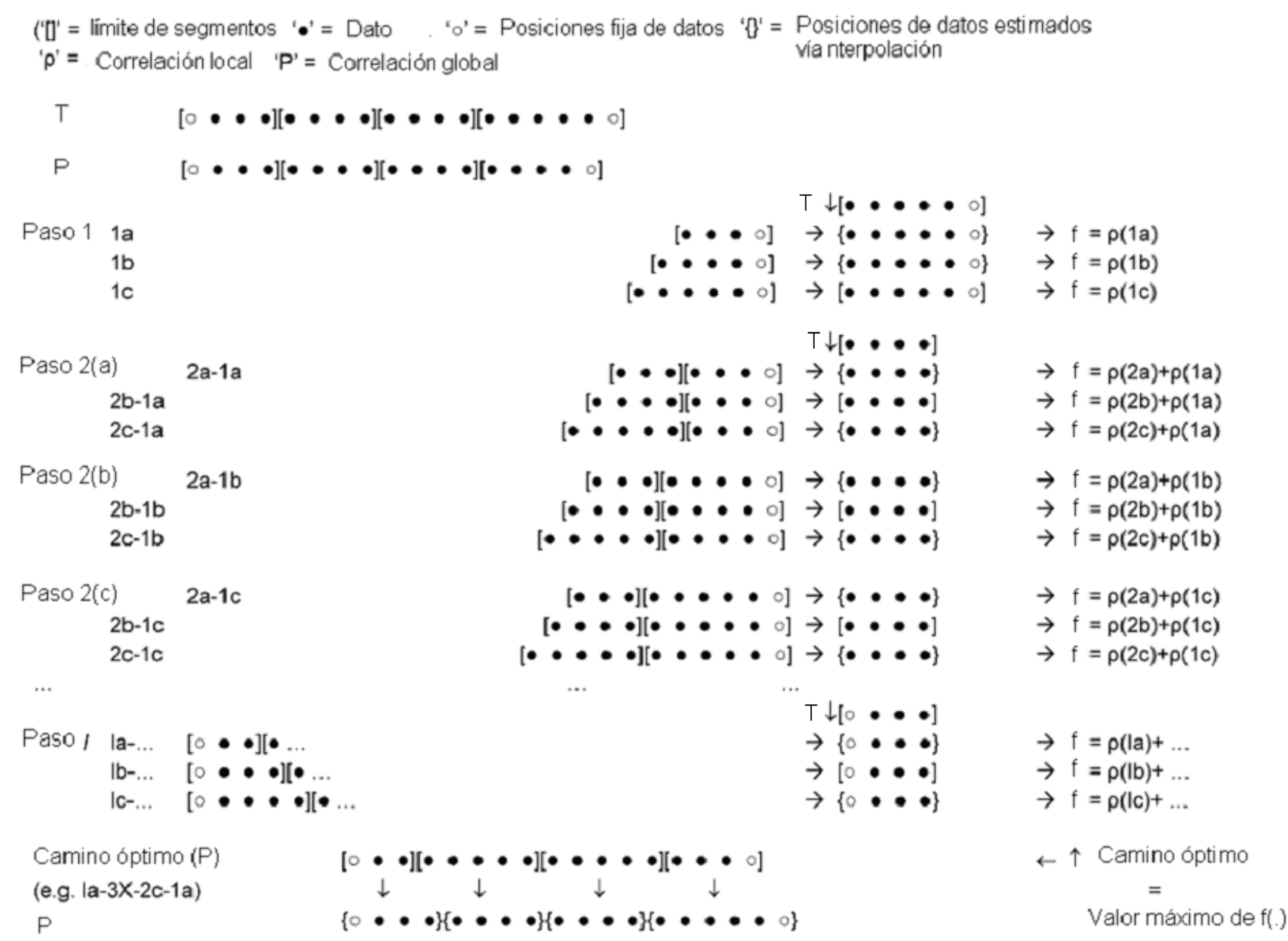

Figura A.2.4

El la Figura A.2.4 se presenta un ejemplo sencillo del procedimiento que se realiza para alinear dos secuencias de longitud de 17 y 18 muestras. Se han dividido ambas señales en $\mathrm{N}=4$ segmentos y cada segmento tiene un número $\mathrm{m}$ distinto de puntos, es decir, en el ejemplo no se consideran segmentos de igual longitud. El valor de t es 1 y por lo tanto, cada segmento puede incrementar o decrementar su longitud en un valor. En ambos casos, se requiere la interpolación lineal de los datos para lograrlo. Iniciando el procedimiento con el segmento final de la señal P hacia el segmento inicial, se tienen los siguientes pasos: 
- Con el valor de $t=1$ se tienen tres posibilidades para el último segmento de $P$ en el Paso 1, a saber el 1(a), 1(b) o 1(c).

- Se obtiene la correlación cruzada de los segmentos en 1(a), 1(b) y 1(c) con el correspondiente segmento de $\mathrm{T}$, denominada $\mathrm{f}$.

- Se toma el siguiente segmento de $\mathrm{P}$ en el paso 2. Ahora se tienen nueve combinaciones posibles entre las tres del segmento final y las tres del penúltimo segmento.

- Se calcula la correlación de cada uno de los segmentos deformados del penúltimo segmento de $\mathrm{P}$ con el segmento de referencia de $\mathrm{T}$ y el resultado se suma al valor obtenido anteriormente.

- Se continúa de esa forma y el camino óptimo para deformar cada uno de los segmentos de $P$, con $t=1$, es aquel que produce el mayor valor de la correlación total.

Sea $x^{*}$ la posición de los nodos después de la deformación bajo la combinación óptima de las deformaciones, entonces el problema queda definido como:

$$
\begin{gathered}
x_{0}=0<x_{1}<\cdots<x_{N}-1<x_{N}=L_{T} \\
u_{i} \in[\Delta-t, \Delta+t] ; \quad i=0, \ldots, N-1 \\
x_{i+1}=x_{i}+m+u_{i} ; \quad i=0, \ldots, N-1 \\
x^{*}=\arg \max x\left(\sum_{i=0}^{N-1} f\left(\left[x_{i}, x_{i+1}\right]\right)\right) \\
=\arg \max x\left(\sum_{i=0}^{N-1} \rho\left(P^{\prime}\left[x_{i}, x_{i+1}\right], T\left[x_{i}, x_{i+1}\right]\right)\right) \\
=\arg \max x\left(\sum_{i=0}^{N-1} \frac{C \operatorname{cov}\left(P^{\prime}\left[x_{i}, x_{i+1}\right], T\left[x_{i}, x_{i+1}\right]\right)}{\sqrt{V\left(P^{\prime}\left[x_{i}, x_{i}+1\right]\right) \cdot V\left(T\left[x_{i}, x_{i+1}\right]\right)}}\right)
\end{gathered}
$$

\section{Filtros Adaptativos}

La literatura ha reportado trabajos con relación a los filtros adaptativos por más de 30 años, durante este período, varios problemas teóricos se han planteado. Esta 
actividad ha sido motivada por la necesidad práctica de diseñar sofisticados sistemas para el procesamiento de señales.

Los filtros adaptativos son sistemas que poseen un finito número de parámetros que se ajustan por medio de un algoritmo que optimiza algún criterio de desempeño. Por ejemplo, el filtro adaptativo llamado beamformer, ajusta sus coeficientes para maximizar la relación señal-ruido. En un predictor adaptativo, sus coeficientes minimizan el error de predicción en sentido del error cuadrático medio. En un filtro adaptativo para la cancelación de ruido y eco, se minimiza el error cuadrático medio entre la señale de interferencia y la señal de referencia procesada por el filtro.

El procesamiento de señales utilizando filtros adaptativos, comienza con dos grandes ideas: el filtro adaptativo beamforming y el filtro adaptativo para ecualización. La revista de la IEEE "Transactions on Antenas and Propagation", publica por primera vez una sección especial de Antenas adaptables, en marzo de 1964. Los trabajos publicados en esta sección se enfocan en la retroalimentación del filtro y su automatismo de sistemas. Como ejemplo de desarrollo, en 1965, Paul Howells obtuvo una patente por el algoritmo que diseñó con el objetivo de eliminar ruido. En el mismo año en Francia, H. Mermoz publicó el resultado de su doctorado con el nombre de "Adaptive Filtering and Optimal Utilization of an Antenna", este trabajo fue fundamental para el inicio de publicaciones de filtros adaptativos en Francia. En 1967, B. Widrow, publica su tercer artículo de algoritmos adaptativos aplicado en antenas, con base en el algoritmo del error cuadrático medio (the mean square error), generando el algoritmo denominado Least Mean Square (LMS).

En 1976 el segundo especial de antenas adaptables fue publicado por la IEEE, editado por William E. Gabriel, con 15 trabajos de gran relevancia, que contribuyeron en aplicaciones de antenas adaptables. El tercer especial de algoritmos adaptativos aplicado en antenas, fue publicado en 1986, editado de igual forma por William E. Gabriel, donde se incluyeron trabajos aplicados a la estimación espectral de alta resolución.

En paralelo a los trabajos descritos, se desarrollaron otras aplicaciones de algoritmos adaptativos en el procesamiento de señales. En junio de 1981, las revistas IEEE Transactions on Circuits and Systems e IEEE Transactions on Acoustic, Speech, 
and Signal Processing publican en conjunto un especial, con trabajos con un énfasis en el algoritmo adaptativo denominado "lattice filter". Un artículo clásico es el trabajo realizados por J. Makhoul, titulado “Linear Prediction: A Tutorial Review", donde se presentan aplicaciones como la cancelación de ruido y ecos, identificación de sistemas y modelado, procesamiento de voz, estimación espectral, entre otras.

Otro trabajo clásico es el del autor Widrow, titulado "Adaptive Noise Cancelling: Principles and Aplications. Este trabajo describe el concepto de cancelación de ruido, analizando su marco teórico con aplicaciones en electrocardiografía, señales de voz, e interferencias en antenas [32].

\section{Algoritmo Adaptativo "Least Mean Square" (LMS)}

El Algoritmo adaptativo Least Mean Square (LMS), pertenece a la familia de gradiente estocástico. El término gradiente estocástico intenta distinguir al LMS del algoritmo del gradiente descendente, el cual utiliza un gradiente determinístico en el cálculo recursivo de los coeficientes del filtro de Wiener para una entrada de tipo estocástico. Una característica significativa del algoritmo LMS es su simplicidad, más aún, el algoritmo no requiere datos provenientes de la matriz de correlación de los datos de entrada y no requiere del cálculo de matrices inversas.

El algoritmo LMS es un algoritmo de filtrado adaptativo lineal, el cual contiene dos procesos básicos:

1. El proceso de filtrado involucra:

a. Cálculo de la salida del filtro lineal en respuesta de la señal de entrada.

b. Generación de error de estimación al comparar la salida del filtro con la respuesta deseada.

2. Proceso adaptativo óptimo, que involucra el ajuste automático de los parámetros del filtro acorde con el error estimado.

La combinación de estros dos procesos se llevan a cabo por medio de un lazo de retroalimentación, como se ilustra en la Figura A.2.5. En la figura las líneas gruesas indican entradas tipo vector y las líneas delgadas valores tiempo a tiempo, o muestra a muestra. 
Se cuenta con un filtro transversal o filtro RIF (la respuesta impulso o coeficientes del filtro se afectan por el LMS), el cual se encarga de realizar el proceso de filtrado de $\mathbf{u}(\mathrm{n})$. Además, el algoritmo cuenta con un mecanismo de control que se encarga de adaptar los pesos $\mathbf{w}(\mathrm{n})$ en cada paso o iteración.

Las entradas $u(n), u(n-1), \ldots, u(n-M+1)$ forman el vector de entrada $\mathbf{u}(\mathrm{n})$, donde $\mathrm{M}-1$ es el número de retrasos, que define un espacio multidimensional $\mathrm{An}$. De igual forma los pesos $\hat{w}_{0}(n), \hat{w}_{1}(n), \ldots, \hat{w}_{M}-1(n)$, forman los elementos del vector de pesos $\mathbf{W}_{0}$. El valor estimado del vector de pesos vía el estimado el LMS deberá tender a la solución de Wiener ( $\left.\mathbf{w}_{0}\right)$, conforme el número de iteraciones, $\mathrm{n}$, se aproxime al infinito.

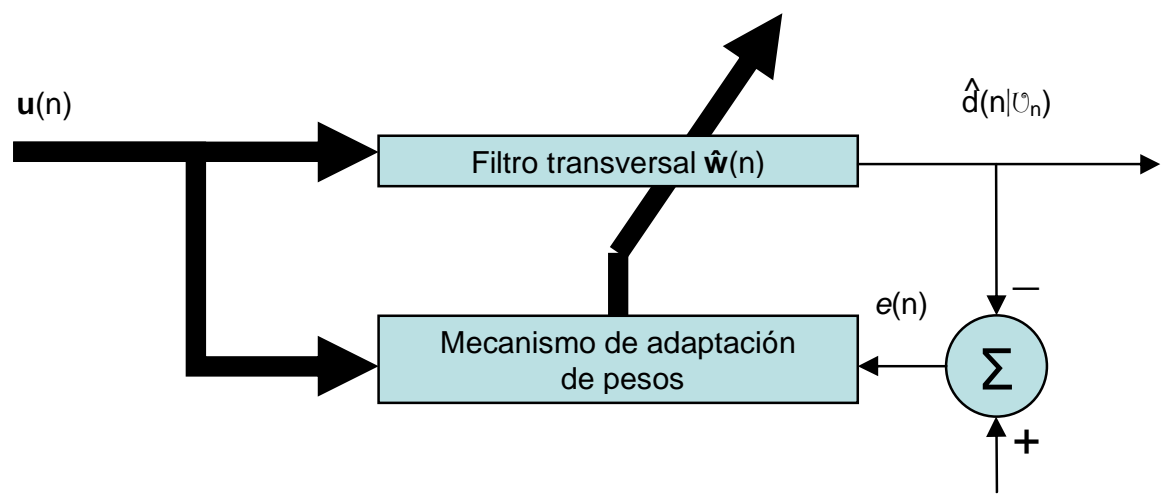

Figura A.2.5

Durante el proceso de filtrado, el vector $\mathbf{u}(n)$, produce la salida $\hat{d}\left(n \mid A_{n}\right)$ siendo ésta salida un estimado de la respuesta deseada $d(n)$. El error de estimación se puede definir como la diferencia entre la respuesta deseada y la salida actual del filtro. El error y el vector de entradas $\mathbf{u}(\mathrm{n})$ alimentan el mecanismo de control de los pesos del filtro. La Figura A.2.7 esquematiza el mecanismo de control que muestra una versión escalar del producto punto entre error estimado y la entrada $u(n-k)$ para $\mathrm{k}=0,1,2, \ldots, \mathrm{M}-2, \mathrm{M}-1$. El resultado define la corrección $\hat{\delta}_{w_{k}}(n)$ aplicado a cada uno de los pesos $\hat{w}_{k}(n)$ en la iteración $n+1$. El factor de paso utilizado en el cálculo se denota por una cantidad positiva y se le asigna la letra griega $\mu$ (step-size parameter). 


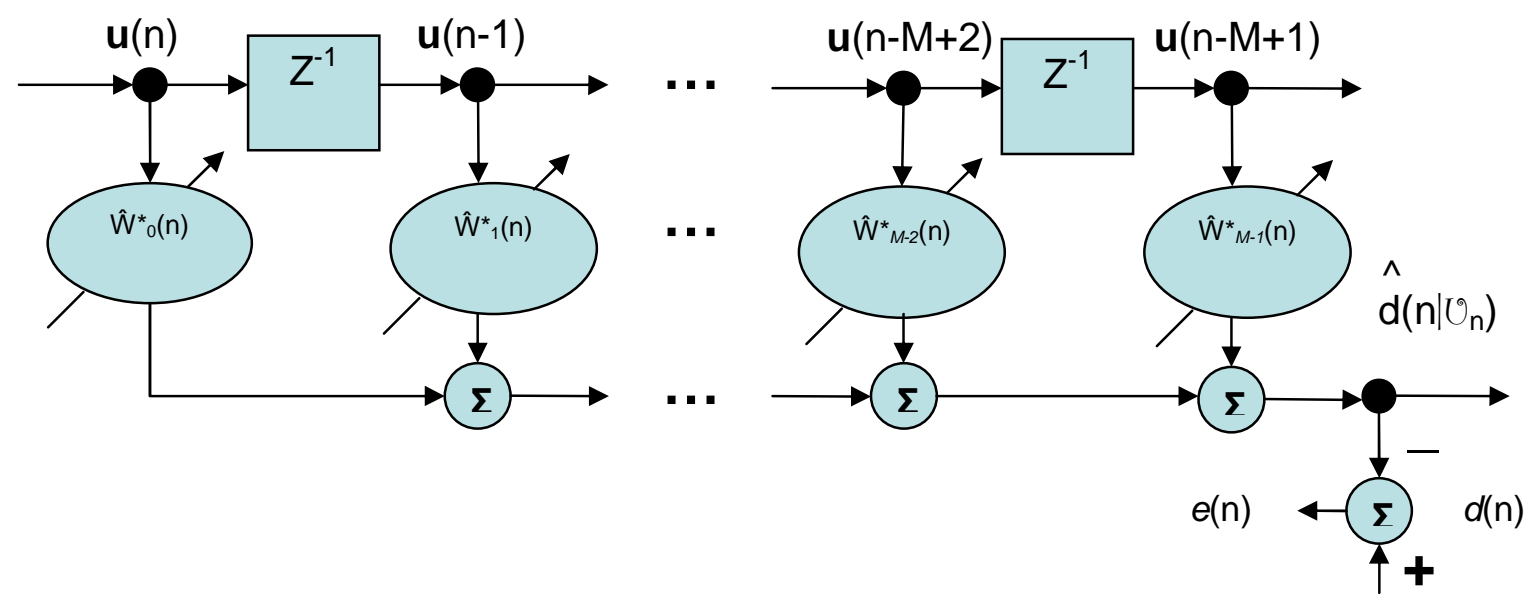

Figura A.2.6

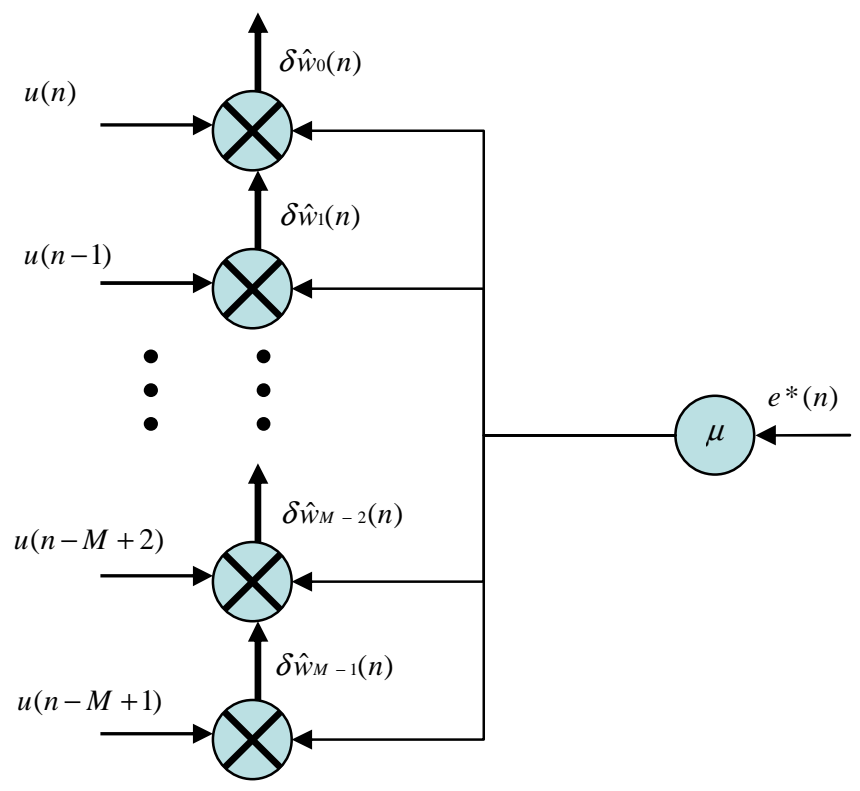

Figura A.2.7

Se asume que el vector $\mathbf{u}(\mathrm{n})$ y la respuesta deseada $d(n)$ son muestras que se obtienen de un ambiente estocástico en el sentido amplio estacionario.

El algoritmo LMS sustituye el vector gradiente necesario en el método del descenso pronunciado, para la actualización de los pesos del filtro, por una versión ruidosa obtenida directamente de los datos. 
La estrategia más obvia es sustituir los estimados de la matriz de correlación $\mathbf{R}$ y el vector de correlación cruzada p en la ecuación

$$
\nabla J(n)=-2 \mathbf{p}+2 \mathbf{R} w(n)
$$

Utilizando los estimados instantáneos para $\mathbf{R}$ y $\mathbf{p}$ :

$$
\hat{\mathbf{R}}(n)=\mathbf{u}(n) \mathbf{u}^{H}(n)
$$

Y

$$
\hat{\mathbf{p}}(n)=\mathbf{u}(n) d *(n)
$$

entonces el vector de gradiente estimado, se define:

$$
\hat{\nabla} J(n)=-2 \mathbf{u}(n) d *(n)+2 \mathbf{u}(n) \mathbf{u}(n)^{H} \hat{\mathbf{w}}(n)
$$

Obsérvese que el estimado del vector gradiente es sesgado ya que el vector de pesos estimado $\hat{\mathbf{w}}(n)$ es un vector aleatorio que depende del vector de entrada $\mathbf{u}(n)$. Sustituyendo la ecuación anterior en la ecuación del algoritmo del descenso pronunciado

$$
\begin{aligned}
e(n) & =d(n)-\hat{d}\left(n \mid U_{n}\right) \\
& =d(n)-\mathbf{w}^{H}(n) \mathbf{u}(n),
\end{aligned}
$$

se obtiene la ecuación recursiva para la actualización de los pesos del filtro:

$$
\hat{\mathbf{w}}(n+1)=\hat{\mathbf{w}}(n)+\mu \mathbf{u}(n)\left[d *(n)-\mathbf{u}^{H}(n) \hat{\mathbf{w}}(n)\right]
$$

Equivalentemente se puede escribir el resultado en tres relaciones básicas

1. Salida del filtro $y(n)=\hat{\mathbf{w}}^{H}(n) \mathbf{u}(n)$.

2. Error estimado $e(n)=d(n)-y(n)$.

3. Adaptación de los pesos $\hat{\mathbf{w}}(n+1)=\hat{\mathbf{w}}(n)+\mu \mathbf{u}(n) e^{*}(n)$.

Las ecuaciones descritas en los incisos 1 y 2 definen el error estimado, el cual esta calculado en base al vector de pesos estimado $\hat{\mathbf{w}}(n)$. Nótese que el segundo término del punto $3, \mu \mathbf{u}(n) e^{*}(n)$, representa el ajuste que es aplicado al vector estimado de los pesos $\hat{\mathbf{w}}(n)$. 
La diferencia entre el valor final del error cuadrático medio $J(n)$ obtenido por el LMS y el valor mínimo $J_{\text {min }}$, dado por la solución óptima del filtro de Wiener, es llamado error cuadrático medio de exceso $J_{e x}(\infty)$. Esta diferencia representa el precio a pagar por usar en el mecanismo de control de los pesos con base en el gradiente estimado, en lugar de utilizar una aproximación determinística, como en el método del gradiente descendente. La razón entre $J_{e x}(\infty)$ y $J_{\min }$ se llama desajuste, el cual es una medida que indica que tan lejos esta la solución del algoritmo LMS, de la solución de Wiener.

\section{Least Mean Square Normalizado.}

Los ajustes al vector de pesos en la iteración $n+1$ se realiza por medio del producto de tres términos:

- El parámetro $\mu$.

- El vector $\mathbf{u}(n)$.

- La estimación del error $e(n)$.

El ajuste es directamente proporcional al vector de entrada $\mathbf{u}(n)$. Sin embargo cuando $\mathbf{u}(n)$ es grande, el LMS sufre de un problema llamado amplificación del ruido del gradiente. Para solventar este problema es posible usar el algoritmo llamado LMS normalizado, esto quiere decir que el ajuste aplicado al vector de pesos en la iteración $n+1$ se normaliza con respecto del cuadrado de la norma euclidiana del vector de entrada $\mathbf{u}(n)$ en la iteración n-ésima.

La estructura del filtro adaptativo con base en los algoritmos LMS normalizado y el LMS estándar es exactamente la misma, ambos filtros adaptativos son construidos con base a un filtro transversal, pero difieren solamente en el mecanismo de control de los pesos. El vector de entrada $\mathbf{u}(n)$ produce una salida $y(n)$ y la diferencia entre $y(n)$ y $d(n)$ produce el error $e(n)$. En respuesta a la combinación de la entrada $\mathbf{u}(n)$ y el error $e(n)$, se modifican los pesos del filtro transversal. Esta secuencia de 
eventos se repite por un número de iteraciones hasta que el filtro alcanza cierta tendencia. Es posible modificar el algoritmo LMS original, formulando la normalización del algoritmo estándar, o es posible derivar el LMS normalizado siguiendo un procedimiento de optimización con restricciones. A continuación se detalla la segunda opción.

Para modelar el procedimiento de optimización con restricciones en términos matemáticos, denotaremos a los pesos como $\hat{\mathbf{w}}(n)$ en la iteración $n$ y con $\hat{\mathbf{w}}(n+1)$ a los pesos actualizados en la iteración $n+1$. El problema es: dado el vector de entrada $\mathbf{u}(n)$ y la respuesta deseada $d(n)$, se requiere determinar el vector $\hat{\mathbf{w}}(n+1)$ que minimice el cuadrado de la norma Euclidiana del cambio:

$$
\delta \hat{\mathbf{w}}(n+1)=\hat{\mathbf{w}}(n+1)-\hat{\mathbf{w}}(n),
$$

sujeta a la restricción:

$$
\hat{\mathbf{w}}^{H}(n+1) \mathbf{u}(n)=d(n) .
$$

Para resolver este problema de optimización con restricciones se usará el método de los multiplicadores de Lagrange. De acuerdo a este método, la función de costo consiste en dos términos como se muestra en la ecuación A.2.3.

$$
J(n)=\|\delta \hat{\mathbf{w}}(n+1)\|^{2}+\operatorname{Re}\left[\lambda *\left(d(n)-\hat{\mathbf{w}}^{H}(n+1) \mathbf{u}(n)\right)\right],
$$

donde $\lambda$ es el valor complejo de los multiplicadores de Lagrange y el asterisco denota el complejo conjugado. La norma Euclidiana cuadrática $\|\delta \hat{\mathbf{w}}(n+1)\|^{2}$ es naturalmente real. La parte real del operador denotado por $\operatorname{Re}[\bullet]$ y aplicado al segundo término, asegura que la contribución de la restricción de la función de costo es por lo tanto un valor real. La función de costo $J(n)$ es una función cuadrática en $\hat{\mathbf{w}}(n+1)$, como se muestra en la ecuación A.2.3.

$$
\begin{gathered}
J(n)=(\hat{\mathbf{w}}(n+1)-\hat{\mathbf{w}}(n))^{H}(\hat{\mathbf{w}}(n+1)-\hat{\mathbf{w}}(n)+\ldots \\
\ldots+\operatorname{Re}[\lambda(d(n)-\hat{\mathbf{w}}(n+1) \mathbf{u}(n))]
\end{gathered}
$$


Para encontrar el valor óptimo de los pesos actualizados que minimicen la función de costo $J(n)$, se realizan los siguientes pasos:

1. Diferenciar la función de costo con respecto a $\hat{\mathbf{w}}(n+1)$, entonces $\frac{\partial J(n)}{\partial \hat{\mathbf{w}}(n+1)}=2(\hat{\mathbf{w}}(n+1)-\hat{\mathbf{w}}(n))-\lambda * \mathbf{u}(n)$. Igualando este resultado a cero obtenemos la siguiente ecuación $\hat{\mathbf{w}}(n+1)=\hat{\mathbf{w}}(n)+\frac{1}{2} \lambda * \mathbf{u}(n)$.

2. Resolver el multiplicador no conocido $\lambda$ sustituyendo el resultado del paso 1

$$
\begin{aligned}
d(n) & =\hat{\mathbf{w}}^{H}(n+1) \mathbf{u}(n) \\
& =\left(\hat{\mathbf{w}}(n)+\frac{1}{2} \lambda * \mathbf{u}(n)\right)^{H} \mathbf{u}(n) \\
& =\hat{\mathbf{w}}^{H}(n) \mathbf{u}(n)+\frac{1}{2} \lambda \mathbf{u}^{H}(n) \mathbf{u}(n) \\
& =\hat{\mathbf{w}}^{H}(n) \mathbf{u}(n)+\frac{1}{2} \lambda\|\mathbf{u}(n)\|^{2} .
\end{aligned}
$$

Resolviendo para $\lambda$ obtenemos

$$
\lambda=\frac{2 e(n)}{\|\mathbf{u}(n)\|^{2}}
$$

Donde

$$
e(n)=d(n)-\mathbf{w}^{H}(n) \mathbf{u}(n)
$$

3. Combina los resultados del paso $1 \mathrm{y}$ el paso 2 para formular el valor óptimo del incremento $\delta \hat{\mathbf{w}}(n+1)$. Específicamente de las ecuación A.2.5 y A.2.6, tenemos

$$
\begin{aligned}
\delta \hat{\mathbf{w}}(n+1) & =\hat{\mathbf{w}}(n+1)-\hat{\mathbf{w}}(n) \\
& =\frac{1}{\|\mathbf{u}(n)\|^{2}} \mathbf{u}(n) e^{*}(n) .
\end{aligned}
$$

Para controlar el cambio del vector de peso de una iteración a otra, sin cambiar la dirección del vector, introducimos un factor real positivo denotado como $\tilde{\mu}$. Esto es, redefiniendo el cambio como: 


$$
\begin{aligned}
\delta \hat{\mathbf{w}}(n+1) & =\hat{\mathbf{w}}(n+1)-\hat{\mathbf{w}}(n) \\
& =\frac{\tilde{\mu}}{\|\mathbf{u}(n)\|^{2}} \mathbf{u}(n) e^{*}(n) .
\end{aligned}
$$

Equivalentemente se puede escribir:

$$
\hat{\mathbf{w}}(n+1)=\hat{\mathbf{w}}(n)+\frac{\tilde{\mu}}{\|\mathbf{u}(n)\|^{2}} \mathbf{u}(n) e^{*}(n)
$$

de esta última ecuación se obtiene la recursión para calcular el vector de pesos en el algoritmo LMS normalizado. Claramente se muestra la razón del término normalizado, ya que el producto $\mathbf{u}(n) e^{*}(n)$ es normalizado con respecto al cuadrado de la norma Euclidiana del vector de entrada $\mathbf{u}(n)$.

Comparando la recursión de la ecuación A.2.8 del algoritmo LMS normalizado con la ecuación que pertenece al algoritmo LMS convencional, es posible hacer la siguiente observación:

- De la ecuación $\mu(n)=\frac{\tilde{\mu}}{\|\mathbf{u}(n)\|^{2}}$, se puede ver que la normalización del algoritmo LMS convierte al algoritmo LMS convencional en uno con un parámetro de paso variable con el tiempo [24]. 
Referencias.

[1] H. C. Hinshaw, Enfermedades del Tórax, McGrawHill, pp. 133-154, 1983.

[2] J. R. Escajadillo, Oídos, Nariz, Garganta y Cirugía de Cabeza y Cuello, Manual Moderno, pp. 415-490, 2002.

[3] H. Pasterkamp, S. S. Kraman, G. Wodicka, "Respiratory sounds advances beyond the stethoscope," Am. J. Respir. Crit. Care Med., vol 156, pp. 974-987, 1997.

[4] A. C. Guyton, Tratado de Fisiología Médica, McGrawHill, pp. 115-173, 1997.

[5] R. G. Loudon, "Lung sounds," Current Pulmonary, vol. 9, cap. 3, 1988.

[6] M. D. Zalter, "Phonocardiography", Clinical Cardiopulmonary Physiology, American Collage of Chest Physicians, cap. 6, 1990.

[7] M. J. Mussell, "The need for standards in recording and analysis of respiratory sounds, ” Med. Biol. Eng. Comput., vol. 30, pp. 129-139, 1992.

[8] J. C. Wood, D. T. Barry, "Quantification of first Heart sound frecuency dynamics across the human chest wall," Med. Biol. Eng. and Comput. vol. 32, pp. S71-S78, 1994.

[9] W. Hui Winnie, R. A. Pitt, J. P. Matonik, J.K-J. Li, "Comparison of heart sounds recorded at the chest and a remote arterial site," Proc. Bioeng. Conf., IEEE $28^{\text {th }}$ Ann. Northeast Volume, Issue, pp. 61 - 62, 2002.

[10] B. Tovar-Corona, "Effects of respiration on heart sounds using time-frequency analysis," Computers in Cardiology, IEEE, pp. 457-460, 2001.

[11] I. Hossain, Z. Moussavi, "Finding the lung-sound flow relationship in normal and asthmatic subjects," Proc. 26th Ann. Int. Conf. IEEE EMBS, pp. 3852-3855, 2004.

[12] N. Gavriely, D. W. Cugell, "Airflow effects on amplitude and spectral content of normal breath sounds," J. Appl. Physiol., vol. 80, pp. 5-13, 1996.

[13] R. González, S. Carrasco, S. Charleston, J. Gaitán, G. Ramos, G. Chi, R, Pérez, "Sistema para el análisis cuantitativo de los sonidos respiratorios", Rev. Mex. Ing. Biom., vol. 17, num. 2, pp. 7-23, 1997.

[14] V.A. Mackusick, J.T. Jenkins, G.N. Webb, "The acoustic basis of the examination", Am. Rev. Tuberc., vol. 72, pp. 12-34, 1953.

[15] J.E. Earis, B.M.G. Cheetham, "Future perspectives for respiratory sound research," Eur. Respir. Rev., vol. 10, no 77, pp 641-646, 2000. 
[16] A. R. A. Sovijärvi, J. Vandersschoot, J. E. Earis, "Standardization of computerized respiratory sound analysis," Eur. Respir. Rev., vol. 10, pp. 585, 2000.

[17] S. Charleston, S. Cortés. R. González, G. Chi, T. Aljama "Respiratory acoustic thoracic imaging (RATHI): assessing deterministic interpolation techniques," Med. Biol. Eng. Comput., vol.42, pp. 618-626, 2004.

[18] V. K. lyer, P. A. Ramamoorthy, H. Fan, and Y. Ploysongsang, "Reduction of heart sounds from lung sounds by adaptive filtering", IEEE T. Biomed. Eng., vol. 33, no. 12, pp. 1141-1148, 1986.

[19] J. Gnitecki, Z. Moussavi and H. Pasterkamp, "Recursive least squares adaptive noise cancellation filtering for heart sound reduction in lung sounds recordings," Proc. $25^{\text {th }}$ Ann. Int. Conf. IEEE-EMBC, pp. 2416-2419, 2003.

[20] S. Charleston, and M. R. Azimi-Sadjadi, "Reduced order Kalman filtering for the enhancement of respiratory sounds," IEEE Trans. Biomed. Eng., vol. 43, no. 4, pp. 421-424, April 1996.

[21] S. Charleston, M. R. Azimi-Sadjadi, and R. González, "Interference cancellation in respiratory sounds via a multiresolution joint time-delay and signal-estimation scheme”, IEEE Trans. Biomed. Eng., vol. 44, no. 10, pp. 1006-1019, October 1997.

[22] G. Rilling, "On empirical mode decomposition and its algorithms", http://perso.enslyon.fr/paulo.goncalves/pub/emd-eurasip03.pdf.

[23] G. Tomasi, F. van den Berg, C. Andersson, "Correlation optimized warping and dynamic time warping as preprocessing methods for chromatographic data", J. Chemometrics, no. 18, pp. 231-241, 2004.

[24] S. Haykin, Adaptive Filters Theory, Prentice Hall, 4ª edition, 2002.

[25] S. Charleston, L. F. Domínguez, R. González, T. Aljama, "Heart sounds interference cancellation by empirical mode decomposition for lung sounds analysis," Proc. 28 ${ }^{\text {th }}$ Ann. Int. Conf. IEEE-EMBS, pp. 1694-1697, 2006.

[26] H. G. Martínez-Hernández, A. T. Aljama-Corrales, R. González-Camarena, S. Charleston-Villalobos, G. Chi-Lem, "Computerized classification of normal and abnormal lung sounds by multivariate linear autoregressive model," Proc. $27^{\text {th }}$ Ann. Conf. IEEE-EMBS, pp. 1464-1467, 2005. 
[27] H. Liang, Z. Lin, R. W. McCallum, "Artifact reduction in electrogastrogram based on empirical mode descomposition method," Med. Biol. Eng. Comput., vol. 38, pp. 3541, 2000.

[28] R. Balocchi, D. Menicucci, E. Santarcangelo, L. Sebastiani, A. Gemignani, B. Ghelarducci, M. Varanini, "Deriving the respiratory sinus arrhythmia from the heartbeat time series using Empirical Mode Descomposition," Chaos, Solitions \& Fractals, vol. 20, Issue 1, pp. 171-177, April 2004.

[29] A. Efron, El Mundo del Sonido, Marcombo, pp. 1-123, 1990.

[30] N. E. Huang, "The empirical mode decomposition and the Hilbert spectrum for nonlinear and non-stationary times series analysis", Mathematical, Physical and Engineering Sciences, vol. 454, no. 1971, pp. 903-995, 1996.

[31] I. Magrin-Chagnolleau, "Empirical mode decomposition based time frequency attributes", http://cmc.rice.edu/docs/docs/Mag1999Non5Empirical.ps.

[32] L. H. Sibulb, Adaptive Signal Processing, IEEE PRESS, pp. 3-4, 149-151, 1987. 
Tabla de Figuras 
